\title{
Age-related cognitive decline and cognition enhancers
}

Citation for published version (APA):

Hogervorst, E. (1998). Age-related cognitive decline and cognition enhancers. [Doctoral Thesis, Maastricht University]. NeuroPsych Publishers. https://doi.org/10.26481/dis.19980402eh

Document status and date:

Published: 01/01/1998

DOI:

10.26481/dis.19980402eh

Document Version:

Publisher's PDF, also known as Version of record

\section{Please check the document version of this publication:}

- A submitted manuscript is the version of the article upon submission and before peer-review. There can be important differences between the submitted version and the official published version of record.

People interested in the research are advised to contact the author for the final version of the publication, or visit the DOI to the publisher's website.

- The final author version and the galley proof are versions of the publication after peer review.

- The final published version features the final layout of the paper including the volume, issue and page numbers.

Link to publication

\footnotetext{
General rights rights.

- You may freely distribute the URL identifying the publication in the public portal. please follow below link for the End User Agreement:

www.umlib.nl/taverne-license

Take down policy

If you believe that this document breaches copyright please contact us at:

repository@maastrichtuniversity.nl

providing details and we will investigate your claim.
}

Copyright and moral rights for the publications made accessible in the public portal are retained by the authors and/or other copyright owners and it is a condition of accessing publications that users recognise and abide by the legal requirements associated with these

- Users may download and print one copy of any publication from the public portal for the purpose of private study or research.

- You may not further distribute the material or use it for any profit-making activity or commercial gain

If the publication is distributed under the terms of Article $25 \mathrm{fa}$ of the Dutch Copyright Act, indicated by the "Taverne" license above, 


\title{
Age-related Cognitive Decline and
}

\author{
Cognition Enhancers
}




\section{Neuropsych Publishers Mastricht}

Production: Datawyse I Uniwersity Press Maastricht

E. Hogervorsi, Maastricht 1998

Age related cognitiwe decline and cognition enhancers / Eef Hogervorst. - Maastricht:

Neuropsych Publishers Maastricht. - 111.

ISBN 90-75579-06-3

NUGI 716731743

Neuropsych Publishers is a non-profit organisation which aims at promoting the science of "Brain \& Behavior" and imjoroving the applicition of the products of this science in health care and education. Neuropsych Publishers accomplishes these aims by publishing books, dissertations and other products of scientifie activity, by disemminating educational materials and publication of tests, assessment scales and other psychometric instruments in the field of Neuropsychology, Neuropsychiatry, and other areas within the dontain of Brain \& Behavior.

Postal address:

Neuropsych Publishers

Department of Psychiatry \& Neuropsychology

Section Neuropsychology< Neuropsychiatry, and Psychobiology

Universiteil Maastricht

POBOx 616

NL-6200 MD Maasurich

The Netherlands

This publication has been supported by grants of the Isostar Sport Nutrition Foundation: Bern, Switzerland. The author also wishes to thank Bayer B.V., Hoechst Marion Roussel B.V., Novartis Pharma B.V, Novo Nordisk Farma B.V., Organon International N.V., Servier R\&D Benelux, and UCB Pharma B.V. for their financiall support of the publication of this thesis. 


\section{Age-Related Cognitive Decline And \\ Cognition Enhancers}

\section{PROEFSCHRIFT}

ter verkrijging van de graad van doctor aan de Universiteit Maastricht op gezag van Prof. dr. A. C. Nieuwenhuijzen Kruseman, Rector Magnificus volgens het besluit wan het College van Decanen,

in het openbaar te verdedigen op

donderdag 2 April 1998 om 16:00 uur

door

Eva Hogervorst

geboren op 16 juli 1968 te Heerlen 
Promotor:

Co-promotor:

Beoordeling cominissie:
Prof. dr. I. Jolles

Di. W. I. Riedel

Prof. ar. H. L. G. J. Merckelbach (voonzitter)

Prof. dr. A. W. K. Gaillard (Kaholieke Universiteit Brabant)

Prof. dr. E. J. L Griez

Prof. dr. W. H. M. Saris

Prof. dr. K. Wesnes (Cognitive Drug Research Ltd, Reading, U.K.)

The studies described in this thesis were carried out at the Maastricht Brain \& Behavior Institute, the Department of Psychiatry \& Neuropsychology of Maastricht University, and the University Hospital Maastricht, the Netherlands. 
Ik draag dit proefschrift op aan mijn vader Frans Hogervorst ondat hij altijd een wrije lefgozer is gebleven zonder zijn gevoel voor humor te verliezen 


\section{Chapter 1}

\section{Introduction}

\subsection{Age-related cognitive decline}

There has been a steady increase in the proportion elderly in the population since the beginning of this century. In the year $2000,13.6 \%$ of the people in the Netherlands will be over 65 years of age. This percentage will have almost doubled in 2040 (CBS, 1997). The majority of elderly people complains of forgetfulness and decreased concentration (Jolles, Houx, Van Boxtel, \& Ponds, 1995). In addition, decreased cognitive functions can be objectified in the elderly, as it is well established that virtually all aspects of cognitive function deteriorate with age (Birren \& Schaie, 1990; Houx \& Jolles, 1993; Huppert, 1991; Kok, Looren do Jong, \& Van Rooy, 1988; Salmaso, 1993; Verhaeghen, Marcoen, \& Goossens, 1993). Not all individuals show the same rate of decline in cognitive functioning. There is a wide range from "successful cognitive aging" (Rowe \& Kahn, 1987) to pathological conditions, such as Alzheimer's Disease. Thus, age-associated cognitive changes vary across a continum. Successful aging is defined by a cognitive performance which is above average as compared to age peers, but within the normal range for younger adults (Rediess \& Caine, 1996). The borderline conditions between normal to successful cognitive aging and dementia consist of people with objective cognitive impairment as compared to aged peers. In patients suffering from dementia, the functional changes are much more widespread, as is seen in the gradual decrement of memory processes, planning and many other cognitive processes as well as emotional and social behaviors (Jolles, Verhey, Riedel \& Houx, 1996). Thus, a large variability in cognitive performance exists among aging subjects.

The quest for a high quality of life in the autumn years has created a market for the medical treatment of cognitive deficiency. Of the subjects that reported themselves as being forgetful in a large population studly, about a quarter was interested in treatment with medication (Commissaris, Ponds, \& Jolles, 1995). A wide range of potential cognition enhancers has been developed over the years. A cognition enhancer is by definition a drug that restores impaired cognitive functioning (Gamzu, Birkhimer, Hoover, \& Gracon, 1990). A cognition enhancer can act by counteracting factors which are thought to play a role in aging, such as the neurotransmitter deficiencies, brain hypoxia due to atherosclerotic processes, and/or the cell damaging effects of oxidative stress (Spiegel, 1995). Yet, as Coper and Kanowski (1983) stated, specific uniform mechanism of action of cognition enhancers often is not known.

One of the main problens in this area of research has been that the success rate for detecting useful drugs is poor (Hollister, 1990). This may have been caused by the following factors. First, in the research into cognition enhancers there is a lack of a valid standard index for cognitive aging. Some of the tests used may lack sensitiwity to detect mild changes (Bergener, 1993), Second, as mentioned, in the aged a large variation occurs in cognitive functioning. Thus, a large sample is needed and in some studies the power may not have been adequate to show significant effects. Third, no drug has been fully accepted to be effective enough. Hence, no drug is available against which to compare other drugs for predictive value, the so called "golden standard" (Hollister, 1990). Fourth, and finally, there is a lack of well-evalluated models of cognitive aging to use in preclimical studies with putative cognition enhancing dirugs. 


\subsection{Models and treatment of age-related cognitive decline}

A major problem in research into cognition enhancers is thus concerned with the method used to allow an early evaluation of the possible efficacy of drugs. Models of cognitive dysfunction have been deweloped for this purpose. The major premises are the following. First, the model should temporarily induce a cognitive deficiency. Then, a potential drug or cognition enhancer should reverse this cognitive deficiency. As such, a model can provide insight in both the pathogenesis atnd the treatment of pathological aging. Scopolamine is a cholinergic antagonist and models the cholinergic deficiency thought to be implicated in some of the cognitive dysfunctions seen in aging and dementia. The scopolamine model has been widely used in the past; but it has received criticism, e.g. with respect to its specificity. Suggestions have been done to improve existing models and to develop new models. In recent years, the hypoxia model, and an alternative, the hyperventilation model, and various others have been proposed and tested in preclinical experiments. However, most models have not been evaluated adequately.

The present thesis attempts to address this problem and contains articles which describe experimental research into the possible usefulness of various models for age-related cognitive decline. The aim was to evaluate the possible effectiveness of models of age-related decline in healthy subjects. The rationale was that testing possible cognition enhancers in healthy young, but also middle-aged subjects may have advantages over clinical experiments. This method is less expensive and time consuming since due to controlled testing of homogenous groups, less subjects are needed. In addition, there may be practical and ethical advantages in the use of healthy volunteers for drug-evaluation studies.

Recentlly, Riedel et al (1995) found that caffeine was able to counteract the scopolamine-induced memory impairment in healthy subjects and suggested that caffeine may have the potency to act as such a golden standard. The present thesis aims at evaluating potential models of age-related decline and testing cognitive effects of the most ubiquitously used drug in the world: caffeine. Caffeine as a possible 'golden standard drug' is used in several of the articles.

Furthermore, there is a growing interest in cognitive side-effects of hormones, such as estrogens. which suggests that Hormonal Replacement Therapy (HRT) may counteract the cognitive deficiency in middle-aged and elderly women, and may even delay the onset of Alzheimer"s Disease. However, experimental flaws render evaluation of this treatment difficult. This thesis also attempts to critically evaluate the merits of clinical HRT. The next paragraph describes the aims for this thesis in more detail.

\subsection{Aims and outline of this thesis}

This thesis is concerned with age-related cognitive deficiencies and the evaluation of possible relevant methods to investigate the effect of putative cognition enhancers. The thesis is divided in three parts. Part one describes experimental studies into models of age-related cognitive deficiency. Part two describes studies into the effect of caffeine in models of cognitive deficiency. Part three addresses the effects of HRT on age-related cognitive deficiency as an example of the clinical approach in testing cognition enhancers in nondemented elderly.

\section{Part one-Models of age-related cognitive deficiency}

In part one, chapter three and four describe the age-related performance on a conplex memory test. This new memory test was compared to a standard memory test in a large population sample (chapter three). In this study also the possible intermediate effects of the age-related decrease in 
cognitive slowing and health on memory functions was inwestigated: A second study is deseribed in chapter four which included only healthy subjects of three age-groups, and which assessed the relative contribution of the slowing of sensorimotor functions and working memory on the performance of the complex memory test. This study was illustrative for the fact that large homogenous groups of elderly subjects are not easily found. As such, this research method is time consuming and expensive. The homogeneity in groups of young adult volunteers (e.g. students) creates an attractive alternative.

In using healthy, optimally functioning subjects, a state of diminished fonctioning has to be induced to model the cognitive dysfunctions seen in aging. A model of agerelated cognitive deficiency can be used to give an indication of the range and duration of the therapeutic effect of a potential cognition enhancer on cognitive dysfunction. Also, a model can give insight in the factors that contribute to diminished mental functioning, such as brain hypoxia or neurotransmitter deficiencies. There are several methods to induce a cognitive dysfunction in young subjects. In part one, chapters two, five, and six the effect of several models on the cognitive profile in the age-sensitive cognitive test battery, their validity with respect to factors thought to be important in aging, and their usefulness in testing cognition enhancers is described.

\section{Part two - The effects of caffeine on age-related cognitive deficiency"}

Although piracetam analogues are often used for this purpose, an adequate "golden standard drug' with undisputed cognition enhaneing properties has not yet been found. Recently, caffeine has clearly been seen to improve cognitive functions. Interestingly, this effect was especially apparent in elderly subjects (Jarvis, 1993). In fact, elderly may be more sensitive to the central effects of caffeine (Swift \& Tiplady, 1988). Caffeine, in coffee and tea, is consumed daily by hundreds of millions of people all over the world and has relatively little side effects (Klatsky, Armstrong, \& Friedman, 1993). Further, Riedel et al (1995) found that caffeine could act as a cognition enhancer in the scopolamine (a cholinergic antagonist) model. They proposed that caffeine could be used as a control drug and that effects of a newly developed cognition enhancer should be at least superior to the effect of 3 cups of coffee. In part two, chapters seven, eight, and nine, effects of caffeine in models of age-related cognitive dysfunction are described.

\section{Pant three - The effects of gonadal hormone suppletion therapy on age-related cognitive deficiency}

Lastly, some authors debate whether the results obtained with young healthy subjects can be extrapolated to elderly (mostly ill) subjects and conclude that studies with normal young wolunteers cannot be indicative of a therapeutic effect, since the difference in the bodily response may affect the response to drugs (Gamzu, Birkhimer, Hoover, \& Gracon, 1990). Indeed, a model of cognitive dysfunction in young humans cannot mimic the effects of time, and the interactions of biological factors with time. For some drugs which act through their antioxidant properties in the brain or by conserving physiological systems over at larger period of time, this may be true. Gonadal hormones, such as estrogens, have been reported in both epidemiological and experimental studies to delay the age of onset in Azheimer's disease (Tang et al., 1996), to have a protective effect in developing vascular dementia (Lopes, Mensier, Laurent, \& Besse, 1995), and to enhance cognitive functioning in postmenopausal women (Sherwin, 1994). However, many methodological issues hamper clear cut conclusions. Part three of thiss thesis is concerned with both short and long term effects of estrogens on cognitive functioning in elderly normal and demented women. In chapter ten, methodological issues are discussed in at review. In chapter eleven, effects of the use of Hormonal Replacement Therapy (HRT) in a large population sample 
are described, followed by the results of a follow-up into the effects of HRT on cognitive functioning in normal postmenopausal dutch women in chapter twelve. A general discussion of the usefulness - and future perspectives of research into - models of age-related cognitive dysfunction and cogmition enhancers will conclude this thesis.

\subsection{Questionis addressed in this theses}

1. Which cognitive tests should be used in order to measure the effect of age-related cognitive decline and the effect of cognition enhancers?

In chapter three and four, two studies are presented in which a complex age-sensitive memory test was tested. One study deseribes the comparison of performance of the new memory test with the standard memory test in a large population sample. A second study was perfomed, which included only healthy subjects and which assessed the relative contribution of the slowing of sensorimotor functions and working memory on complex memory performance.

2. Which models can be used to mimic age-related cognitive decline and to test potential cognition enhancers?

Part one describes several models of age-related cognitive decline. First, an evaluation is given of several models of cognitive dysfunction in chapter two followed by a study of the hypoxia and hyperventilation model and a study of maximal physical exhaustion (chapters five and six)

3. Is caffeine a valid "golden standard" drug to evaluate the effect of a potential cognition enhancer in models of age-related cognitive decline?

The effects of caffeine on cognitive performance in three separate age-groups is discussed in chapter seven. The effects of caffeine in two models of age-related cognitive dysfunction is discussed in chapters eight and nine.

4. What is the nature of the effect of Hormonal Replacement Therapy on age-related cognitive decline in natural postmenopausal women?

A rewiew is presented in chapter ten, in which the effects of Hormonal Replacement Therapy (HRT) in elderly and demented women are critically discussed. Chapter eleven concerns a case-control study which investigated the effects of HRT on cognitive functions in a population sample. In chapter twelve, a study into the long term effects of HRT on cognitive functions in postmenopausal women is presented. 


\section{PART I : MODELS OF COGNITIVE DYSFUNCTION}

\section{Chapter 2}

\section{Models of age-related cognitive deficiency and dementia}

\subsection{Introduction}

There is an age-related decline in nearly all cognitive functions, especially the performance on tasks of memory, problem solving, and perception decreases with age. Likewise, the speed and efficiency at which information processing tasks are carried out compromises with increasing age (Birren \& Schaie, 1990; Jolles, Verhey, Riedel, \& Houx, 1995b). Not all aged people are characterized by the same deterioration. There is a large variability in cognitive performance within people of the same age. The increase in the variability in cognitive functioning of elderly subjects is known to be caused in some part by inter.individual differences in the level of eduction or job requirements (Jolles, Houx, Van Boxtel, \& Ponds, 1995a). Also, the difference in health status may contribute to the variation seen (Salmaso, 1993). More in general, it has been hypothesized that adverse biological life events, such as the long term use of benzodiazepines and alcohol or the number of headtraumas, could largely explain the variability in cognitive functioning (Houx \& Jolles, 1994; Houx, Vreeling, \& Jolles, 1991). However, the impact of biological factors on mental functioning in the course of aging and in the eventual development of dementia still remains largely unclear.

The aging of the society is accompanied by a strong pressure to develop drugs that treat the cognitive decline that can accompany aging and the pathological conditions, such as dementia (Pepeu, 1994). Yel, no drug has been reliably demonstrated to be efficacious in the treatment of Age-Associated Cognitive Decline (AACD) (Riedel \& Jolles, 1996). Recently, some drugs (e.g. tacrine, donepezil) have been developed which seem to retard the process of deterioration of Alzheimer"s' Dementia to a modest extent (Bryson \& Benfield, 1997). Experimental drug reseatrh in non demented subjects up to now has been less popular than drug research in patients with Alzheimer's Dementia. The large variation in factors responsible for the cognitive decline that can accompany aging increases the difficulty to develop drugs to treat this condition. Also, as mentioned, it is still largely unknown to what extent which factors contribute to unsuccessful aging.

Models of cognitive dysfunction can be used to gain insight in the etiology and treatment of agerelated cognitive decline and dementia. In a model, first, a decline in cagnitive performance, such as seen in the aged or demented is induced in thealthy volunteers through biochemical or physiological methods. Second, a putative drug or cognition enhancer is tested in its potential to reverse the cognitive deficiency. Third, the model needs to be evaluated in terms of the predictiwe validity with regard to the drug effect in the target population. By manipulating and modulating one aspect of aging, maximum control can be exerted in the experimental setting, which increxses the value of the findings. Furthermore, this method is less expensive and less time consuming compared to studying the target population of elderly people. Due to the controlled testing of homogenous groups, less subjects are needed. Lastly, a model which has a good prediclive validity 
may give ansight in which factors contribute to unsuccessful aging and how, and which drugs could be deweloped to treal age-related cognitive deficiencies. In recent years, a number of nodels of age-related cognitive decline and dementia have been used. An emphasis has been laid on two basic factors. These factors, which are thought to contribute to the cognitive deficiency in aging, are brain hypoxia due to atherosclerotic processes and neurotransmitter deficiencies.

In the present paper, first, the functional changes in cognitive performance in normal aging, agerelated cognitive deficiency, and dementia and the factors thought to be important in normal and unsuccessful aging are reviewed. Then, the modelling of these factors is discussed in terms of the background of the models, their cognitive effects, the effects of drugs used in the models and the predictive value of the model, i.e. the outcome of drug studies in the target population. This is followed by discussion of the plausible mechanisms involved and a general discussion of the possibilities of use of the model. Thus, the focus of the present article lies upon the possibilities in the use of models for the investigation of cognition enhancers in age-related cognitive deficiency and dementia.

\subsection{Cognitive changes în aging, age-related cognitive decline, and dementia.}

\subsubsection{Normal cognitive aging}

Normal aging does not necessarily involve the deterioration of highly overlearned functions (atutomatic tasks or procedural memory) or of knowledge that is deeply processed (semantic memory) (Huppert, 1991; Salmaso, 1993). Poon (1993) argued that subtle changes in memory functioning of elderly indiwiduals are quite difficult to detect, since many factors (like current health status, intelligence and experience, but also environment and task type) can influence memory performance in individuals. Indeed, about 50\% of elderly subjects investigated in a population sample did not perform conform the subjects of their age cohort and were either much worse or much better (Smith \& Baltes, 1993). By comparison, a reliable profile of performance can be seen with a systematic slowing of cognitive processes in normal aging and early dementia. A 36\% slowing of attention, decision making, memory retrieval, verbal and spatial tasks was seen in 60 year old as compared to 20 year old (Poon, 1993). Elderly subjects perform especially slower and /or less accurately, as compared to younger subjects, on complex or novel tasks which have to be done under the pressure of time (Fozard, Vercryssen, Reynolds, Hancock, \& Quilter, 1994; Houx \& Jolles, 1993; Houx, Vreeling, \& Jolles, 1993; Kok, Looren de Jong, \& Van Rooy, 1988). According to a model by Hasher \& Zacks (1979) with age, there is an increase in the sensitivity to perform difficult tasks which require effortful information processing. These tasks require attention and demand energetic capacity, or resources. Task complexity can then be operationalized in the additional demand that is made upon the functional information processing capacity or the mental "resources" (Kok et al. 1988). This is seen in tasks which require the dividing of attention, for instance, or in tasks that require the inhibition of distracting information (Carlson, Hasher, Connely, \& Zacks, 1995; Houx et al., 1993)

Also, a decrement in some, but not all, aspects of memory functioning can be noted (Huppert, 1991; Salmaso, 1993; Verthaeghen, Marcoen, \& Goossens, 1993). When compared to the performance of young adults, elderly persons tend to be poorer in the acquisition and retrieval of new information (secondary memory) and in the manipulation of information that is held in memory (working or primary memory). For instance, a meta-analysis by Verhaegen. Marcoen and Goosens (1993) of 122 relevant research papers revealed that, in general, elderly subjects have more problems with list recall, especially if the lists consist of unorganized material. Also, the slope of the Memory Scanning Test, reflecting the speed of search in working memory, is markedly 
higher in the elderly. Salthouse (1985) suggested that short tem menory ot working memory capacity is especially sensitive to age since it reflects the decrease in "resources" avalable in aging: Also Craik (1986) hypothesized that fewer processing resources reduce the efficiency and efficacy on seff-initiated, voluntary, and effortful memory tasks, such as retrieval of information from long term memory and manipulation of information in working memory.

The impaired manipulation and organization of information in working memory in elderly subjects and the slowing of complex task performance lead to the conclusion that aging is associated with a decrease in information processing capacity or mental resources (Jolles et at., (995b).

\subsubsection{Cognitive changes in age-associated cognitive decline and dementia}

There is a wide range from successful cognitive aging to pathological conditions, such ats Alzheimer's Disease, which represent age associated cognitive changes across a continuum (see: (Rediess \& Caine, 1996). Successful aging is defined by a cognitive performance alsove average as compared to age peers, but within the normal range for younger adults. Also within the normal range are elderly with Age-Associated Memory Impairment (AAMI, Crook et al., 1986) who have a memory performance at least I SD below the mean established for young adults on a standardized secondary memory test. The borderline conditions between normal cognitive aging and dementia consist of people with objective cognitive impaiment as compared to aged peers. The DSM-IV (APA, 1994) includes two new conditions to describe this bordlerline group, i.e. the Age-Related Cognitive Decline (ARCD) and the Mild Neurocognitive Disorder. ARCD is characterized by cognitive deficits apparent when subjects are compared with the average cognitive performance (below 1 SD) of aged peers, when adjusted for education (Levy, 1994). The objectively identified cognitive decline in subjects complaining of memory and attentional dysfunctions should thus be within the normal limits, and should not cause a significant functional interference in daily activities. However, this condition may be a focus of clinical attention. The condition should also not be attributable to medical (e.g. cerebral or systemic disease); neurological (e.g. postencephalitic, postconcussiomal syndrome); or psychiatric (e.g. anxiety, depression) conditions or centrally acting drugs known to induce cognitive impairment, By contrast, the Mild Neurocognitive Disorder is defined as a decline in cognitive functioning from premorbid levels which disrupts social and occupational functioning, but which is not severe enough to warrant the diagnosis of dementia. The diagnosis of Mild Neurocognitive Disorder requires a specific medical condition to be identified as etiologically related to the cognitive impairment (e.g. neurological or other medical condition). Subjects in the early phasese of dementia can be diagnosed as having a Mild Neurocognitive Disorder (Rediess \& Caine, 1996). In these early phases, qualitatively the changes in information processing are similar to those in normal aging, save for significant memory consolidation deficits (Jolles, 1986) although this is not necessary for the diagnosis Mild Neurocognitive Disorder. For the diagnosis Dementia of the Alzheimer's Type, memory impairment is obligatory with one or more other cognitive deficiency and a significant impairment in social or occupational functioning. There should be no other medical condition responsible for the cognitive deficiencies objectified. Thus, in patients suffering from dementia, the functional changes are much more widespread, as is seen in the gradual decrement of memory processes, planning and many other cognitive processes as well as emotional and social behaviors (Jolles et al, 1995b).

Hence, the criteria of the AAMI (Crook et al., 1986) and ARCD condition exclude patients with physical, neurological or psychiatric disorders that are suspected to cause cognitive deficiencies, while the criteria of the Mild Neurocognitive Disorder state that one of these conditions should be 
objectuted from physical examination or laboratory findings. Thus, parients afficted with this conditon maty include victims of Biological Life Events (BLE) (see: Houx, Vreeling, Jolles, 1989), patients with physical conditions (e.g. illness) affecting: their cognitive performance, psychiatric patients, or subjects using drugs that may affect their cognitive performance, but also elderly in the early prodromal phase of dementia (Jolles et al., 1995a). Elderly that have experienced adwerse health related factors or Biological Life Events (BLE), such as neurologic or systemic disease, general anaesthesia, closed head injury, and excessive use of alcohot or psychotropic drugs were indeed found to show a more impaired memory performance and an additional sllowing on complex task performance as compared to controls (Houx \& Jolles, 1993; Houx \& Jolles, 1994; Houx et al., 1991). In the Maastricht Aging Study (MAAS), a large scale cross sectional and longitudinal study of cognitive aging including over 2000 subjects, various BLE were tested separately in their effect on cognitive functioning. As for systemic disease, both insulin dependent and non-dependent diabetes and chronic bronchitis were associated with accelerated age-related decline in cognitive ability. Cardiovascular disease (including hypertension) was unrelated to test performance (Boxtel van. Buntinx, Houx, Metsemakers, \& Jolles, submitted). Also, Dijkstra (1997) showed that general anesthesia did not have long term effects on cognitive dysfunction, although elderly subjects did experience short term detrimental effects on cognitive functions. In patients with mild closed head injury, however, at slowing of performance and a decreased memory performance was seen, as compared to healthy controls (Klein, Houx, \& Jolles, 1996). In fact, patients with diabetes or mild head injury had a cognitive age of subjects 10-15 years their chronological age. The long term use of benzodiazepines and alcohol have shown detrimental to cognitive performance (Gorenstein, Bernik, Pompéia, \& Marcourakis, 1995; Molina et al, 1994). Yet, in normal population studies chronic alcohol use was not seen to accelerate cognitive decline (Boxtel van et all, submitted; Desmond, Tatemichi, Paik. \& Stern, 1993). It is thus of major relevance to investigate which factors may contribute to accelerated aging in more detail, since this may ultimately affect the choice of treatment for the heterogeneous group of patients afflicted with Mild Neurocognitive Disorder.

\subsubsection{Neurobiological correlates of Brain Reserve Capacity in aging and dementia}

The implicit assumption in research of accelerated cognitive aging and BLE is based upon the concept of Brain Reserve Capacity (BRC), which is concerned with the capacity of functional brain tissue. The central processing capacity hence is assumed to be similar to the concept of "resources'. The concept of BRC (Mortimer, 1994; Satz, 1993) presumes that there may be protective factors, such as intelligence, certain diets, physical fitness, or drugs which could preserve brain capacity. Vulnerability factors, such as genetic components, some BLE or health related factors as well as current or former use of behavioral toxic psychoactive drugs, and age-related changes in the brain could accumulate in at reduction of BRC which will eventually become apparent as $A$ ACD or dementa when a critical threshold is reached. Evidence for neurobiological correlates of BRC are brain size, cortical surface area, and metabolic and neuronal efficiency (Satz, 1993). In aging, the brain's functional capacity changes. This can be seen anatomically, in the atrophy of neural systems and the dilatation in ventricles (Joseph, 1992; Selkoe, 1993). Biochemically, it is reflected in the decreased cerebral metabolism (De Reuck et al, 1992; Smith, 1984) and the reduction in the synthesis of neurotransmitters in the brain (Wallin, \& Gottfries, 1990).

A reduction in cerebral perfusion is seen in aging and has long been suspected to be a crucial factor in unsuccessful aging (Stern, Alexander, Prohovnik, \& Mayeux, 1991). Most studies have shown that in the general population there is a decrease in general blood flow and oxygen 
consumption with age. However, the decrease in cerebral blood flow (CBF) and in cerebral oxygan consumption is mainly seen in elderly subjects with arteriosclerosis. In healthy elderly subjects (an: atypical subgroup) without hypertension or other vascular disense only a reduction in cerebral glucose consumption is seen, without an aceompanying decrease in oxygen consumption (Smith. 1984). In $\mathrm{AD}$, a strong reduction in the glucose metabolism is seen. A reduction of ATP formation from $7 \%-20 \%$ in incipient and progressively advancing $A D_{3}$ up to $50 \%$ in stable advanced $\mathrm{AD}$ was seen. The cerebral metabolic tate of oxygen was only slightly or not at all diminished att the begirning of this disorder (Hoyer, 1992). The occurrence and severity of dementia appears to be correlated with the decrease in blood flow and oxygen metabolism in the frontal, partictal and. temporal cortex (De Reuck et al., 1992). According to Baron and Marchal (1992) in degenerative. dementias (particularly Alzheimer's disease) the hypometabolism preferentially affects the associatiwe cortex. Further, they proposed that the cumulative effects on cortical functioning of multiple wascular lesions, superimposed on degenerative neuron loss, might account for age-related cognitive disorders in "normal" aging as well as in the so-called "vascular" or "mixed" dementias. A reduction in cerebral blood flow (ischemia) can lead to brain thypoxia (lack of oxygen) and eventually in the production of free radicals and cell atrophy. When the oxygen and glucose availability decrease a cascade of events occur leading to cell death. For instance, excitotoxins, like glutamate, stimulate a toxic influx of calcium $\left(\mathrm{Ca}^{2+}\right)$ and as such are involved in the pathway leading to cell death (Pujol et al., 1990; Weinachter, Blavet, O'Donnell, Mackenzie, Rapin, 1990).

Furthermore, research has shown that, in aging, the decline in neurotransmitter function is probably due to the loss of neuronal cell bodies located in the brain stem, such as the locus. cereleus and substantia nigra (Colleman \& Flood, 1987). The decline in cholinergic functions implicated in the age-related cognitive dysfunction and dementia specifically is well described (e.g. Kopelman, 1986). A review by McEntee \& Crook (1990) describes that substantive evidence indicates a decline in brain catecholamines in aging and dementia. There is limited evidence for changes in serotonin (5-HT) functioning in normal aging. In contrast, in AD, 5-HT function clearly is abnormal (McEntee \& Crook, 1991). In a vascular dementia (VD) group a similar pattern of (monamine) neurotransmitter changes was seen, possibly indicating a common pathway of in the two major forms of dementia, particularly in the later stages (Wallin \& Gotfries, 1990).

\subsection{Models of brain hypoxia}

\subsubsection{Hypoxia model}

\section{Background}

The hypoxia (lit.: low oxygen) model was developed as a method to test putative cognition enhancing drugs (Maier-Lenz, Ringwelski, Klein, \& Richter, 1980). It has been considered 1o be a model for cerebral ischemic disorders (lit: lack of blood, e.g. due to circulatory deficiencies) (Kraajer, 1988). A reduction in cerebral perfusion is seen in aging and has long been suspected to be a crucial factor in unsuccessful aging (Stern et all., 1991). Inducing hypoxia, by letting subjects breath air with a low oxygen content or by thesting subjects at (simulated) high altitudes, has been used as a model to test the therapeutic efficacy of nootropic drugs since the early eighties (Kraaier, 1988; Saletu \& Grünberger, 1984; Schaffer, Hörmann, \& Amold, 1981; Schaffler, \& Kiliusnitzer, 1988; Schaffler \& Reeh, 1985)

\section{Cognitive effects}

As for the effect of hypoxia in inducing cognitive dysfunction, its effect has long been described as a 'dulling of the senses' (Haldane, Kellas, \& Kennaway, 1919). Whether this effect is a central 
'lethargy' effect, which may iready occur at oxygen concentrations ( $\pm 15 \%$ oxygen in air) equivalent to $2970-3500 \mathrm{~m}$ (Forester, 1985) or a peripheral effect, in a disruption of some part of the visual system (Fowler, Taylor, \& Porlier, 1987; Gibson, Pulsinelli, Blass, \& Duffy, 1981) is molear. However, mild thypoxia ( $\pm 11-14.5 \%$ oxygen in air, comparable to an allitude of 3048 m $4700 \mathrm{~m}$ ) affects both the visual sensitivity and the performance in reaction time tasks that do not require peripheral visual sensitwity (Fowler et al., 1987; Kida \& Imai, 1993; O'Hanlon \& Horvath 1973). In contrast, one study reported a small improwement in visual perception function after a real hypoxic challenge, i.e, a rapid transport $103450 \mathrm{~m}$. Possibly this effect was due to the compensatory increase in HR and cerebral blood flow (Schlaepfer, Bartsch, \& Fisch, 1992). Also, some subjects can perform RT lasks whout difficulty up to a simulated altitude of $6000 \mathrm{~m}$ (oxygen level $9.8 \%$ ) which suggests an individual susceptibility to hypoxia (Kida \& Imai, 1993),

The effects of mild hypoxia on memory functions are disputed (Crow \& Kelman, 1971; Denison, Ledwith \& Poulton, 1966; Fowler, Paul, Porlier, Elcombe, \& Taylor, 1985). Studies that evaluated the effect of hypoxia on specific information processing stages employed the Sternberg paradigm or specific EEG parameters (Ewoked Potentials, such as P300). These studies showed that mild to moderate hypoxia affects sensory discrimination and evaluation processes (N2 and P300 components) at a central level, rather than affect memory processes directly (Fowler, Prlic, \& Brabant, 1994; Kida \& Imai, 1993; Wesensten et al., 1993). At oxygen concentrations higher than - or equivalent to - an altitude of approximately $5000 \mathrm{~m}$, hypoxia has shown marked effects in terms of memory dysfunctioning (Ernsting, 1978).

However, the risk for adverse vegetative and cardiovascular side-effects, such as rhythm disturbances on syncope with bradycardia, increases at (simulated) altitudes of approximately 4600 to $6000 \mathrm{~m}$ (Kida \& Imai, 1993; Kraaier, 1988; O'Hanlon \& Horvath, 1973; Saletu \& Grünberger, 1984). Prolonged ( 115 days) exposure, acquited by mountaineering expeditions at these high altitudes $(5000 \mathrm{~m}$ and over) has yielded demonstrable residual (after 75 days from descent) cognitive dysfunction (memory, concentration). Again, significant differences between subjects were found, which confirms the individual susceptibility to hypoxia (Cavaletti \& Tredici, 1993). In this line, many others did not find residual effects of prolonged exposure to high altitude (see : Bahrke \& Shukitt, 1993 for a review). The individual susceptibility to hypoxia may thus resemble the neuroprotective status of a subject's brain.

Drug studies

Many studies have been used to evaluate possible drug effects in counteracting the effect of hypoxia. For instance, aniracetan and piracetam (Kraaier, 1988; Saletu \& Grünberger, 1984; Zyhlarz et al. 1994); the piracetam analogue BMS-181168, (Saletu et al., 1994), cyclandelate (Schaffer et al, 1981); hydergine or co-dergocrine mesylate and nicergoline (Saletu, Grunberger, \& Anderer, 1990; Schaffler K Klausnizer, 1988); gingko biloba (Schaffler \& Reeh, 1985); and the new drugs riluzole (Salem, Grunberger, Anderer, \& Linzmayer, 1996a; Saletu, Grunberger, Anderer, \& Linzmayer, 1996b), actovegin (Schaffler, Wauschkuhn, \& Hauser, 1991) and caroverine (a calcium channel blocker with antiglumatinergic properties) (Saletu, Grunberger, Anderer, Linzmayer, \& Konig, 1996c) all have been shown to attenuate or reverse hypoxianinduced cognitive dysfunction.

Predictive validity

As for the predictive value of this model, piracetam analogues have shown only limited therapeutic value in reversing cognitive dysfunction in age-associated cognitive decline (AACD). For instance, in 3 studies piracetam was shown to have a modest positive effect in AACD (Fioravanti et al., 1991; Israel, Melac, Milinkevitch, \& Dubos, 1994; Schmidt, Brendemuhl, Engels, Schenk, \& Ludemann, 1991) while one (Riedel, Peters, Van Boxtel, \& O'Hanlon, 1995b) found no effect of piracetam. 
Also, there is no consistent evidence for a beneficial effect of the drug on cognitive function in AD. Yet, one study suggested that higher doses of piracetam might slow the rate of cognitive deterioration in AD, especially on memory functions (Croisile et al., 1993). Data on aniracetam in the treatment of $\mathrm{AD}$ are also inconclusive. One study failled to show an effect of aniracetam in AD patients (Sourander, Portin, Molsa, \& al; 1987), while another multicenter clinical study did find that aniracetam was superior to placebo in AD patients (Senin et al., 1991).

Several studies with AACD patients revealed a significant effect of hydergine on cognitive function. However, it should be noted that an effect of hydergine was only seen in some, but not in most patients (Goodnick, \& Gershon, 1984; Wadworth, C Chrisp, 1992). Also, according to Spiegel (1995), in demented patients, hydergine has been shown to increase the cerebral blood: flow (CBF) and has an effect on mood and behavior, but not on memory and intellectual functions. Gingko biloba has been shown to have some long term protective effects (Kleijnen \& Knipschild, 1992). In middle-aged subjects complaining of mental insufficiency, a combination of gingko biloba and ginseng was seen to improve cognitiwe functions and physical fitness (Wesnes" 1996).

\section{Mechanisms}

According to some authors, the validity of the hypoxia model is questionable since the reversal of the cognitive deficits induced by hypoxia may involve mechanisms which are irrelevant to the disease one ultimately wants to treat, e.g. brain ischemia (Hall, Puech, Schaffler, Wesnes, \& Gamzu 1990). For instance, according to Gamzu, Birkhimer, Hoover, and Gracon (1990) hypoxia induced cognitive dysfunction is often reversible without pharmacological intervention. Compensatory increases in cerebral blood flow have been reported to occur during hypoxia (Ellingsen, Hauge. Nicolaysen, Thiresen, \& Walløe, 1987). Yet, it should be kept in mind that even if compensatory effects take place during the breathing of hypoxic air, the amount of oxygen in the blood still remains low. Research shows that some brain regions (e.g. hippocampus CAI cells) are especially vulnerable to relatively small decreases in the availability of oxygen (Aggelton, 1996). In this line, Huppert (1982) found that the degree of memory impairnent was positively associated with the degree of oxygen saturation in arterial blood samples. Furthermore, a reduction in axygen levels: decreases catecholamines and serotonin in rat brain, since these neurotransmitters require the direct utilization of oxygen (Davis, Giron, Stanton, \& Maury, 1979; Gibson et al., 11981). Also, a decreased synthesis of acetylcholine has been found to occur in hypoxia (Blass \& Gibson, 1979).

However, increased cognitive effort and training have also been seen to overcome hypoxiainduced cognitive dysfunction (Bahrke, \& Shukitt, 1993). Simillar effects can be found in sleep deprivation studies. Bills (1937) compared states of fatigue and reduced oxygen availlability in terms of their effects on the Color Naming test and argued that the physiological basis of mental fatigue is, in part at least, accounted for by the reduction of the available oxygen to the brain.

Discussion

In generall, cognition enhancers that can be tested with the hypoxia model should have an effect on vasodilation" improve CBF, and/ or influence the cerebral metabolism. For instance, drugs that should reverse effects of hypoxia are nimodipine and pyritinol. Nimodipine, al Cata antagonisl., is known to improve CBF (De Jonge \& Traber, 1993). A review by Grobe-Eingler (1993) describes two studies reporting that nimodipine improved cognitive function on top of regular mental

1Lader (1985) described several vasoditators. For instance, xanthines and nitrites have valsodilator effects but lower the systemic blood pressure so that CBF is impaired rather than enhanced. New vasodilators; which claim to improve CBF, are papaverine, cyclandelate, isoxsuprine (all beta-adnentergic agonistsi and nafidrofury. Cosaldon containis nicolinic acid which increases CBE. 
exerctse and facilitated learning in elderly whth anganic brain syndrome. Other studies indicated a possible delayed progression of $\mathrm{AD}$. Pyritinol, a $\mathrm{B} 6$ (pyridoxine) derivative is believed to anhance glucose utilization and to increase $\mathrm{CBF}$ and has been shown to improve vigilance in humans (Fischoff et al, 1992). According to Gamzu et al. (1990) testing drugs during induced hypoxia does not predict clinical efficacy. Yet, they concluded that the hypoxia model can be of value in that the changes produced by hypoxia are reflected in the changes of many neurotransmiter levels (DA, NE, ACh, 5-HT). A positive outcome might then define a dose range for future studies while a regative outcome would not necessarily halt development.

Lastly, drugs deweloped to protect against the cell damaging effects of brain ischemia (e.g. antioxidants) cannot be tested in hypoxic models with humans. In animal research injuries to the brain have been made through the use of ischaemia, hypoxia, hypoglycemia or electric shock (Hollister, 1990). Examples of these drugs that can be tested in these animal models are antagonists of the NMDA receptor, such as selfotel and eliprodil (Vender, Nair, \& Lehmann, 1995), and calcium antagonists (Flint Beal, 1992). Adenosine reuptake inhibitors are also thought to have neuroprotective effects during ischemia. The transmitter adenosine can be triggered by EAA and reduces the $\mathrm{Ca}^{2+}$ influx. Adenosine agonists are propentofylline and, possibly, vinpocetine (Pertuche Krieglstein, 1993). However, although protective effects of these drugs have been seen in animal models of hypoxia, it has proven difficult to generate these findings to humans with memory impaiment (Weinachter et al., 1990). Hypoxia in humans thus is not a model for cerebral ischemic damage, but for the effects of the decrease in the oxygen availability and the concomitant decrease in neurotransmitter synthesis which accurs in aging due to atherosclerosis and other deficiencies in the circulatory system. Since hypoxia may indirectly affect neurotransmitter systems, other models, such as the scopolamine model (see par. 2.4.1), can be used concomitantly to test whether the effect of the drugs may be primarily in enhancing neurotransmitter levels.

\subsubsection{The hyperyentilation model}

\section{Background}

Kraaier, van Huffelen \& Wieneke $(1988 ; 1989)$ stated that since in hypoxia compensatory increases in blood flow are seen to occur, hyperventilation (HV) should be considered a more adequate and safe human model of reversible brain ischaemiat. The decrease in $\mathrm{pCO}_{2}$ which occurs during hyperventilation is known to lead to vasoconstriction of the precapillary arterioles and a redtuction of cerebral blood flow of about $40-60 \%$. Also, hyperventilation has been found to affect EEG (Kraier et al, 1988) and short term or working memory functions (Kraaier et al., 1989; Rother, Kesslet, Funke, Rother, Windisch, 1994). However in both studies no control procedures werte used and in the study by Kraaier no statistical evaluation on the working memory memory (STM) findings was given.

\section{Cogninve effects}

In earlier studies, HV has been reported to provoke a decline in several aspects of cognitive functioning, such as STM (Rathn at al., 1946), motor-coordination (Balke \& Lillehei, 1956; Gibson, 1978; Rushmer, Boothby, \& Hinshaw, 1941), choice reaction time (Stoddart, 1967), and character detection (Niklokov, Patchev, Kisselkova, \& Harleva, 1991). By contrast, also an absence of effect of HV has been found, e.g. on the Wechster memory test (Murrin \& Nagarajan, 1974) and on wistall discrimination (Rahn ef al ", 1946). These inconsistencies may be due to methodological differences, for example, control procedures were often not used (Balke, \& Lillehei, 1956; Kraaier et al., 1989; Rahn et al. 1946; Rother et al., 1994; Rushmer et al., 1941). In a study by Hogervorst et al (submitted) normocapnic hyperventilation used as a control procedure was seen to decrease psychomotor speed. An additional effect of hypocapnic hyperventilation was 
only seen on the response times of a wisual sensitivity task. No effects were seen on memory functions.

\section{Drug studies}

In the study by Kraaier et al, (1989), 3-OH aniracetam was able to reverse the hyperventilation induced qEEG changes without producing cerebral vasodilation. In the study by flogervorst; al (submitted), caffeine was seen to decrease both working and long term memory functions in hyperventilation and its control procedure, normocapnit. No other studies have been done which evaluated drugs in the hyperventilation model.

\section{Mechanisms and discussion}

Some authors question the cognitive effects of hyperventilation (Garssen, 1986; Hogervorst et al). The supposedly hyperventilation-induced cognitive impairments have been atributed to overbreathing itself, or cramps that may accur during hyperventilation, and which may hinder the adequate performance of (psychomotor) tasks. However, in the study by Hogervorst et al, both hyperventilation and its control procedure did not affect the speed of movement. This saggests that symptoms accurring during hyperventilation (shortness of breath, headache) or having to overbreath in a fixed rhythm simultaneuosly may have interfered with cognitive performance. However, since overbreathing itself was seen to induce a strong slowing of response times, hyperventilation could be used as a model for the increased neural noise, or the unrelated neural activity that interferes with information processing, which according to some authors (Salthouse \& Lichty, 1985) may be an important factor in aging. Also, although a reduction in cerebral blood how is seen to occur during hyperventilation, it is unclear whether hypoxia actually oceurs in the brain during hyperventilation (Albrecht, Miletich, \& Ruttle, 1996; Kennealy, McLennan, Loudon, \& McLaurin, 1980). Namely, hyperventilation also has shown compensatory vasodilator effects, similar to hypoxia, within approximately 10 min (Ellingsen et al. 1987).

Furthermore, Balke and Lillehei (1956) mentioned a state of apathy to occur in hyperventillation, concomitant with a decreased responsiveness to external stimuli. This state could be attenuated by strong instructions to focus upon the task. Thus, effort and motivational factors similarly to hypoxia, can be seen to overcome deficits. Central fatigue may occur due to a relative ischemia. One explanation is that the increase in excitation and firing frequency of neurons occurring in hyperventilation could have been responsible for central fatigue occurring after a prolonged period of testing and a resulting lack of regenerative capacity of netrons (Garssen, 1986). An alternative explanation is that the decrease in $\mathrm{CO}_{2}$ might have been responsible for other changes in the brain. For instance, acetylcholine synthesis can be seen to decrease with a fall in $\mathrm{CO}_{2}$ production (Blass, \& Gibson, 1979). A reduction of the cholinergic actiwity induces cognilive deficiencies (Bartus, Dean, Beer, \& Lippa, 1982). These mechanisms would explain why an increase in the response times of the viswal sensitivity task (the last task) occurred in our stlidy (Hogervorst et al, submitted). Yet, due to its limitations (long duration of onset of action, many aspecific effects), the hyperventilation model does not seem a model of first choice in testing putative cognition enhancers.

\subsection{Neurotransmitter models}

Neurotransmitters such as acetylcholine, GABA, serotonin, norepinephrine, dopamine etc. are thought to have a modulatory influence on memory and cognition. As such, they may be implicated in mimicking or treating agemassociated cognitive decline or AD (Squire, 1987). Manipulation of neurotransmitters, and more specifically, acetylcholine, remains the most extensively studied strategy against Alzheimer's Disease (Nordberg, 1996). 


\subsubsection{The scopolamine model}

\section{Background}

Scopolamine induced cognitive impaiment is considered to be a model of age-related cognitive deficiencies and more specifically of Alzheimer's dementia, since it models the cholinergic deficiency thought to be implicated in these processes (Bartus et al, 1982). Biochemical studies in $\mathrm{AD}$ patients have shown that the activity of choline-acetyltransferase (CAT) is reduced in the caudate nucleus and hippocampus, which results in al lowered synthesis of acetylcholine $(\mathrm{ACh})$. Cholinergic neurons in the nucleus basalis allso have been found to be reduced in AD (Davies \& Maloney, 1976). Fundamental research into the cholinergic hypothesis of aging showed that cholinergic deficiencies created by the inhibition of choline uptake by hemicholinium-3 and the subsequent blockade of acetylcholine (Ach) synthesis, or by specific lesions in the nucleus basalis of Meynert produce defective learning in animals (Hollister, 1990). Also, a cholinergic blockade by scopolamine, a cholinergic antagonist, causes a variety of memory deficits that resemble the deficits seen in AD patients (Kopelman, 1986). These deficiencies can be reversed by acetylcholinesterase inhibitors, such as physostigmine, and muscarinic agonists, such as arecoline (Briley, 1990).

Cognitive effects

Ghoneim \& Mewald (1977) earlier showed that the scopolamine-induced amnesia was partly reversed by physostignine while the diazepam induced amnesia was not. Physostigmine inhibits acetylcholine-esterase, thereby increasing ACh availability. Drachman (1977) further found that the scopolamine-induced annesia could not be reversed by dextro-amphetamine (a stimulant acting upon monoaminergic systems). These findings pointed to a specific relationship between central cholinergic activity and memory. However, scopolamine in later studies has been shown to negatively affect the early processing of information, e.g. stimulus evaluation and identification (Brandeis, Naylor, Halliday, Callaway, \& Yano, 1992; Callaway, 1984; Wesnes \& Warburton, 1983). Hence, memory processes may be affected indirectly rather than directly. Furthermore, we showed that, although scopolamine affected memory and several cognitive and motor speed parameters, no effect was seen on age-sensitive complex tasks requiring executive functions, such as response inhibition (Riedel, Hogervorst, Verhey, \& Jolles, 1997). Also, the scopolamine induced memory deficiency in this study resembled the normal age-associated, but not the AD related. decrements in memory performance.

Drug studies

On the premise that cholinergic neurons and receptors are preserved to some extent, administration of ACh analogues should counteract ACh deficiency effects. However, most cholinergic precursors do not pass the blood brain barrier (BBB) or have intolerable side-effects (Lader, 1985). Cholinesterase inhibitors, such as physostigmine, velnacrine, and tacrine prevent the breakdown of ACh in the synaps. This type of drugs has shown positive effects in cholinergic deficiencies. For instance, physostigmine has been shown to improve the decreased stimulus detection performance in vigilance-like situations after scopolamine had been adminstered (Warburton \& Brown, 1972). Also velnacrine was shown to reverse cognitive defects in the scopolamine model (Wesnes, Simpson, Christmas, \& Siegfried, 1990b).

Nicotine is a nicotinic cholinergic agonist. Another study by Wesnes and Revell (1984) in young human volunteers showed that the effects of scopolamine (1.2 mg p.o.) were antagonized by nicotine. In this study, $1.5 \mathrm{mg}$ of nicotine was shown to improve the performance (speed and accuracy) of a rapid intormation processing task, while it reduced the Stroop effect (distractibility). Nicotine thus improved stimulus selection and sustained concentration. In another study, nicotine attenuated the scopolamine impaired storage in short term memory, the slowing of 
speed of working memory memory scanning, and the indnced slowing of reaction time. In addition, caffeme was able to reverse the scopolamine induced memory (short and long tem recall) and visual perceptual sensitiwity dysfunctions (Riedel et al., 1995a).

Predictive value

First, a review of 17 studies conducted between 1977 and 1982 revealed that there is little to no therapeutic efficacy of treatment with choline in $\mathrm{AD}$ patients (Bartus et al., 1982). As for the predictive value of the scopolamine model, only a short lived enhancement of memory ftunctions could be seen when physostigmine in $\mathrm{AD}$ patients was given intravenous (Briley, 1990; Pathy, 1993). Hence, physostigmine has a short half life and also several side-effects which limits its practical use. In AD patients velmacrine was superior to placebo on cognitive function and global assessment scales (Antuono, 1995; Siegfried, 1993). However, velnacrine was shown to have adverse effects on liver function in about a third of the subjects treated, which lead to discontinuation of the treatment (Antuono, 1995). Velnacrine's development was discontinued in 1994. Nordberg et at (1992) have found improvements of tacrine in cholinergic function and brain glucose metabolism in Alzheimer patients when given over a 9 month period. Wilcock and Harold (1996) estimated a $30-40 \%$ efficacy of tacrine in patients with mild to moderate dementia. Holford and Peace (1994) also showed an improvement in $\pm 30 \%$ of the demented patients and also found that lecithin, a cholinergic precursor, had a small additional beneficial affect, independent of tacrine. Yet, another placebo controlled crossover study with Alzheimer patients found that tacrine improved attentional, rather than memory functions (Sahakian et al., 1993).

Nicotine has shown transitory memory enhancement in healthy subjects, but, according to Pathy (1993) not in AD patients. Yet, also positive effects of nicotine in Alzheimer patients in inproving attention, but not memory, have been found (Jones, Sahakian, Levy, Warburton, \& Gray, 1992; Newhouse et al., 1988; Sahakian, Jones, Levy, Gray, \& Warburton, 1989) Positive effects of nicotine in healthy, middle-aged subjects were reported (Hogerworst, Riedel, Rozendaal, Van Boxtel, \& Jolles, 1995). Caffeine has been noted to have dose-dependent positive effects on cognitive performance, especially in the elderly (Jarvis, 1993).

Mechanisms

The precursor choline thus has shown very limited therapeutic value in the treatment of $A D$. According to some authors this was probably due to the extensive loss in cholinergic neurons (Sunderland et al. 1987). For instance, nicotine receptors thave been found to be reduced in Alzheimer"s Disease (Nordberg. Hertvig Lilja, \& al, 1990). It is in general accepted that there is no difference between normals and $\mathrm{AD}$ patients in muscarinic receptor density, since also during normal aging, there is a decrease in muscarinic (cholinergic) receptor density (Nordberg, 1996). Some controversies remains in that some studies have found an exaggerated decrease in muscarinic receptors in the hippocampus in AD (Bartus et al., 1982).

Furthermore, although the predictive value of the model seems adequate, some authors question the specificity of the scopolamine model (Gamzu et al., 1990). For instance, investigators found that methamphetamine, which acts upon the monoaminergic system, could reverse the scopolamine effect on word recall in young healthy human wolunteers (Mewaldt, \& Ghoneim, 1979). Stimulants, such as methamphetamine, are known to positively affect learning and memory and may even directly affect consolidation processes (Soetens, Casaer, D'Hooge, \& Hueting, 1995; Soetens, D'Hooge, \& Hueting, 1993).

Also, vitamin BI (thiamine), a TRH analog, the NMDA antagonist D-cycloserine, the MAOinhibitor moclobemide, and 5-HT3 antagonists (granisetron, ondansetron) have all been found to attenuate the scopollamine effect (see: Riedel, \& Jolles, 1996 for a review). Still, Schwartz. Hashtroudi, Herting, Schwartz, and Deutsch (1996) found that $15 \mathrm{mg}$ of D-cycloserine improved 
memory in patients with mild to moderate $A D$, although earlier studies found no effect of D-cycloserine in AD patients (Mohr, Knott, Herting, \& Mendis, 1993). Moclobemide has also shown beneficial effects on memory in non-impaired elderly volunteers (Wesnes, Simpson, Christmas, Anand, \& McClelland, 1989). In addition, Angiotensin Converting Enzyme (ACE) inhibitors; such as captoprit, hawe shown to rewerse scopolamine induced cognitive impairment in animal studies (Sharma \&ukami, 1992). Captopril was shown in a double blind study with AD patients to enhance cognitive performance on the Trail Making Test (in: Costall, Barnes, Hamon, Muller, \& Briley, 1990). Enalapril, another ACE inhibitor, however, could not reverse the scopolamine effects in human volunteers (Lines, Preston, Dawson, Brazell, \& Traub, 1991)

Furthermore, scopolamine is known to reduce the cerebral blood flow and decrease the glucose metabolism in the thalamus, the hippocampus, and the cortex (Gitelman \& Prohovnik, 1992; Honer, Probovnik, Smith, \& Lucas, 1988). Several substances may have reversed scopolamine effects through attenuating these effects. For instance, piracetam-analogues such as aniracetam or tenilsetam, may have attentated the deereased metabolism of scopolamine. A study with 26 healthy volunteers injected sub-cutaneously with $0.7 \mathrm{mg}$ scopolamine showed that aniracetam antagonized scopolamine induced decrements on memory and information processing tasks (Wesnes, Anand, Simpson, \& Christmas, 1990a). Piracetam in this study did not show an effect, probably because piracetam itself is far less potent than all of its analogues. In another study, tenilsetam, a piracetam analogue, could also attenuate the effects of scopolamine induced impairments on EEG and mental efficiency (Wesnes, Simpson, \& Kidd, 1987). According to the authors, tenilsetam in elderly subjects has been mentioned to have a favorable effect on EEG and performance.

Discussion

Scopolamine can model some of the aspects of information processing which are affected in aging. Whether it also adequately models the coggnitive detrioration seen in dementia is still controversial. Our results (Riedel, Hogervorst, Verhey, \& Jolles, 1997) indicated that the scopolamine induced memory deficiency resembled normal age-associated, but not $A D$ related decrements in memory performance. However, in this study a relatively low dose of scopolamine, as compared to other studies, was used, which may explain these findings.

Furthermore, the specificity of the madel has been questioned. Scopolamine effects were also seen to be altenuated with drugs that affect cerebral metabolism or other neurotransmitter system. This may point to the indirect actions of these systems on the cholinergic system. This indicates that scopolamine is not a model to test whether a drug has direct cholinergic actions. Models of other neurotransmitter deficiencies should be used concomitantly to test through which pathway the drug actually is effective. However, the predictive value of the scopolamine model, as was seen with cholinesterase inhibitors, direct and indirect cholinergic agonists, such as nicotine and caffeine, and non-cholinergic drugs, such as the D-cycloserine and the ACE inhibitors captopril seems adequate. Furthermore, drugs with cholinergic properties that have not been tested with the scopolamine model, have shown positive effects in the treatment $\mathrm{AD}$ which underlines the predictive value of scopolamine. A cholinergic precursor, phosphatidylserine, gave small posintive effects on cognitive functions in two placebo controlled studies with severely affected AD patients, although the overall clinical relevance of these findings was questioned (Amaducci \& group, 1988; Crook. Petrie, Wells, \& Massari, 1988). Arecoline, is a natural alkaloid with nicotinic and muscarinic agonist properties and was one of the first cholinergic agonists investigated in AD.Two studies found no effect of $i . v$. administered arecoline (Christie, Schering, Ferguson, \& Glen, 1981; Tariot et al., 1988a), while another study found that continuous infusion for five days in a placebo controlled trial significantly improved werbal memory in AD patients (Raffaele et al., 1991). Again, the muscarinic 
agonists $\mathrm{RS}-86$ and oxotremorine, which would be expected to reverse the scopolmmine effects; have not shown impressive effects in treating AD. Nor has the niotinic agonist bethanechol (Patthy, 1993).

\subsubsection{Benzodiazepine-induced amnesia model}

Background

Benzodiazepines have been reported to produce amnesia and impair other cognitive functions in animals and humans. Benzodiazepines act upon the calcium channel, which is part of the GABA receptor complex, causing a intracellular calcium flux which inhibits neuronal excitation. For instance, diazepam, a GABA receptor agonist, decreases $\mathrm{ACh}$ release in the sottex and hippocampus and may induce amnesia through this mechanism (Briley, 1990). GABA is also known to inhibit noradrenergic locus cereleus cells, the serotonergic raphe nucleus cells, and dopaminergic (substantia nigra and mesolimbic) systems (Cooper, Bloom, \& Roth, 1991).

Cognitive effects

In a study by Roache and Griffiths (1987) diazepam induced an impairment in psychomotor functions, and the immediate recall and delayed recognition memory performance. Ghoneim, Hinrichs, Chiang, and Loke (1986) also found that diazepam impaired menory performance and induced a decrease in psychomotor speed (symbol cancellation). Similarly, Matilla \& Nuotio (1983) reported that diazepam induced subjectively experienced drowsiness and mental slowness and impaired performance on psychomotor (digit symbol \& letter cancellation) tasks.

Similarly to scopolamine, diazepam has been shown to produce deficits in ordered (or item) recall of both concrete and abstract words. However, these effect were especially seen on unrelated words. Thus, according to the inwestigators, diazepam probably impairs the acquisition process and the encoding of information, rather than interfere with retention or retrieval (Frith, Richardson, Samuel, Crow, \& McKenna, 1984). Some authors state that the effects of diazepam on memory are mediated by its effects on alertness and vigilance (Frith et all, 1984; Unrug-Neervoort, Luijtelaar van, \& Coenen, 1992). Also flunitrazepam and midazolam were seen to decrease both the speed and the capacity of information processing, which was hypothesized to be due to decreased levels of arousal (Jackson, Louwerens, Cnossen, \& De Jong, 1992; Rammsayer, 1992). However, others found that midazolam induced an impaited performance on a recognition task (explicit memory) but left the perceptual facilitation in identifying degrading pictures and words (implicil task), a task sensifive for the decrease in levels of arousal, relatively unimpaired (Polster, McCartly, O'Sullivan, Gray, \& Park, 1993). The common property of the benzodiazepines mentioned here is that they all have a fast onset of action a property that has often been associated with memory deficits.

\section{Drug studies}

As for the predictive value of the benzodiazepine model, the pure benzodiazepine antagonist flumazenil has been shown to reverse benzodiazepine-induced amnesia in animals and humans, (Briley, 1990; Curran \& Birch, 1991; Ghoneim, Block, Ping, el Zahaby, \& Hinrichs, 1993; Hommer, Weingartner, \& Breier, 1993). However, flumazenil could not reverse the midazolam induced amnesia (Curran \& Birch, 1991). Also, little to no clinicall efficacy of agents that reverse benzodiazepine induced amnesia has been shown (Gamzu et al., 1990).

Caffeine, an adenosine antagonist, has been seen to reverse the effects of benzodiazepiness (Ghoneim, Hinrichs, Chiang, \& Loke, 1986; Matila \& Nuotto, 1983; Roache \& Griffiths, 1987), ws it did in the scopolamine model (Riedel et al., 1995a).

Mechanisms

One explanation for the effect of caffeine in both the scopolamine and benzodiazepine model is 
that it reversed effects through enfincing alertness and vigilance. This may be underlined by the findings that the scopolamine induced decrease of performance on the visual perceptual sensitivity task was also artenuated by caffeine (Riedel et al., 1995a). Matilla \& Nuoto (1983) showed that caffene reversed the diazepan induced subjectively experienced drowsiness and mental slowness and attenuated the diazepam impaired psychomotor (digit symbol \& letter cancellation) tasks. Yet, in another study (Roache \& Griffiths, 1987) caffene reversed the diazepam induced impairment of psychomotor functions, but could not affect the diazepan induced impairment of immediate recall and delayed recognition memory performance. Ghonem, Hinrichs, Chiang, and Loke (1986) also found that caffeine antagonized the diazepam induced decrease in symbol cancellation, but could not affect the diazepam impaired memory performance. Thus, caffeine reversed subjective and behavioral (psychomotor) effects in the benzodiazepine model but could not reverse the memory dysfunction as it did in the scopolamine model. The memory effects of benzodiazepines are most probably related to the half life, as short acting benzodiazepines with a fast absorption rate, such as diazepam, midazolam, and triazolam, induce memory impairment. while benzodiazepines with a long absorption, distribution, and elimination half life, such as flurazepam, do not have memory effects.

\section{Discussion}

The benzodiazepine induced amnesia model hence seems of a different quality as the scopolamine model, again enphasizing the lack of predictiwe validity of this model. Also, the benzodiazepine model does not seem an appropriate model for aging and $\mathrm{AD}$ because in $\mathrm{AD}$ patients postmortem reduced levels of GABA were found (in: Yates, Butterworth, Tennant, \& Gordon, 1990). However, this may be an artefact, since, according to some authors, low GABA concentrations found in $A D$ patients postmortem are almost certainly due to acidosis consequent to terminal hypoxia (Yates et al., 1990).

Beta-carbolines, antagonists or partial inverse agonists of the GABA-BZ receptor complex could be tested with this model. For instance, the beta-carboline substance $Z$ K 93426 partially antagonized most of the effects of scopolamine on memory and attention, suggesting that an interaction between the GABA-ergic and cholinergic systems is reflected in measurements of both attention and memory (Duka, Ott, Rohloff, \& Voet, 1996). Beta-carbolines have been hypothesized to be a treatment strategy for senille dementia (Sarter, Schneider, \& Stephens, 1988).

\subsubsection{Serotonergic depletion and receptor models}

\section{Backgrownd}

There is limited evidence for changes in serotonin (5-HT) functioning in normal aging, in part studies may imply that the number of 5-HT receptors is reduced and 5-HT turnover is increased (McEntee \& Crook, 1991). In contrast, in AD, 5-HT function clearly is abnormal. For instance, 5-HT and its metabolite 5-HIAA are lower in certain regions of postmortem AD brain. Also, a reduction in the density of 5-HT binding sites has been reported (Altman \& Normile, 1988). Tryptophan is essential in the synthesis of serotonin. Tryptophan depletion is induced with a mixture of aminoacids devoid of tryptophan. Being the mother substance of $5-\mathrm{HT}$, as well as an essential aminoacid, tryptophan depletion will rapidly lead to a 5 -HT shortage in the brain and elsewhere (Van Praag, 1996).

\section{Cognitive effects}

Until now there are only a few studies describing the effect of tryptophan depletion on human cognitive function. In two studies an increased number of errors in a proofreading task was found (Danjou, Hamon, Lacomblez, Warot, \& all, 1990; Young, Smith, Pihl, \& Ervin, 1985). Park et al. (1994) reported an effect of tryptophan depletion in the absence of an effect on mood. They 
concluded that tryptophan depletion had relatively selective effects on cognitive function, namely impairments in learning and memory processes, while leaving performance on tests sensitive to frontal lobe dysfunction substantially unaffected. Coall et al. (1995c) found that tryptophan depletion selectively increased the speed of stimulus-incompatible responses and not of compatible responses in an attentional search task. They concluded that serotonergic depletion might disrupt the inhibitory control of behavior.

Similarly, other investigators found impaired memory retrieval after tryptophan depletion in young healthy subjects. Tryptophan depletion specifically impaired long term memory performance in subjects with and without a first degree positive family history for major depression. Delayed recall performance, recognition sensitivity and recognition reaction times were significantly impaired after tryptophan depletion relative to placebo. Short term memory, perceptual- and psychomotor functions were unchanged. On the basis of these results it was concluded that tryptophan depletion specifically impairs long-term memory formation, presumably by acute lowering of 5-HT turnover in the brain (Riedel et al., submitted). The latter results were replicated in a second study. In addition to the tryptophan depletion induced impairment of memory consolidation, performance on two tasks measuring focused attention, i.e. the Stroop task and a dichotic shadowing task, was improved after tryptophan depletion (Schmitt et al., in preparation). Precisely the same combination of effects, i.e. impaired learning performance in conjunction with improved focused attention, was reported in another study (Rowley, Van, Mortimore, \& Connell, 1997).

Drug effects

As yet, no drug studies have been performed using tryptophan depletion as a model of memory dysfunction whille simultaneously testing if proserotonergic drugs enhance memory performance. L-tryptophan, the precursor of 5-HT, and tryptophan pyrrolase inhibitor improved the mental state in two out of four demented patients (Lehmann, Persson, Walinder, \& Wallin, 1981). Another study with $3 \mathrm{~g}$ of L-tryptophan showed disappointing results in a mixed population of demented patients (Smith, Stomgren, Petersen, Williams, \& Sheldon, 1984). A placebomcontrolled study with $1 \mathrm{~g}$ of L-tryptophan reported a beneficial effect on some memory functions (the memory subscale of the Information, Memory and Concentration test but not on Object learning) but not on other cognitive tests (e.g. Digit Copying Test) behavioral, and depression scores (Bentham, 1990)

Recent animal studies have shown that selective serotonergic uptake inhibitors, such as zimelidine, alaproclate, and fluoxetine, enhance memory consolidation (Briley, 1990). However, in AD patients zimeldine (Cutler. Haxby, Kay, \& al., 1985), nor alaproctate (Dehlin, Hedenrud, Jansson, \& Nörgård, 1985) were seen to have an effect on cognitive function.

\section{Mechanisms}

Memory acquisition has also been shown to be decreased by enhanced serotonergic function through stimulation of the dorsal raphe nucleus, and 5-HT antagonists have been slown to improve this induced decreased performance on different memory tests in animals (McEntee \& Crook, 1991). Although these results may seem conflicting, the effect of an eniliancement or a decrease in memory functions of 5-HT may be dependent on the specific receptor that is stimulated by the drug. For instance, 5.HT 1 A agonists (urapidil, CM 57493, and ipsapirone), 5HT IB antagonists and 5-HT 3 antagonists may increase ACh levels, while 5-HT. 1A antagonists, IB agonists and 3 agonists decrease levels (Costall et al., 1990; Peruche \& Krieglstein, 1993). In addition, animal studies have shown that the 5-HTIA agonists ipsapirone was neuroprotective against NMDA-induced damage of the cholinergic magnocellular basall nucleus (Oosterink et al., 1997). Lastly, 5-HT 1A and 5-HT2 receptors have been associated with anxiety and depression. Hence, effects on cognitive functions may be indirect, rather than direct and may thus be involved 
in the affective disorders seen in $\mathrm{AD}$.

Discussion

The tryptophan depletion model is currently under inwestigation in our laboratory. One of its drawbacks is that some subjects do not agree physically well with the procedure and experience gastrointestinal effects (i.e. throwing up, nawsea) which renders withdrawal from the experiment necensary. Alternatively, specfic 5-HT $1 \mathrm{~A}$ antagonists or 5-HT 3 agonists could be used as models to mimis cognitive deterioration.

\subsection{Models developed from behavioral (functional) backgrounds}

\subsubsection{Sleep deprivation model}

\section{Background}

Between 25 and 40 \% of the elderly population $(65+)$ complains of sleep problems (Prinz \& Vitiello, 1993). Possibly, cognitive complaints of the elderly could be associated with a fundamental lack of sleep. partly causing mental exhaustion and an inability to compensate for the lack of resources. Sleep seems to have a restorative value for the system (Feinberg \& Floyd, 1982; Oswald, 1980). According to Mikulincer, Babkoff and Caspy (1989), resource limiting factors accumulate during the day and are active during sleep deprivation. Sleep loss is interesting as a model for cognitive aging deficits. In many aspects the cognitive impairment seen in aging is similar to the effects of sleep loss or sleep deprivation (SD), which is why seweral studies have used experimentally induced sleep deprivation as a model of age-related cognitive deficiencies.

Cognitive effects

A meta-analysis of 27 studies done between 1969 and 1983, revealed that, in general, a decrease in speed (but not accuracy) on tasks have been seen but only after more than 45 hours ( 2 nights, 2 days) of sleep deprivation (Koslowsky \& Babkoff, 1992 ). By contrast, choice reaction time (CRT) is very semsitive to both aging (Houx \& Jolles, 1993) and sleep deprivation. After one night SD, subjects showed decrements on a 5 min complex Choice RT task (Wilkinson, 1990). This effect was repeated in 3 experiments.

Memory deficits (both encoding and retrieval) in young subjects after sleep deprivation has shown a functional similarity to the performance of elderly subjects (Nilsson, Backman, \& Karlsson, 1989). Yat, effects of sleep deprivation on working memory are disputed. For instance, storage in working memory was not affected by one night SD (Linde \& Bergstrom, 1992). Also Gunter, Van der Zande, Wiethoff, Mulder, and Mulder (1987) showed that after one night SD only a general slowing of transmission was seen, since all latency and RT measures slowed down while there was no interaction with memory load.

Different effects have been found for decrements in central executive tasks. For instance, Raven Progressive Matrices was impaired by SD (Linde \& Bergstrom, 1992), while Baddeley's Logical Reasoning, (Linde \& Bergstrom, 1992; Webb \& Levy, 1984) was not. In the study by Webb and Lewy (1984) young subjects were tested after 2 nights of SD $(40-45 \mathrm{~h})$. Object use and Word nemory ( 305 letter words 1 triall recall) showed decrements, while performance on Remote Associations was not affected at al.

Drug studies

Caffeine can attenuate sleep deprivation effects (Lorist, Snel, \& Kok, 1994a). Also, amphetamine has been shown to reverse some of the cognitive dysfunction after SD for 60 hours. By contrast, in this parallel groups study, neither nicotine nor l-deprenyl were shown capable of reversal (Newhouse, Penetar, Fertig, Thorne, \& et al., 1992). Alpha-methyl-para-tyrosine (AMPT) can inhibit the synthesis of catecholamine. A study by McCann et al (1992) showed that AMPT 
induced greater sleepiness when given during sleep deprivation and also caused impairment on a variety of cognitive tests. Since earlier amphetamine (catecholamine simulant) has been shown to improve performance after sleep deprivation, the cognitive decline after sleep deprivation may be associated with declined levels of catecholamines.

\section{Mechanisms}

During sleep loss most clearly vigillance performance falls while sleepiness and fatigue increase (Bonnet, 1986; Bonnet, 1987; Carskadon \& Dement, 1985; Johnson, 1982; Webb, 1985; Webb \& Levy, 1982; Webb \& Levy, 1984). Yet, wigilance tasks are not interesting in modelling aging effects, since vigilance has not shown to be primarily affected in aging (o.g. only in the 1 ast 15 mim of a 60 min task age effects are seen: Kausler, 1991). However, when vigilance tasks are high work paced, already after 2-3 min performance has been shown to be decreased after one night of SD (Smith \& Maben, 1993). Pacing tasks puts at high demand upon perceptual processing.

Similarly to scopolamine and hypoxia, effects on stimulus evaluation have been noted to occur after sleep deprivation (Lorist, Snel, Kok, Mulder, 1994b). For instance, a decrease in perceptual sensitivity, as measured with a signal degradation test, has been shown to occur after sleep deprivation (Steyvers, \& Gaillard, 1993). In a study done by Hockey (in: Kjellbere, 1977), a decrease in sensitivity for peripheral stimuli was seen after 10 min. In this dual task, young subjects had to do a tracking task and detect central and peripheral light signals simultaneously. According to the author, these findings were caused by the narrowing of the attentional ability. By contrast, according to Niatoh (1976) these effect are due to the slowing of eye movements (more difficulties in eye positioning). Similar discussions are seen in aging research (Kausler, 1991). However, according to Lorist, Snel, Kok, Mulder (1994b) the decrease in P300 amplitude after sleep deprivation indicates that the allocation of available resources subserving perceptual processing is reduced. Fatigue hence affects the encoding stage of information processing while central memory processes remain unaffected. Caffeine was seen to reverse these effects in this study.

\section{Discussion}

Conflicting results have been found in sleep deprivation studies (Morris, So, Lee, Lash, \& Becker, 1992). Baseline differences on complex tasks may have clouded effects in some studies (Bonnet, 1989; Webb, 1985; Webb \& Levy, 1982). Furthermore, in the studies using elder subjects, especially in middle aged (40-49 yrs) and elderly subjects $(50-60 \mathrm{yrs})$ large decrements are seen after two nights of sleep deprivation on tasks like the Object use, Anagrams, Word detection and Visual Search tasks (Webb. 1985; Webb \& Levy, 1982), while young subjects in these studies were only affected on the vigilance tasks. This may indicate that differences found in studies could be due to the possibility of compensation by investing additional effort. The possibility to compensate for decreased mental resources with additional effort can be seen especially in younger subjects (Johnson, 1982; Minors \& Waterhouse, 1981; Morris et al., 1992; Webb \& Levy, 1984). Effort can be experimentally manipulated with feedback. In a study done by Steyvers and Gaillard (1993) young subjects showed to be able to overcome sleep deprivation effects (one night, testing at noon) with knowledge of results and reward on a signal degradation task by mobilizing additional effort. The choice to invest more effort can be influenced by nonspecific factors, such as motivational or instructional factors. P300 is a positive potential in EEG seen after $300 \mathrm{~ms}$ in response to a target or probe. P300 is consideres to be a reliable index for the avallability of processing resources (Veldman, 1992).

Furthermore, most cognitive effects are onlly seen after two nights of SD, which is quite cumbersome for experimenters as well. This decreases the practical value of this model. However, P300 also seems a good early marker of sleep deprivation effects (Morris et al., 1992). Their study 
with 15 healthy volunteers after only 18 hours of $\mathrm{SD}$ alleady revealed a significant decrease in P300 amplitude and an increase in P300 latency. These cluanges correlated with body temperature and fatigue. Reaction time in this study did not show a significant change. Changes in P300 (decrease in P3 amplitude and an increase in P3 latency) have also been noted in normal aging (Picton, Stuss, Champagne, \& Nelson, 1984). Hence, the sleep deprivation model may be of rellevance when the P3O0 paradigm is used.

\subsubsection{Model of maximum physical excercise}

\section{Background}

The underlying theory to this model is the effect of prolonged, endurance exercise on information processing capacity or mental resources. Fatigue or a reduction in mental and physical resources has been hypothesized to underlie the negative influence of intense exercise on cognition (Tomporowski, Ellis, Stephens, 1987). Conversely it is questioned whether physical performance decrements over time, e.g. in endurance exercise performance, are affected by mental fatigue as indexed by cognitive performance decrements, rather than by pure physical fatigue, as indexed by decteased muscle strength.

\section{Cognitive effects}

Several studies have described a negative effect of strenuous physical exercise on performance of cognitive tasks (Gutin, 1973; Hancock \& McNaughton, 1986; Isaacs \& PohIman, 1991; McMorris \& Keen, 1994; Salmela \& Ndoye, 1986). However, not all authors have found strenuous exercise to have a negative effect on cognitive functions (Adam, Teeken, Ypelaar, \& Verstappen, in press; Bard \& Fleury, 1978; Cote, Silmela, \& Papathanasopoulu, 1992; Fleury, Bard, Jobin, \& Carriere, 1981; Hogervorst, Riedel, Jeukendrup, \& Jolles, 1996; Paas \& Adam, 1991; Tomporowski et all., 1987).

The various results are difficult to compare. Studies used subjects from different backgrounds and with different levels of fitness, and different cognitive tasks were used either during or after exercise of a varying duration (McMorris \& Keen, 1994; Tomporowski \& Ellis, 1986). Often, exercise of a short duration (varying from 2 to 15 min) was used and the level of physical and. mental fatigue was not ewaluated. Matters are further complicated since as Tomporowski and Ellis (1986) stated there is no agreement on the definition of fatigue. It is thus unclear whether and how physical fatigue affects simple and complex cognitive processing, and what relation, if any, exists between physical and mental fatigue.

\section{Drug studies}

Only one study by Hogervorst, Riedel, Kovacs, Brouns, \& Jolles (submitted) has been done using strentous physical exercise as a model to test a putative cognition enhancer. In this study, low and moderate dosages of caffeine were seen to affect speed and accuracy of cognitive functions positively after exercise. To our current knowledge no other potential cognition enthancing drug has been studied in the model of physical exhaustion.

Mechanisms

According to a recent review by Davis and Bailey (1997), the central nerwous system is likely to play an important tole in mediating subjectively experienced fatigue, since changes in motivational levels have profound effects on endurance performance. Further, the first indication that fatigue is imminent, is an increased perception of effort necessary to exert a desired force or power output. This is later followed by a failure to keep up the required output that is not explained by a dysfunction in the muscle itself. Both animal and human studies suggest that an increase in central serotonergic activity or a (coupled) decrease in dopaminergic and / or cholinergic activity during exercise may be responsible for mental fatigue occurring. Also, it is known that brain centers 
involved in complex mental processing have a higher metabolic demand (Home, 1992) and hence would be expected to show decreased functioning when the overall energy level is low, dne to restorative actions in the muscles after exhaustive physical exercise. Furthemore, oognitive performance during, or immediately after prolonged physical exercise may be (relatively) impaired by (brain) hypoxemia due to a reduction in arterial oxygenation during exercise at higl work rates (Powers, Martin, \& Dodd, 1993).

Discussion

Drugs that enhance cerebral metabolism could be used in this model. The model can be carried out with, either prolongued physical exercise, or until subjects have rached a level of maximum exhaustion. Young subjects, especially thoroughly trained athletes, often show the possibilities of compensation through investing additional effort for cognitive processing (Hogervorst et al., 1996). Hence, possibly middle-aged healthy subjects should be used instead of young subjects. Still, caffeine in the model using young athletes was seen to enhance cognitive functions on top of the effect of exercise (Hogervorst et al, submitted).

\subsection{Putative new models}

In this paragraph a few putative new models are described. For instance, the deficient glucose metabolism seen in aging and dementia could be modelled by inducing intracellular hypoglycemia to investigate the effect of possible cognition enhancers that are thought to act upon brain metabolism. Also, briefly the possibility of the use of catecholamines as models for age-related cognitive deficiency is described. Lastly, an alternative model for sleep deprivation is given.

\subsubsection{Putative models for cerebral metabolism}

\section{Background}

A deficient glucose metabolism seems to play an important role in age-related cognitive dysfunctioning (Smith, 1984) and dementia (Hoyer, 1992). In aging, blood glucose levels can be paradoxically increased in plasma. Yet, due to an increased insensitivity in the insulin receptor, a hypoglycemia occurs intracellular.

The brain is specifically sensitive to hypoglycemia. During severe hypoglycemia, for instance, cellular oxidation is observed (Vannucei \& Mujsce, 1992). Chronic hypoglycemiat can oasuse a reversible demential state and can be inflicted by $\mathrm{MAO}$-inhibitors, salicylates or high insulin levels (Van der Meer \& VanTilburg. 1983).

\section{Cognitive effects}

Research has shown that when insulin-dependent diabetic patients were assessed during an ancute severe insulin-induced hypoglycemia, there was a significant performance decrement on 5 out of 7 neuropsychological tests (even though there was a conterregulation with GH, glucagon and cortisol). No decrement was seen on tasks that demanded visual motor coordination. However, especially motor speed (digit symbol, reaction time, aiming center, line tracing time and errors) declined. Patients were not always aware of their actual metabolic state.

Although some of the results may be associated with trembling (side effect) probably most can be attributed to low levels of glucose in the brain, since the motor cortex is very vulnerable to glucose deprivation (Lingerfelser et al., 1992).

Drug studies

Extensive evidence indicates that relatively modest increases in circulating glucose enhances 
Leaming and unemory processes in rodents and humans (Gold, Deary, Macleod. Thompson, \& Frier, 1995). Cogintion enhancers, amed at improwing the uptake of glucose and metabolism in the brain may be helpful in counteracting age-related cognitive decline.

Discussion.

A hypoglycemic state could be induced in young, healthy subjects through exhaustive physical exercise without glucose substitutes. Another method of inducing hypoglycemia could be through administration of 2-deoxyglucose, which mimics the intracellular hypoglycemia and the extracellular hyperglycemia $(147.2 \mathrm{ng} / \mathrm{dcl})$ seen in aging. This method has been used as a challenge in schizophrenic patients and normal vollunteers and is well tolerated (Breier \& Buchanan, 1992: Breier, Davis, Buchanan, Moricle, \& Munson, 1993).

\subsubsection{Possible catecholamine models}

\section{Background}

A review by McEntee \& Crook (1990) describes that substantive evidence indicates a decline in brain catecholamines in brain activity in aging and dementia. Ollat (1992) argued that amongst the various disturbances of neurotransmission accompanying cerebral aging, the dopaminergic (DA) insufficiency is the most constant, the earliest and the most severe. The loss of the dopaminergic innervation of the neostriatum is a prominent age-related change, which corresponds with the age-related loss of dopaminergic cell bodies from the substantia nigra (Palmer \& DeKosky, 1993). Although some controversies remain, in $\mathrm{AD}$, decreased dopamine levels and decreased numbers of D2 receptors have been detected (Gottfries et al., 1983; Gottries, Bartfai, Carlsson, Eckermas, \& Svennerholm, 1986).

In animal studies, in which dopaminergic neurons were destructed (with 6-OH-dopamine), administration of haloperidol, a dopaminergic antagonist, or blockade of catecholamine synthesis with alpha-methyl-paratyrosine (AMPT) an impaired memory performance became evident. The latter two effects could be reversed with dopaminergic agonists (Briley, 1990). Halloperidol has been used in a few studies with healthy volunteers, whereas AMPT has only been used in humans as a challenge procedure in antidepressant and sleep research (Delgado et al., 1993; McCann et al., 1992).

Also, in studies of a subpopulation of $\mathrm{AD}$, a substantial cell loss of the locus caeruleus has been reported. The locus cereleus is the primary source of norepinephrine (McEntee Crook, 1990).

Clonidine, a mixed noradrenergic alpha $1 /$ alpha 2 agonist, has been seen to dose-dependently decrease plasma noradrenaline levels selectively and to reduce mental performance scores in both anmals and hurnans (Kugler, Seus, Krauskopf, Brecht, \& Raschig, 1980) Experimental studies with haloperidol, AMPT, or clonidine deserve the attention as possible pharmacological models for age-related cognitive dysfunction.

Cognitive effects

Brain catecholamines can be rapidly reduced by inhibiting their synthesis with atpha-methyl-paratyrosine (AMPT). This method has been used as a challenge in depressed patients (Delgado et al., 1993) and healthy subjects to investigate the role of brain monoamine function in the pathophysiology of depression and the mechanism of action of antidepressant drugs. Treatment with AMPT or treatment with sleep depriwation increased sleepiness without producing marked or consistent deterioration in performance. By contrast, subjects who received both treatments reported greater sleepiness than those receiving either treatment alone, and developed severe cognitive impairment on a variety of tasks (McCann et al., 1992).

A study by Coull, Sahakian, Middleton, Young, Park, MeShane, Cowen, and Robbins (1995c) in young, healthy volunteers found that haloperidol generally slowed reaction times during 
attentional search. Also Rammsayer (1989) found that halloperidol impared RT in normal volunteers. Effects of dopamine on memory may thus be indireet through its effects on attention and arousal functions. According to Goldman-Rakic (1996) accumulating evidence indicates that dopamine has a major role in regulating the excitability upon which the working memory function of the prefrontal cortex depends. Others conld not detect an effect of haloperidol on memory and other psychometric functions, save for the critical flicker fusion test (CFF) (Hindmarch \& Tiplady, 1994). Some even found an improvement of haloperidol on choice and simple visual RT in healthy volunteers (King \& Henry, 1992).

In humans it is suspected that norepinephrine mediates various attentional processes, such as selective attention, and that it affects memory functions indirectly (Coull, Middleton, Robbins, \& Sahakian, 1995a). For instance, norepinephrine may help allert the forebrain in processing important external stimuli, prevent distraction by irrelevant stimuli, and counteract effects of stress on task performance (Coull, 1994). In a study by Coull, Middlleton, Robbins \& Sahakian (1995a) the low dosages of 1.5 and $2.5 \mathrm{microgram} / \mathrm{kg}$ clonidine, were reported to impair sustained attention. In another study by Coull et al (1995b), the low dosages of clonidine (1.5 and 2.5 microg/kg) were seen to increase the impulsivity in responding on a working memory task. This was, according to the authors, not because of an executive, but because of a mnemonic dysfunction. Frith et al. (1984) found that clonidine in a low dose impaired paired-associate learning. However, no effects were seen on digit span, immediate or delayed recall of paragraphs.

Drug studies

Some studies suggest that MAO-B inhibitors, which enhance dopaminergic levels, can slow progression in $\mathrm{AD}$ and Parkinson's disease. $\mathrm{MAO}-\mathrm{B}$ seems elevated in postmortem $\mathrm{AD}$ brains (Tariot et al., 1987). L-deprenyl, a MAO-B inhibitor, used in a controlled trial, lead to improvement in cognitive function of AD patients (Piccini et at. 1990 in: Pathy, 1993). In another study, $17 \mathrm{AD}$ patients were assessed in a double blind, randomized design. L-deprenyl $(10 \mathrm{mg} / \mathrm{d})$ improved performance on a selectiwe reminding task and free recall. L-deprenyl, however "did not affect episodic learning and memory, category retrieval, knowledge, attention, and vigilance (Tariot et al., 1987).

In healthy volunteers it was demonstrated that administration of Levodopa (L-DOPA) improves memory performance (Murphy \& Henry, 1972). L-DOPA combined with benserazide, a peripheral active carboxylase inhibitor, showed some improvement in 14 patients with $A D$ on cognitive tests over a 24 weeks period (Jellinger, Flament, Riederer, Sclimid, \& Ambrozzi, 1980 ). Again, two placebo controlled studies did not show a positive effect of $L$ DOPA in AD patients (Kristensen, Olsen, \& Theilgard, 1977; Schneck, 1983). There is little evidence that L-DOPA given alone improves cognition in AD.

Clonidine may act as a postsynaptic noradrenergic agonist in higher dosages. For instance, the study by Coull, Sahakian, Middleton. Young, Park, McShane, Cowen, and Robbins (1995c) found that clonidine in a higher dosage acted to broaden the focus of attention in young healthy volunteers. However, a beneficial effect of clonidine in AD patients has not been detected (Mohr, Schllegel, Fabbrini, \& al, 1989). Coull (1994) concluded that alpha 2 -adrenoreceptor agonists, such as clonidine but also guanafacine, have little therapeutic value in patients with dementia of the Alzheimer"s type but may have clinical use in patients with frontal lobe symptomatology, such as patients with Korsakoff disease, attention deficit disorder, or schizophreniat.

Mechanism.s

According to Briley (1990) possible improvement of cognitive function in AD patients with \|-depreny! would be mediated by increased arousal, since especially surstained attention seemed to be improved. Again, the level of alertness at the time of learning, however, should not be dismissed 
as a non important effect since it is crucial to a large degree on what is remembered and how much is remembered (Squire, 1987). However, some authors have shown that a dopaminergic depletion (e.g. in Parkinson) is also associated with major depressive episodes, which may induce cognitive deficits that are not direculy related to aging (Mann. Kapur, Schatzberg, Schwartz, \& Willner, 1995).

Ollat (1992) states that the cognitive symptoms seen in aging (difficulty with complex tasks under time pressure) are analogues to those of frontal symptomatology. As such, these symptoms reflect a de-afferentation of the frontal lobe, explained through a deprivation of effective dopaminergic innervation. Also complex interactions exist between dopaminergic and cholinergic systems (Levin, McGurk, Rose, \& Butcher, 1990). Similarly, in animal studies, when norepinephrine was depleted in the forebrain, naloxone induced memory facilitation was decreased and scopolamine induced impairments were increased. The latter finding suggest that noradrenergic systems may affect memory partly through cholinergic systems (Chen, Chiu, \& Lee, 1992; Pathy, 1993). As such, possibly combination therapies could be of value.

Discussion

Both haloperidol and clonidine have shown effects on cognutive functions in young subjects. These effects are not easily interpreted as to whether they result from direct or indirect mechanisms. An alternative would be to model nonspecifically the catecholaminergic deficit, by means of its synthesis inhibition, with AMPT. However, due to AMPT"s toxicity, a better method would be catecholaminergic depletion by means of the depletion of tyrosine and phenylalanine. This can be accomplished through the administration of a balanced amino acid mixture lacking tyrosine and phenylalanine. It has been demonstrated that this method is physiologically active, in at comparable fashion as tryptophan depletion (Moja, Lucini, Benedetti, \& Lucca, 1996). This method has not been used in conjunction with assessment of cognitive functions. It deserves attention, possibly in combination with other models such as sleep deprivation or scopolamine, to induce memory and/or vigilance deficits. The model would enable the evaluation whether catecholamines explain additionally some of the age-related cognitive deficiencies, which are not explained by the scopolamine alone, such as certain attentional, planning and executive functions.

\subsubsection{An alternative model for sleep deprivation}

\section{Background}

The most striking change in the sleep pattern of the elder population is the repeated and frequent intertuption of sleep and an increase in the time spent awake (Webb \& Campbell, 1980). A deficiency in certain sleep stages has been thought responsible for the reduction in cognitive capacity or resources in aging. In humans Slow Wave Sleep (SWS, delta EEG activity in stage 3 and 4) declines with age (Home, 1992). SWS has also shown to decline with the programmed cell death and the alternations in cortical symaptic density in the second decade and may reflect a recuperative process for the brain to reverse the effects of wear and tear (Feinberg \& Floyd, 1982). There is some evidence that selective sleep deprivation (i.e. the internuption of particulat sleep stages) may be relevant as a model for age-related cognitive deficiency.

Cognirive effects

Several experiments suggest that random sleep disruption results in a lowered sleep quality and impaired cognitive functioning (Bonnet, 1986; Bonnet, 1989). According to Bonnet (1986) sleep disruption causes similar effects as sleep deprivation, causing increased sleepiness decreased performance ability and degraded mood. In one study (Bonnet, 1986) four sleep schedules were examined in 8 young subjects: a 64 hour total sleep loss; disruption after each min of sleep; 10 min of sleep before disruption; and 2.5 hours of sleep before disruption. Immediately after 
awakening subjects performed 30 min of Wilkinson addition (atcuracy) and: 30 min of Wilkingon Vigilance (hit rate). Also the Clyde Mood Scale and a reaction time task was performed after a sleep latency test. Results indicated that sleep continuity in excess of 10 min whithout arousal seems to be restorative rather than specific stages (SWS or REM) or total sleep time. Furthermore subjects have shown not to be aware of sleep disruption, hence this may eliminate the intervening effect of effort as is seen in sleep deprivation.

Drug studies

Melatonin, the main mediator of circadian mechanisms associated with the organization of sleep. may be the main effective mediator in increasing steep quality and cognitive function in mood disorders, aging, and dementia (Gross \& Gysin, 1996; Maurizi, 1990; Myers \& Badia, 1995). Sleep initiation and maintenance were improved by melatonin in elderly insomniacs (Haimov et al.. 1995). Again, some investigators found that only half of the insomniacs $(n=15)$ profited to a minor extent from melatonin and concluded that melatonin is not of clinical value in treating psychophysiological insomnia (Ellis, Lemmens, \& Parkes, 1996). Another study found that melatonin improved sleep quality after night shifts of nomal healthy subjects. However, melatonin had no effect on visual search speed and accuracy, while it was seen to negatiwely affect the speed of memory scanning (Folkard, Arendt, \& Clark, 1993).

However, morning light therapy was seen to increase total and nocturnal sleep time and reduced the behavior disorders in demented patients, possibly through synchronizing melatonin secretions (Mishima et al., 1994).

\section{Mechanisms}

Since melatonin can also act as an antioxidant, interesting in this respect are the findings that in the elderly, effects of oxidative stress may indeed be an important factor in the atrophy of neurons and may be related to sleep quality. During NREM the brain shows a $30 \%$ reduction of the glucose metabolic rate as compared to wakening states (Horne, 1992). A lowered metabolism results in a lowered production of free radicals and NREM sleep could then as such temporarily protect the brain against a build up of free radicals. Possibly, during this period compensational mechanisms against oxidative damage could take place.

\section{Discussion}

Following logically from these findings, cognitive enhancement in the elderly should focus on increasing the SWS period. Studies have shown that SWS increases when subjects are shown novel and interesting scenes. Especially elderly subjects often do not engage in novel situations, which may be beneficial (Horne, 1992). Furthermore, SWS has also been known to decrease with stress. anxiety, insomnia, and depression (Home, 1992). These factors, which often occur in the elderly, should be taken into account.

\subsection{Discussion}

The most ubiquitously used models as well as some new and promising models for age-related cognitive deficiency and dementia have been reviewed. In this final paragraph, the general problems encountered in trying to model cognitive impaiments are summarized.

\subsubsection{Subjects}

In the models described usually young healthy subjects are used to test cognitive enhancers. The aim, in using a model to test putative cognition enhancers, is to model one age-associated detrimental factor in thealthy subjects to induce a state of diminished cognitive functioning. Yett, young subjects often have great capacity to compensate and have been reported to overcome 
induced states. This was seen to occur in thypoxia (Bahrke \& Shukitt, 1993), hyperventilation (Balke \& Lilehei, 1956) steep deprivation (Johnson, 1982; Minors \& Waterhouse, 1981; Morris et al., 1992. Webb \& Levy, 1984) and after stremuous physical exercise (Hogervorst et al., 1996). Further, if effects induced do not subside ar can be easily compensated for, chances are that inteversible damage may be unduced. However, already in the fourth and fifth decade of life, a decrement of cognitive functions can be detected (Jolles et al., 1995b) and middle-aged, healthy subjects may be a better alternative than younger subjects (Hogervorst, Riedel, Schmitt, \&olles, submitted).

In addition, it is debatable whether results obtained through studies with young healthy subjects, can be extrapolated to elderly subjects. The elderly differ with the first group on multiple factors, including the altered pharmacokinetics and pharmacodynamic responses to some drugs (e.g. benzodiazepines) as weil as inany environmental factors (social input, exercise, exposure to light). This is especially true for pathological conditions such as AD subjects.

Further, positive results in young subjects with cognition enhancers may not always be indicative of a therapeutic effect in Alzheimer"s patients. In fact, some authors conclude (Gamzu et al., 1990) that trials with normals can only define the range of doses that affect the CNS or possibly define the duration of action. They base this notion on the problems generated in the extrapolation of highly sophisticated neuropsychological thest data of a young, optimally functioning student population to the everyday life experiences of a demented population. By contrast, in seeking treatment for AACD patients, using young or middle-aged heaithy subjects with an experimental model can be considered an adequate method to gain insight in factors important in pathogenesis and treatment.

\subsubsection{Measurements}

The wide variety of tests used makes it wery difficult to compare results of studies. Also many of the tests used have a disputed validity, in lacking the sensitivity to detect milder forms of (brain) dysfunctioning (Bergener, 1993). Other tests measure such obscure functions that their practical relevance is almost nil. Further, self-report questionnaires of improvement, which have been used in several studies, have shown not to be very reliable measures of the actual level of performance (Huppert, 1991).

A second problem which has to be noted is the use of testbatteries consisting of a variety of tests. This practice increases the probability that only a few tests of the battery will show positive outcomes and the other (neutral) outcomes accordingly are not mentioned. Gamzu. Birklhimer, Hoover and Gracon (1990) warn for the nse of multiple outcome measures without statistical compensation (Bonferroni, e.g.) for multiple analyses. Furthermore, as for more complex tasks, repeated trials often induce learning effects which may overrule the small effects of cognition enhancers. For this reason in clinical trials of subchronic treatment with cognition enhancers, measures should always be taken to rule out learning effects; i.e. the implementation of practice sessions, the use of parallel versions of the assessments instmments as well as order balancing of parallel versions of the assessment instrument over repeated assessments. However, it is difficult to understand that research is published not fulfilling these long known basic methodological rules of experimental design (Winer, 1971).

The choice of tests should be dependent on the population that is the ultimate target of treatment. However most studies show that the decline induced by models may be comparable to normal aging (e.g. scopolamine and hypoxiaj but does not equal the decrement seen in Alzheimer's dementia (Hogervorst et al., submitted; Riedel, \& Jolles, 1996). Experimental drug research in non demented subjects up to now has been less popular than drug research in patients with Alzheimer*s 
Dementia (Jolles et al., 1995b). As was defined earlier by Black ford and LaRue, in Age-Consistent Memory Impaiment (ACMT), which is similar to the concept of ARCD, performance should be within ISD of the mean established for age on $75 \%$ or more of the tests administered. For Late Life Forgetfuness performance should be between 1 and 2 SD below the mean established for age on $50 \%$ or more of the tests administered. Hence, the cognitive deficiencies in ARCD or ACMI can be modelled in young subjects with the current models.

With regard to neurophysiological tests, normalization of slowed EEG, an increase in cortical evoked potential (P300 amplitude and shortened latency) and increase in peripheral nerve conduction times have been reported after cognitive enhancers. Other authors do not believe these tests to be very sensitive (Hollister, 1990). According to Delacour, Bassant, Onofyj, Santucei, and Kleinlogel (1990), however, there is a considerable body of evidence that both in animal and human research cognition enhancers modify the parameters of the EEG power spectrum, characterized as EEG-activating. Nootropic drugs all induce an increase in fast frequency (beta) activity in humans. A limitation of this approach is that the role of nonspecific effects of changes in arousal are difficult to determine. This need not be an obstacle since one mode of action of cognitive enhancers might be their property to promote optimal levels of arousal or to facilitate sustained attention. Further, this limitation can be bypassed by using recordings from large populations of neurons, the evoked potentials (ERP) techniques, such as the P300. Changes in P300 (decrease in P3 amplitude and an increase in P3 latency) have been noted in normal aging and $\mathrm{AD}$. In fact, a sensitivity and specificity of $60 \%$ has been seen in most studies and nake $\mathrm{P} 3 \mathrm{a}$ potential clinical marker for AD. Sleep deprivation, acute ethanol and nitrous oxide exposure have also been seen to increase P3 latency and decrease the amplitude in a dose dependent matter. This could indicate that these experimental inductions may be potential models for age-related normal and pathological cognitive deficiencies.

\subsubsection{Models}

The best model would probably be a model which allows for the testing of drugs in young subjects but which can also be used as a challenge procedure in elderly healthy as well as AACD, and AD patients The scopolamine model is an example of such a model. Further, the scopolamine model in general seems to have an adequate therapeutic predictive validity. To further enhance this validity, combination models could be used (scopolamine in combination with dopamine or norepinephrine blockers e.g.). The benzodiazepine model is not considered to be a good model for age-related cognitive decline since it offers little to no predictive vallue.

Several models concened with cerebral metabolism have also been described. Hypoxia is already used as a model, although the specificity of this model may be questioned. Also, complex instruments are needed (e.g. a hypobaric room) and cardiac side-effects (syncope) should carefully be monitored. The hyperventilation model does not seem a good alternative, since it is highly unspecific and, for instance, the side-effects of overbreathing have not been extensively studied. Yet, overbreathing itself was seen to induce a strong slowing of response times and may be used as a model for the neural noise which according to some authors is an important factor in aging. Hypoglycemia may offer possibilities in minicking the decrease in cerebral metabolism. However, the risk for shock and irreversible cell damage should be taken into account. Also, motor side-effects (trembling) and possibly unjustified inductive reasoning can render extrapolation to the aged difficult (e.g. perhaps a decrease in metabolism is the result of a lowered demand of systems due to lowered activity). Also, a variety of behavioral models has been described. The capacity models (sleep-interruption and maximum physical exhaustion) may offer new possibilities in testing cogritive enhancers. 


\subsubsection{Design and statistics}

Many studies have reported degree of improwement from placebo as compared to baseline, which is a nonspecific response based on the attention given to patients during a study. As this effect seems unavoidable, the effect of memory training could be compared with drug trials to see what the merits are of using training instead of drugs (Hollister, 1990). Although little attention has been. given to the combination of both, Deberdt (1994) described studies by Israel who compared gingko biloba and piracetam with memory training in non demented subjects complaining of memory problems. The effects of drugs and memory training were seen to be complementary. Some cognitive functions were seen to benefut from both treatments (attention and perception in gingko biloba and leaning in piracetam) which indicated a mutually potentietting effect of drug treatments and memory training.

Most studies that tested patients have used double blind parallel groups designs. Changes reported are usually small. There is no indication that even if a p-value reaches significance and the percentage of improvement is in the range from $10-20 \%$, that this degree of improwement is to be expected among all treated patients. A small number of a subgroup of patients may show meaningful improvement but the majority of patients can remain unchanged. To overcome the possible infuence of unknown personal variables, time-series destgns could be used in which a patient is his own control. However, these designs are time-consuming since they are longitudinal Furthermore, particularly this kind of research is vulnerable for improper inductive interferences and causal reasoning. Cause and effect should be carefully considered even though relations might seem very sensible and functional. Many aspects seen in aging or as a result of drug administration could rather be relatively minor important side-effects. The distinction between etiology and pathogenesis (and treatment) should be kept in mind. Etiology research needs to focus on many different aspects, while treatment should focus on those points that optimize and activate resources, supplement deficiencies and halt the decomposition of systems.

\subsection{Conclusions}

For the majority of the population cognitive aging evolwes as a normal process. Allain, Boyer, Kossman, Lepine, \& Kanowski (1990) have stated that normal aging should not be a target in the development of cognition enhancing drugs. Yet, because the average age increases and there is a higher demand of the consumer to function optimally for a longer period of life in which the slowing of functions is thot felt as desirable, the need for cognitive entancers in the population can be expected to show an increase. This demand may even be made by young subjects in the future, as is already seen in the increase in the use of smart drugs.

Up to now, the main focus in the development of cognitive enhancers has been on halting or reversing the effects of $A D$. $A D$ is a highly disabling and dehumanizing disease that shows a steady increase in prevalence due to the increase in the percentages of very old subjects in western society. This increase in prevalence also increases the pressure on the national budget and hence justifies research into cognitive enhancers. For this group great advances in diagnostics have been made in the last decade which enables a better evaluation of drug effects (Jolles et al., 1995b). However, more research into the etiology, pathogenesis, diagnostics and treatment of the borderland group of patients, the so called AACD group, still needs to be carried out. Models of cognitive deficiency may be of particular value for this group. 


\title{
Chapter 3
}

\section{The Effects of Age, Health, and Speed of Information Processing on Performance of a Word-learning test with Distraction.}

Eef Hogerworst, Wim J. Riedel, Peter I. Houx ,Jeroen A. J. Schmilt, \& Jelle Jolles

\begin{abstract}
We investigated the effect of age on performance of a complex word-leaning test with and without distraction. Four age groups ( $n=111,24$ to 77 years of age) were included. We atso assessed the mediating effect of education, health, and speed of processing on memory performance by using regression analyses. Interactions were seen between age and learning and between age and rate of forgetting for the word-learning test with distraction but not for the word-learning test without distraction. Slowness and a decrease in subjective health explained this age-related variance in performance of both memory tests.
\end{abstract}

\section{Intraduction}

Nearly all cognitive functions decrease with age. For instance, most cognitive processes become slower (Jolles, 1986; Poon, 1993). However, some forms of learning and memory, such as short-term memory, semantic memory, and recognition, remain relatively stable with age, while other forms, such as free recall, detriorate with age (Craik \& Bird, 1982; Earles et al., 1997; Park et al., 1996; Woodruff-Pak \& Finkbiner, 1995). There are competing theories about the mechanisms underlying these differences (see: Light, 1991). According to some authors, the age-related memory impaiment is greater for complex tasks which require processing resources, such as working memory capacity or attentional control (Cerella, Poon, \& Willams, 1980; Craik \& Bird, 1982; Hasher \& Zacks, 1979; Salthouse, 1985; Salthouse \& Coon, 1993). Others, however, question the 'complexity' hypothesis, since the age-related decrease in performance of several more complex memory tasks (e.g. "digit span backward"), is not always greater than that for less complex tasks, e.g. 'digit span forward' (Verhaegen, Marcoen, \& Goossens, 1993). Task "complexity" may thus be too broad a concept to account for the age-related differences in memory performance.

The literature suggests that there is an age-related vulnerability to distraction in a variety of tasks (McDowd \& Birren, 1990), especially if the distraction task involves meaningful material (Carlson, Hasher, Connely, \& Zacks, 1995). Also, in everyday life situations people are often distracted when rehearsing or learning new information. It has been hypothesized that elderly people may be more susceptible to distraction because they have a diminished ability to inhilbit irrelevant information from entering working memory (Gerard, Zacks, Hasher, \& Radwansky, 1991).

We investigated the possible age-related difference in memory performance on a word-learning test (Brand \& Jolles, 1985) as compared to memory performance on a more complex 
word-learning test which required inhibition of distracting meaningful information. The distraction consisted of a simultaneously presented auditory word list which had to be ignored during presentation of the visual targets. Most studies into the effect of age on memory functions only compared young (on average 20 years of age) and older adults $( \pm 70)$, but relatively little is known about the relationship between age and memory across the life span (Nilsson et al., 1997). Because cognitive performance has already been found to be diminished in the fourth and fifth decades of life (Houx, Vreeling, \& Jolles, 1991; Jolles, Houx, van Boxtel, \& Ponds, 1995a), we included two middle-aged groups.

We also inwestigated whether certain demographic factors are associated with the age-related wariance in performance of the two word learning tests. Education and health factors are thought to influence cognitive performance in the elderly people (Huppert, 1991; Salmasso, 1993). Indeed, both objective health factors. (Houx \& Jolles, 1993; Houx \& Jolles, 1994) and subjective health have been reported to be related to a decrease in memory performance (Perlmutter \& Nyquist, 1990). However, other investigators did not find that controlling for subjective health attenuated the age-related variance in performance (Earles et al., 1997; Nilsson et al., 1997; Satthouse, Kauster, \& Saults, 1990).

It is possible that the common denominator of the effect of age, education, and health on memory performance is the slowing of cognitive functions. Processes that are conducted slowly are not as effective as those conducted more rapidly (Bryan \& Luszcz, 1996). For instance, memory consolidation functions may be largely dependent on the speed with which information is activated and processed in working memory (Baddeley, 1986; Salthouse \& Babcock, 1991). Several studies have found that the speed of information processing is a fundamental factor in explaining the variance in the age-related decline in memory functions (Bryan \& Luszcz, 1996; Fisk \& Warr, 1996; Park et al., 1996; Salthouse \& Babcock, 1991; Salthouse \& Coon, 1993). Furthermore, Earles, Tabor-Connor, Park, Smith, and Zwahr (1997) showed that the speed of information processing mediated nearly all the age-related variance in inhibition and susceptibility to interference.

Thus, because age-related memory impaiment is believed to be more pronounced in complex tasks and because the performance of aging subjects may be more disrupted by distraction, we hypothesized (1) that there would be a greater age-related difference both in learning and in the rate of forgetting items of the word-letrning lest with distraction than in the test without distraction: (2) that the level of education and objective, but not subjective, health would explain the age-related variance in performance of both learning testss to a greater extent than the factor age itself; and (3) that the speed of information processing would explain more of the age-related wariance in performance of the complex word-learning test than it would the variance in the word-learning test without distraction and that it would overrule the effects of age, the level of education, and the health factors in the regression analyses.

\section{Method}

\section{Participants}

One hunderd eleven subjects (aged 24 to 77 years) were randomly drawn from the Registration Network of Family Practices (RNH) as part of the Maastricht Aging Study (MAAS, panel study A3: Jolles, Houx, van Boxtel, \& Ponds 1995). Exclusion criteria were medical conditions which are thought to interfere with nomal cognitive function, such as cerebrovascular pathology, brain surgery, tumors of the nervous system, congenital malformations of the nervous system, multiple sclerosis, parkinsonism, epilepsy, dementia, organic or affective psychosis, schizophrenia, 
electroconvulsive therapy, the regular use of psychotropic drugs, mental refardation, and hemodialysis for renal failure. Volunteers were paid for their participation and were treated in accordance with the "Ethical Principles of Psychologists and Code of Conduce" American. Psychollogical Association, 1994).

The participants were stratified by age, sex, and the level of occupational achievennent (LOA). Four discrete age classes were clustered, i.e. young (24-36 years of age), young middle-aged (37-49 years of age), old middle-aged (50-62), and old (65-77 years of age). The LOA has a 7 -point score which ranges from " 1 " (unskilled, simple labor), to " 7 " (highly specialized labor" at an academic level) and estimates the highest level of professional activity. It is based on a detailed description of a person's occupation (Brand et al., 1990). Purticipants were assigned to either a low (1-3) or a high (4-7) LOA. Participants were not stratified for years of education since the elder cohort overall had enjoyed lewer years of education, and matching would have reduced the sample size (see table 1 for subject characteristics).

Table 1: Subject characteristics (means, SD in brackets)

\begin{tabular}{|c|c|c|c|c|c|c|c|c|}
\hline \multirow[b]{2}{*}{ Age } & \multicolumn{2}{|c|}{ young } & \multicolumn{2}{|r|}{ molddlle-aged } & \multirow{2}{*}{ old } & \multirow{2}{*}{$\frac{\text { middle-aged }}{(4)}$} & \multicolumn{2}{|l|}{ old } \\
\hline & 30 & (4) & 43 & (4) & & & 70 & (4) \\
\hline Edac (Yos) & 15 & (3) & 12 & (4) & 9 & (2) & 10 & $(3)$ \\
\hline LOA $(1-9)$ & 5 & (3) & 5 & (2) & 4 & (2) & 5 & (3) \\
\hline $\mathrm{lQ}$ & 114 & $(12)$ & $1 \|$ & (13) & 113 & $(15)$ & 120 & $(11)$ \\
\hline $\operatorname{Mood}(16-45)$ & 20 & (4) & 21 & (5) & 21 & (7) & 20 & (4) \\
\hline Subj health & 4.2 & $(.5)$ & 3.8 & $(.5)$ & 3.6 & $(6)$ & 3.8 & (6) \\
\hline Obje meath & .4 & (.6) & 1.2 & $(1.1)$ & 1.0 & $(1,0)$ & 1.2 & (9) \\
\hline $\mathrm{M} / \mathrm{F}$ & \multicolumn{2}{|c|}{$14 / 14$} & \multicolumn{2}{|c|}{$13 / 12$} & \multicolumn{2}{|c|}{$13 / 12$} & \multicolumn{2}{|c|}{$14 / 16$} \\
\hline $\mathbb{N}$ & \multicolumn{2}{|c|}{28} & \multicolumn{2}{|c|}{25} & \multicolumn{2}{|c|}{28} & \multicolumn{2}{|l|}{30} \\
\hline
\end{tabular}

\section{Procedure}

Subjects filled in a postal questionnaire at home. Then, randomized for time of day, they performed cognitive tests in our laboratory as part of the Maastricht Aging Study (see: Jolles, Houx, van Boxtel, \& Ponds, 1995). First, they did the regular word-learning test (see test description) and after $20 \mathrm{~min}$, in which they performed other cognitive tests, they performed a delayed recall tests. After 30 min of testing and a break of 30 min, they did the word-learning test with distraction. After a further 20 min, in which they did unrelated tests, subjects performed the delayed recall of this test version. In total, subjects were tested for about 3.5 hours.

\section{Mood, health, and education}

Background information was obtained from several sources, including a postal survey and a morbidity checklist. Mood was assessed with the depression subscalle of the Symptom Checklist (SCL-90), which is a widely used multidimensional checklist for psycho-pathological complaints (Arrindell \& Ettema, 1986). Health criteria for subjectively perceiwed health were anssessed with a 5-point scale which ranged from '1" (very bad) to ' 5 ' (very good). Objective health criteria were. also obtained from the postal survey. A selection was made for adwerse health-related factors which are suspected to have detrimental effects on cognitive functions, namely, neurological treatment (e.g. severe brain trauma; any muscular or nervous disease); systemic disease (diabetes, thyroid disease); medication liable to induce cognitive deficits (e.g. benzodiazepines, beta-blockers); alcohol abuse; drug abuse; and neurotoxic substance exposure (for detailed information see: Jolles, Houx, wan Boxtel, \& Ponds 1995). 
The answers to these questions were recoded as ' 0 ' (no health-related factor present) and ' 1 ' (possibly and probably a health-related factor present). Then, an objective health compound score was calculated from the sum of factors present, which ranged from ' $O$ ' (none present) to " 6 ", which indicated that all factors were present.

\section{Cognitive functions}

Cognitive functions were examined with a short cognitive screening battery which included the following tests.

Visual Verbal Learning Test (VVLT) is an improved wersion (Brand \& Jolles, 1985) of a test orginally devised by Rey (1964). A list of 15 monosyllabic meaningfull nouns, matched for high word frequency, was presented on a computer screen connected to an IBM compatible computer at a rate of $1 \mathrm{sec}$ per word (interstimulus interval $2000 \mathrm{msec}$ ). Subjects were asked to verbally recall as many of the wisually presented words as possible as soon as the presentation had stopped. The first trial was followed by four more trials in which the same words were repeated in the same order. The number of correctly recalled words was noted after each trial. Trials 1-3 represent the strongest learning effect while trials 4 and 5 show an asymptotic function. Hence, for analyses of learning we used data from the first three trials only. The naximum number of words immediately recalled over five trials was taken as a measure of immediate recall. After the fifth trial, the subjects completed other cognitiwe tests of nonverbal, memory-unrelated material and after 20 minutes were asked to mention as many of the previously learned, visually presented words (delayed recall) as possible without prompting. The maximum and delayed recall were analyzed together to assess the rate at which items are forgotten from LTM.

Visual Verbal Learning Test with Auditory Distraction. This test is similar to the former except for the following. A different list of 15 monosyllabic nouns was presented on the computer screen at a rate of $\mathbb{s e c}$ per word (interstimulus interval $2000 \mathrm{msec}$ ) (Brand \& Jolles, 1985 ). Simultaneously, 15 similar but different nouns were presented auditorily each $500 \mathrm{msec}$ after the initial target presentation. Subjects were asked to recall verbally as many of the visually presented words as possible as soon as the presentation had stopped without mentioning the (distracting) auditorily presented words. Dependent variables were similar to the ones in the former section.

The Letter-Digit-Substitution Test (LDST). (Jolles, Houx, van Boxtel, \& Ponds, 1995) is a modification of the procedurally identical Digit Symbol Substition Test (Smith, 1968). The number of latters (to each digit) correctly filled in $90 \mathrm{sec}$ was an independent variable to assess the speed of information processing.

Intelligence. The Groninger Intelligence Test (GIT) was used as a test of general intelligence (IQ) (Luteijn \& van der Ploeg, 1983). The GIT is used as much as the WAIS in the Netherlands. The four following subtests are used to arrive at a reliable estinate of the IQ, namely (1) Doing sums (Cijferen) involves the correct completion of as many sums (addition) as possible in $\mathbb{I}$ min (2) Vocabulary (Woordenlijst) is a multiple choice test in which an exact synonym must be chosen for a given word from five alternatives, (3) Mental Rotation (Legkaarten) requires subjects to mentally rotate two dimensional shapes, and (4) Analognes (Woordmatrijzen) is a multiple choice test in which the subject has to indicate which of five words is related in the same way to a given word as two words in an example. 
Table 2 : Tests, parameters, measures, and construm ts of the dependent (VVLT) snd predictor waribles.

\begin{tabular}{|c|c|c|c|}
\hline Test & Parameter & Measure & Comstruct \\
\hline VMLT & $\begin{array}{l}\text { Trial } 1-3 \\
\text { Maximum recall } \\
\text { Delayed recall } \\
\text { Maximum-Delayed recall }\end{array}$ & $\begin{array}{l}\text { Hof of words } \\
\text { of words } \\
\text { w of words } \\
\text { H of words }\end{array}$ & $\begin{array}{l}\text { Learning } \\
\text { Immediate recall } \\
\text { Retrieval ability } \\
\text { Rate of forgetting }\end{array}$ \\
\hline Predict & $\begin{array}{l}\text { variables } \\
\text { Years of education } \\
\text { Adrerse health-related factors } \\
\text { "I feel heathy" } \\
\text { LDST }(90 \mathrm{~s})\end{array}$ & $\begin{array}{l}\text { Years } \\
0-6 \\
1-5 \\
\text { of items }\end{array}$ & $\begin{array}{l}\text { Yeatrs of ctucation oxperienced } \\
\text { objective health } \\
\text { Subyective theath } \\
\text { Speed of information processings }\end{array}$ \\
\hline
\end{tabular}

\section{Statistics}

A $4 \times 2 \times 3$ MANOVA was carried out over the first three learning trials of both word learning tests to assess the effect of age, type of task, and learning, and the interaction between age, type of task, and learning. Also, a $4 \times 2 \times 2$ MANOVA over the two-word-learning test was done with the maximum immediate recall and the delayed recall of the two tests to investigate the effect of age, type of task, rate of forgetting (involving the performance of the maximum immediate recall and the delayed recall), and the interaction effect between age, the type of task, and the rate of forgetting. Then, separate MANOVAs were done for each test.

Hierachical multiple regression analyses were performed to assess the percentage of explatined variance $\left(R^{2}\right)$ of the immediate and delayed recall in both word learning tests separately by age, years of education, objective and subjective health, and speed of information processing (LDST performance), and in combination with age (see table 2 for variables). First, we assessed the association of all separate variables with the dependent variables. Then, we entered separately age with education, with health factors, and with the LDST performance, as a measure of speed of information processing, (see table 4) to investigate whether the effect of age would be partialled out by any of these factors.

\section{Results}

\section{Subject characteristics}

The number of years that subjects had spent at school was significantly different between the age groups, in that the old group overall had had fewer years of education $[E(3,106)=20.1,2<.001]$. Groups did not differ with respect to the general level of intelligence $(\mathrm{GIT})[\mathbb{E}(3,107)=2.6, \mathrm{p}=\mathrm{ns}]$ or the $\operatorname{LOA}[\mathrm{E}(3,102)=28, \mathrm{p}=\mathrm{ns}]$.

There was a significant difference between the reported subjective health over age groups $E(3,107)=6.5, \underline{p}<.001]$. Yet, on average all age groups had scores in the range 3.6 to 4.2 (on the 5-point scale), which indicates that the subjects felt healthy. The number of objective health-related factors was higher in the oldest subjects $[\mathrm{E}(3,1012)=4.23,12<.005]$ allthough the average number of adverse health-related factors experienced was stil low i.e, on average about 1. No differences between groups were found on the mood scale $[\mathrm{E}(3,106)=28, \mathrm{~d}=\mathrm{ns}]$. 


\section{Connitive merasures}

\section{Learning}

A $4 \times 2 \times 3$ MANOVA over the first three immediate recall trials involving the two-word learning tests revealed a significant main effect of age $[F(3,107)=7.81, \mathbb{p}<.0001]$, a main effect of type of test $[E(1,1071)=142.39,0<0001]$, but no interaction beiween age and type of test $[\mathrm{F}(3,107)=.92$, $\mathrm{Q}=\mathrm{ns}$. A significant main effect of learning emerged $[\mathrm{E}(2,106)=526.62, \mathrm{z}<.0001]$, but there was no significant interaction between age and learning $[\mathbb{E}(6,210)=1.77, \mathbb{2}=n s]$. There was a significant interaction between the type of test and learning $[E(2,106)=3.26$, $\mathrm{p}<.05]$ although the three way interaction (age $x$ test $x$ learning) was not significant $[\mathrm{E}(6,210)=1.45, \mathrm{D}=\mathrm{ns}]$.

A separate $4 \times 3$ MANOVA, inwolving the first three trials of the word-learning test without distraction revealed a significant main effect of age $[E(3,107)=7,9, \mathrm{R}<.0001]$, and a main effect of leaming $[\mathrm{E}(2,106)=467.9, \mathrm{p}<.0001]$, but no interaction between age and learning $[\mathrm{E}(6,210)=1.17$, $\mathrm{p}=\mathrm{ns}$ ] (fig la). A 4 × 3 MANOVA over the first three immediate recall trials of the word-learning test with distraction revealed a significant main effect of age $[E(3,107)=4.5, p<005]$ and a main effect of learning $[\mathrm{E}(2,106)=259.8, \mathbb{2}<.000 \mathrm{~d}]$. There was a significant interaction between age and learning $[\mathbb{E}(6,210)=2.26, \mathbb{R}<05](\mathrm{fig} \| \mathrm{b})$.

\section{Rate of forgetting}

A $4 \times 2 \times 2$ MANOVA over the maximum immediate and delayed recall of the two-word learning tests revealed a significant main effect of age $[\mathrm{E}(3,107)=4.47, \mathrm{D}<.005]$, a main effect of type of test $[\mathrm{E}(1,107)=78.57, \mathrm{R}<.0001]$, but no interaction between age and type of test $[\mathrm{E}(3,107)=.71$, $\mathrm{D}=\mathrm{ns}]$. A significant niain effect of the rate of forgetting emerged $[\mathrm{F}(1,107)=371.87, \mathrm{Q}<.0001]$ and also the interaction between age and the rate of forgetting was significant $[E(3,107)=3.36$, $p<05]$. There was a significant interaction between the type of test and the rate of forgetting $[F(1,107)=49.44, p<.0001]$, the three way interaction (age $x$ test $x$ rate of forgetting) was not significant $[\mathrm{E}(3,107)=.14, \mathrm{p}=\mathrm{ns}]$.

A separate $4 \times 2$ MANOVA over maximum and delayed recall of the word-learning test without distraction revealed a main effect of age $[E(3,107)=3.77, \underline{2}<01]$, a main effect of the rate of forgetting $[E(1,107)=160.46,2<.0001]$ but no interaction between age and rate of forgetting $[\mathrm{E}(3,107)=1.99 . \mathbb{R}=n s]$ (fig 2a). By contrast, a $4 \times 2$ separate MANOVA over the maximum and delayed recall of the word-learning test with distraction showed a significant main effect of age $[\mathbb{E}(3,107)=4.15,[2<005]$, a significant main effect of the rate of forgetting $[\mathbb{E}(1,107)=364.15$, $\mathbb{2}<.0001]$ and a significant interaction between age and the rate of forgetting $[\mathrm{F}(3,107)=2.66$, $\mathrm{p}=05$ ) ( fig 2b).

\section{Speed of information processing}

Speed of information processing, as measured with the LDST, was significantly affected by age $[E(3,107)=24,04,2<.001]$ : the young group correctly filled in $58(\mathrm{SD}=8)$ letters in 90 sec, the young middle-aged group $53(\underline{\mathrm{SD}}=6)$, the old middle-aged gronp $48(\underline{\mathrm{SD}}=8)$, and the old group $41(\underline{S D}=9)$.

\footnotetext{
For five subjects, the LOA could not be calcutated

2 Data for six subjects are missing because these subjects did not complete ane or more of the biological life event-related questions
} 


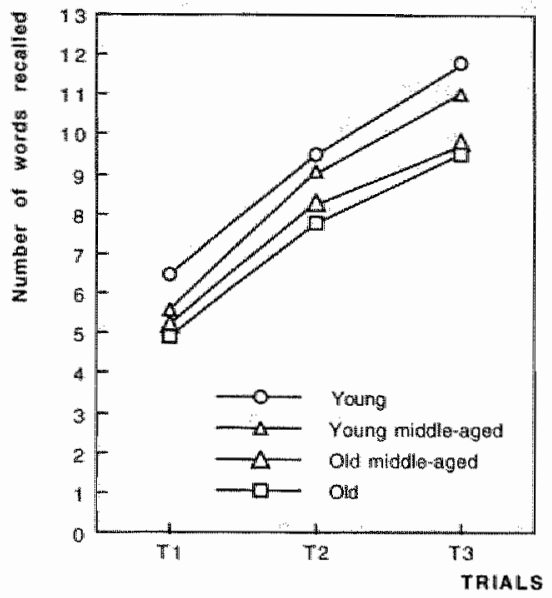

Fig 1a. Leaming in the word learning test without distraction limmediate recall over trialt (T1), trial 2 (T2). trial 3 ( 33 ) I in different age-groups

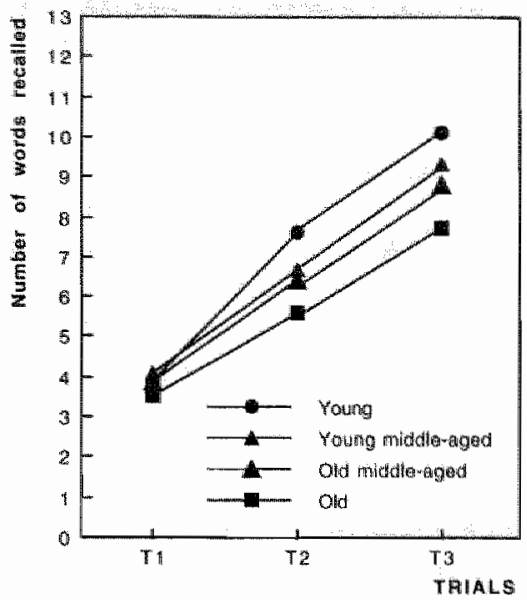

Fig 1b. Leaming in the word learning test with distuaction [immediate ireall ower trial1 (T1). tral 2 (T2), Itlal 3 (T3) ] in different age groups

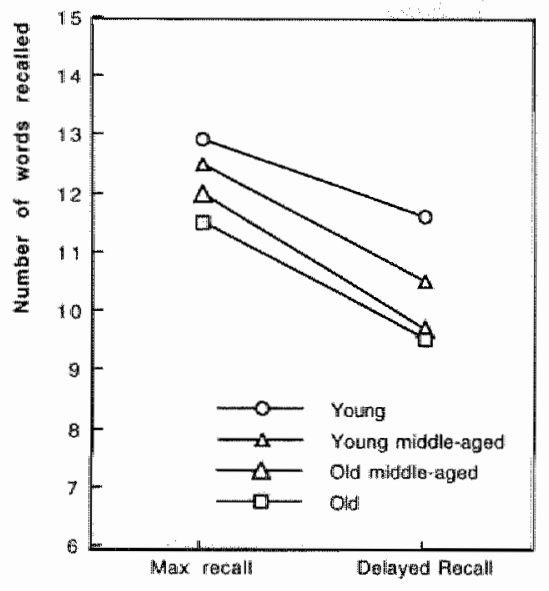

Fig 2a. Forgetting in the mord learning test without distraction (maximum immediate recall over 5 trials versus the delayed ineali) in different age-groups

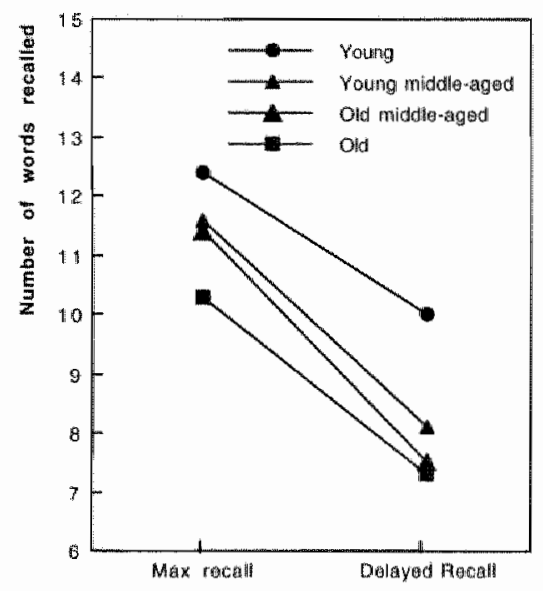

Fig 2t. Forgetting in the word learning tost with distraction (maximum immectlate recall over 5 trieils versus the delayed recall) in different age-groups

\section{Hierachical multiple regression analyses}

To test the mediating effects of years of education, objective and sulbjectiwe health, and speed of information processing on the relation between age and immediate and delayed recall, we performed a series of hierachical multiple regression analyses. Table 3 shows the correlation between the variables.

Hierachical regression analyses revealed that age explained $7 \%$ of the variance in the maximum immediate recall of the word-leaming test without distraction. On the complex word-learning test with distraction, age explained $8 \%$ of the variance. Years of education, objective health, and subjective health when entered with age did not contribute significantly to the variance in any of 
the analysies of the two tests. When the speed of information processing was entered together with age, all agerelated variance was partialled out in the analyses of both tests. The speed factor explained $15 \%$ of the wariance in the maximam immediate recall of the word-learning test without distraction and $17 \%$ of the variance on the maximum immediate recall of the word-learning test word-learning test with distraction (tablle $4 \mathrm{a}$ and $4 \mathrm{~b}$ ).

Table : Petron"s correlation matrices $(p<05 * 0<01)$

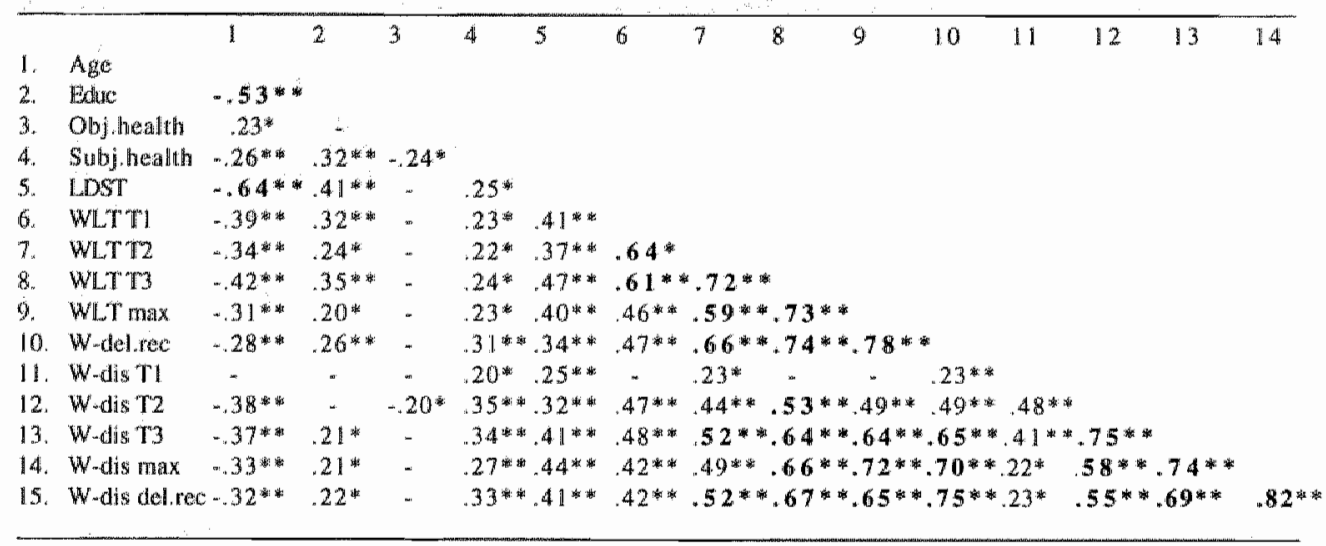

Table 4a: Summary of the simple and Hierachical multiple regression analyses of the maximum

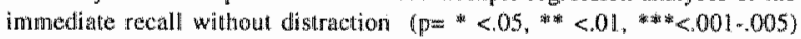

\begin{tabular}{|c|c|c|c|c|c|}
\hline Predictor & B & Cum. $\mathbb{R}^{2}$ & $\mathrm{R}^{2}$ change & $\mathbf{F}$ & \\
\hline Age & $-.26^{3}$ & .07 & & $7.35 *$ & \\
\hline Education & .17 & .03 & & $3.19 \mathrm{~ns}$ & \\
\hline $\mathrm{x} \mathrm{age}$ & $-23^{*}$ & .07 & .00 & $3.75 *$ & (Ecducation $B: 05$, ns) \\
\hline Obj Health & .01 & .00 & & .01 & \\
\hline$x$ age & $\times .27 \%$ & .07 & .00 & $3.78 *$ & (Obj healnh $B-.05, n s$ ) \\
\hline Subjhealth & 20 & .04 & & $4.27 *$ & \\
\hline$x$ age & $-.22^{*}$ & .09 & .02 & 4.84 & (Subj health $(\mathrm{s}: 15,12 \mathrm{~s}$ ) \\
\hline LHSST & 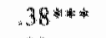 & .15 & & 17.34 & \\
\hline$x$ age & -.03 & .15 & .08 & $8.63^{2}$ & (LDST B:36**) \\
\hline
\end{tabular}

Table 4b : Summary of the Simple and Hirachical multiple regression analyses of the maximum inmediate reall with distraction

\begin{tabular}{|c|c|c|c|c|c|}
\hline Preditetor & B & Cum. $R^{y}$ & R eluange & $\mathrm{F}$ & \\
\hline Age & -.28 * & .08 & & $8.76 \%$ & \\
\hline Education & $19 *$ & .04 & & $3.5 *$ & \\
\hline$x$ age & $4.25 \%$ & .08 & .00 & $4.49 *$ & (Education B:05, n5) \\
\hline Obj. Hedth & $\therefore .01$ & .00 & & .01 & \\
\hline nge & $-.30 *$ & .08 & .00 & $4.66 \%$ & (Obj health $B: 08, \mathrm{~ns}$ ) \\
\hline Subj hoalth & $.22^{*}$ & .05 & & $5.67^{*}$ & \\
\hline$x$ age & -.24 & .11 & .03 & $6.04 *$ & (Subj health $19: 17, p=08$ \\
\hline LDST & $.4 \|^{3 * * *}$ & .17 & & $20.89 *$ & \\
\hline$x$ atge & -.01 & 17 & .09 & $10.40 * *$ & (LDST $\left.B: 39^{* 2 *}\right)$ \\
\hline
\end{tabular}


In analyses of the delayed recall, a different picture emerged. When age was antered allone in the analyses of the word-learning test without distraction, only 4 to of the variance was explatined. Age explained $7 \%$ of the variance in the delayed recall of the memory task with distraction. Aguin, years of education and objective health did not contribute to the variance when entered with age in both analyses. However, subjective health explained $10 \%$ of the variance in the delayed recall of the word list without distraction when entered with age and partialled out the effect of ange: Subjective health accounted for $13 \%$ of the variance in the delayed recall of the word list with distraction when entered with age and also partialled out the effect of age. The speed of information processing explained $9 \%$ of the variance in the delayed recall of the word-learning test without distraction and partialled out the age-related variance. Fifteen percent of the variance in the word-learning test with distraction was explained by speed of information processing and, also here, the effect of age was partialled out.

When the level of subjective health was entered in combination with the speed of information processing, the percentage of explained wariance in the delayed recall of the word-learning test without distraction increased to $13 \%$, and to $20 \%$ in the word-learning test with distraction. Age did not explain the variance in performance of either test (table $5 \mathrm{a}$ and $5 \mathrm{~b}$ ).

Table 5a : Summary of the Simple and Hierachical multiple regression andystes of the delayed recall wilhout distraction

\begin{tabular}{|c|c|c|c|c|c|}
\hline Predictor & $B$ & $\operatorname{Cum} \mathrm{R}^{2}$ & $\mathbb{R}^{2}$ change & $\mathbf{F}$ & \\
\hline Age & $-.21 *$ & .04 & & $4.57 *$ & \\
\hline Education & .244 & .06 & & $6,00 *$ & \\
\hline$x$ age & -11 & .06 & .02 & $3.51 *$ & (Education 0.18, ns) \\
\hline Obj. Heal th & $\cdots, 11$ & .01 & & 1.29 & \\
\hline$x$ rage & -.19 & .05 & .01 & 2.53 & (Obj health $(3:-.07,0 \mathrm{~s})$ \\
\hline Subj health & $.28 *$ & .08 & & $8.32 *$ & \\
\hline$x$ age & -.15 & .10 & .06 & 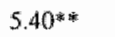 & (Subj health B:.23 *) \\
\hline LDST & $.29 * * *$ & .09 & & $9.50 *$ & \\
\hline age & -.04 & .09 & .05 & $4.76 \%$ & $($ LDST $B: 27 *)$ \\
\hline \multicolumn{6}{|l|}{ LDST } \\
\hline Subj heath & $.22^{4}$ & .13 & .04 & $7.544^{2 *}$ & L DST D:.24 \\
\hline$x$ ange & $.22^{*}$ & .13 & 00 & $4.9 g^{2}$ 事 & $(A g e, 0:-06 n s)$ \\
\hline
\end{tabular}

Table $5 \mathbf{b}$ : Summary of the Simple and Hierachical muliple regression analyses of the defayed recall with distraction

\begin{tabular}{|c|c|c|c|c|c|}
\hline Predictor & $\mathbb{B}$ & $\mathrm{Cum}_{\mathrm{H}} \mathbf{R}^{2}$ & $\mathrm{R}^{2}$ change & $\mathbb{F}$ & \\
\hline $\mathrm{Age}$ & $-27 * *$ & .07 & & $7.80 *$ & \\
\hline Exdection & $.20^{*}$ & .04 & & $4.19^{*}$ & \\
\hline$x$ age & $-.22^{*}$ & .07 & .00 & $4.13 *$ & (Edacation $\mathrm{B}:$ :08, ns) \\
\hline Objl Health & -.11 & .01 & & 1.18 & \\
\hline$x \operatorname{age}$ & $-26^{4 k}$ & .07 & .00 & $4.01 \%$ & (Obj health B: -05 , ns) \\
\hline Subjhealth & $.30 * *$ & .09 & & $10.14 * * *$ & \\
\hline$x$ a a ge & $-.2 I^{*}$ & .13 & .06 & $7.58 * *$ & (Subj health B: 25**) \\
\hline LDST & $.39 * *$ & .15 & & $18.03^{4}$ & \\
\hline$x$ age & -.04 & .15 & .08 & $8.99 \%$ & $\left(\operatorname{LDST}^{4} 8: .36^{4}\right)$ \\
\hline$x$ Subs health & $.22 * *$ & .20 & .05 & 12.35 d w & 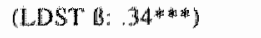 \\
\hline$x$ age & $.22 * \cdots$ & .20 & .00 & $8.15 *$ & (Age $\mathrm{s}: 04 \mathrm{~ns})$ \\
\hline
\end{tabular}




\section{Discussion}

The data of the present study partly confirm our hypotheses. First, we investigated whether the complex word-learning test with distraction was more sensitive to age than the regular word-learning test without distraction. Overall, the analyses revealed no interaction effects of age and type of test on performance measures. However, separate analyses showed interaction effects of age and learning and age and the rate of forgetting in the word-learning test with distraction, while no such interactions were detected in analyses of the word-learning test without distraction.

All groups had a similarly impaired performance in the first trial of the word-learning test with distraction. This may indicate that a floor effect was reached and may explain why, due to the better learning capacity of the young group combined with their llower vulnerability to distraction, an interaction effect was seen between age and learning. Furthermore, all groups had a higher rate of forgetting items from long term memory in the more complex word-learning test than in the word-learning test without distraction. Here, the interaction effect was probably explained by the performance of the two middle-aged groups, which in the delayed recall of the word list with distraction was very close that of the oldest age group and clearly different from that of the young group. This may indicate that the word learning test with distraction is more suitable for detecting the effects of age on learning and rate of forgetting information stored in long term memory than is the regular version, especially when middle-aged subjects are investigated.

Interestingly, we found that age explained a smaller percentage of variance in memory performance than has been reported in other studies ( $\pm 7 \%$ e.g. as compared to $34 \%$, Bryan and Luszcz, 1996, or 55\%, Salthouse and Coon, 1993). The inclusion of the two middle-aged groups may have accounted for the rather small contribution of age to the variance in recall performance. Salthouse and Coon (1993) mentioned that the variance accounted for by age decreases when intermediate, instead of extreme, age groups are used. This may reflect the non lineair effect of aging on cognitive functions, which needs further investigation. Nillson et al (1997) also investigated groups from all age ranges and still found that age accounted for $34 \%$ of the variance in episodic memory. However, they included more very old subjects of 80 years of age in their study, whereas our oldest subject was 77 years of age. Hence, a relatively 'young' old group could also account for the low percentage of variance explained by age in our study.

The number of years that subjects had received education did not overrule the effect of age on recall performance. The general level of intelligence was the same for the age groups studied but the alverage $1 \mathrm{Q}$ score on the GlT was quite high for the oldest group, due to a smaller range (100 10 142). This may indicate that this selected sample was not entirely representative for this age group, which in lum may explain why education did not overnule the age effect. This is important since it has been argued that intellectual capacity rather than chronological age modulates memory performance (Christensen \& Birrell, 1991), which is why subjects are usually matched for years of education or educational level. Again. Nilson at al. (1997) also found that the effect of years of education on memory performance disappeared when age was entered and that education did not alter the age effect in an ANCOVA on memory performance. In addition, we did not find that objective health could explain the age-related variance in performance of the word-learning tests, whereas others have shown that objective adverse health-related factors have a detrimental effect on cognitive functions, especially on the more complex tasks (Houx et al.., 1991. Jolles, Verhey, Riedel, \& Houx, 1995b). Our subjects had experienced few detrimental health events (on average 1), which contrasts with the earlier studies, in which, for instance, on average 2.4 adverse health events were reported (e.g. Houx et al, 1991). The study by Nillson et al 
(1997) rewealed that certain blood biochemistry variables, systolic and diastolic blood pressure. prescribed medication use, and vision were associated with episodic memory performance. However, the observed relationship was fully accounted for by the warible age, and the age-memory relationship was only partially mediated by these objective health parameters. Nillson et al did not find subjective health to be related to memory perfornance. These findings contrast with our finding that subjective health explained some of the variance in the delayed recall perfomance. Similarly, others have found that subjective health partially mediates the relationship between age and cognitive performance related to speed of processing (Garles de Salthouse, 1995). In addition, in the present study the percentage explained variance increased for the more complex memory task. This may indicate that subjectively experienced health, but not objective health-related factors, or years of education, is associated with decreased performance especially in the more complex word learning test.

Craik (1986) stated that memory is a function of both self-initiated processing and environmental support. Earlier fundings (Craik \& Bird, 1982) showed that elderly subjects perform the worst on tasks that contain little envirommental support and that have high processing demands, such as free recall as compared to, for instance, cued recall or recognition. Craik suggested that older adults are deficient in self-initiated and effortful processing because of a decrease in processing resources or "mental energy". Our findings suggest that poorer subjective health may give rise to an inability to employ additionall resources to perform self-initiated activity, such as retrieval of information from long-term memory.

Furthermore, reduced processing resources may result in slower performance of cognitive operations (Salthouse, 1994). As in earlier studies (Bryan \& Luszcz, 1996; Fisk \& War, 1996: Park, et a1, 1996; Salthouse \& Babcock, 1991; Salthouse \& Coon, 1993), we found that the age-related variance in recall performance was largely explained by the speed of information processing. A consistent slowing of performance is seen with increasing age (Poon, 1993). The present and former results suggest that this decrease in the speed of information processing is associated with a decreased ability to store information for a relatively short period of time, as evidenced by the maximum number of words immediately recalled. Also, the speed of information processing was associated with a decrease in dellayed recall or retriewal Memory consolidation functions may be largely dependent on the speed with which information is activated and processed, which may explain the intermediate effect of speed of information processing on memory (Baddeley, 1986; Salthouse \& Babcock, 1991). Other investigators bave used the Digit-Symbol-Substitution-Test (DSST, conceptually similar to the LDST used in the present study) either alone or in combination with other tasks 10 assess the mediating effect of, for instance, "perceptual speed" (Earles et al, 1997; Park et al, 1996). These tests do not only measure perception, but also tap rather complex psychomotor speed functions (reading, writing) as well as working memory functions. Hence, they do not give much information on whether detrimental basic sensorimotor functions account for the age-related variance in memory tasks. For instance, visual acuity and basic visual processing have been found to deteriorate with age (Frahle \& Daum, 1996). Furihermore, some authors (Lindenberger \& Baltes, 1994) have found that sensory dysfunction accounts for much of the age-related variance in cognitive performance. If this is true, the "processing resource" theory is contracticted, since this theory is concerned with cognitive aspects and not with the decline in sensory functions or the slowing of functions dute to muscle deterioration and other peripheral changes with age. In another study, the intermediary effect of sensorimotor and working memory functions on age-related memory performance will be investigated. 
In conclusion, we showed that a complex word-learning test with distraction was more sensitive to the effects of aging at the level of information storage and retrieval compared to the same test without distraction. Subjective health and the slowing of information processing could explain the age-related variance in performance of both memory tasks. Education and objective health did not overrule the effect of age; however, differences in intellectual capacity may have biased these results (selection error). Although the tests were spaced widely apart, the two memory tests had to be dome in one test session; and motivational or other cognitive aspects (e.g. intrusion susceptibility) may have come into play. For this reason we will investigate the effects of education and optimal health, and the intermediary effects of basic sensorimotor speed and working memory on complex memory performance in more detail in another study. 


\title{
Chapter 4
}

\section{The Effects of Age, Basic Sensorimotor Speed and Working Memory in a Word Learning Test with Distraction.}

\author{
Eef Hogervorst, Wim J. Riedel, Peter J. How, Jeroen A. J. Schmitt, \& Jelle Jolles
}

\begin{abstract}
Earlier, we found that the effects of age on memory performance in both simple and more complex memory tasks were partialled out by speed of information processing and subjective health. We investigated this age-related impairment further, using a complex word learning test with distraction. Sixty healthy subjects, aged 26 to 74 years of age, were recruited by means of an advertisement. There was no interaction between age and learning or age and rate of forgetting. Hence, in a healthy population, there is no additional age-related impairment of performance of a complex memory task. The effect of basic sensorimotor speed on recall was mediated by working memory storage capacity. The variance in performance of the delayed recall task was explained by working memory capacity and memory search functions, which partialled out the effect of age.
\end{abstract}

\section{Introduction}

Some forms of learning and memory remain relatively stable with age, while other forms, such as free recall, are clearly impaired (Park et al., 1996; Woodruff-Pak \& Finkbiner, 1995). There are competing theories about the mechanisms underlying these differences (Light, 1991). According to some authors, the age-related memory impairment is due to the higher demand that some memory tasks have on the processing capacity, such as working memory or attentional control (Cerella, Poon, \& Williams, 1980; Craik \& Bird, 1982; Hasher \& Zacks, 1979; Salthouse, 1985; Salthouse, 1994; Salthouse \& Coon, 1993). For instance, there is evidence that there is an age-related vulnerabillity to distraction in a variety of tasks (McDowd \& Birren, 1990), especially if the distraction consists of meaningful material (Carlson, Hasher, Connely, \& Zacks, 1995). It has been hypothesized that elderly people may be more susceptible to distraction because of a diminished ability to screen irrelevant information out of working memory (Gerard, Zacks, Hasher, \& Radwansky, 1991). Indeed, in an earlier study, we found that elderly subjects learned less and had a higher rate of forgetting than younger subjects in a complex visual word learning test, which involved audititory distraction (Hogervorst, Riedel, Houx, Schmitt, \& Jolles, submitted). These age-related differences were not seen in a word learning test without distraction. Furthermore, the age-related variance in delayed recall was partialled out when subjective health and speed of information processing were controlled for in regression analyses.

In the present study, we investigated the effect of health-related factors on the performance of the complex word learning test with distraction. For this purpose, we selected only respondents who considered themselves healthy and who did not have any adverse health-related factors in their medical history. Also, all subjects had had the same number of years of education. To exclude the 
possible effect of interference when performing wo word list tasks we used the complex word learning lest with distraction only. In our other study, we found that the speed of information procegsing partialled out the age-related variance in memory performance. Yet, it has been suggested that the speed of information processing affects memory functions indirectly through its effect on working memory (Salthouse \& Babcock, 1991). For instance, Fisk and War (1996) found that controlling for speed of processing removed all age-related wariance in working memory. Bryan and Luscz (1996) found that working memory explained only $3 \%$ of the wariance in recall after speed of processing was controlled for in regression analyses. Again, Park et al. (1996) suggested that the contribution of working memory varies as a function of the type of memory task: when memory becontes more effortful, the cantribution of working memory increases. Fastenau, Denburg \& Abeles (1996) suggested that the contribution of working memory to variance is task dependent, since they found that the age effect on long-term verbal memory performance was reduced by $60 \%$ when working memory was controlled for. By contrast, in visual reproduction task, both speed of processing and working memory were found to overrule the age-related variance. Kirasic, Allen, Shannon, Dobson, \& Binder (1996) also reported that working memory was the major mediator in declarative learning, whereas the contribution of processing speed was small.

Apart from the type of memory task, another complicating factor is that the decline in cognitive performance may be mediated for a large part by a decline in sensorimotor functions (Lindenberger \& Baltes, 1994). Hence, in the present study we included a memory scanning test based on the Stemberg paradigm (Sternberg, 1969). In this test, the 1-intercept (response time for one target) is considered to be a valid measure of basic sensorimotor speed, while the slope (response time by memory set size function) is considered to be a measure of the speed of searching working memory. Working memory is a limited capacity system and involves both the processing and storage of information (Baddeley \& Hitch, 1974). The age-related decline in memory functions is often attributed to a decline in processing resources (Salthouse \& Babcock, 1991), but the storage capacity of working memory also decreases with age (Hasher \& Zacks, 1988). For this reason we used the first trial of the word learning test as a factor to assess the effect of working memory storage capacity. These factors were entered in regression analyses with age to investigate the coniribution of sensorimotor processing speed and working memory functions on immediate and delayed recall. We hypothesized that working memory functions and not sensorimotor speed would explain the age-related variance in performance of this complex memory task.

\section{Method}

\section{Participants}

Sixty healthy subjects aged 26 to 74 were recruited by means of an advertisement in a local newspaper. There was a preselection by telephone in order to exclude subjects with any health-related problems' (Houx, Vreeling, \& Jolles, 1991). The health-related factors sereened for are listed in table 1.

1 Of the 69 people who responded to the awertisement, 60 were included in the study:

2 Dath for four subjects (three in the young group, one in the middhe aged group) were missing because of appa failluio.

3 Vears of education were not different between groups [F(2,53)=1.5, $\mathrm{p}=\mathrm{ns}$ ]. 
Table 1: Exclusion criteria.

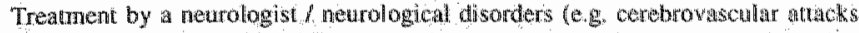

(CVA or TIA) epilepsy: Parkinson's disetse, multiple sclerosis ar any oher muscle or

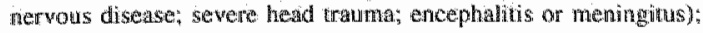

Treament by an internist; diabetes; thyroid disease and renal dialysis

Severe psychatric disturbanees (a depression and alecroshock treatincnt);

The use of medication (or drugs) liable to induce cognitive deficils:

The excessive use of alcohol ( 40 consumptionis/werek);

Uncorrectable motor; wisual; or anditory deficits.

Subjects were matched for sex and level of occupational achievement (LOA) (see table 2 for subject characteristics2). For this purpose, occupation was given a 7-point score based upon at detailed description of the function (Brand et al, 1990). Each group contained subjects with 10 low and 10 high LOA's and ranged from ' 1 ' (e.g., housekeeper, no education) to ' 7 ' (e.g." completed university degree). Subjects were paid the equivalent of 10 dollars for their participation.

Table 2: Means (SD) and range of subject characteristcs for young, middle-aged and olld participants

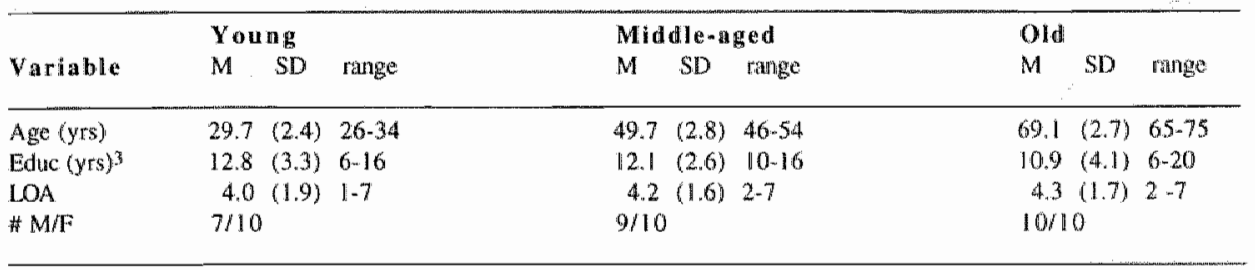

\section{Procedure}

Groups were balanced for time of testing to eliminate the influence of circadian factors. All testing was done in the morning. The entire procedure took 1.5 hours. Subjects were asked not 10 drink any alcohol-containing beverages 24 hour before testing and to arriwe at the laboratory well rested. Subjects recieved a 15 -min training in the testing procedure. After the training, the subjects started the test procedure, which lasited approximately 30 minutes.

\section{Performance tests}

Visual Verbat (Word) Learning Test with Auditory Distraction (VVLT). A list of 15 monosyllabic meaningful nouns matched for word frequency was presented on a computer screen connected to an IBM compatible computer at a rate of $1 \mathrm{sec}$ per word (interstimulus interval 2000 msec) (Brand \& Jolles, 1985). Simultaneously, 15 similar but different nouns were presented auditorily, each 500 msec after the initial target presentation. Subjects were asked to recall verbally as many of the visually presented words as possible as soon as the presentation had stopped; they were not to recall the (distracting) auditorily presented words. The first trial was followed by four more trials in which the same words were repeated in the same order. The number of correctly recalled words was noted after each trial. The number of words immediately recalled ower the first three trials was taken as a measure of learning. After the fifth trial, the subjects completed other tests and after 20 min were asked to mention as many of the previously learned visually presented words (delayed recall) as possible. The maximum and delayed recall were analyzed together to assess the rate at which items were forgotten from long-term memory (LTM). 
Memory Scanming. Subjects were briefly shown a set of letters and told to memorize them. This is called the "memory set" (Brand \& Jolles, 1987; Sternberg, 1969). Subjects were then shown a series of 48 letters displayed for $1000 \mathrm{msec}$ on a computer screen, connected to an IBM compatible computer, followed by another $2000 \mathrm{msec}$ before the next letter appeared. Half of the presented letters were part of the memory set and had to be recognized as targets. The subjects' task was to decide and respond as rapidly as possible by pressing either 'YES' or "NO' to indicate whether or not each successive letter was one of those contained in the memory set. The median of the response time (RT) of correct responses (on targets and non targets) was taken as a dependent parameter. This task was performed with memory sets consisting of 1,2 and 3 letters, respectively. Individual $\mathrm{RT} \times$ set size functions were calculated. This function is defined by the equation $\mathrm{RT}=\mathrm{A} * \mathrm{~S} s+\mathrm{B}$, in which: $\mathrm{A}=$ slope of the function (i.e. the amount of extra time needed per item in the memory set), $\mathrm{S} s=$ size of the memory set, and $\mathrm{B}=$ intercept with the $\mathrm{Y}$-axis. The slope is a measure of speed of scanning working memory whereas the intercept measures basic sensorimotor processing speed (see table 3 for dependent wariables).

\section{Design and statistics}

To assess the effects of age on the dependent variables, we used a parallel groups design. The subjects were allocated to one of three age-groups: young (26-34 years of age), middle-aged (46-54 years of age), and old (66-74 years of age). For trials 1 to 3 of the immediate recall of the word learning test, a MANOVA was carried out to investigate the interaction between learning and age. Also, the rate of forgetting was assessed with a MANOVA over the maximum number of words recalled immediately after five trials and the delayed recall. To assess the extent to which basic sensorimotor processing speed, speed of searching working memory , and working memory capacity mediated the relationship between age and memory performance, we performed hierachical multiple regression analyses, as done in other studies (e.g. Bryan \& Luszcz, 1996), on the maximum of words recalled immediately and the delayed recall.

Table 3 : Tests, parameters, measures and constructs of the elependent and pedicior variables.

\begin{tabular}{|c|c|c|c|}
\hline Test & Parameter & Measure & Construct \\
\hline VVLT & $\begin{array}{l}\text { Trial } \\
\text { Trial } 1-3 \\
\text { Maximum recall } \\
\text { Delayed recall }\end{array}$ & $\begin{array}{l}\text { W of words } \\
\text { of words } \\
\text { Hof words } \\
\text { of words }\end{array}$ & $\begin{array}{l}\text { Working memory storage copacity } \\
\text { Learning } \\
\text { Immediate recall } \\
\text { Retrieval }\end{array}$ \\
\hline Memor & $\begin{array}{l}\text { Scanning "Test } \\
\text { Intercept } \\
\text { Slope }\end{array}$ & $\begin{array}{l}\text { RT in msec } \\
\text { RT' ir msec }\end{array}$ & $\begin{array}{l}\text { Basic sensorimotor processing speed } \\
\text { Speed of seatrching working menory }\end{array}$ \\
\hline
\end{tabular}

\section{Results}

\section{Learning}

A $3 \times 3$ MANOVA over the first three immediate recall trials of the VVLT revealed a significant main effect of age $\left[F\left(2,3^{2}\right)=3.47, \underline{p}<.05\right]$ and a main effect of learning $[F(2,52)=333.72$, $\mathrm{p}<.00011$. However, there was no interaction between age and learning $[\mathrm{E}(4,102)=.09, \mathrm{p}=\mathrm{ns}]$, which means that learning curves did not differ between groups, although the middle-aged and old group had a lower overall performance than the young group (fig la) 


\section{Rate of forgetting}

A 3 × 2 MANOVA over the maximum immediate and the delayed recall of the VVLT revealed significant main effect of age $[\mathrm{E}(2,53)=3.86,2<05]$ and a main effect of the rate of forgetting $[\mathrm{F}(2,53)=90.22, \mathrm{p}<.0001]$. However, there was mo interaction between age and the rate of forgetting $[\mathrm{F}(2,53)=.09, \mathrm{p}=\mathrm{ns}]$, although the middle-aged and ofd group had a lower overall performance than the young group (fig $\mathrm{lb}$ ).

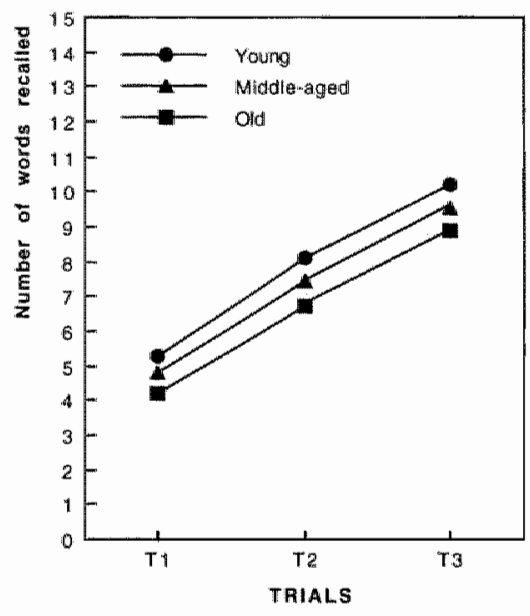

Fig 1a. Leaming in the wiord learning lest with distraction [immediate recall ower trial1 (T1), trial 2 (T2). trial 3 (T3)] in different age-groups

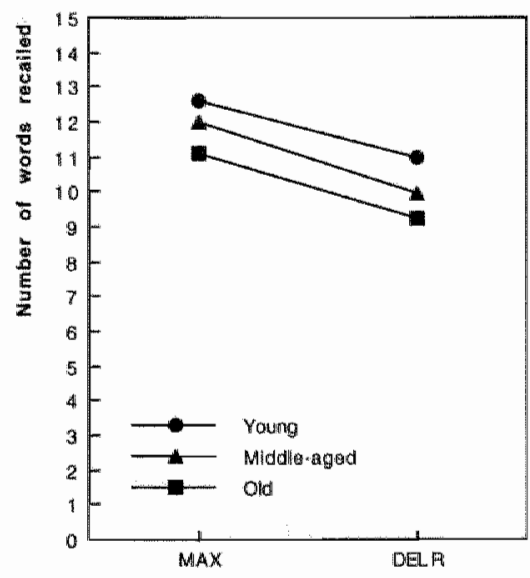

Fig to. Fatte of forgelting in this word learning test with distraction [maximum immodiate recall (max) versus delayed recall] in different ago groups

\section{Predictor variables}

The first trial of the VVLT, as a measure of working memory storage capacity did not show a significant age effect $[\mathbb{E}(2,53)=2.41, \mathrm{p}=\mathrm{ns}]$ : the young group recalled on average 5.3 words $(\mathrm{SD}=1.7)$, the middle-aged $4.8(\mathrm{SD}=1.4)$, and the old $4.2(\mathrm{SD}=1.5)$. The basic sensoninotor processing speed, as measured by the intercept of the memory scanning test, revealed a significant effect of age (table 4). Independent t-tests revealled significant differences between young and old subjects $[\mathrm{t}(35)=-4.14, \mathrm{p}<.0001]$, and between middle-aged and old subjects $[\mathrm{t}(37)=-2.67, \mathrm{p}<.01]$ while no difference was seen between young and middle-aged subjects $[1(34)=-2.15,2=n s]$. No effect of age was detected on the speed of searching working memory, as measured by the sllope of the memory scanning test $[\mathrm{E}(2,53)=51, \mathrm{p}=\mathrm{ns}]$.

Table 4: Outcome (means and SD in brackess of the silope and intercept over the different age groups.

\begin{tabular}{llllll}
\hline Parameter & Young & Middle & Old & F & P \\
\hline Slope & & & & & \\
Lntercept & $48(18)$ & $47(16)$ & $52(17)$ & 51 & $\pi 5$ \\
Linearity & $426(43)$ & $456(43)$ & $506(70)$ & 10.55 & $<0001$ \\
\hline
\end{tabular}


There was a strong correlation between the first trial of the immediate recall and the delayed recall (table 5). Sensorimotor speed had the strongest correlation with age, whereas the speed of searehing working memory did not correlate with age, but modestly correlated with the delayed recall.

Table 5: Pearsons cothetations between age group, speed of semching working nemony (slope), sensorimotor processinger sped (intereepl), worling menory storage capacity (WLTi), maximum immediate recall (WMAX), and delayed recall.

\begin{tabular}{|c|c|c|c|c|c|}
\hline Measure & Age & Slope & latercept & WLTI & WMAX \\
\hline Slope & .09 & & & & \\
\hline Jutercept & .55 & -26 & & & \\
\hline WLT'I & 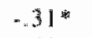 & -.14 & ${ }_{-.} 34^{1+k}$ & & \\
\hline WMAX & $-.39 *$ & -26 & -.15 & $.49^{\circ}$ & \\
\hline Delayed Recall &. $.29 *$ & $-.36 *$ & -.05 & $47^{*}$ & $.78 * *$ \\
\hline
\end{tabular}

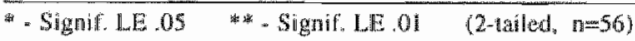

\section{Hierachical multiple regression analyses}

To test the mediating effects of basic sensorimotor processing speed, speed of searching working memory, and working memory storage on the relation between age and immediate and delayed recall, we performed a series of hierachicall multiple regression analyses. The results of these analyses are presented in tables 6 a-c.

Table 6a : Herachical multiple regression analyses predicting the immediate recall in the first trial from age group, besic sensorimotor processing speed (intercept). and speed of searching working memory (stope). Increment in $\mathrm{R}^{2}$ for ange after controlling for other variables is given.

\begin{tabular}{|c|c|c|c|c|c|}
\hline Equation & Variable & $\mathbf{R}^{2}$ & Inc. $R^{2}$ & $\bar{F}$-value & \\
\hline 1 & age & .08 & .08 & $4.90 *$ & \\
\hline \multirow[t]{2}{*}{2.} & intercept: & .12 & 12 & $7.27 *$ & \\
\hline & interedplogge & .13 & $0 !$ & $4.11 \%$ & (ntatere + age ns) \\
\hline \multirow[t]{2}{*}{3.} & slope & .02 & .02 & $1.07 \mathrm{~ns}$ & (stope ns) \\
\hline & slope/age & .10 & .02 & $2.78 \mathrm{~ns}$ & $($ slope ns) \\
\hline 4. & intercept/slope & 18 & .06 & $5.61 * *$ & (slope ns .06) \\
\hline 5 & shope/thiereeptage & .18 & .00 & $3.75 *$ & (slope +age ns) \\
\hline
\end{tabular}

$<05,<01 ; * 001.001,<$

Age accounted for only $8 \%$ of the variance in the first trial of the VVLT when entered first (equation 1). Basic sensorimotor processing speed explained $12 \%$ of the variance in performance of the first trial of the word list (equation 2) and reduced the age-related variance by $88 \%$. However, when both age and sensorimotor processing speed were entered, neither variable had a significant effect, suggesting that basic sensorimotor processing speed alone mainly accounted for the variance in performance of this task. Speed of searching working memory did not make a significant contribution to explained variance, either alone or when combined with age (equation 3) and/ or sensorimotor speed (equations 4 and 5). 


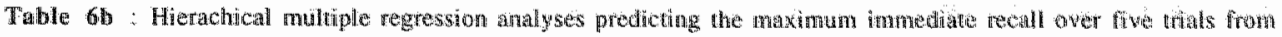
age group, number of words recalled after trial 1 , basic sensorimotor processing sped (intabept, and speed of searching working memory (stope). Increment in $\mathrm{k}^{2}$ for age after controlling for other warbbles is given.

\begin{tabular}{|c|c|c|c|c|c|}
\hline Equation & Variable & $\mathbb{R}^{2}$ & $\operatorname{Int} \mathbb{R}^{2}$ & 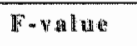 & \\
\hline 1. & age & .15 & .15 & 9.82 & \\
\hline \multirow[t]{2}{*}{2.} & intercept & .02 & .02 & $1.16 \mathrm{~ns}$ & \\
\hline & interceptage & .16 & .01 & $507 *$ & (Antercept ns) \\
\hline \multirow[t]{2}{*}{3.} & slope & .07 & .07 & 4.05 & \\
\hline & slope/age & .21 & .06 & $6.94 *$ & \\
\hline \multirow[t]{2}{*}{4.} & witt 1 & .24 & .24 & $16.89 *$ & \\
\hline & wlthage & .30 & .15 & $11.52 *$ & \\
\hline 5. & age/wituslope & .34 & .04 & $8.85 * *$ & (age ns) \\
\hline
\end{tabular}

Age accounted for $15 \%$ of the variance in the maximum number of words immediately recalled after five trials, (table 6b). The basic sensorimotor processing speed (1-intercept) did not contribute to the variance (equation 2). The speed of searching working memory explained $7 \%$ of the variance and increased the variance explained by age by $6 \%$ to $21 \%$ (equation 3). The number of words recalled in the first trial was the best predictor for the maximum number of words recalled after fiwe trials. This variable accounted for $23 \%$ of the variance in maximum immediate recall performance (equation 4) and increased the variance explained by age to $30 \%$. The effect of searching working memory was partialled out when entered into the regression analysis together with the number of words immediately recalled in the first trial and age (equation 5). Hence, immediate recall was best predicted by working memory storage capacity and age.

Table 6c: Hierachical multiple regression analyses predicting the delayed recall over five trials from age group, number of words recalled after trial 1, basic sensorimotor processing speed (intercept), and speed of searching working nemory (slope). Increment in $\mathrm{R}^{2}$ for age after controlling for other variables is given.

\begin{tabular}{|c|c|c|c|c|}
\hline Equation & Variable & $R^{2}$ & Ine. & Fovalut \\
\hline 1. & $\mathrm{age}$ & .08 & .0 & $4.88 *$ \\
\hline \multirow[t]{2}{*}{2.} & intercept & .003 & .003 & $.15 \mathrm{~ns}$ \\
\hline & interceplage & .10 & .02 & $2.91 \mathrm{~ns}$ \\
\hline \multirow[t]{2}{*}{3} & slope & .13 & .13 & $8.20 *$ \\
\hline & slopelage & .20 & .07 & $6.57 *$ \\
\hline \multirow[t]{2}{*}{4.} & witl & .22 & .22 & 14.98 * * : th \\
\hline & wltl/age & .24 & .02 & $8.39 * *$ \\
\hline 5. & age/wlit/sloge & .33 & .09 & $8.44 * *$ \\
\hline
\end{tabular}

Age accounted for only $8 \%$ of the variance in delayed recall performance when entered first in the regression analysis (equation 1). Sensorimotor speed did not significantly contribute to the variance in delayed recall or reduce the age-related variance (equation 2). The speed of searching working memory accounted for $13 \%$ of the variance and did not partial out the effect of age, but reduced it by $2 \%$ (equation 3 ). The first trial of the immediate recall predicted $22 \%$ of the variance (equation 4 ) and reduced the effect of age from 8 to $2 \%$. When speed of searching working memory was combined with working memory storage, $33 \%$ of the variance in the delayed recall performance was explained and the effect of age was partialled out. Hence, delayed 
recall performance can be explained in terms of memory storage and memory search functions and not by basic sensonimotor processing speed.

\section{Discussion}

Our results show that there was no interaction between age and learning or age and the rate at which items were forgotten in the word learning test with distraction. This suggests that distraction did not additionally interfere with memory retention and retrieval in the elderly subjects. Furthermore, our hypothesis that working memory, but not age, explained the age-related variance in recall performance wass confirmed. By contrast, in our former study (Hogervorst et all, submitted), age and learning, and age and rate of forgetting were shown to interact in this test. How can differences between the findings of the present and the former study be explained?

It seems plausible that the method of subject selection accounts for these differences. The subjects who participated in this study had to meet rigorous criteria for good health. Previous studies have shown that adverse health factors (e.g. alcohol abuse etc.) contribute to a large extent to a decrease in cognitive functions, especially on complex tasks (Houx et al., 1991; Jolles, Verhey, Riedel, \& Houx, 1995). However, when similar objective health criteria were controlled for in regression analyses in our other study, they did not contribute to the variance in recall performance. 'Therefore, differences in 'objective health' do not explain the differences between the two studies. The advertisement for recruiting subjects for the present study specifically asked for healthy subjects only. Although the subjects in our other study also considered on average their health to be good to very good, subjective health was seen to explain some of the variance in delayed recall. Hence, subject presellection (only subjects who considered themselves healthy) may be responsible for some of the differences found between the two studies. Moreover, self-selection may also induce motivational differences, in that subjects are more motivated to show a better performance. Obviously, the latter factor is difficult to control for.

We found that working memory storage capacity and age explained the variance in maximum immediate recall. However, in the delayed recall performance, the effect of age was partialled out by working memory storage, as measured with the first trial of the word learning test, and speed of searching working memory, as measured with the slope. Basic sensorimotor processing speed (1-intercept) did not contribute to the variance in the maximum immediate and delayed recall. However, basic sensorimotor speed did explain some of the variance in performance in the first trial. These data thus suggest that basic sensorimotor speed affects memory functions indirectly, through working memory functions. Earlier studies into the mediator effect of speed of processing on memory (Bryan \& Luscz, 1996; Park et al, 1996; Salthouse, 1983; Salthouse \& Coon, 1993) measured single triall immediate recall and not delayed recall. We found that: immediate recall of the first trial, but not delayed recall, was affected to some extent by the speed of information processing.

In the other studies, the percentage of variance in memory performance explained by speed of processing was much larger, ranging from $36 \%$ (Bryan \& Luscz, 1996) to $55 \%$ (Salthouse, 1993). Kirasic, Allen, Shannon, Dobson, \& Binder (1996), who also found that the contribution of processing speed to declarative learning was small, suggested that the smaller magnitude of the speed factor may have been the result of the type of task used to assess information processing speed. Indeed, in our other study, speed of processing, as measured with the Letter-Digit-Substitution-Test (LDST), contributed to the variance in both maximum immediate and delayed recall. However, the LDST, or the conceptwally similar Digit-Symbol-SubstitutionTest (DSST), may measure more complex information processing speed, since performance on 
the DSST or LDST involves both sensorimotor (reading, writing) and working memory (temporary storage) functions. By contrast, the 1 -intercept is a measure of sensorimotor processing speed does not involve memory functions (Sternberg, 1969). This is of importance, because Lindenberger and Baltes (1994) suggested that the decline in cognitive performance may be mediated by the decline in sensorimotor functions. However, our results suggest that this is not the case for healthy, well screened subjects.

Lastly, other factors may explain the variance in performance of the more complex word learning test. Since the modified task we used demands sellective attention to ignore the distracting information, executive functions could explain the variance in performance in this test. Although Baddeley (1986) suggested that central excutive functions may be impaired in elderly people, Bryan and Lusze (1996) did not find a specific breakdown in central excutive functions with age. We also did not find our older subjects to have additional impaired learning of the difficult word learning test, which required the inhibition of distracting stimuli. The learning curves were similar to those of young and middle-aged subjects, but had a lower intercept (trial 1), which could be partly explained by the slower sensorimotor processing speed of our old subjects. Park et at (1996) also implied that susceptibility to interference is not a strong indicator for age-related differences. Similarly, Perry \& Wingfield (1994) did mot find an additional age-related vulnerability to distraction. When taken together with the earlier findings of an age-related vulnerabilitty to distraction on a variety of tasks (McDowd \& Birren, 1990), the present and former results suggest that subject selection may be responsible for the different findings of various studies. In this and our other study (Hogervorst et al, submitted) we found that subjective health may play an important role in memory performance.

In conclusion, in a healthy, self-selected population, the age-related difference in long-term memory functions, measured with a complex word learning test with distraction, could be explained by a decreased capacity to retain information and a slower speed of searching working memory, and not by a slowing of basic sensorimotor functions. Furthermore, our findings indicate that subjective health, or an experienced physical and mental fitness or vitality, can also explain to some extent the age-related decrease in memory performance, especially on complex tasks. 


\title{
Chapter 5
}

\section{The Effects of Mild Hypoxia and Overbreathing on Cognitive Dysfunction}

\author{
E. Hogervorst, W. J. Riedel, P. Hameleers, S. Sej, M. P. J. v. Boxtel, P. J. Visser, \& J. Jolles
}

\begin{abstract}
This study evaluated experimentally induced mild hypoxia and hypocapnic hyperventilation as models for cognitive deficiency. Twenty male college students were tested according to an order balanced, single blind, controlled cross-over design. Subjects received hypoxic air $\left(13.6 \% \mathrm{O}_{2}\right)$ or normal air (21\%, normoxia) through a mask as a control. One week later, they underwent hypocapnic (end tidal $\mathrm{CO}_{2}<2.5 \mathrm{kPa}$ ) or normocapnic hyperventilation ( $\pm 5 \mathrm{kPa}$ ). No treatment effects were found on memory tasks. Mild hypoxia showed a decrease in visual sensitivity and an increase in the reaction time (RT) of the incompatible choice RT task as compared to normoxia. The effect of hypocapnic hyperwentilation on psychomotor speed dysfunctions could be attributed to the effects of overbreathing (normocapnic hyperventilation). In addition, the wearing a mask (normoxia) also impaired performance of the choice RT task and of sensorimotor processing speed. It was concluded that studies that have not used control treatments in either of the two models should be regarded with caution.
\end{abstract}

\section{Introduction}

There is an interest in effective experimental models for cognitive deficiency in order to evaluate the effect of putative cognition enhancing drugs. Effective drug treatments are needed for Alzheimer"s disease and related conditions in which cognitive function is compromised. Cognitive dysfunction in non-demented subjects (e.g. Age-Associated Memory Dysfunction or Age Consistent Memory Impairment) is - quantitatively - an even more important target area for cognition enhancing drugs (Jolles, Verhey, Riedel, \& Houx, 1995). Unfortunately, there are few thoroughly evaluated models of cognitive dysfunction to test the potential effect of cognition enhancers in homogeneous groups of young volunteers.

The hypoxia model is considered to be an adequate method to test cognition enhancing cerebroactive (e.g antihypoxidotic-nootropic) drugs in healthy volunteers (Maier-Lenz, Ringwelski, Klein, \& Richter, 1980). The hypoxia model aims at mimicking the cognitive dysfunction caused by the decreased oxygen availability in the brain as a result of aging. Hypoxia is induced by letting subjects breath air with a low oxygen content through a mask or by testing subjects at: (simulated) high altitudes. This model has been used to test the therapeutic efficacy of noötropic drugs, such as aniracetam and cyclandelate, on impaired cognitive performance (e.g. Satetu \& Grunberger, 1984; Schlaffer, Hörmann, \& Arnold, 1981). However, since compensatory effects occur during the breathing of hypoxic air (Ellingsen, Hauge, Nicolaysen, Thoresen, \& Walløe, 1987), some authors have proposed that standardized voluntary hypocapnic hyperventilation (HV) would be a better and safer model than hypoxia to test the effect of putative cognition 
whancing drugs (Kraaier, wan Huffelen, \& Wieneke, 1988;1989; Rother, Kessler, Funke, Rother, \& Windisch, 1994). Others have questioned the cognitive effects of hypocapnic hyperventilation, since control procedures were often not used in the evalmation of the cognitive effects of hyperventilation (e.g. Garssen, 1986). The cognitive effects of hyperwentilation were attributed to overbreathing or to cramps which can occur during hypocapnic hyperventilation, and which may hinder the adequate performance of (psychomotor) taskss. Further, although cerebral blood flow is reduced by $40-60 \%$ during hypocapnic hyperventilation, it is unclear whether hypoxia occurs (Kennealy, McLennan, Loudon, \& McLaurin, 1980; Albrecht, Miletich, \& Ruttle, 1986).

In the present study the effects of mild hypoxia and hypocapnic hyperventilation were compared on a number of cognitive functions. In this respect, mild hypoxia is most interesting, since its cognitive effects are disputed (Bahrke \& Shukitt, 1993; Ernsting, 1984) and in more severe hypoxia ( $>5000 \mathrm{~m}$ ) potentially dangerous adverse cardiac side-effects are noted (Kida \& Imai, 1993; Kraaier, 1988; Saletu et al, 1984). Mild to moderate hypoxia (11\%-16\% oxygen, comparable with air at a height of $2400-4500 \mathrm{~m}$ ) clearly affects visual sensitivity and choice reaction time tasks (McFarland. 1963; Fowler, Taylor, \& Porlier, 1987), but has shown variable effects on memory functions (Crow \& Kelman, 1971;1973; Denison, Ledwitli, \& Poulton, 1966; Fowler, Paul, Porlier, Elcombe, \& Taylor, 1985; Fowler, Prlic, \& Brabant, 1994). Hypocapnic hyperventilation has been found to affect working memory functions (Kraaier et al, 1988; Rother et al, 1994) but the avialable evidence seems rather weak. Kraaier et al (1988) did not statistically evaluate their findings and control procedures (for the effects of owerbreathing) were not used in both studies. In an earlier controlled study, hypocapnic hyperventilation failed to have an effect on memory performance as measured with the Wechsler memory test (Murrin \& Nagarajan, 1974). Yet, other studies that controlled for the effect of overbreathing did find a decline in motor coordination (Gibson, 1978), choice reaction time (Stoddart, 1967), and character detection (Nikolov, Patchev ${ }_{*}$ Kisslekova, \& Harleva, 1991).

In the present study, control procedures were used for both treatments, i.e. normocapnic hyperventilation for hypocapnic hyperventilation, to avoid possible confounding effects of strong, forced ventilation. Likewise, normoxia was used as a control procedure to check for the effect of breathing through a mask. Working memory and long term memory, visual sensitivity and choice reaction time were used as dependent variables. Since large interindividual differences in oxygen saturation exist (Kraaier, 1988), a cross-over design was used which enabled the ewaluation of the effects of treatment with subjects serving as their own control. To further minimize error variance, only males were included in the present study, since females have a higher cerebral blood flow during hyperventilation (Kraaier, 1988).

Hypotheses were that mild hypoxia would have smaller effects on psychomotor speed and memory functions than hypocapnic hyperventilation. Furthermore, it was expected that mild hypoxiat and hypocapnic hyperventilation would have detrimental effects as compared to control procedures on psychomotor speed (visual sensitivity and choice reaction time) but not on memory functions

\section{Method}

\section{Subjects}

Twenty non-smoking male volunteers were recruited by means of an advertisement. Subjects were aged between 23 and 30 years (mean age 24 years). and were free of cardiac, hepatic, renal, pulmonary, neurological, gastrointestinal, heamatological, and psychiatric disease as determined by extensive medical screening, including history taking, physical examination, and routine ECG. 
The study was approved by the standing Medical Ethics Conmittee of the University. Subjects gave their written informed consent after reading "Information for volunteers" and were paid $\$ 75$. Dersign

The study was carried out according to a two factor cross-over design (1. hypoxia versus hypocapnic hyperventilation and 2. active versus control versus baseline performanoe). Subjects underwent all treatments, but were randomly assigned to start with either one of the two treatments. Thus, they either started with hypoxia or hypocapnic hyperventilation (each with their own control procedure: normoxia or nomocapnic hyperventilation), and I week later they received the other treatment. The order of normocapnic hyperventilation, hypocapnic hyperventilation, normoxia, and hypoxia was partially counterbalanced, according to a $8 \times 3$ Latin square. Baseline measurements without the mask were always made between treatment and. control procedures. Subjects did not know which treatment they received (single blind) and not did they correctly quess which treatment they had received (of the 80 possible answers, 27 were correct statements, 33 were incorrect, and 20 were inconclusive).

\section{Procedure}

Prior to the experiment, participants trained breathing hypoxic air through the mask (as at challenge procedure), and practiced performing the cognitive tasks. Prior to testing, subjects werte asked to keep to their normal sleeping pattern and to refrain from taking any medication. including "social" dnags (e.g. caffeine, marijuana) and alcohol (the latter within 24 hours prior to the experiment). The procedure on testing days was as follows (see table 1). All testing was done in the morning to avoid circadian variance. After a short practice period, subjects started to learn at list of 15 words in five consecutive presentation trials (Visual Verbal Learning Task, see below for description). Then, the mask was put on. In the subsequent 10 minutes no tests were performed to allow for physiological adaptation, to attain stable levels of $\mathrm{SaO}_{2}$ and end tidal $\mathrm{pCO}_{2}$, and to avoid the influence of early - primarily stress-related - responses (Hout van den de Jong, Zandbergen, \&. Merckelbach, 1990). In the next $30 \mathrm{~min}$, the subjects performed the remaining cognitive tasks (Sternberg memory scanning task, the motor choice reaction time task and the wisual detection task, see below for description). After completion of these tasks, the subjects returned to normal breathing in 5 min. The mask was taken off. Then, the subjects were tested for delayed recall of the words learned prior to the hypoxia or hyperwentilation treatments (Visual Verbal Learming Tasky. One hour later baseline data were recorded (the subjects did not woar the mask). Three hours after the first procedure, the control procedure was carried out.

Table 1: Time schedule

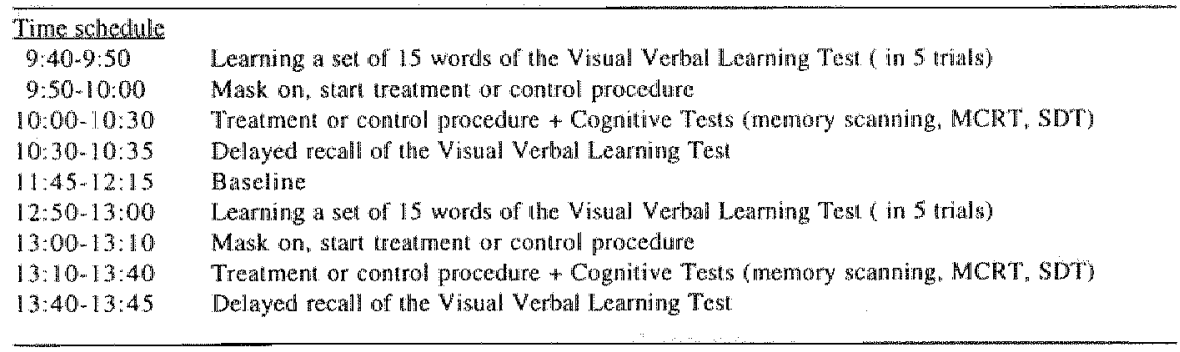




\section{Apparatus}

An infra-red gax analyzer (Datex Multicapde Multigas Monitor; CNO-103) was used to monitor end tidal $\mathrm{CO}_{2}$ during all treatments. The gas was sampled at a flow rate of $500 \mathrm{ml} / \mathrm{min}$ through a side tube of $190 \mathrm{~cm}$ length connected to a fully sealed oral nasal mask (Eros; F5341). Three $\mathrm{AgAgCl}$ electrodes (ArboBGmbH Medizn-Technologie) eonnected to a heart rate (HR) monitor (Datascope Passport Monitor) recorded the number of beats per minute (bpm). $\mathrm{SaO}_{2}$ was also measured continuously with a finger-oximeter (Datascopeb) connected to the oximeter. An experimenter recorded on paper $\mathrm{HR}_{3} \mathrm{SaO}_{2}$, and $\mathrm{CO}_{2}$ every 2 minutes. Respiration rate (RR) was recorded every minute as the number of inhalations. Average $\mathrm{CO}_{2}, \mathrm{SaO}_{2}, \mathrm{RR}$, and $\mathrm{HR}$ were calculated over the 30-minute testing period (table 3 ).

Treatments

Hypoxia (HO) was induced by inhalation of a gas mixture contained in a high pressure bottle filled with $13.6 \% \mathrm{O}_{2}$ and balanced with nitrogen. This mixture represents the oxygen/nitrogen composition of air at an altitude of $3500 \mathrm{~m}$ (or $11650 \mathrm{ft}$ ). A gas mixture containing normal air $\left(21 \% \mathrm{O}_{2}\right)$ was breathed through the mask as the control treatment (normoxia or $\mathrm{NO}$ ). $\mathrm{SaO}_{2}$ in the hypoxia condition was significantly different from the other inductions $(88.5 \%$ ) (table 3 ). Valves, operated manually, reduced the pressure from the cylinders to $5.52 \times 105 \mathrm{~N} \mathrm{~m} \mathrm{~m}^{2}$ for ambient level). The breathing mixture was connected to the oral-nasal mask via $190 \mathrm{~cm}$ of flexible, corrugated tubing with an inside diameter of $0.7 \mathrm{~cm}$.

For hypocapnic hyperventilation (HV) and its control procedure (normocapnic hyperventilation or NV) stubjects received normal air through the open mask. Hypocapnic hyperventilation was induced by increasing the breathing frequency of the subjects up to $50-70$ (on average 57.5) $\mathrm{imh} / \mathrm{min}$, until an end-tidal $\mathrm{CO}_{2}$ of on average $2.2(\mathrm{SD}=0.2) \mathrm{kPa}$ was reached. The experimenter instructed the subjects to "Breath in and out ats deep and fast as you can". For the control treatment (normocapnic hyperwentilation), the mask was connected to a tank containing $100 \%$ $\mathrm{CO}_{2}$. The pressure of $\mathrm{pCO}_{2}$ in the tubing connected to the open mask was low, 1.5 barr, and $\mathrm{pCO}_{2}$ was increased up to $5 \%$ (as compared to the percentage in normal air: $0.2-0.3 \%$ ) to compensate for the excess expired $\mathrm{CO}_{2}$. Thus, the lowered $\mathrm{pCO}_{2}$ in arterial blood seen in hypocapnic hyperventilation, was not seen during the control treatment, and arterial $\mathrm{CO}_{2}$ levels thus remained at nomal levels $\left( \pm 4.9 \mathrm{SD}=0.5\right.$ ). In hypocapnia and nomocapnia the $\mathrm{SaO}_{2}$ was somewhat higher as compared (98.8\%) to baseline $\mathrm{SaO}_{2}(97.9 \%$ ) (table 3). Recovery took place by instructing subjects to breath normally through the nose.

\section{Cognitive tests}

The cognitive assessments and dependent variables are listed in table 2. A distinction was made between primary and secondary variables, because we were mainly interested in the effect of treatments on the profile of cognitive functions, such as memory (working and long term), choice and incompatible choice RT, and visual sensitivity. The secondary outcome variables were measures of heart rate (as a measure of the stress perceived) and subjective ratings. The following thests were used.

\section{Primary cognitive measures}

Visual Verbal Learning Test with Distraction (Long term memory). One of six parallel lists of 15 monosyllabic meaningful nouns matched for word frequency, was presented on a computer screen every 2 seconds per word. The word was presented for $1 \mathrm{sec}$ and then the screen remained blank before another word was displayed (Brand \& Jolles, 1985). Simultaneously. 15 similar but different nouns were presented auditorily. For each measurement, a different visual word list and a different auditory list were used. List order was balanced over the experimental sessions to avoid 
learning effects or list type interference. Subjects were asked to verbally recall as many of the visually presented words as possible immediately after all words had been presented. This first immediate recall trial was followed by four more trials in which the same words were repeated in the same order. When the fifth trial was completed the subjects underwent the treatment which lasted 30 minutes. Thereafter, subjects were asked to name as many of the previously learned words as possible. This measure (delayed recall) was taken as at dependent variable to assess long term memory.

Memory Scanning Task (Working memory and sensorinotor speed). Subjects were briefly shown a set of letters and told to memorize them. This is called the "memory set" (Sternberg, 1969). Subjects then saw a series of 48 letters each displayed for $1000 \mathrm{msec}$ on a computer screen. After a further $2000 \mathrm{msec}$ the next letter appeared. Half of the presented letters were part of the memory set and had to be recognized as targets. The subjects pressed either 'YES' or "NO" to indicate whether or not each successive letter was one of those contained in the memory set. "The median of the response time (RT) for correctly identifying targets was taken as a dependent parameter (Brand \& Jolles, 1987). This task was performed with memory-sets consisting of 1, 2 , and 3 letters, respectively. Individual $R T$ set size functions were calculated, by using the equation $R T=A * S s+B$, in which: $A=$ slope of the function (i.e. the amount of extra time needed per item in the memory set), $\mathrm{Ss}=$ size of the memory set, and $\mathrm{B}=$ intercept with the $\mathrm{Y}$-axis. The slope is a measure of speed of scanning working memory, whereas the intercept measures sensorimotor speed (Sternberg, 1969).

The Motor Chotce Reaction Time Task. The subjects pressed a red button and were asked to press one of five other buttons when lit. The five buttons were lacated equidistantly from the held red button. Two time registrations per stimulus-response trail were measured, (1). initiation time (time needed to release the red button) and (2). movement time (itme needed to move from the red button to the target button). Two subtasks were used "compatible 3-choice RT' and "incompatible 3-choice RT". The response could consist of pressing one of three buttons that lit up ('compatible' 3-choice RT) or of pressing the button to the right of the lit button ('incompatible' 3-choice RT) as quickly as possible. In these two subtasks, the task factors, 'the number of response alternatives' and 'the degree of compatibility of the stimulus and the required response* were systematically varied within subjects (Houx, Vreeling, \&olles "1991).

Signal Detection Spatial Perception Task (Visual Sensitivity). In the visual search task (Klein. Ponds, Houx, \& Jolles, submitted), stimuli were presented in a pseudo-random order on a computer video monitor ( $640 \times 480$ pixtels resolution). Twenty stimuli were randomly assigned to a $10 \times 6$ grid, all stimuli being $2.5 \mathrm{~cm}$ apart. Stimuli were small squares of $3 \times 3 \mathrm{~mm}$; targets were defined as four stimuli forming a square of $2.5 \times 2.5 \mathrm{~cm}$. Every 1000 msec three stimuli were moved to a different location in the matrix. Subjects were seated $60 \mathrm{~cm}$ from the monitor and had to respond to targets with their dominant hand by pressing a button as quickly as possible. At the center axis the visual angle horizontally was 11.80; the visual angle vertically was 7.10. Thus, subjects had to move their eyes to be able to detect all targets. Subjects had to respond to targets within $2000 \mathrm{msec}$ after which targets disappeared. The total number of stimuli presented was 400 . With 56 targets, there was an overall signal probablity of 0.14 . The task lasted about 7 min. Targets were never consecutive stimuli. Seven targets were presented every $50 \mathrm{sec}$ and the same number of stimuli are presented in each quadrant of the inspection area. There was a practice session each time before the test had to be performed, which consisted of one block in which subjects were given feedback on their performance. According to the theory of signal detection (Pollack \& Norman, 1964), the proportion of correctly recognized (cr) signals allong with the proportion of falsely recognized (fr) signals constitute the non-parametric visual 
sensitivity measure: $A^{*}=(1-1 / 4) * f r / c r+(1-\mathrm{cr}) /(1-\mathrm{fr}) . \mathrm{A}^{*}$ is in fact the proportion of correctly recognized targets, corrected for the subject's response tendency. Because the distribution of $A^{\prime}$ is skewed due to a ceiling effect. $A^{\prime}$ was arcsin transformed before being used in the statistical analysis.

Taible 2: Tests used for cognitive assessments.

\begin{tabular}{|c|c|c|c|}
\hline Test & Concept & Dependent variables & Lnits \\
\hline \multicolumn{4}{|c|}{ 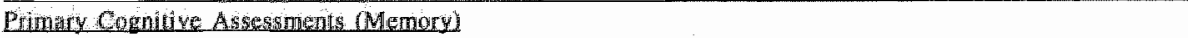 } \\
\hline WeT TINT & Long term memory & Delayed recall & of words \\
\hline Memory scarning & Speed of scanning working memory & Slope & mser \\
\hline \multicolumn{4}{|c|}{ 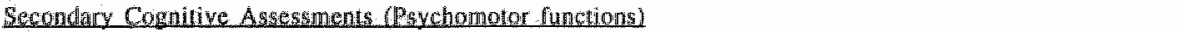 } \\
\hline Memory BCanning & Sensorinotor speed & 1 -intercept & misec \\
\hline \multirow{2}{*}{ Motor Choice RT } & Psychomotor speed. & 3-choice RT & msec \\
\hline & Response competition & Incompatible Choice RT & msec \\
\hline Signal Detection & Wisual sensitivity & $A^{\prime}($ corrected for FP) & $\%$ \\
\hline
\end{tabular}

\section{Secondary measures.}

Subjective ratings Before and after each task, subjects were asked to rate the discomfort experienced of a number of symptoms frequently known to occur during hypoxia and/or hypocapnic hyperventilation (Guyton, 1991) on a 0 (causes no discomfort) - 50\% (causes a little discomfort) - 100\% (causes a lot of discomfort) scalle. Symptoms listed were: nausea, cramps or pain in the chest, palpitations, trembling, feeling very hot, sweating, a tingling feeling in the fingers, shortness of breath, trouble breathing, headache, feelings of dereallisation, dizzyness, drowsiness, dry mouth.

\section{Statisties}

Baseline performance was subtracted from the performance during the control procedures, hypoxia and hypocapnia, to control for individual differences. Then, dependent variables were analyzed with repeated measures multivariate analyses of variance (MANOVA using SPSS 4.0 on Macintosh) according to a $2 \times 2$ factorial model. The between treatments factor had 2 levels (1) overbreathing (hypocapnia and normocapnia) versus (2) breathing through a mask (hypoxia and normoxia) assessed the effect of breathing frequency or overbreathing. The within treatments factor had 2 levels and assessed the effect of hypocapnia and hypoxia versus the control procedures (normocapnia and normoxia). The interaction effect signified whether there was a significant difference between the active treatments. Separate uniwariate analyses were performed to determine whether there were effects of wearing a mask (normoxia) and overbreathing (normocapnia) relative to baseline (BO and BV). This is a legitimate procedure if the comparisons are built into the design or are suggested by the theoretical basis of the experiment (Winer, 1971). (Probabilities of $5 \%$ or less were considered significant. Trend significant probabilities were set between 5 and $8 \%$ ). All analyses were performed with SPSS 4.0 on an Apple Macintosh computer. Results are displayed in table 3 
Table 3: Arterial $\mathrm{SaO}_{2}$ and $\mathrm{CO}_{2}$ during differem experimental conditions.

\begin{tabular}{|c|c|c|c|c|c|c|c|c|c|c|}
\hline Variable & Condition & $\mathbf{N}$ & Mean & SID & \multicolumn{3}{|c|}{ Multivariate } & \multicolumn{2}{|c|}{ Waiwarbale } & $p$ \\
\hline $\mathrm{SaO}_{2}$ & Baseline & 20 & 98.1 & (1.3) & 169.01 & $(2,18)$ & $<.001$ & & & \\
\hline \multirow[t]{4}{*}{$\left(\begin{array}{c}\text { 需 } \\
0\end{array}\right)$} & $\begin{array}{l}\text { Normoxia } \\
\text { Hypoxia }\end{array}$ & $\begin{array}{l}20 \\
20\end{array}$ & $\begin{array}{l}98.2 \\
88.5\end{array}$ & $\begin{array}{l}(1.0) \\
(2.6)\end{array}$ & & & & $\begin{array}{l}1.12 \\
350.5\end{array}$ & $\begin{array}{l}(1.19) \\
(1,19)\end{array}$ & $\begin{array}{l}\text {.ns i } \\
<.001\end{array}$ \\
\hline & Baseline & 20 & 97.9 & $(1,0)$ & 6.22 & $(2,18)$ & .01 & & & \\
\hline & Nommocapmia & 20 & 98.8 & $(0.7)$ & & & & 12.97 & $(1,19)$ & $<.001$ \\
\hline & Hypocapnia & 20 & 98.8 & $(0.9)$ & & & & .06 & $(1.19)$ &. $\mathrm{ns}$ \\
\hline $\mathrm{CO}_{2}$ & Normoxia & 20 & 5.0 & $(0.5)$ & & & & 1.28 & $(2,18)$ & $\mathrm{ms}^{3}$ \\
\hline \multirow[t]{3}{*}{$(\mathrm{KPa})$} & Hypoxia & 20 & 4.9 & $(0.5)$ & & & & & & \\
\hline & Normocapuia & 20 & 4.9 & $(0.5)$ & & & & 566.2 & $(1,19)$ & $<: 001$ \\
\hline & Hypocapnia & 20 & 2.2 & $(0.2)$ & & & & & & \\
\hline
\end{tabular}

1 between baseline and normoxia, ${ }^{2}$ between normoxila and hypoxia, ${ }^{3}$ Between normocapnia, normoxia and hypoxia,

4 Between mormocapnia and hypocapnia

\section{Results}

\section{Long term memory (Visual Verbal Learning Task)}

There was weak statistical support for a difference between treatments on the number of words recalled after $30 \mathrm{~min}$ (delayed recall) $[\mathrm{F}(1,19)=3.94, \mathrm{p}=.06]$, which was caused by the difference between normocapnia and hypoxia (table 4). There was no difference between the control procedures and the active treatments hypoxia or hypocapnia $[\mathrm{E}(1,19)=1.40 \mathrm{D}=\mathrm{ns}]$ and no interaction effect $[\underline{F}(1,19)=94, \underline{p}=\mathrm{ns}]$. Univariate analyses also revealed no difference between control procedures and baselines.

Working memory and sensorimotor speed (Sternberg memory scanning task)

There was no significant difference between treatments on the speed of search in working memory (slope) $[F(1,181)=.29, p=n s]$ nor was, there a significant effect within treatments $[\mathrm{F}(1,18)=1.54, \mathrm{p}=\mathrm{ns}]$ or an interaction effect $[\mathrm{E}(1,18)=38, \mathrm{p}=\mathrm{ns}]$. With respect to sensorimotor speed (1-intercept), there was no significant difference between treatments $[\mathbb{E}(1,18)=35, p=n s]$ of within treatments $[\mathrm{F}(1,18)=1.10, \mathrm{p}=\mathrm{ns}]$. The imteraction effect was also not significant $[\mathbb{E}(1,18)=1.97, \mathbb{2}=\mathrm{ns}]$

Separate univariate analyses revealed a larger slope during baseline performance as compared to normocapnic hyperventilation $[\mathbb{E}(1,19)=11.86, \mathbb{R}<005]$, which was caused by the slowing of the initial response times (1-intercept) during overbreathing $[E(1,19)=[8,48, \mathbb{2}<.001]$. Hence, overbreathing slowed subjects considerably, since no difference was detected between hypocapnia and normocapnia. Wearing a mask also slowed subjects $[\mathbb{E}(1,19)=23.24,[2<.001]$ to a similar extent as overbreathing [intercept: $\mathrm{E}(1,19)=2.62, \mathrm{p}=\mathrm{ns}$ ]. In addition, there was a trend for hypoxia to induce additional slowing as compared to normoxia on the 1 -intercept $[\mathbb{E}(1,19)=3.78, \mathbb{p}=.07]$ but not when compared to overbreathing. The slopes were not different for baseline, normoxiat and hypoxia.

"Due to appartus failure, data of one subject were missing on the memory scanning task during the hypocapnic hyperventilation induction 


\section{Choice and Jncompatible Choice Response Time (Motor Choice Reaction Rime task)}

The choice intiation response time (RT) was significantly slower during overbreathing (hypocapnid and normocapnia) as compared to wearing a mask (normoxia and hypoxia) since there was a significant difference between treatments $[\mathrm{F}(1,19)=24.12, p<.0001]$ but no significant difference between control and active treatrments $[\mathrm{F}(1,19)=16, \mathrm{p}=\mathrm{ns}]$, and no significant interaction effect $[\mathrm{E}(1,19)=05, \mathrm{p}=\mathrm{n} 8]$. Separate univariate analyses on the choice initiation time task rewealled ignificant differences between baseline and between wearing a mask $[F(1,19)=31.05, p<.0001]$ and between baseline and overbreathing $[F(1,19)=52.55, p<.0001]$.

For the incompatible choice RT task, the initiation time showed no difference between $[\mathrm{F}(1,19)=$ 2.35, $p=n s]$ or within treatments $[E(1,19)=71, p=n \&]$, but did reveal a significant interaction effect $[\mathrm{E}(1,19)=6.60, \mathrm{p}<05]$. Separate univariate analyses showed that, while performance when wearing a mask (normoxia) was not different from baseline $[\mathbb{E}(1,19)=1.20, \mathrm{p}=n \mathrm{~s}]$, the initiation $\mathrm{RT}$ of the incompatible task was significantly lower during normoxia as compared to hypoxia $[E(1,19)=8.81, \mathfrak{p}<.01]$. No difference was seen between hypocapnia and normocapnia $[\mathbb{E}(1,19)=1.10, \mathrm{D}=\mathrm{ns}]$. Furthermore, no differences existed between the effects of overbreating (hypocapnia and normocapnia) and hypoxia on this subtask. The movement time for both subtasks was not different between or within treatments (data not shown).

Visuall Perceptual Sensitiwity (Signal Detection Test)

There was no difference between treatments $[\mathrm{E}(1,19)=01, \mathrm{p}=\mathrm{ns}]$, but there was a trend for a difference between active treatment and controls $[F(1,19)=3.68, p=.07]$. No interaction effect was seen $[E(1,19)=.03, \mathrm{D}=\mathrm{ns}]$. Separate univariate analyses revealed, while performance while wearing a mask (normoxia) was not different from baseline $[F(1,19)=48, p=n s]$, there was a significant effect of hypoxia $[\mathbb{E}(1,19)=7.18, \mathrm{p}<02]$ as compared to wearing a mask. However, the awerage visual sensitivity during hypocapnic hyperventilation was low and not different from the effect of hypoxia on the visual perceptual sensitivity performance $[\mathrm{E}(1,19)=.00, \mathrm{p}=\mathrm{ns}]$. Yet, due to a larger variance in the hypocapnic condition, no difference between the two overbreathing conditions was detected $[\mathbb{E}(1,19)=1.21, p=n s]$. Furthermore, no difference was seen between wearing a mask and overbreathing $[\mathrm{E}(1,19)=.07, \mathrm{p}=\mathrm{ns}]$.

Table 4. : Entries are mean (SD) of the delayed recall (LTM) of the Visual Verbal Learning test (VVLT), sensorimotor speed (intercept) wnd working (slope) memory of the Sternbere memory scanning lesi (MEMSCAN), intiation Chotoe RT (CRT) and Incompatible Choice RT (ICRT) of the Motor Choice Reaction time Test (MCRT), and vistal perceptual sensitivity (A* corect adjusted for false positives) of the Signal Detection Tent (SDT) over conchitions

\begin{tabular}{|c|c|c|c|c|c|c|}
\hline Parumeter & Dasetine & Normoxia & Hypoxia & Baseline & Normocapnia & Hypocapnia \\
\hline \multicolumn{7}{|l|}{ VLLT (LTM) } \\
\hline \# of words & $11.5(2.5)$ & $110(4.2)$ & $10.9(3.6)$ & $10.60(3.0)$ & $11.7(1.8)$ & $10.5(3.0)$ \\
\hline \multicolumn{7}{|l|}{ MEMSCAN } \\
\hline intercept (nnse) & $37 ! \llbracket 46\rceil$ & $397(41)$ & $418 \quad(62)$ & 371 (34) & $412(46)$ & $409(51)$ \\
\hline slope (msec) & $46(16)$ & $36 \quad(27)$ & $38 \quad(19)$ & $48 \quad(20)$ & 33 (19) & $43 \quad(30)$ \\
\hline \multicolumn{7}{|l|}{ MCRT } \\
\hline CRT (msee) & $3,33(38)$ & $363(42)$ & $359 \quad(49)$ & $333(32)$ & $393(50)$ & $391(61)$ \\
\hline ICRT (nnsec) & $411(56)$ & 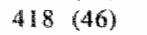 & $438(60)$ & $402(48)$ & $432(52)$ & $423(47)$ \\
\hline \multicolumn{7}{|l|}{ SDT } \\
\hline$A^{*}(w)$ & $98 \quad(02)$ & (02) & $(02)$ & $98 \quad(02)$ & (02) & $96(03)$ \\
\hline
\end{tabular}


Secondary variables - Heart rate. Respiration rate, and Subjective Assessments

Table 5 lists the effects of treatments on heart rate (HR) as an indicator of induced stress and the discomfort (number and severity of symptoms) experienced. Hypoxia that a stronger effect on HR than normoxia, although normoxia also had a small yet significant effect on HR as compared to baseline. Hypocapnic and nomocapnic hyperventilation had the strongest effects on HR. The effect of hypoxia on HR was smaller than the effect of normocapnic hyperventilation, HR during NV was \pm 76 bpm and during $H O$ it was $\pm 70 \mathrm{bpm}[\mathrm{F}(1,19)=14.03, \mathrm{p}<.001]$.

Respiration rate (RR) was not different between hypoxia (on average $15 \mathrm{inh} / \mathrm{min}$ ) and nomoxia $[\underline{F}(1,16)=0,16, \underline{p}=\mathrm{ns}]$. $R R$ was was significantly lower during hypoxia as compared to during hypocapnic hyperventilation $[E(1,16)=223.04, p<0001]$ but was not different betiveen hypocapnia and normocapnia $\left[\mathrm{E}\left(1,16^{3}\right)=3.7, \mathrm{~g}=\mathrm{ns}\right]$.

Hypoxia and normoxia gave rise to a similar number of symptoms. However, subjects reported more symptoms after normoxia than at baseline (table 5). Most symptoms were reported after hypocapnic hyperventilation. However, the discomfort experienced by symptoms was similar between hypocapnic hyperventilation and normocapnic hyperventilation (on average $41 \%$ ). Hypoxia gave rise to fewer symptoms and less discomfort than hypocapnis isympt: $\mathrm{E}(1,18)=35.8, \mathrm{p}<.001 ; \mathrm{HO}<\mathrm{HV}, \mathrm{E}(\mathbb{1}, 18)=4.6, \mathrm{p}=.05]$. Several subjects also reported headache and drowsiness at baseline. However, in general, hypocapnic hyperventilation and normocapnic hyperventilation gave rise to a wide range of symptoms whereas hypoxia and normoxia gave rise only to a limited number of symptoms.

Table 5: Heart rate and symptoms (number of and bother experienced by themj during the expermentat condinions

\begin{tabular}{|c|c|c|c|c|c|c|c|c|c|c|}
\hline Nariable & Condition & $\mathbf{N}$ & Mean & SD & \multicolumn{3}{|c|}{ Multivariate } & \multicolumn{2}{|c|}{ Univariate } & $\mathbf{p}$ \\
\hline HR & Baseline & 20 & 63.1 & $(10.2)$ & 17.18 & $(2,18)$ & $<.001$ & & & \\
\hline \multirow[t]{5}{*}{ (bpm) } & Normoxia & 20 & 66.4 & $(10.5)$ & & & & 9.53 & $(1,19)$ & $<01$ \\
\hline & Hypoxia & 20 & 70.0 & $(10.2)$ & & & & 9.02 & $(1,19)$ & $<.01$ \\
\hline & Baseline & 20 & 64.1 & $(10.0)$ & 30.69 & $(2,18)$ & $<.001$ & & & \\
\hline & Normocapnia & 20 & 76.2 & $(10.5)$ & & & & 57.24 & $(1,19)$ & $<001$ \\
\hline & Hypocapnia & 20 & 74.4 & $(11.7)$ & & & & 1.07 & $(1,19)$ & $\mathrm{fus}$ \\
\hline \multicolumn{11}{|c|}{ Af of symptoms reported } \\
\hline \multirow[t]{6}{*}{$(0.15)$} & Bascline & 20 & 0.6 & $(0.8)$ & 13.43 & $(2,18)$ & $<.001$ & & & \\
\hline & Mornoxia & 20 & 2,4 & $(2.0)$ & & & & 32.26 & $(1.19)$ & $<.0101$ \\
\hline & Hypoxia & 20 & 27 & $(1.8)$ & & & & .52 & $(1.19)$ & $10 \mathrm{~s}$ \\
\hline & Bastine & 20 & 0.6 & $(0.7)$ & 31.24 & $(2,18)$ & $<.001$ & & & \\
\hline & Nonmocapnia & 20 & 5.3 & $(3.0)$ & & & & 49.93 & $(1.19)$ & $<.00 \mid$ \\
\hline & Hypocaptia & 20 & 6.9 & $(3,9)$ & & & & 5.79 & $(1,19)$ & $<.05$ \\
\hline \multicolumn{11}{|c|}{ s: bother experienced by symptoms } \\
\hline \multirow{6}{*}{$(0-100)$} & Baseline & 20 & 13.3 & $(19.7)$ & $9: 98$ & $(2,18)$ & $<.001$ & & & \\
\hline & Mornoxia & 20 & 25.3 & $(15,6)$ & & & & 4.60 & $(1,19)$ & .05 \\
\hline & Hypoxia & 20 & 32.8 & $(18.2)$ & & & & 2.88 & $(1,19)$ & nits \\
\hline & Baseline & 20 & 9.0 & $(11.0)$ & 29.84 & $(2,18)$ & $<001$ & & & \\
\hline & Normocapnia & 20 & 40.3 & $(18.4)$ & & & & 35.67 & $(1,19)$ & $<.001$ \\
\hline & Hypocapnia & 20 & 42.5 & $(19.6)$ & & & & .13 & $(1,19)$ & $n: s$ \\
\hline
\end{tabular}




\section{Discussion}

We compared the hypoxia model and the hypocapnic hyperventilation model as experimental models for cognitive deficiency. $\mathrm{SaO}_{2}$ and $\mathrm{CO}_{2}$ levels were monitored to ensure the correct induction of hypoxia and hypocapnic hyperventilation. It has been suggested that the cognitive effects of hypoxia are mediated by compensatory hypocapnic byperventilation which would result in vasoconstriction of the cerebral arteries (Fowler et al, 1985; Noble et al, 1993). However, we did not detect compensatory hyperventilation ( $\mathrm{RR}$ and $\mathrm{CO}_{2}$ ) in our subjects during hypoxia.

As for the cognitive functions, neither hypocapnic hyperventilation nor milld hypoxia affected working and long term memory, as assessed with a memory scanning task and a verbal learning task, respectively. It is possible that our memory tests were not sensitive to the effects of brain lhypoxia, since earlier studies did find an effect of hypocapnic hyperventilation on working memory (Kraaier, 1988; Rother, 1994). However, these studies did not employ control procedures. This is important as our findings show that hypocapnic hyperventilation did not differ from the control procedure, normocapnic hyperventilation. Furthermore, effects of mild hypoxia on memory have been reported (Denison et al, 1966) but several other studies were also unable to detect an effect of mild hypoxia on memory functions (Fowler et al, 1985; 1994). Fowler et al (1985) mentioned that methodological aspects (a combination of hypoxia, exercise and hypocapnic hyperventilation) of the earluer studies may cast doubt on the observed effect of mild hypoxia on memory functions. Similar to our findings, Fowler et al (1994) reported that mild hypoxian did not affect memory functions. First, they found that performance of a dichotic listening task revealed no increase in the rate at which unattended digits were forgotten. The authors concluded that no storage deficit could be found with this task. This is comparable to the absence of treatment effects on the delayed recall of the verbal learning task (i.e. memory consolidation) in the present study. They also found a decrease in the intercept, but not in the speed of search in working memory (slope) of a memory scanning task based upon the Sternberg paradigm. Fowler et al argued that the effects of mild hypoxia can be interpreted either as an impairement of the early stages of the processing of sensory stimuli or alternatively as an impairement of executive dysfunctions.

In this line, as expected, we found that hypoxia decreased the visual sensitivity of the signal detection lask as compared to normoxia. Although some authors mention an increase in visual sensitivity during mild hypoxia (Schlaepfer, Bartsch, \& Fisch, 1992), in general, hypoxia is thought to negatively affect wisual sensitivity. In fact, this effect has long been known as a "dulling of the senses" (Haldane, Kellas, \& Kennaway, 1919). Whether this effect is a central "lethargy" effect, which already occurs at $2970-3500 \mathrm{~m}$ in altitude (Forester, 1985), or a peripheral effect (Fowler et al, 1987: Gibson, Pulsinelli, Blass, \& Duffy, 1981) is unclear. For instance, the slowing of response times has been attributed to a decreased peripheral visual sensitivity to brightness discrimination (Fowler et al, 1987). However, Kida and Imai (1993) showed that the response times increased in association with the N2P3 ERP components, reflecting a central impairement of sensory discrimination and evaluation processes. Furthemore, Noble et al. (1993) reported negative effects of hypoxia on reaction time tasks, inclependent of visual sensitivity. We also found that mild hypoxia as compared to normoxia increased the RT for the most difficult task, the incompatible $\mathbb{R T}$ task requiring inhibition. These findings are consistent with the hypothesis of Fowler et al. (1994) that mild hypoxia may slow the central excutive function which reduces the capacity to process complex information. This is reflected in the slowing of functions, which is seen, similarly to aging, especially on more complex psychomotor tasks (visual detection, inhibition of response) under the pressure of time (Huppert, 1991: Salmasso, 1993). 
Unexpectedly, no effects of hypocapnic hyperventilation as compared to its control procedure were found. It is possible that the effects of overbreathing can be attributed to the dual task paradigm (Nideffer, 1979). Subjects had to breath in a regular fast rhythm and were also distracted by central and peripheral symptoms while doing the main task, which may have slowed performance.

However, overbreathing induced a similar slowing of response times on the incompatible choice $\mathrm{RT}$ and a decrease in visual sensitivity as hypoxia. First, the effects of hypoxia were small but were actual effects of low oxygen as they were significant when compared to nomoxia. Yet, wearing a mask also had some detrimental effects on the speed of response time on the 1-intercept and the choice RT task. These effects may be attributed to purely mechanical aspects of the mask (tightness of the mask), or indirectly to the stress (increase in HR) associated with wearing a mask, which may have interfered with concentration. Furthermore, effects of overbreathing were not due to vasoconstrictive effects, since these effects are caused by a lowering of the pH which could not occur during nomocapnia. Also, in a pilot study, we found a rapid compensatory vasodilatory response, measured with doppler chromotography techniques, which took place within 2-5 min after hypocapnic hyperventilation was started and which lasted for at least $20 \mathrm{~min}$. However, after 30 min of hypocapnic hyperventilation, a low average visual sensitivity on the last task, the SDT was detected, but these effects were not significant due to a larger variation in performance. It thus remains possible that the compensatory vasodilatory effects of hypocapnic hyperventilation are reversed after some time.

In surm, mild hypoxia was seen to have small effects on the visual sensitivity and the incompatible RT test when compared to normoxia. However, overbreathing largely had similar effects and wearing a mask was allso seen, to a lesser extent, decrease the speed of response times. This indicates that future studies which investigate the effects of experimental drug interventions in the hypoxia modlel or the hypocapnic hyperventilation model should include appropriate control procedures. 


\title{
Chapter 6
}

\section{Cognitive Performance After Strenuous Physical Excercise}

\author{
Eef Hogervorst", Wim Riedel ", Asker Jeukendrup ${ }^{2}$ \& Jelle Jolles \\ 'Department of Psychiatry and Neuropsychology. University of Limburg Masasticht, the Nethetlands. \\ 2 Department of Human Biology, University of Limburg, Mastricht, the Netherlinds.
}

Publishedi in: Perceptual and Motor Skills, 1996, 83, $479-488$

\begin{abstract}
Stimulating as well as detrimental effects of exercise on cognitive functioning have been reported. In the present study, 15 endurance-trained athletes (aged 18 to 42 years) performed a bicycle ergometer endurance test at $75 \%$ of their maximal work capacity (Wmax). Psychomotor and cognitive tests were administered before and immediately after exercise. These consisted of simple reaction time (RT), 3-choice RT and Stimulus-Response (S-R) incompatible RT tasks, a finger tapping task, and the Stroop test. Simple RT tasks, but also the S-R incompatible RT and the Color Word Interference in the Stroop test showed an increase in speed of performance after exercise compared to baseline. An enhanced activation was probably responsible for this better performance on psychomotor and cognitive tests. Since performance in the most complex task, the Interference subtest of the Stroop, was also improved after exercise, the expectancy of the subjects of a potential positive effect of exercise may have been responsible.
\end{abstract}

\section{Introduction}

Controversies exist over the effect of exercise on cognition. Several studies have described a negative effect of strenuous physical exercise on performance of cognitive tasks (Gutin, 1973; Isaacs \& Pohlman, 1991; Hancock \& McNaughton, 1986; McMorris \& Keen, 1994; Salmela \& Ndoye, 1986). Fatigue has often been hypothesized to underlie the negative influence of intense exercise on cognition (Tomporowski, Ellis, Stephens, 1987) but not all authors have found strenuous exercise to have a negative effect on cognitive functions (Adam, Teeken, Y pelaur Verstappen, in press; Bard \& Fleury, 1987; Côte. Salmela, \& Papathanasopoulu, 1992; Fleury, Bard, Jobin, \& Carriere, 1981; Paas \& Adam, 1991; Tomporowski et al., 1987). The various results are difficult to compare. Some studies used subjects from different backgrounds and with different levels of fitness, and different cognitive tasks were used either during or after exercise of a varying duration (McMorris \& Keen, 1994; Tomporowski \& Ellis, 1986). Often, exercise of a short duration (varying from 2 to 15 minj was used and the level of fatigue was not evaluated. Matters are further complicated since as Tomporowski and Ellis (1986) stated there is no agreement on the definition of fatigue.

According to Tatakuwa (1971) fatigue is defined as the combined output of mental activity and physiological functions. This definition was used by Hancock and McNaughton (1986), who found that fatiguing exercise (running on a treadmill at or above the anaerobic threshold level) inhibited especially higher-level mental skills such as decision making. Isaacs and Pohlman (1991) also showed that exercise with a heavy workload (cycling at 100\% of the maximal oxygen 
uptake ( $\mathrm{VO}_{2} \mathrm{max}$ ) as compared 1075,40 and $25 \%$ and rest) negatively affected performance on coincidence anticipation task which measures skilled timing performance. These studies supported the conclusions of Fleury, Bard and Carriere (1981) that fatigue mainly affects complex cognitive functioning that requires a great deall of the resources of the central nervous system. However, Paas and Adam (1991) found an unexpected beneficial effect of a substantial change in workload (cycling at 75.85\% of the $\mathrm{VO}_{2} \mathrm{max}$ ) on a decisional task. No effects were found on a simpler perception task. It is thus unclear whether and how physical fatigue affects simple and complex cognitive processing.

A possible flaw of former studies is the use of short duration exercise $(<15$ min) to induce fatigue. Thus, in the present study, subjects were tested before and immediately after prolonged ( $\pm 60 \mathrm{~min}$ ) endurance exercise. Subjects were not tested during exercise since earlier McMorris and Keen (1994) and Isaacs and Pohlman (1991) hypothesized that divided attentional mechanisms rather than actual effects of fatigue caused the decreased performance which was seen during cycling on cognitive tasks. At higher levels of arousal, subjects may focuse rather on internal perceptions of discomfort (a theory first described by Nideffer, 1979), than focuse on cognitive tasks. Endurance tests are thought to induce exhaustion or physical fatigue (Jeukendrup. Saris, Brouns, \& Kester, 1996) which is then implicated in the decline in performance that occurs in any prolonged or repeated task (Kennedy, 1988). The present study is similar to the study by Fleury et al. (1981) in that fatigue is defined as the consequence of a physical work load on subsequent psychological performance. If fatigue affects the functioning of the central nervous system, fatiguing exercise should especially affect complex, decisional, cognitive tasks (Easterbrook 1959; Gutin, 1973; Kennedy. 1988; Fleury et al., 1981). Because subjects can compensate for a lack of energy by investing additional effort in a psychological task (Zijlstra \& Meijman, 1989), the effort invested in task performance was taken into account as a modulating variablle. Simple measures of speed (tapping and simple reaction times) were also taken into account. Performance of these tasks often improves after or during exercise, possibly due to enhanced activation (Easterbrook, 1959; Gutin, 1973; Salmela \& Ndoye, 1986). Heart rate was thus measured as an indication of the intensity of performance and the level of arousal or activation at the time of testing. We tested the hypotheses that (1) subjects would perform faster on simple tasks after an endurance test as compared to before the test and (2) subjects would perform worse on complex cognitive tasks after an endurance test.

\section{Method}

\section{Subjects}

Fifteen healthy male triathletes and competitive cyclists participated in the study. The subjects had enrolled in a validation study of endurance performance (Jeukendrup et al., 1996). They trained on a regular basis ( $22 \mathrm{~h} / \mathrm{day}$ and $z 4$ times/week). The mean age of the subjects was $24.9 \mathrm{yr}$. (7.9, range 18-42), their nean weight was $73.5 \mathrm{~kg}(6.5)$ and their mean height was $183 \mathrm{~cm}(4.5)$. Design and Procedure

The experiment was conducted according to a single-factor repeated-measures design. The factor "Level of exercise" consisted of three levels (pre-exercise, post-exercise, baseline). Subjects came to the laboratory three times at 7-day intervals. The first visit was intended to familiarize the subjects with the tests and procedures.

On the second visit, heart rate and cognitive tests were measured before and after the endurance test. Baseline measurements were taken on the third visit. 
One week before the experiment, maximall workload was attained during an incremental exencise test until exhaustion on a bicyle ergometer. After a warm-up period of $5 \mathrm{~min}$ at $100 \mathrm{~W}$. the workload was inereased every $2.5 \mathrm{~min}$ until heart rate reached 160 beats/min. Then worklowd was increased with $25 \mathrm{~W}$ every $2.5 \mathrm{~min}$ until the pedaling rate dropped below $60 \mathrm{rmm}$. The maximall work capacity $(W \max )$ was determined by the following formulla: $\mathrm{Wmax}=\mathrm{Wout}+(\mathrm{V} / \mathrm{150}) 25$ where Wout is the workload of the last completed step, $t$ is the time in the final step. Mean maximal workload of the subjects was $385 \mathrm{~W}(33)$, indicating that subjects were well-trained.

The exercise consisted of a short warmup ( 5 min $100 \mathrm{~W}$ ) followed by a sinulated time trial. In this time trial subjects were asked to perform a certain amount of work (equal to about 1 hour cycling) as fast as possible. This total amount of work was based on the maximal workload (Wmax) according to the formula:

\section{Total amoun of work $=0.75 *$ Wmax 3600}

The ergometer was set in the linear mode according to the formula

$$
W=L \cdot(R P M)^{2}
$$

in which $R P M$ is the pedaling rate and $\mathrm{L}$ is a linear factor. This factor was choosen in a way which would canse a pedaling rate of 90 RPM at $70 \%$ of the maximal work capacity. In other words the linear factor was dependent on a subjects' maximal work capacity. This would mean that $75 \%$ of the maximal work capacity could be achieved at about 100 RPM which appeared to be the preferential pedaling rate of most cyclists.

This type of exercise can be classified as 'maximal' or 'exhaustive' and subjects usually report maximall wallues on scales of perceived exertion (Salmela \& Ndoye, 1986).

\section{Apparatus}

During the experiment, the subjects cycled on a Lode electronically braked ergometer (Lode. Excaliber Sport $\mathrm{R}$, Lode BV, Groningen The Netherlands). Heart rate was measured electronically using a dinamapR (Critikon, type 8100 ) before and immediately after exercise.

Psychomotor and cognitive tasks

Subjects completed a short test battery (15 min) which included complex cognitive and psychomator tests. The following tests were included. The Stroop Color-Word Test is a well-known test for the ease of shifting perceptual sets to conform to changing task requirements (Lezak, 1983). The test consists typically of three subtests (Bohnen, Jolles, \&. Twijnstra, 1992). In subtest 1,10 rows by 10 colums of color names (red, blue, green, and yellow) are printed in black on white cardboard. In subtest $\mathrm{II}_{n}$ the same number of correspondingly colored patches are printed, whereas subtest III contains a number of color names, printed in incongruously colored ink. For instance the word "red" can be printed in green. The protocol for admistering the test in this study was as follows. For subtest I ("Color Word Reading"), the subject was requested to "read the color names row by row, as fast as you can, without making any mistakes". The time needed to complete the wholle card was recorded with a stopwatch. For the second subtest ("Color Naming") the instruction was to "name the colored patches". The third subtest (Color-Word Interference") involved naming the color of the ink the color words were printed in, without paying attention to the word itself. For each subtest the time taken to finish the card was recorded. In this study the short version was used. The short version stops after 40 stimuli (whereas the whole test contains 100 stimuli). The correlations between the abridged wersion and the original version is remarkably high (Pearson's $r= \pm .93, p<.001$ on average for all cards) (Klein, Ponds, Houx, \& Jolles, submitted).

The Motor Choice Reaction Time Test. The subjects pressed one button and were asked to press one of five other buttons located equidistantly from the hold button when they lit up. This yielded reaction times in three subsequent conditions of increasing task complexity. The response 
set consisted of pressing only one button that lit up (simple RT), pressing one of three buttons that lit up(3-choice RT) or pressing the button to the right of the lighted button (incompatible 3choice RT) as guicklly as possible. Hence, in these three subtasks, two task factors are systemetically varied within subjects: the number of response alternatives, and the degree of compatibility of the stimulus and the required response (Houx, Vreeling, \& Jolles, 1991). The Finger Tapping Tesi. Finger tapping speed is one of the more simple aspects of psychomotor performance. The time elapsed between two single taps was registered with an IBM (type 486) compatible computer at millisecond accuracy. In this experiment the number of taps per second (on a response button on a panel connected to the computer), using the preferred hand, was used as a dependent wariable (Brand \& Jolles, 1987).

Effort. Effort was measured using the Rating Scale for subjectively experienced Mental Effort.

("Beoordelingsschaal Mentale Inspanning"). Apart from a global indication of the psychophysiological state of the subjects (fatigue), the amount of effort can also be seen as an indicator of the "cost" of performing a task. This scale was constructed by using the "magnitude estimation method" with several different population samples (bus drivers, students) and different rating modalities to estimate the scale values of the nine adjectives (not at all exhausting, hardly exhausting, slightly exhausting to extremely exhausting) (Zijlstra \& Meijman, 1989). The data from the original $15-\mathrm{cm}$ visual analog scale were transformed to percentages.

\section{Statistics}

The "Level of" exercise" effect was tested for each task according to a repeated-measures design with three levels: low (pre-exercise), high (post- exercise), and low (baseline). The main effect of Level of exercise" was evaluated in conjunction with task factors in repeated measures within subjects analysis of variance. The task factors were: number of response alternatives (2 levels: 1 or 3 responses); and S-R Compatibility (2 levels: Compatible, Incompatible) in the Motor Choice Reaction Task; and Color Naming ( 2 levels: Words, Colors); and Color-Word Interference (2 levels: Response Conflict, No Response Conflict) in the Stroop task. Post hoc paired ti-tests were used to determine the exact nature of the differences found between the pairs of experimental conditions. Probabilities of $5 \%$ or less were considered significant. Significant probabilities for trends were between 5 and $10 \%$. All analyses were performed with SPSS 4.0 .

\section{Results}

Heart rate was significantly different over conditions as multivariate analyses showed $[E(2,13)=36.65, p<.001]$. Heart rate was significantly lower before exercise $[(14)=-8.72, p<.001]$ and baseline $[t(1.4)=8.71, p<.001]$ as compared to after (see Table 1).

The subjects took significantly longer to complete the Stroop subtask Color Naming than they did the subtask Color Word Reading. Multiwariate analysis revealed a main effect of stimululus type (Words, Colors) $[E(1,14)=57.88$, p $<.001]$. Also a main effect of the factor "Level of exercise" $[\mathrm{F}(2,13)=15.97, \mathrm{p}<001]$ and a significant interaction between stimulus type and "Level of exercise" were detected $[\mathrm{F}(2,13)=5.78, \mathrm{p}=.02]$. Post hoc paired $\mathrm{t}$-tests between conditions revealed learning effects on both cards. Time needed to perform Color Word Reading was significantly longer before as compared to after exercise $[t(14)=2.36, p<.05]$ and baseline $[t(14)=2.74, p<.05]$ and equal after exercise as compared to baseline $[t(14)=-.48, n . s$.$] , signifying$ a bottom effect. On Color Naming the learning effect was even more clearly shown, since significantly more time was needed to perform this task before exercise as compared to after 
$[t(14)=3.17, p<01]$ and baseline $[t(14)=495, p<001]$ and after exercise as compared to baseline $[t(14)=2.79, p=.01]$. Response time on the Color-Word Interference subtask was significantly longer than that on the Color Narning subtask since multivariate analysis showed that a main effect of stimulus type (Response Conflict, No Response Conflict) was significant $[E(1,14)=$ $52.79, \mathbb{D}<.001]$. Also, the "Level of exercise" effect $[\mathrm{E}(2,13)=7.14, \mathbb{2}<.01]$ and the interaction effect between stimulus type and "Level of exercise" were shown to be significant $[\mathbb{E}(2,13)$ $=10.34, p<01]$. As can be seen in Table 1 , exercise clearly affected Color-Word Interference. This was confirmed with post hoc paired t-tests. Time needed to perform the Color-Word Interference was longer before exercise as compared to after $[t(14)=3.42, p<.005]$ but equal to baseline $[t(14)=1.58, p>10]$. The baseline performance was significantly longer than the time needed to perform the task after exercise $[t(14)=-3.56, p<005]$.

Table 1: Means (SD) of HR (beats/min), the Stroop (time naded to finish the cards in sec), the Motor Choice Reaction Time (MCRT: mean Reaction Time (RT) in msec, SRT: Simple RT, CRT: Choice RT, and ICR: Incompatible RT), Finger Tapping Test (FlT: the number of taps per sec), and Effort (a 0 - 100 scale, 0 not exhausting, 100: extremely exhausting pre-exercise, post exercise and at baseline.

\begin{tabular}{|c|c|c|c|c|c|c|}
\hline \multirow[b]{2}{*}{ Task } & \multicolumn{2}{|c|}{ Pre-exercise } & \multirow{2}{*}{$\begin{array}{l}\text { Post } \\
\text { Mean }\end{array}$} & \multirow{2}{*}{$\begin{array}{r}\text { arencise } \\
\text { SD }\end{array}$} & \multicolumn{2}{|c|}{ Baseline } \\
\hline & Mean & $\mathrm{SD}$ & & & Menn & SD \\
\hline Hetrt rate (bpm) & 63.1 & 15.3 & 103.0 & 14.5 & 65.5 & 14.6 \\
\hline \multicolumn{7}{|l|}{ Suroop test } \\
\hline Color Reading & 13.3 & 2.0 & 12.6 & 20 & 12.7 & 2.1 \\
\hline Color Naming & 17.6 & 3.5 & 16.5 & 2.6 & 15.5 & 2.5 \\
\hline Color-Word Int. & 23.3 & 6.3 & 20.8 & 4.4 & 22.2 & 4.6 \\
\hline \multirow{4}{*}{$\begin{aligned} & \text { MotorChoice. } \text { SimpleRT } \\
& \text { ChoiceRT } \\
& \text { Imwomp RT }\end{aligned}$} & 378 & 37 & 369 & 48 & 379 & 51 \\
\hline & 429 & 53 & 417 & 39 & 420 & 43 \\
\hline & 494 & 61 & 485 & 65 & 488 & 64 \\
\hline & 7.0 & .8 & 6.9 & .6 & 7.0 & .8 \\
\hline Effort score & 53.1 & 3.9 & 54.5 & 4.8 & 51.2 & 4.3 \\
\hline
\end{tabular}

On the Motor Choice Reaction Time test, Choice RTs were shown to be longer than simple RTs. Multivariate analysis revealed a main effect of the number of response alternatives (2 levels: 1 or 3 responses) $[\mathrm{E}(1,13)=113.36, \mathrm{p}<.001]$. A main effect of the factor "Level of exercise" was seen on multivariate analysis $[E(2,12)=6.28, p=01]$. Exercise did not affect the relation between the number of response alternatives and RT, since no interaction was seen between the number of response alternatives and "Level of exercise" $[E(2,12)=.7 \rrbracket, n . s$.]. Post hoc paired $t$-tests showed that a trend significant lower response time on the simple reaction time task was seen after exercise as compared to before exercise $[1(14)=1.99, \mathrm{p}=07]$. No difference was seen between the pre-exercise condition and baseline $[t(131)=-.17, n s]$ but response time after exercise was significantly lower as compared to baseline $[\mathrm{t}(13)=-2.81, \mathrm{p}=.01]$. Choice reaction time did not show any effect of condition, response time was equal over conditions. Incompatible RTs took longer than compatible RTs. Multivariate analysis revealed a main effect of " $\mathrm{S}-\mathrm{R}$ Compatibility" (2 levels: Compatible, Incompatible) $[E(1,13)=57.32, \mathbb{R}<.01]$. A non significant trend effect of "Level of exercise" $\llbracket E(2,12)=2.87, \mathrm{p}=09]$ emerged, indicating a possible effect of exercise in the Incompatible task. The interaction between "S- $R$ response Compatibility" and "Level of exencise" was not significant, however $[\mathrm{E}(2,12)=05$, n.s.]. On the incompatible subtask, a trend

\footnotetext{
1 Data of one of the subjects was missing diue to apparatus failure
} 
significant smaller response time after exercise as compared to before $[\mathrm{t}(14)=1.91, \mathrm{p}=.08]$ was seen. However, no differences were seen when pre- and postexercise conditions were compared with baseline conditions ( $p>50$ ). The average number of taps per second was 6.97 and did not differ significantly between conditions $[\mathrm{F}(2,1)=18$, n.s.]. The average subjectively experienced effort was 52.90 on a scale of 100 and did not significantly differ between conditions $[\mathrm{F}(2,13)=17, \mathrm{n} . \mathrm{s}$.

\section{Discussion}

The results of the experiment support the first hypothesis, namely that exercise has a positive effect on performanice speed in simple tasks. Simple RTs were significantly lower after fatiguing exercise than at baseline. However, simple manual motor speed, which is mainly limited by peripheral factors (Tapping), was not influenced at all by high intensity exercise. Furthermore, the results did not support the second hypothesis, that endurance exercise would have a general detrimental effect on complex cognitive functions. In fact, the opposite was found. For instance, the RT for the most complex task that required the inhibition of a learned response, the ColorWord Interference subtask of the Stroop test, was decreased by endurance exercise. Important is that the degree of subjectively experienced effort was not increased, nor were more errors made with the higher speed of performance.

The findings seem to indicate that there is no clear-cut relation between physical fatigue immediately after endurance exercise and mental fatigue as was suggested by Tatakuwa (1971). However, it is possible that the subjects were not thoroughly fatigued, and it may be worthwhile to study fatigue after an extended period of endurance exercise.

The enhanced performance on psychomotor and cognitive tasks can be explained in the following fashion. Gutin (1973) proposed that various levels of activation differently affect certain types of mental tasks. Low levels of activation are best for performance of tasks which require a great deal of information processing. Low levels of activation are reflected by a slight increase in heart rate during and after exercise $(90-120 \mathrm{bpm})$. Higher levels of activation, provoked by long duration exercise ( $> \pm 15 \mathrm{~min}$, inducing a heart rate of more than $160 \mathrm{bpm}$ ) should diminish performance on tasks that require a great deal of information processing. In the study of Isaacs and Pohlman (1991) in which exercise had a negative effect on complex tasks heart rate was $150-175 \mathrm{bpm}$. Although we found that longer duration exercise at heart rates of 160-180 bpm had a beneficial effect on performance of complex cognitive tasks, these effects werte seen after exercise, which was contrary to Gutin's expectations. However, during the actual testing the level of activation was still sufficiently enhanced (heart rate $90-120 \mathrm{bpm}$ ) to lead to an improved performance consistent with the first hypothesis. Thus, at the time of psychomotor and cognitive testing, the subjects were still in a highly aroused state. Hence, the enhanced performance on the tasks after exercise might reflect a generally increased activation of the central nervous system. The abscence of this effect, as reflected by the results on the Tapping task , could be due to a ceiling effect.

The finding of a significant interaction between exercise and the results of the most complex task, the Stroop Color-Word Interference is interesting. This effect may be comparable to that found by Paas and Adam (1991) who also reported a unexpected beneficial effect on a decisional task during a substantial change in workload (cycling at $75-85 \%$ of the $\mathrm{VO}_{2} \mathrm{max}$ ). The authors concluded that subjects in their study apparently had decided to invest more processing capacity or resources into the more complex (decision) task. Similar findings were reported by Adam et al., (in press). Although effort in our study was not elevated after excercise, it is possible that 
subjective effort scales are not reliable or valid indicators of the actual effort invested. An (unjconscious decision made by the subjects to actually invest more effort in certain tasks is influenced by motivational and probably the effects of expectancy. Tomporowski and Ellis (1986) already suggested that many studies may have been confounded by expectancy effects. The expectancy of the participants influences performance through motivational variables. Hence, the subjects may have compensated to overcome the possible effects of fatigue. This compensation effect is especially seen in highly trained subjects and is not mediated by levels of fitness, but rather, as mentioned by Tomporowski and Ellis (1986), by the expectancy that exercise has a beneficial effect in general. Subjects who do not often engage in high intensity exercise often have a negative expectancy about the effect of exercise on cognition. For instance, Delignières, Brisswalter \& Legros (1994) showed that experts exerted additional effort to overcome the effects of fatigue when challenged. Their performance increased as exertion increased (cycling at $20,40,60$, and $80 \%$ of maximal aerobic power). Non- experts with a similar level of fitness showed a decrease in performance on their 2 and 4 choice RT task. The error rate in both groups remained stable.

It is thus unclear whether the effects seen in the present study are mediated by the effects of activational and/or expectancy. However, it is clear that expectancy variables and valid measures of effort should be taken into account in studies into the effects of fatigue. Preferably, expectancy should be controlled while effort is measured in an experimental design to study the actual relation between fatigue, expectancy, and effort. 


\title{
PART II : CAFFEINE AND COGNITION
}

\section{Chapter 7}

\section{Caffeine improves Memory Performance during Distraction in Middle-Aged, but not in Young or Old Subjects.}

\author{
E. Hogervorst, W. J. Riedel, J. A. J. Schmitt, \& J. Jolles
}

Accepted Dec, 24, 1997 Human Psychopharmacology

\begin{abstract}
The present study evaluated the effect of caffeine $(225 \mathrm{mg})$ on cognitive performance in young, middle-aged, and old subjects in a placebo-controlled parallel groups design $(n=60)$. Groups were matched for level of education and sex. Positive effects of caffeine, as compared to placebo, were found in middle-aged subjects in the first trial of the word learning test. In contrast, caffeine had negative effects on the speed of searching working memory in young subjects. Caffeine had no effect on the intercept, which is an indicator for sensorimotor speed, of a memory scanning task. The middle-aged subjects appeared to regularly consume twice as much caffeine as the young and old subjects. These results were similar to earlier findings in a large population study. Although statistical analyses with habitual caffeine consumption as a covartate did mot yield different results, a caffeine withdrawal effect was hypothesized to be responsible for the reduced cognitive performance of middle-aged subjects receiving placebo. The habitual use of large amounts of caffeine by middle-aged subjects may be a means to overcome the age-related decrease in cognitive functioning that is caused by changes in information processing
\end{abstract}

\section{Introduction}

Caffeine is thought to act as a central stimulant and to have effects on cognitive functioning by enhancing alertness and vigilance (Goldstein \& Kaizer, 1969; Lieberman, Wurtman, Einde, Roberts, \& Coviella, 1987). Although it has been reported that caffeine has no direct effects on memory functions (Loke, 1988), it was recently found that $250 \mathrm{mg}$ of caffeine could attenuate scopolamine-induced memory dysfunction without increasing psychomotor speed (Riedel, Hogerworst ,Leboux, Verhey, van Praag, \& Jolles, 1995). This is of interest for studies of cognitive aging, in view of the decrease in memory functioning with age and the development of cognition enhancers.

The present study evaluated the effects of caffeine on memory and memory-related processes in a cross-sectional study involving three age groups (young, middleaged, and old). Effects of caffeine in middle-aged subjects are especially interesting because of known differences in habitual caffeine use between cohorts (Riedel, Hogervorst, \& Jolles, 1995) and because cognitive performance has been found to be diminished in the fourth and fifth decades of life (Houx, 
Vreeling, \& Jolles, 1991; Jolles, Houx, wan Bottel, \& Ponds, 1995). Moreover, most studies generally compare young and old subjects. There have been relatively few studies of the effect of caffeine in ageing subjects.

In a large population study of 7414 subjects, improved performance was associated with higher levels of caffeine consumption. Elderly subjects appeared more susceptiblie to the performance improwing effects of caffeine on memory, visuospatial reasoning, and reaction time tasks (Jarvis, 1993). Yu, Maskray, Jackson, Swift and Tiplady (1991) found that the performance of elderly subjects on a continuous attention test improved with caffeine relative to placebo. The elderly subjects also felt more alert, energetic, and interested with caffeine. Another study (Swift \& Tiplady, 1988) reported that young and old subjects responded differently to caffeine. The authors suggested that the beneficial effects of caffeine in elderly subjects were possibly due to central mechanisms, as reflected by a decrease in the number of errors on cognitive tasks. The beneficial effects were not mediated by an increase in psychomotor speed, as they were in the younger subjects. Lorist, Snel, Mulder, and Kok (1995) however, did not find substantial differences between the effects of caffeine on cognitive functions in elderly and young subjects. They found that caffeine could only partly counteract the decreased energetical resources of elderly subjects during task performance, in that caffeine counteracted the age-related performance decrement related to perceptual processes. However, caffeine had a similar beneficial effect in young, well rested subjects on stimulus evaluation. Caffeine did not affect central processes, i.e. the speed of searching working memory.

It is thus currently unclear whether caffeine has a different effect in young or old subjects and at which levels of information processing caffeine acts. In the present study, the effect of $225 \mathrm{mg}$ of caffeine on sensorimotor and memory functions was investigated in young, middle-aged, and old subjects. Extreme care was taken to include only healthy subjects in view of the studies of Houx and Jolles that health-related factors influence neurocognitive performance in otherwise healthy subjects (Houx, Vreeling, \& Jolles, 1991; Houx \& Jolles, 1993; Jollles, Verhey, Riedel., \& Houx, 1995). Because we were mainly interested in the performance of middle-aged subjects, we used a newly developed word learning test with distraction in which subjects had to ignore distracting auditory information. This test was used because it is potentially more sensitive for cletecting subtle effects in middle-aged subjects as compared to the young subjects. We expected caffeine to have different effects in the three age groups.

\section{Method}

\section{Subjects}

Sixty healthy subjects were recruited by means of an advertisement in a local newspaper. The mean age of the young group $(n=20)$ was 29.4 (range: $26-34$ years), the mean age of the middle uged group ( $n=20$ ) was 49.6 (range: $46-54$ years), and the mean age of the eldest subjects ( $n=20$ ) was 69.1 (range: 66-74 years). Subjects were pre-selected over the telephone. Only subjects without any previous adverse health-related factors were included (Houx et al, 1991; 1993). Factors screened for were treatment by a neurologist / neurological disorders (e.g. cerebrovascular attacks, epilepsy, Parkinson's Disease, Multiple Sclerosis, or any other muscle or nerve disease, severe head trauma, encephalitis, or meningitis); cardiovascular disease (e.g. hypertension, arrythmia); internal disease e.g: cancer, diabetes, thyroid disease, and renal dialysis); treatment by a psychiatrist and/or the presence of severe psychiatric disturbances (e.g. depression and electroshock treatment); the use of medication liable to induce cognitive deficits; and the excessive use of alcohol ( $>40$ consumptions/ week) or the use of addictive drugs. Lastly, 
subjects who had sensory and/or motor impaiments which could be expected to affect psychomotor performance were excluded. Groups were matched for sex and level of occupational ability (LOA). Each group contained 10 males and 10 females. The LOA may be a better estimator of intelligence than education, especially in older subjects. For this purpose; occupation was given a four-digit code, based on a detailed functional description (CBS, 1985) that was transformed to a 7 -point score, which ranged from 1 (simple labour requiring no education or a low level of skills) to 7 (highly specialized labour at an academic level) (van den Brandt, Goldbohm, van't Veer, Volovics, Hermus, Sturmans, 1985). The LOA was derived from the Directorate General for Employment Policies in the Netherlands (1989). Each group contained subjects with 10 low and 10 high LOAs, the average LOA was 45. Subjeots were asked how much coffee or tea they consumed a day. Caffeine consumption for each age group is shown in fig 1.

\section{Procedure}

The study was a double blind, placebo controlled design, matched for sex and LOA. Groups were order balanced for time of testing to avoid the influence of circadian factors. All testing was done in the morning. The entire procedure took 1.5 hours. Subjects were asked not to drink any alcohol containing beverages 24 hours preceding testing procedures and to arrive at the laboratory well rested. Subjects were asked not to drink any beverages containing caffeine or related substances, such as theophylline (coffee, chocolate, tea, or coca cola) on the morning of testing. On arrival in the laboratory, a saliva sample was collected and subjects were instructed to drink $225 \mathrm{mg}$ of caffeine in a total of 3 cups of coffee in $15 \mathrm{~min}$. For the placebo condition, 225 mg of decaffeinated coffee was used.

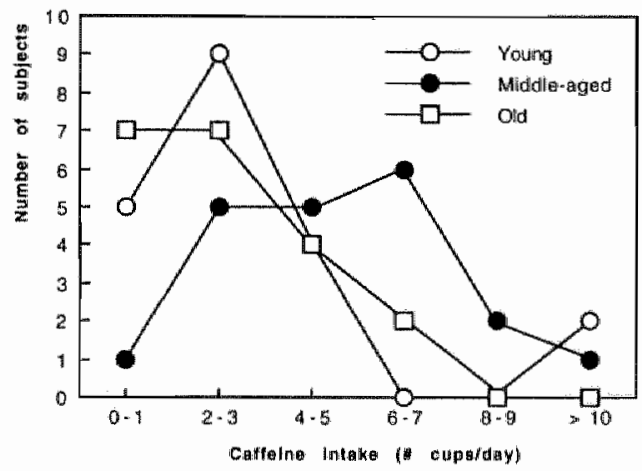

Fig.1. Caffeine intake in cupsiday in young, middleaged and old subjects

After half an hour there was a 15-min training session. Thereafter, subjects started the test procedure, which took approximately $30 \mathrm{~min}$. Subjects were seated in front of an IBM compatible computer in a sound proof room. Standardized instructions were given through an intercom. After the experiment, subjects rinsed their mouth with water and a saliva sample was collected.

\section{Saliva Caffeine analysis}

Saliva samples were taken before and after (abowt 75 min after caffeine consumption) experimental sessions. Samples were stored at $-220 \mathrm{C}$. Saliva caffeine concentration was analysed using a high-performance liquid chromatography method. 


\section{Deychologicallesing}

Two cognitive tests that are sensitiwe to the effects of aging were used. Visual Verbal Learning Test with Auditory Distrackion (VVLT-D). One of tweive parallel hists of 15 monosyllabic meaningful nouns, matched acröss word frequency, was presented on a computer screen connected to an IBM compatible computer at a rate of 2 seconds per word (Brand \& Jolles, 1985 ). Simultaneously, 15 similai but different nouns were yresented auditorily. Subjects were asked to verbally recall as many of the visually presented words as possible after the presentation had stopped, ignoring the (distracting) auditory words. The first triall was followed by four more trials in which the same words were repeated in the same order. The number of correctly recalled words was noted after each trial. The furst trail was taken as an index of short term memory. The trial with the maximum number of recalled words is also taken as an dependent variable (immediate recall). Twenty min after completion of the fifth trial, subjects were asked to recall as many of the previously learned words that were presented visually as possible (clelayed recall or long term memory). Memory Sicanning. Subjects were briefly shown a set of unrelated consonants and told to memorize them. This is called the "memory set" (Sternberg, 1969 ; Brand \& Jolles, 1988). Subjects then looked at as series of 48 letters displayed for $1000 \mathrm{msec}$ on a computer screen, connected to an IBM compatible computer; after $2000 \mathrm{msec}$ the next letter appeared. Half of the presented letters were part of the memory set and had to be recognized as targets. The subjects' task was to respond as rapidly as possible by pressing either 'YES' or 'NO' to indicate whether or not each successive letter was one of those from the memory set. The median of the response time (RT) for correct responses (on targets and non targets) was taken as a dependent parameter. This task was performed with memory sets consisting of 1,2 , and 3 letters, respectively. Individual RT $\mathrm{x}$ set size functions were calculated. This function is defined by the equation $R T=A * S s+B$, in which: $A=$ slope of the function (i.e. the amount of extra time needed per item in the memory set), $S s=$ size of the memory set, and $\mathrm{B}=$ intercept with the $\mathrm{Y}$-axis. The slope is a measure of the speed of scanning working memory, and the intercept is a measure of sensorimotor speed.

\section{Statistics}

The results were analysed using a two-way analysis of variance (ANOVA) with the factor Drug (caffeine, placebo) and the factor Age (young, middle-aged, old). For habitual caffeine use nonparametric analyses (resp. Kruskal-Wallis and Manth-Whitney runs test) were carried out. All analyses were done with the SPSS-PC statistics package for Apple MacIntosh. The cognitive test performance of the various groups is given in table 1 .

Table 1 : Qutcome (mean and stdev) of test performance measures in young $(n=20)$ and midde-aged (n=17), and alderly sutbjects $(n=20)$ with caffeine $(n=10)$ or placebo $(n=10), \quad(n=9, n: n=8)$

\begin{tabular}{|c|c|c|c|c|c|c|c|c|c|c|}
\hline \multirow[t]{2}{*}{ Test } & \multirow[t]{2}{*}{ Measturia } & \multicolumn{2}{|c|}{ roung } & \multicolumn{3}{|c|}{ Middle-aged } & \multicolumn{4}{|c|}{ Old } \\
\hline & & Calfeine & Placebo & \multicolumn{2}{|c|}{ Caffeline } & Placebo & \multicolumn{2}{|c|}{ Caffeine } & \multicolumn{2}{|c|}{ Placebon } \\
\hline \multicolumn{11}{|l|}{ VVLIT } \\
\hline Trial 1 & (A of wonds) & $5,0 \quad(1,7)$ & $6.0(1.5)$ & 5.3 & $(1.1) *$ & $4.0(1,1)^{\wedge}$ & 4.3 & $(1.8)$ & 4. 1 & $(1.2)$ \\
\hline Maximmed reo & (\# of words) & $12.6(1.3)$ & $12.9(1.1)$ & 12.2 & $(1.7)^{*}$ & $11.5(1.2)^{n}$ & 11.4 & $(1.7)$ & 11.3 & $(1.7)$ \\
\hline Delayed recall & (H of words) & $10.6(1,5)$ & $11.3(1.8)$ & 9.8 & $(1.8)^{*}$ & $9.0(3.0)^{n}$ & 8.7 & $(2.8)$ & 9.8 & $(2.4)$ \\
\hline \multicolumn{11}{|c|}{ Memory scaunimg } \\
\hline Intarcept & (utinsec) & $(47)$ & $433(38) *$ & 466 & $(40)^{n}$ & $455(46)^{\mathrm{A}}$ & 510 & $(72)$ & 502 & $(71)$ \\
\hline Slope & (mnsec) & $(15)^{\prime}$ & $38 \quad(17)^{m}$ & 41 & $(15)^{\mathrm{m}}$ & $53 \quad(17)^{\mathrm{A}}$ & 47 & (15) & 57 & $(18)$ \\
\hline
\end{tabular}




\section{Results}

\section{Saliwa caffeime concentrations}

Three (1 caffeme, 2 placebo) subjects in the middle-aged group had pretreatment saliva caffeine concentrations slightly over $2.0 \mathrm{mg} / \mathrm{L}$, which suggested that they had not adhered to theabstinenee instructions. Data for these subjects, who had an average habitual caffeine use, were not ased in the analyses. The overall average pretreatment saliva caffeine concentration in the remaining: subjects was $.34 \mathrm{mg} / \mathrm{L}(\mathrm{SD}=.44)$, which was not different between conditions [CAF:M=.37 (SD.45), PLAC:M=31 (SD.43), $\mathrm{E}(1,54)=32, \mathrm{p}=\mathrm{ns}$ ]. Saliva caffeine concentrations were higher in the subjects that had received caffeinewcontaining coffee $(M=3.12 \mathrm{mg} / \mathrm{L}, \mathrm{SD}=1.47)$ than the subjects who hadreceived decaffeinated coffee $(\mathrm{M}=.33 \mathrm{mg} / \mathrm{L}, \mathrm{SD}=.46)[\mathrm{E}(1,54)=93.2, \mathrm{p}<.0001]$. Performance

A 2 (drug) $x 3$ (age) analysis of variance of performance on the VVLT-D revealed a main effect of age on the number of words recalled during the first trial $[\mathrm{E}(2,51)=4.10, \mathrm{n}<.05]$. There was no significant main effect of caffeine $[E(1,51)=0.09, \mathrm{~g}=\mathrm{ns}]$. There was a trend significant interaction effect of age and caffeine on trial $][E(2,51)=2.99, \mathrm{p}=.06]$. Figure 2 shows that the middle-aged subjects receiving placebo stored less words in short-term memory than did the subjects receiving caffeine.

There was a significant main effect of age on the maximum number of words recalled over the 5 trials $[E(2,51)=4.57, p<05]$ (table 1). Caffeine had no main effect on the maximum number of words recalled $[E(1,51)=0.14, \mathrm{p}=n . \mathrm{s}$. $]$. Also, no interaction effects were seen between age and caffeine on the maximum number of words recalled $[\mathrm{E}(2,51)=0.55, \mathrm{p}=\mathrm{ns}]$. There was a significant main effect of age on the delayed recall $[\mathrm{F}(2,51)=3.32, \mathrm{p}<.05]$ (table 1). Caffeine did not have a main effect on delayed recall $[\mathrm{E}(1,51)=0.44, \mathrm{p}=\mathrm{ns}]$ and also no interaction effect between age $x$ caffeine could be detected $[E(2,51)=0.85, p=n s]$.

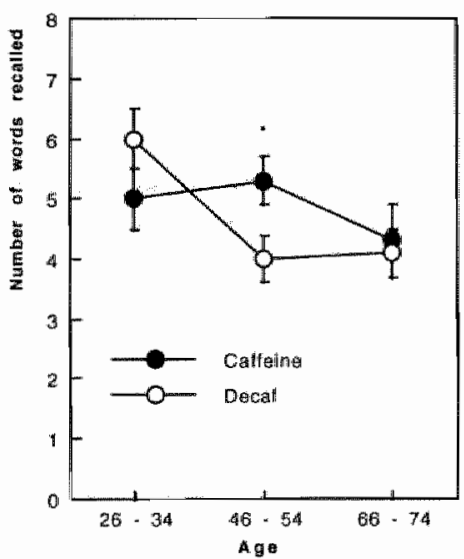

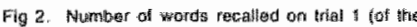

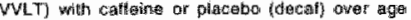

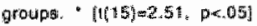

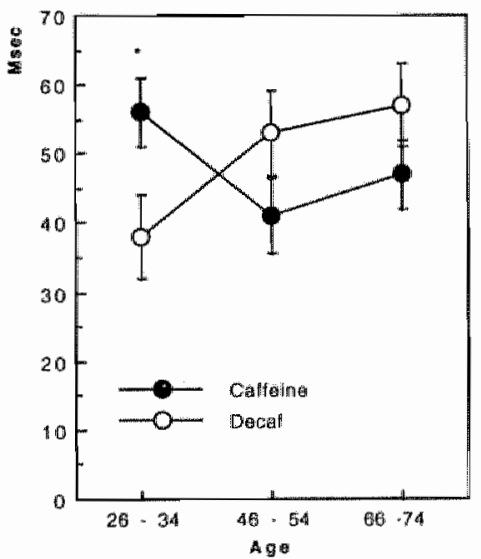

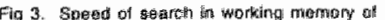

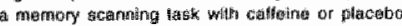

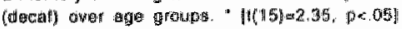


No main effect of age $[E(2,472)=0.53, \mathrm{p}=\mathrm{ns}]$ was seen on the slope of the Memory Scanning Task. Hence, age did not affect the speed of searehing working memory. In addition, no main effect of caffeine was detected $[\mathbb{E}(1,47)=0.12, \mathrm{~g}=\mathrm{ns}]$. However, there was a significant interaction effect between age and caffeine on the slope $[\mathrm{F}(2,47)=4.58, \mathrm{p}<0.05]$. Figure 3 shows that in the young group, the slope wass significantly lower for the subjects who received placebo than for the subjects who received caffeine. Age cllearly affected sensorimotor speed, as a 3 (age) $\times 2$. (caf/placebo) ANOWA on the 1-intercept showed $[\mathrm{F}(2,49)=9.67, \mathrm{p}<001]$. However, caffeine did not affect sensorimotor speed $[\mathrm{E}(1,49)=0,05, \mathrm{p}=\mathrm{ns}]$ and no interaction effect of age and caffeine was detected $[\mathrm{E}(2,49)=0.15, \mathrm{p}=\mathrm{ns}]$.

\section{Habizual use}

The three age groups differed in the average amount of caffeine consumed per day $[\mathrm{Chi}(57)=10.89, \mathrm{R}<.005]$. Young subjects consumed 3.2 (median: $2.8, \mathrm{SD}=2.6$ ) cups of coffee/ day, middle-aged subjects consumed 5.4 (median: $5.3, \mathrm{SD}=2.8$ ) cups of cofee/day, and old subjects consumed 2.6 (median: $2.1, \mathrm{SD}=2.0$ ) cups of coffee/day. The mean amount of caffeine was similar for the young and old subjects ( $2-3$ cups of coffee/day, $Z=-69, \mathrm{p}=\mathrm{ns}$ ) and significantly lower than that of the middle-aged subjects (vs young: $\underline{Z}=-2.60, \mathbb{p}<.01$; old: $\underline{Z}=3.06$, D<.005).

\section{Discussion}

The objective of the present study was to evaluate the effects of caffeine on cognitive functions in three age groups: young, middle-aged, and old healthy subjects. The results show that caffeine had selective effects on cognitive performance in the three age groups. First, as expected, age affected performance in the verbal learning test with distraction and also clearly affected sensorimotor speed, as measured from the intercept of the memory scanning task. Age did not affect the speed of searching working memory, as measured from the slope of the memory scanning task. Furthermore, young subjects who drank caffeine-containing coffee needed significantly more time to search working memory than did young subjects who drank decaffeinated coffee (placebo). By contrast, in the middle-aged subjects, caffeine had a positive effect on short-term memory or working memory storage capacity, as measured with the first trial of the word list task. More words were retained in memory after consumption of caffeine than after consumption of decaffeinated coffee. Caffeine did not affect maximum and delayed recall of the word list task, or the sensorimotor speed parameters of a memory scanning task.

There are various factors which may explain the different effects of caffeine on short term or working memory functions in the three age groups. In the first place, since the pharmacokinetics of caffeine do not change with age (Blanchard \& Sawers, 1982), the difference in the response to caffeine in the different age groups is thought to have a pharmacodynamic basis. However, there wax a difference in habitual use of caffeine in the three age groups. The middle-aged subjects drank almost twice as much coffee a day as the young and old subjects. The groups were not a priori matched for habitual caffeine consumption in order to conform to standard procedures in cross-sectional studies in cognitive aging research, where caffeine use is not a matching criterion.

2 Four subjects (3 young. 1 middle-iged) were not included in the analyses of the sllope, since these data were missing due to computer failure. Two subjects (one young, one middle-aged) were missing in the intercept data. 
Similar cohort differences in the regular use of caffeime have been reported earlier (Riedet, Hogerworst, Jolles, 1995), where young subjeets were seen to drink on average 3 cups of coffee 7 day, middle aged 5 cups, and old subjects 3,5 cups. Cohorl differences in the vise of nicotine have also been found (Hogervorst, Riedel, \& Jolles, 1995). These differences are usually controlled by statistical procedures. We also performed statistical analyses with the number of cups of coffee consumed per day as a covariate. The results remained essentially the same and f:values remained in the same order. This can be taken to indicate that inter subject differences in habitual caffeine consumption were not the major determinant of differences in the effects of caffeine found in the present study. Yet, habitual caffeine use has been shown to affect the outcome of tasks which measure memory or memory-related processes. Loke (1988), for example, found that regular caffeine users $(388-928 \mathrm{mg} / \mathrm{wweek}$ ) recalled more words after a delay than very low caffeine ( $6178 \mathrm{mg}$ ) users. Likewise, Smith, Rafferty, Lindgren, Smith, and Nespor (1991) reported that moderate to high habitual users of cafleine ( $>200 \mathrm{mg} / \mathrm{day}$ ) had increased sensitivity on a vigilance task. This may explain the absence of effects in our low caffeine consuming elderly subjects.

Further, a high habitual use may also render subjects more sensitive to caffeine withdrawal effects. This could be a second explanation for the findings of the present study. However, the effect of caffeine withdrawal on cognitive functions is disputed (James, 1994). The performance of habifual caffeine consumers on simple tasks is reported to decrease during caffeine deprivation (Silverman, Evans, Strain, \& Griffiths, 1992). Furthermore, in coffee-drinking managers (Streufert, Pogash, Miller, Gingrich, Landis, Lonardi, Severs, \& Roache, 1995) cognitive effectiveness during complex task performance was diminished during caffeine deprivation. Lieberman, Wurtman, Emde, Roberts, \& Coviella (1987) showed that even very low doses of caffeine ( $32 \mathrm{mg}$ ) enhanced cognitive function (vigilance and RT) after caffeine abstinence for 12 h. Some dispute whether caffeine deprivation in habitual caffeine consumers is the only explanation for the enhancing effect of caffeine on cognitive functions (Rogers, Richardson, \& Dernoncourt, 1995; Warburton, 1995). In the study performed by Warburton (1995) lihe effect of low doses of caffeine ( 75 and $150 \mathrm{mg}$ ) was investigated after a minimal caffeine abstinence of 1 h. Attention, problem solving , and delayed recall were improved but immediate recall and shortterm memory were not. Howewer, performance in the placebo condition was close to the maximum, giving little margin for improvement. Hence, even without caffeine deprivation, subjects in the caffeine condition still improved their cognitive performance. Yet, in view of the fact that our middle-aged subjects used nearly twice as much caffeine as the young and the old subjects, the effect of caffeine withdrawal was probably much more pronounced in the middleaged subjects than in the other subjects. This is interesting because it may provide chess as to the mechanisms of action of changes in cognition in relation to age. Young subjects, for example, have an optimal cognitive performance and it is possible that middle-aged subjects use coffee to compensate for the deterioration of cognitive functions with age. Also, many studies have shown that a decline in cognitive performance is already evident in the fourth decade (Jolles et al, 1995; Houx et al, 1991; 1993). This hypothesis is currently being tested in the Mastricht Aging Sudy, which is a large scale longitudinal study involving 1980 subjects aged 25 to 80 (Jolles et al. 1995).

The effect of caffeine taken together with the deterioration of information processing resources with age may explain the differences in caffeine/age effects in the present study. Salthouse (1988) suggested that the age-related decrease in short-term or working memory capacity reflects the decrease in resources available with advancing age. According to the model of Hasher \& Zacks (1979), sensitivity to difficult tasks which require effortful information processing, increases with 
age because these tasks require attention and energetic capacity, or resources. Moreover, elderly subjects are more sensitive to distrating information and find it difficult to actively suppress irrelevant information (Carlson, Hasher, Zacks, \& Connelly, 1995; Kane, Hasher, Stoltzfus, Zacks, \& Connelly, 1994). Caffeine positively affects the ability to focus attention (Lorist, Snel, Kok, and Mulder, 1994; Lorist, Snel, Mulder, \& Kok, 1995). The model of Hasher \& Zacks combined with the positive effect that caffeine has on selective attention may explain why caffeine had an effect on performance in the first trial of the word hist in middle-aged subjects, but not in young subjects. Caffeime increased the number of words recalled by reducing the susceptibility to the distracting, auditorily presented words, but only in the middle-aged subjects because these subjects are more sensitive to distraction and will profit from the effect of caffeine on selective or focused attention. The more structural deficits (loss of neural connections) of elderly subjects may explain the abscence of positive effects of caffeine in this age-group. A third explanation of our findings thus inwolves the known effects of caffeine on attentional functions (Lorist, Snel, Kok, \& Mulder, 1994; Nehlig, Daval, \& Debry, 1992). Similarly to our finding, others have reported negative effects of caffeine on the slope of a memory scanning task performed by young subjects (Anderson, Revelle, \& Lynch, 1989). However, caffeine has also been reported to have significant positive effects on sensorimotor speed (intercept) (Anderson, Revelle, \& Lynch, 1989; Lorist, Snel, Kok, and Mulder, 1994; Lorist, Snel, Mulder, \& Kok, 1995). The absence of intercept effects in the present study may be because our task was of short duration and caffeine is known to increase the ability to sustain attention or to maintain vigilance (Nehlig, Daval, \& Debry, 1992; Pons, Trenque, Bielecki, Moulin, \& Potier, 1987; Yu, Maskray, Jackson, Swift \& Tiplady, 1991). Furthermore, as for the effects of caffeine on other aspects of information processing, in the present study caffeine did not affect maximum and delayed recall. Hence, these data suggest that caffeine does not affect long term storage and consolidation functions.

In sum, age-related changes in information processing may thus underlie the effects of caffeine on cognitive performance. It remains to be established whether there is a dose-dependent relationship in this respect. There is convincing evidence that in young subjects caffeine does not affect performance in a linear dose-dependent manner. Rather, the dose-response relationship follows an inverted $U$-shaped curve analogous to the relation of performance and arousall which is described by the Yerkes-Dodson law (1908). For instance, Loke (1988) reported in her literature review that memory span increases with low (e.g. $162 \mathrm{mg}$ ) but not with high $(325 \mathrm{mg}$ ) doses of caffeine. In the present study, $225 \mathrm{mg}$ of caffeine had a negative effect on working memory performance of young subjects, but did not affect delayed recall. Anderson et al (1989), who showed a negative effect of caffeine on working memory functions, used a comparable dose of 4 $\mathrm{mg} / \mathrm{kg}$ caffeine. Interestingly, delayed recall has been shown to increase with $200 \mathrm{mg}$ but to decrease with $400 \mathrm{mg}$ of caffeine, while $325 \mathrm{mg}$ had no effect (see also: Smith, Kendrick, \& Maben, 1992; Terry \& Phifer, 1986). It may be the case that, because arousal decreases with age, the Yerkes-Dodson curve moves to a position more to the right. This would explain why caffeine had negative effects in young subjects, positive effects in our middle-aged subjects and no effect in the elderly subjects.

Concluding, results show that age has subtle effects on memory functions under conditions of distraction. In addition, caffeine has different effects on cognitive performance in different agegroups. The decreased performance in the middle-aged subjects who drank decaffeinated coffee may be due to a caffeine withdrawal effect, which is stronger than in subjects of other ages because the habitual caffeine consumption in middle-aged subjects was twice as high. The present findings are important for cognitive aging research in view of the fact that cross-sectional studies usually do not control for caffeine use. In addition, the neurocognitive paradigm used 
(measurement of memory performance under distraction) may prove relevant to cognitive aging research. The habitual use of large amounts of caffeine by middle-aged subjects may be a means to overcome the age-related decrease in cognitive functioning that is catused by changes in information processing.

\section{Acknowledgement}

The caffeine saliva sample analyses were made possible by a grant from the Institute for Scientific Information on Coffee, Paris 


\title{
Chapter 8.
}

\section{Caffeine decreases memory performance in stressful experimental conditions}

E. Hogervorst, W. Riedel, P. Hameleers, J.Schmitt, S. Seij, N. Rozendaal, M. v. Boxtel, P.J. Visser, \& J. Jolles

\begin{abstract}
The present study evaluates the effects of caffeine on cognitive performance in two models of experimentally induced cognitive dysfunction. Recently, caffeine was reported to attenuate the scopolamine induced cognitive dysfunction (Riedel et al, 1995). We report here effects of caffeine on the cognitive dysfunction induced by hypoxia and hyperventilation.

Twenty healthy male college students (aged 23-30) were tested according to a mixed, double blind, placebo-controlled cross-over design. Caffeine (230 mg) or placebo was administered according to a between subjects design. On one day, subjects received hypoxic air $\left(13.6 \% \mathrm{O}_{2}\right)$ and normal air ( $21 \% \mathrm{O}_{2}$, normoxia) through a mask as a control. On another day, with one week in between, they hyperventilated (end tidal $\mathrm{CO}_{2}<2.5 \mathrm{kPa}$ ) and hyperventilated with additional $\mathrm{CO}_{2}$ (normociapnia) until normal end tidal levels were attained ( $\pm 5 \mathrm{kPa}$ ). During all inductions and baselines subjects performed cognitive tasks, including a working memory and a long term memory task, and a task measuring visual perceptual sensitivity. No effect of inductions were seen on memory functions. Hence, hyperventilation was not shown to be a better model as compared to mild hypoxia. Caffeine appeared to impair working and long term memory functions, probably through enhancing arousal to a level which was detrimental to task performance. In addition, although hypoxia decreased the visual perceptual sensitivity on the signal detection task, no effect was seen of caffeine on this task.

It was concluded that caffeine was not able to reverse the cognitive dysfunction induced by hypoxia. Furthermore, different models of cognitive dysfunction, such as scopolamine and hypoxia, can be used to observe the profile and mechanism of action of potential new oognition enhancers.
\end{abstract}

\section{Introduction}

Caffeine is considered to act as a central stimulant and to have effects on cognitive functioning through enhancing alertness and vigilance (Bättig, Buzzi, Martin, Feierabend, 1984; Lieberman, Wurtman, Emde, Roberts, \& et al, 1987; Loke, 1988). However, direct effects of caffeine in attenuating memory dysfunctions in the scopolamine model have recently been found (Riedel, Hogervorst, Leboux, Verhey, Praag, \& Jolles, 1995). In this study, caffeine also positively affected the scopolamine-induced decreased visual perceptual sensitivity. We argued that caffeine may have acted as a cognition enhancer and that future potential cognition enhancers should have effects which are at least equal to or superior to the effects of caffeine.

The need for cognition enhancers to treat age-related cognitive impairments is recognized, but is as yet not reflected by the number of registered medicinal drugs. Valid, reliable, and safe models 
of age-related cognitive deficiency are needed to tert the potential effect of cognition enhancers in homogeneous groups of young volunteers. Scopolamine is considered to be such a model. It is judged to be adequate for the studly of age-related cognitive deficiencies and Alzheimer"s dementia, since it model the cholinergic deficiency thought to be implicated in these processes (Bartus, Dean III, Beer, \& Lippa, 1982). Caffeine may have reversed the cholinergic antagonism in the scopolamine model by acting as an adenosine antagonist and thus indirectly increasing cholinergic function (Biaggioni, Paul, Pucket, \& Arzubiaga, 1991). However, scopolamine is also known to reduce the cerebral blood flow and to decrease cerebral metabolism (Gitelman \& Prohovnik, 1992; Honer, Probonnik, Smith, \& Lucas, 1988). Since caffeine has been noted to increase cerebral metabolism (Neblig, Daval, \& Debry, 1992), it may have affected memory functions, alternatively, through these mechanisms. Testing caffeine in a model of decreased cerebral metabolism may further illuminate these matters. The present study was devised for that purpose.

Several models have been proposed in which the oxygen avalabillity to the brain is reduced experimentally. The experimental induction of hypoxia is such a model and has been used to test the effect of cognition enhancers (Kraaier, 1988; Saletu \& Grunberger, 1984; Schlaffer, Hormann, \& Anold, 1981). In mild hypoxia $\left(11.6 \% \mathrm{O}_{2}, 3000-4000 \mathrm{~m}\right.$ altitude) negative effects on wisual sensitivity and reaction time tasks have been reported (Noble, Jones, \& Davis, 1993; Fowler, Taylor, \& Porlier, 1987, Cavaletti \& Tredici, 1993). However, the effects of mild hypoxia on mentory functions are disputed (Denison, Ledwith, \& Poulton, 1966; Noble, Jones, \& Davis, 1993; Fowler, Paul, Porlier, Elcombe, \& Taylor, 1985). According to Kraaier, van Huffelen \& Wieneke (1988; 1989) and Rother, Kessler, Funke, Rother, \& Windisch (1994), standardized hyperventilation (HV) is a more adequate and safer model to induce cognitive dysfunction due to a concomitant decrease in the cerebral blood flow. The authors stated that detrimental effects of hyperventilation on short term or working memory were seen to accur. However, no control procedures were used in these studies. We report elsewhere, however, that control procedures (wearing a mask and/or with additional $\mathrm{CO}_{2}$ ) are essential when evaluating the effect of hyperventilation and/or hypoxia on test performance (Hogervorst et al, submitted).

In the present study the HV and hypoxia models of cognitive dysfunction were further investigated in terms of the possible effects of caffeine. The effect of the inductions on working memory capacity. long term memory, and visual sensitiwity were measured. Hypotheses were that caffeine would anhance memory functions and reverse the decreased visual sensitivity in both the hypoxid and hyperventilation model. The hypotheses were based upon earlier findings with the scopolamine model (Riedel et al, 1995).

\section{Method}

\section{Subjects}

20 male volunteers were recruited through advertisement. Subjects were aged between 23 and 30 years (nean age $24 \mathrm{yrs}$ ). All subjects ingested caffeine on a habitual basis (on average 3 cups/day). Subjects were free of cardiac, hepatic, renal, pulmonary, neurological, gastrointestinal, hematological, and psychiatric disease as determined by extensive medical evaluation including history, physical examination, and routine ECG. The study was approved by the Medical Ethical Committee of the University and the Academic Hospital Maastricht. Subjects gave their informed consent after reading "Information for volunteers" before the start of the study. Payment to the volunteers for their time $(12.5 \mathrm{~h})$ and inconvenience was equivalent to 75 dollars. 


\section{Design}

This study had a mixed design. Subjects were at random assigned to start with either one of the two inductions (hypoxia or hyperwentilation, each with their own control procedure: normoxia or normocapnia) and after a one week interval crossed over to the other induction. The orders of normocapnia and hypocapnia and normoxia and hypoxia were counterbalanced according to a 4 $x 4$ Latin square. The baseline measurement was always done in the middle. Subjects were kept naive of the actual induction throughout the procedure (single blind). Caffeine was tested with a parallel groups design. Subjects were matched for habitual caffeine use (on average 3.0 cups/day SD 1.5) and randomlly assigned to either the $230 \mathrm{mg}$ caffeine or the placebo treatment in a double blind fashion.

\section{Procedure}

Priar to the experiment there was a training session in which the hyperventilation procedure, the breathing of hypoxic air through the mask (as a challenge procedure), and the cognitive tasks were trained. Prior to the testing days, subjects were asked to attempt to sleep normally and to refrain from any caffeinated drinks (the morning before testing) and medication. including 'social' drugs (e.g. marijuana) and alcohol (the latter within 24 hours prior to the experiment).

\section{Time schedule:}

$\begin{array}{llllll}0 & & & & \text { After } \\ 3 \text { cups } & 1 & 3 & 3.5 & 4 & \text { hours } \\ \text { coffee } & \text { Induction } & \text { Baseline } & 1.5 \text { cups } & \begin{array}{l}\text { Induction } \\ \text { coffee }\end{array} & \text { Tests }\end{array}$

The procedure on testing days was as follows. First, subjects gave a saliva sample and then received caffeine or placebo. After a short training, subjects started learning a list of 15 words in five consecutive presentation trials (Visual Verbal learning Test, VVLT, see below for description). Then, the mask was put on and the induction or its control procedure began. During the first 10 minutes no tests were done in order to avoid the influence of early primarily stress-related responses (Hout van den, de Jong, Zandbergen, \& Merckelbach, 1990). During the $30 \mathrm{~min}$ that followed, subjects performed a dual attention task measuring working memory functions and the visual sensitivity task. After these tasks, the mask was put off and subjects were tested for delayed recall of the words learned prior to the induction (VVLT delayed recall). Another saliva sample was taken. One hour later a baseline measurement without a mask wass done. Subjects did the cognitive tests in the same order but did not breath through the mask. Afterwards, subjects received a maintenance dose of caffeine (115 $\mathrm{mg})$ or placebo. Three hours after the first procedure the control procedure followed (time schedule). Then subjects gave their final saliwa sample and were asked if they knew what condition they had received to ensure the single blind procedure. Subjects were not able to differentiate between control or actual induction.

\section{Apparatus and inductions}

An infrared gas analyzer (Datex Multicap(B) Multigas Monitor; CNO-103) was used for capnographic control with continuous sampling of gas during all inductions. The gas was sampled at a flow rate of $500 \mathrm{ml} / \mathrm{min}$ through a side tube of $190 \mathrm{~cm}$ length connected 10 a fully sealed oral nasal mask (Eros; F5341). Samples of the end tidal $\mathrm{CO}_{2}$ levels, and $\mathrm{SaO}_{2}$ were recorded every 2 min on a sheet by an experimenter. $\mathrm{SaO}_{2}$ was measured continuously with a fingeroximeter (Datascope(B)) connected to an oximeter (Datascope(B) Passport Monitor). Respiration rate $(R R)$ was recorded every min by counting the number of inhalations by two 
independent tegters. Average $\mathrm{CO}_{2}, \mathrm{SAO}_{2}$, and $\mathrm{RR}$ were calculated over the actual testing period, which took $30 \mathrm{~min}$

\section{Inductionge}

Hypoxia was induced by inhalation of a gas mixture contained in a high pressure bottle filled. with $13.6 \% \mathrm{Q}_{2}$ and ballanced with nitrogen. This gas mixture is the equilwalent of air at an altitude of $3500 \mathrm{~m}$ or $11650 \mathrm{ft}\left(\mathrm{SaO}_{2}\right.$ on average reached: $88.5 \%$ (SD:2.63). A gas mixture containing normal air ( $21 \% \mathrm{O}_{2}$, werage $\mathrm{SaO}_{2}$ reached: $98.24 \%$ ( $\left.\mathrm{SD} .96\right)$ was breathed through the mask as a control. Valves, operated manually, reduced the pressure from the cylinders to $5.52 \times 105 \mathrm{~N} \mathrm{x} \mathrm{m}^{2}$ (or ambient lewel). The breathing mixture was connected to the orall-nasal mask via $190 \mathrm{~cm}$ of a flexible, corrugated tubing with an inside diameter of $0.7 \mathrm{~cm}$. For the byperventilation and normocapnia inductions, subjects received normal air through the open mask. Hyperventilation was induced by inereasing the breathing frequency of the subject up to $\pm 60 / \mathrm{min}$, until an end-tidal $\mathrm{CO}_{2}$ of on average $2.2 \mathrm{kPa}$ (SD:.21) was reached. Breathing instructions were given by the experimenter "Breath in and out as deep and fast as you can". In the normocapnic induction, the mask was connected to a tank containing $100 \% \mathrm{CO}_{2}$. The pressure of $\mathrm{pCO}_{2}$ into the tubing connected to the open mask was low: 1,5 bart and increased $\mathrm{pCO}_{2}$ up to $5 \%$ (as compared to the percentage in normal air: $0.2-0.3 \%$ ) to compensate for the excess aspired $\mathrm{CO}_{2}$. As such, a lowered $\mathrm{pCO}_{2}$ in arterial blood, as is seen in hyperventilation, was not provoked by this method. In control subjects arterial $\mathrm{CO}_{2}$ levels thus remained at normal levels (end-tidal $\mathrm{CO}_{2}$ of on average $4.91 \mathrm{kPa}(\mathrm{SD}: 47$ ). Recovery took place by instructing subjects to breath normally through the nose.

\section{Caffeine}

Caffeine is readily absorbed after oral administration. Its maximal plasma concentration is achieved within 1 hour. Caffeine, in adults, has a half-life in plasma of 3 to 7 hours. $230 \mathrm{mg}$ of caffeine (in 3 cups) was administered one hour before the inductions. Identically appearing decaffeinated coffee was used as a placebo. One hour before the second induction, subjects received a maintenance dosage of $115 \mathrm{mg}$ (in 2 cups) caffeine or placebo.

\section{Saliva Caffeine analysis}

Saliva samples were taken before each experimental session and after (at an average of $75 \mathrm{~min}$ after the last cup of coffee). Samples were stored at $-22^{\circ} \mathrm{C}$. Saliva caffeine concentration was analyzed using high-performance liquid chromatography method and made possible by a grant from the Institute for Scientific Information on Coffee, Paris.

\section{Cognitive tests}

Working memory capacity task. Subjects were briefly shown a set of three letters and told to memorize them. This is called the "memory set" (Stemberg, 1969). Subjects then saw an series of 48 letters displayed for $1000 \mathrm{msec}$ on a computer screen followed by another $2000 \mathrm{msec}$ before the next letter appeared. Half of the presented letters were part of the memory set and had to be recognized as targets. The subjects" task was to decide and respond as rapidly as possible by pressing either "YES" or 'NO' to indicate whether or not each successive letter was one of those contained in the memory set. The median of their response time (RT) is taken as a dependent parameter (Brand \& Jolles, 1988). Subjects were again shown three letters but now had to count the number of times each target was presented. At the end of the task subjects were asked to report the three individual target frequencies. In the last subtask, subjects had to respond to each letter by pressing the 'Yes' or 'No' button and count simultaneously the number of times that each target was presented. In this fashion, Perceiver Operating Characteristics (POC) is devised, by calculating the performance of the two variables (counting and RT) of subtask 5 as the deviance (in percentages) of the two scores of subtask 3 (RT) and 4 (counting), which were transformed 
into maximum performance $(100 \%)$ scores. All scores are then plotted in a curve. The surface above the curve is calculated, which is called the Diwided Attention Cost (DAC). The surface of the DAC represents the additionall slowing when two tasks are done simultaneously (Ponds; Brouwer, \& Van Wolffelaar, 1988; Salthouse Davenport Rogan \& Prill, 1984). In the current task. the DAC can be seen as a measure of working memory capacity, since simulatenous storage and processing functions of working memory are tested (Baddeley, 1986).

Long term memory task (Visual Verbal Leaming Test with Distraction. WVLT) One of six parallel lists of 15 monosyllabic meaningful nouns matched across word frequency, were presented on a computer screen with a rate of 2 seconds per word. The word was presented for $1 \mathrm{sec}$ and then the screen remained blank before another word was displayed (Brand \& Jolles, 1985). Simultaneously, 15 similar but different distractor nouns were presented auditorily. Each measurement, a different visual word list with a different auditory list was presented. List order was completely order balanced over inductions to avoid learning effects or list type interference. Subjects were asked to verbally recall as many of the visually presented words as soon as the presentation has stopped and not to interfere these with the (distracting) auditory words. This first immediate recall trial was followed by four more trials in which the same words were repeated in the same order. When the fifth trial was completed the subjects proceeded performing subsequent activities and after 30 minutes were asked to name as many of the previously learned words that were presented visually without prompting (delayed recall). Signal Detection Spatial Perception Task (Visual Sensitivity). In the visual search task, stimuli are presented in a pseudo-random order on a computer video monitor $(640 \times 480$ pixels resolution). Twenty stimuli are randomly assigned to a $10 \times 6$ grid, all stimuli being $2.5 \mathrm{~cm}$ apart. Stimuli are small squares of $3 \times 3 \mathrm{~mm}$; targets are defined as four stimuli forming a square of $2.5 \times 2.5 \mathrm{~cm}$. Every $1000 \mathrm{msec}$, three stimuli are moved to a different location in the matrix. Subjects are seated $60 \mathrm{~cm}$ from the monitor and have to respond to targets with their dominant hand by pressing a button as quickly as possible. At the center axis the visual angle horizontally is $11.80^{0}$; the visual angle vertically is 7.10. Thus subjects have to move their eyes to be able to detect all targets. Subjects have to respond to targets within $2000 \mathrm{msec}$, after that targets disappear. The total number of stimuli presented is 400 . With 56 targets ( 7 targets $\times 8$ blocks), this gives an overall signal probability of 0.』4. The task lasts $6.4 \mathrm{~min}$. Targets are never consecutive stimuli. The same number of stimuli are presented in each quadrant of the inspection area. Before the actual task is performed there is a practice session consisting of 15 stimuli with 3 targets in which subjects obtain feedback on task performance (Klein, Ponds, Houx, \& Jolles, submitted). The median reaction time is registered and the perceptual sensitivity is calculated. According to the theory of signal detection (Pollack \& Norman, 1964), the proportion of correctly recognized (cr) along with the proportion of fallse recognized (fr) constitute the non-parametric sensitivity measure: $A^{\prime}=1-1 / 4[\mathrm{fr} / \mathrm{cr}+$ $[1-\mathrm{cr}] /[1-\mathrm{fr}]]$. $\mathrm{A}^{\prime}$ is in fact the proportion of correctly recognized targets, corrected for the subject's response tendency. Because the distribution of $A^{\prime}$ is skewed due to a ceiling effect, $A^{\prime}$ is arcsin transformed before entering statistical analysis.

\section{Statistical Analysis:}

Dependent variables were analyzed in two separate (for hypoxia and hyperventilation procedures) repeated measures multivariate and univariate (MANOVA using SPSS 4.0 on Macintosh) analysis of variance, according to a 3 (induction) $\times 2$ (caffeine) factorial modell to test the effects of the inductions and of caffeine. The first within factor (induction) had 3 llewels: baseline (of the hypoxia procedure: $\mathrm{BO}$ or of the hyperventilation procedure: $\mathrm{BV})(1)$ control procedure (normoxia:NO or normocapnia:NV) (2) and hypoxia (HO) or byperventilation (HV) (3). The second between factor was caffeine, which had 2 levels caffeine (1) and placiebo (2). If overall 
interaction effectus were significant, univariate contrasts ithe effect of wearing a mask ( $\mathrm{NO}$, and overbreathing: NV) rellative to baseline (BO or $\mathrm{BV}$ ) and control procedures (NO or NV) relative to hypoxia or hyperventilation (HO or HW)] were regarded to investigate the nature of the caffeine $*$ induction interaction. Probabilities of $5 \%$ or less were considered significant (twotailled).

\section{Resullts}

\section{Silliwa caffeine levells}

No subjects had pretreatment caffeine saliva levels over $2.0 \mathrm{mg} / \mathrm{L}$, which suggested that all had adhered to the abstinence instructions. There was a significant overall effect of caffeine $[\mathrm{F}(1,18=105.39, \mathrm{p}<.0001]$, no significant main effect of testing days $[\mathrm{F}(1,18)=1.93, \mathrm{p}=\mathrm{ns}]$ or a significant interaction effect between testing days and caffeine $[F(1,18)=20, p=n s]$. However, as expected, there a significant main effect of test sessions $[F(2,17)=106,1 \mathbb{1}, p<.000]$ and al significant interaction between test sessions and caffeime $[F(2,17)=110.98, p<0001]$. Average pretreatment saliwa concentrations before the inductions were $.38 \mathrm{mg} / \mathrm{L}(\mathrm{SD}=.41)$, which were not to be different between conditions. [CAF:M=.49 (SD.58), PLAC:M=.27 (SD.24), $F(1,18)=.76$, $p=n s]$ or between the two baselines $[F(1,18)=.61$, $p=n s]$.

After caffeine had been ingested, for the caffeine group, no significant difference was seen in caffeine saliva levels [on average $=3.18(\mathrm{SD}=.72)]$ between the testing days $[\mathrm{F}(1,8)=1.65, \mathrm{p}=\mathrm{ns} \rrbracket$ or between the two test sessions $[\mathrm{F}(1,8)=2.10, \mathrm{p}=\mathrm{ns}]$. Similarly, for the placebo group, no significant difference was seen in caffeine saliva levels [on average $=30(\mathrm{SD}=.19)$ ] between test days $[F(1,10)=1.39, p=n s]$ or between test sessions $[F(1,10)=28, p=n s]$.

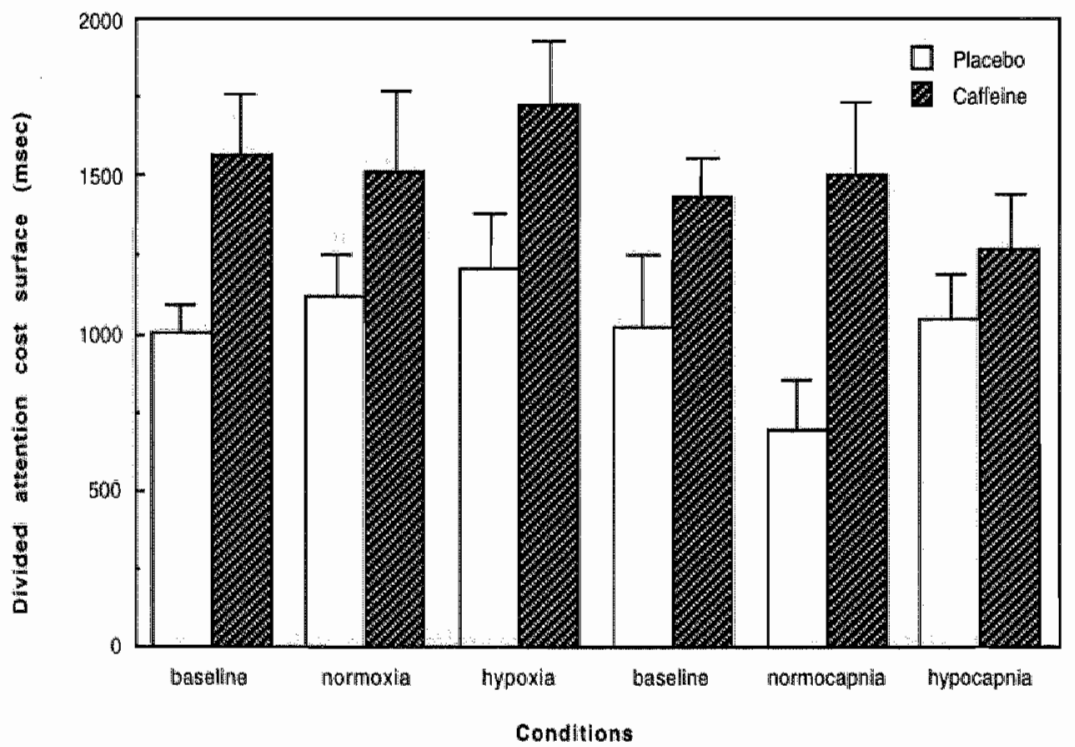

Fig 1. Mean surface (SE) of the divided attention cost (msec) over unductions with caffeine or placebo 


\section{Working memory functions}

Maltivariate analyses showed that performance on the working memory capacity task as measured with the POC was not affected by the hypoxia inductions $[E(2,17)=4, p=n s]$. However, at trend main effect of caffeine was detected $[E(1,18)=3.82,1=07]$. No interaction effect between induction and caffeine was found (see fig 1). For hyperventilation inductions, no multivariate main effect was seen $[\mathrm{E}(2,17)=15$, $\mathrm{D}=\mathrm{ns}]$. However, a significant main effect of caffeine was found $[E(1,18)=6.1, p<05]$, while no interaction effect could be detected. In general, caffeine increased the surface of the $\mathrm{DAC}$, meaning that counting and responding at the same time was more difficult after caffeine in all conditions. The surface of the DAC is a measure of working memory capacity. Thus, overall; caffeine had a negative effect on working memory capacity.

\section{Long term memory}

No multivariate main effect of hypoxia inductions $[\underline{E}(2,17)=84, p=n s]$ or of caffeine $[\mathrm{F}(1,18)=2.5, \mathrm{p}=\mathrm{ns}]$ was detected on the number of words recalled after $30 \mathrm{~min}$ (delayed recall). However, an interaction effect between hypoxia inductions and caffeine was found $[\mathrm{E}(2,17)=3,79$, $\mathrm{p}<.05]$. Univariate analyses between baseline and control condition showed no main effect of inductions or of caffeine. However, there was a significant interaction effect between induction and caffeine, in that there was at detrimental effect of caffeine and wearing a mask on the subsequent retrieval of words $[\mathrm{BO}-\mathrm{NO}, \mathrm{E}(1,18)=6.42, \mathrm{p}<.05]$ (Fig. 2a). Univariate analyses showed that there was no difference between hypoxia and normoxia on the retrieval of words $[E(1,18)=.00, p=n s]$. A main effect of caffeine was apparent $[E(1,18)=4.68,2<.05]$, but an interaction effect could not be detected

Similarly, multivariate analyses revealed no significant main effect of the hyperventilation inductions $[\mathrm{E}(2,17)=1.74, \mathrm{p}=\mathrm{ns}]$ or of caffeine $[\mathrm{E}(1,18)=.37, \mathrm{p}=\mathrm{ns}]$ but did show an interaction effect between induction and caffeine $[\mathrm{E}(2,17)=3.57, \mathrm{p}=.05]$. Univariate analyses showed no difference between normocapnia and baseline or between normocapnia and hyperventilation. Hence, the interaction effect was explained by the combined effect of hyperventilation with caffeine intake as compared to the baseline induction $[\mathrm{E}(1,18)=7.4, \mathbb{2}=.01]$ (Fig. 2b)

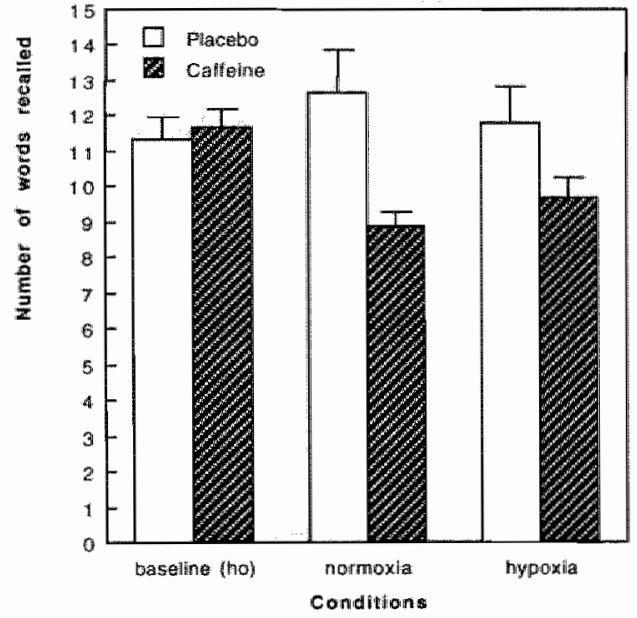

Fig 2a. Mean number of womts (SE) recalled ather a delay of $30 \mathrm{~min}$ after baseline, normoxia, and hypoxia with catteine or placebo

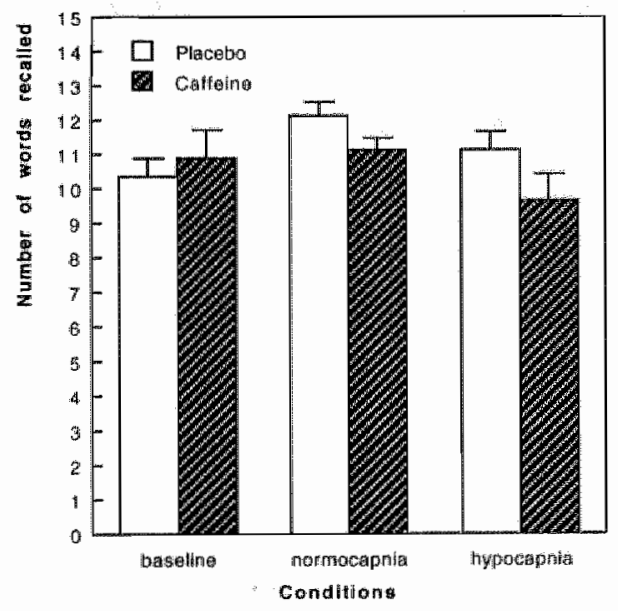

Fig 2b. Mean number of words (SE) recalled after a delay of 30 min alter baseline. normocapnia, and hypocapnia with calleine or placebo 


\section{Visual perceptual sensitivity}

There was a multivariate main effect of hypoxia inductions on the sensitivity measure of the SDT $[\mathrm{E}(2,17)=4.99, \mathrm{D}<05]$. There was no multivariate main $[\mathrm{F}(1,18)=2.42, \mathrm{D}=\mathrm{ns}]$ or interaction effect $[E(2,17)=49, \mathrm{p}=\mathrm{ns}]$ of caffeine. Uniwariate analyses between baseline and normoxia revealed no significint effects. Univariate analyses between hypoxia and normoxia showed that the effect of inductions on visual sensitivity was not attributed to a mask effect $[\mathrm{BO}=\mathrm{NO}]$ but was an actual effect of the hypoxic induction on the sensitivity $[\mathrm{NO}<\mathrm{HO}, \mathrm{E}(1,18)=7.21, \mathrm{p}<.05]$. No effects of caffeine were detected (see table 1). No multiwariate main effect of the hyperventilation inductions on the sensitivity measure was found $[\mathbb{E}(2,17)=1.36, p=n s]$. There was no multivariate main $[\mathrm{E}(1,18)=1.33, \mathrm{p}=\mathrm{ns}]$ or interaction $[\mathrm{E}(2,17)=60, \mathrm{~g}=\mathrm{ns}]$ effect of caffeine (see table 1 )

Table 1 : Outcome (mean and stdev) of performance on the visual sensitiwity parameter of the Signal Detection Task over inductions with caffeine $(n=10)$ or placebo $(n=10)$.

\begin{tabular}{|c|c|c|c|c|c|c|}
\hline \multirow[t]{2}{*}{ Induetions } & \multicolumn{2}{|l|}{ Baseline } & \multicolumn{2}{|c|}{ Control } & \multirow{2}{*}{\multicolumn{2}{|c|}{$\begin{array}{l}\text { Actiwe induction } \\
\text { Caff Pla }\end{array}$}} \\
\hline & Caf & Pla & Caf & Pla & & \\
\hline $\begin{array}{l}\text { Hypoxia (BO, } \mathrm{NO}, \mathrm{HO}) \\
\text { Hyperwentilation (BV, } \mathrm{NV}, \mathrm{HV})\end{array}$ & $\begin{array}{l}96(05) \\
93(05)\end{array}$ & $.91(08)$ & $.94(.07)$ & $.91(.06)$ & $91(05)$ & $.88(.07)$ \\
\hline & $93(05)$ & $.92(.07)$ & $.93(.08)$ & $.90(.08)$ & $.90(.10)$ & $.85(.12)$ \\
\hline
\end{tabular}

\section{Discussion}

This study evaluated the effects of caffeine $(230 \mathrm{mg})$ in two experimental models of cognitive dysfunction. Effects on working memory capacity, long term memory, and visual sensitivity were investigated. Since caffeine has been reported to positively affect cerebral metabolism (Nehlig, Daval, \& Debry, 1992), it was hypothesized that caffeine would attemate the effect of the inductions (hypoxia and hyperventilation) on cognitive performance. However, the results were contrary to expectations. First, hypoxia did have a negative effect on the visual perceptual sensitivity, but no effects of hypoxia or hypocapnic hyperventilation were seen on memory functions. Since at higher simulated altitudes $\left(> \pm 5000 \mathrm{~m}, \mathrm{SaO}_{2} 78-85 \%\right)$ hypoxia appears to impair memory functioning, similarly to scopolamine, (Cavaletti \& Tredici, 1993; Ernsting, 1978), the hypoxia induction may have been too mild to induce clearcut dysfunctions on working memory capacity or long term memory. As for hyperventilation no effect on memory or on wisual sensitivity were seen, which was similar to earlier findings (Hogervorst et al, submitted).

Second, caffeine appeared to have an overall negative effect on the working memory capacity task. In addition, a negative effect of caffeine on long term memory functions (delayed recall) was seen while wearing a mask and while hyperventillating. By contrast, wisual sensitivity was not affected by caffeine. These results are discrepant with the expectations that caffeine would improve cognitive performance under conditions of experimentally induced performance decrements. In an earlier study, clearcut beneficial effects of $250 \mathrm{mg}$ of caffeine were found in the scopolamine model of cognitive deficiency, where the drug attenuated the decrease in memory performance and visual sensitivity after scopolamine injection (Riedel et al, 1995). Several factors are relevant in explaining these seemingly discrepant results.

In the first place, caffeine is known to inhibit the actions of adenosine (Nehlig \& Debry, 1994). Adenosine has been found to have protective effects during brain ischemia (Higgins, Hosseinzadeh, MacGregor, Oglivy, \& Stone, 1994). Also, caffeine has been seen to increase the 
glucose metabolism in the brain, while decreasing the cerebral blood flow through its local vasoconstrictive actions (Nehlig, Daval, \& Debry, 1992). These effects of caffeine could thus be considered to be detrimental during brain ischemia. If caffeine would have had negative effects in hypoxia and hypocapnic hyperventilation only, these mechanisms could have been explanatory. However, caffeine was seen to have an owerall negative effect on working memory capacity and a negative effect on long term memory while wearing a mask as well.

Hence, alternative explanations need to be sought for the negative effects of caffeine on memory functions. There is an increasing number of studies which show that caffeine may exert dosedependent effects on cognitive functions. Loke (1988) reported in her literature review that the memory span has been seen to increase with low (e.g. $162 \mathrm{mg}$ ) but not with higher dosages of caffeine $(325 \mathrm{mg})$. In the present study, $230 \mathrm{mg}$ was used which is the equivalent of 3 cups of cofree consumed in $15 \mathrm{~min}$. Possibly, the negative effect of caffeine may be due to the relatively high dosage used, since similar dosages of caffeine (>225 mg) have also been shown to lead to impaired short term or working memory functions (Anderson, Revelle, \& Lynch, 1989; Foreman, Barraclough, Moore, Mehta, \& Madon, 1989; Hogervorst, Riedel, Schmitt, \& Jolles, submitted). The effects of different caffeine dosages on cagnitive functions may thus follow an inverted $U$ shaped curve (for a general review, see Watters, Martin, \& Schreter, 1997). Since an increase in arousal is known to occur after caffeine intake (Anderson, Revelle, \& Lynch, 1989; Anderson \& Revelle, 1994; Nehlig, Daval, \& Debry, 1992; Lorist, Snel, Kok, \& Mulder, 1994), the effect of caffeine on cognitive functions could be explained through the Yerkes-Dodson law (Yerkes \& Dodson, 1908) which predicts a negative quadratic relationship between arousal and performance ('inverted-U hypothesis'). A high level of arousal may reduce the capacity for a broader range of cue utilization, which is required by the simultaneous execution of two tasks (Easterbrook, 1959). With respect to long term memory, complex interactions occurred between wearing a mask (normoxia and hypoxia), hyperventilation, and caffeine on the number of words recalled after a delay of $30 \mathrm{~min}$. The optimum level of arousal is task-dependent (Yerkes-Dodson law, 1908), which may explain the difference in outcome between working and long term memory. Indeed, delayed recall has been shown to increase with 75 and $150 \mathrm{mg}$ of caffeine (Warburton, 1995), while higher dosages (> $200 \mathrm{mg}$ ) had no effect on long term memory (Hogervorst, Riedel, Schmitt, \& Jolles, submitted; Loke; 1988). The earlier study showed that wearing a mask and overbreathing increased the heart rate which is an indicator of increased arousability (Hogervorst et all, submitted). A plausible explanation would then be that the inductions caused an additional arousal which, combined with the increased arousall due to caffeine consumption, interfered with retrieval of information from long term memory. High levels of arousal and stress have been seen to reduce memory functions, through decreasing the clustering of information, search processes, and retrieval from semantic memory (Hasher \& Zacks, 1979). Automatic (e.g. trained and overlearned) task are less susceptible to disruption by high levels of arousal (Norman, 1979). Since the visual detection task was trained thoroughly, it may have been that the visual perceptual detection task demanded less cognitive capacity (e.g. in attention and effort) and hence was less susceptible to the increase in arousal. Alternatively, the relatively small number of subjects, combined with the large variation in performance, may not have been sufficient to show significant caffeine effects on the visual sensitivity.

In sum, in the present study, caffeine was seen to interact with non-specific experimental conditions, such as wearing a mask or overbreathing, which subsequently gave a stress-associated decrement in memory performance. Contrary to expectations, caffeine could not reverse the actual hypoxia effect on visual sensitivity, while it did earlier reverse the scopolamine induced deficiency. It was stated that valid, safe, and reliable models of age-related deficiencies are needed 
to evaluate the effect of cognition enhancers (Hogervorst et al, 1997). As for the validity of the models, the effect of both seopolamine (Brandeis, Naylor, Halliday, Callaway, Yano, 1992) and mild lypoxia (Hogervorst et al, 1997, Kidf \& Imail, 1993) have hypothesized to be exerted at the level of attention, in the inthibition of the optimal utilization of attentional resources. In addition, muld tyypoxia has been shown to act on complex psychomotor functions (Hogervorst et al, 1997). In this respect, both the hypoxia and scopolamine can be regarded to be separate, adequate models for age-felated cognitive irmparment, since, during aging, a reduction in cognitive capacity and the ability to perform effortful processes is seen to occur (Hasher \& Zacks, 1979). Although caffeine can be regarded as a cognition enhancer, in that it reverses cognitive deficiencies in some models, (scopolamine, benzodiazepines), it was not shown to be a valid golden standard drug for models which test cognitive impairment associated with a reduced brain oxygen availability. However, the scopolamine study (Riedel et al, 1995) showed that caffeine can be a valuable control drug in studies into the effect of cognition enhancers acting upon excitatory neurotransmitter functions, such as acetylcholine or monoamines. It is of relewance in this respect 10 consider the notion proposed by Coper and Kanowski (1983). These authors stated that a specific uniform mechanism of action; i.e. pathway, for a cognition enhancer often is not known. Therefore, we propose to use differential models to observe the profile of drug effects in these warious models. The present study, where caffeine has effects which are different from those obtained in other models, underlines this notion and suggests that the mild hypoxia model may be relevant in this respect, to be used in addition to the more ubiquitously used scopolamine model. 


\title{
Chapter 9
}

\section{Caffeine in Carbohydrate Electrolyte Solutions improves Cognitive Performance before and after Strenuous Physical Exercise}

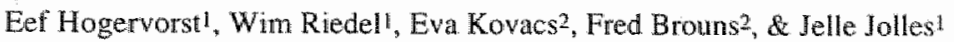 \\ I Brain \& Behaviour Insutute "European Graduate School for Netrosciences, Depurtment of Paychintry and \\ Neuropsychology \\ 2 Department of Human Biology
}

\begin{abstract}
The effects of three carbohydrate electrolyte solutions (CES) containing different amounts of caffeine on cognitive function and the combined effects of these drinks and exercise on cognitive functions were investigated in a double-blind, cross-over study.

On five separate occasions, fifteen endurance trained male athletes (23.3 years) received water placebo, CES placebo $(68.8 \mathrm{~g} / \mathrm{L})$, and three CES drinks containing low, medium and high dosages of caffeine $(150,225$, and $320 \mathrm{mg} / \mathrm{L})$. The drinks were consumed before an all-out 1 hour time trial on a bicycle ergometer $(8 \mathrm{ml} / \mathrm{kg})$ and during exercise $(6 \mathrm{~m} / \mathrm{kg})$. Psychomotor and cognitive tasks, long-term memory, perceptual interference, visual signal-detection, and choice reaction time, were carried out immediately before and immediately after exercise.

Before exercise, long-term memory was improved by CES plus low dose caffeine when compared to both placebos. Immediately after exercise, cognitive performance was improved by CES plus low- and medium-dose caffeine compared to both placebos. The cognition-enlancing effects of caffeine could not be explained by vigilance-enhancing effects before exercise or restotation of deteriorated psychomotor and cognitive performance due to fatigue after exercise.

These results comprise the first practical demonstration of the cognition enthancing effects of addition of low amounts of caffeine in CES before and after strenuous physical exercise.
\end{abstract}

\section{Introduction}

Caffeine has been shown to have beneficial effects on physical and mental performance. In several studies the endurance performance of trained athletes was prolonged after caffeine intake as compared to placebo. Caffeine intake was hypothesized to decrease physical fatigue (Costill et al. 1978; Essig et al. 1980; Ivy et al. 1979). Caffeine improves cognitive and psychomotor functions, especially during mental fatigue, which occurs, for instance, during sleep deprivation and monotonous cognitive tasks (Lorist et al. 1994b; Nehlig et al., 1992). These eflects of caffeine on cognitive functions hawe been explained by its pharmacological actions in the brain (Lorist el al., 1994a; Riedel et al., 1995).

Several studies have described a negative effect of strenuous physical exercise on the performance of cognitive tasks (Gutin, 1973; Hancock \& McNaughton, 1986; Isaacs \& Pohlman, 
1991; McMorits \& Keen, 1994; Salmela \& Ndoye, 1986). Thus, fatigue could have an important role in all typess of exercise in which concentration, reaction times, and technical/tactical skills have a major influence on both physical and mental performance, e.g. in most ball game sports or in ultra endurance events (Fleury et al., 1981; Hancock \& McNaughton, 1986; Isaacs \& Pothman, 1991). However, other studies failed to find a negative effect of strenuous exercise on cognitive functions (Adam et al. in press; Bard \& Fleury, 1978; Cote et al ., 1992; Fleury et al, 1981; Paas \& Adam, 1991; Tomporowski ef al., 1987). For instance, in a study with athletes, we found that cycling for one hour on a bicycle ergometer improved performance on cognitive tests (Hogervorst et al,, 1996). The enhanced activation and the subjects" expectancy that exercise would have a positive effect were thought to have been responsible for the enhanced cognitive performance.

In the present study, we inwestigated the effect of different doses of caffeine $(0-5 \mathrm{mg} / \mathrm{kg})$ in a carbohydrate electrolyte solution (CES), the effect of strenuous exercise (an all-out 1 hour time trial), and the combined effect of caffeine and exercise on several cognitive domains (memory and attentional functions) in a double-blind, cross-over study with trained athletes. Cognitive tests were administered after warming-up, just prior to the exercise time trial and immediately after exercise. We hypothesized that caffeine would enhance cognitive functions before and after exercise. Despite the vast amount of literature describing the physicall or the psychological effects of caffeine, a combined study has, to the best of our knowledge, never been carried out.

\section{Method}

\section{Subjects}

Fifteen healthy male competitive cyclists or triathletes, aged 23.3 years ( $S D=3.6)$, participated in the study. All subjects trained on a regular basis ( $\geq 2 \mathrm{~h} /$ day and $\geq 4$ times/week). Mean weight of the subjects was $72.6 \mathrm{~kg}(\mathrm{SD}=7.5)$ and their mean height. was $185 \mathrm{~cm}(\mathrm{SD}=8)$. None of the subjects had a known caffeine intolerance and all subjects regularly consumed caffeine (mean $148 \mathrm{mg} / \mathrm{day}$; $\mathrm{SD}=11.7$; range: $19-373 \mathrm{mg} / \mathrm{day})$. Subjects were aware of the risks involved in participation of the study and had signed an informed consent form. This study was approved by the local medical ethics committee at the University Hospital.

\section{Trearment}

The treatment consisted of drinking $(14 \mathrm{ml} / \mathrm{kg}$ body weight) water and four carbohydrate electrolyte solutions (CES) containing: carbohydrates $68.8 \mathrm{~g} / \mathrm{L}$, and carbohydrates $68.8 \mathrm{~g} / \mathrm{L}$. combined with caffeine $150 \mathrm{mg} / \mathrm{L}$, with caffeine $225 \mathrm{mg} / \mathrm{L}$ and with caffeine $320 \mathrm{mg} / \mathrm{L}$ (Table 1 ). Plasha levels of caffeine were determined at baseline, before and after cognitive testing, during the time trial and immediately thereafter (see figure 1). The four CES drinks further contained Vitamin C $100 \mathrm{mg} / \mathrm{L}$, Vit E $6 \mathrm{mg} / \mathrm{L}$, Na+ $690 \mathrm{mg} / \mathrm{L}$, Cl- $300 \mathrm{mg} / \mathrm{L}$, K+ $180 \mathrm{mg} / \mathrm{L}$, Mgt+ $10 \mathrm{mg} / \mathrm{L}$, $\mathrm{Ca}+10 \mathrm{mg} / \mathrm{L}$, phenylalanine $70 \mathrm{mg} / \mathrm{L}$, tyrosine $70 \mathrm{mg} / \mathrm{L}$, taurine $70 \mathrm{mg} / \mathrm{L}$, myoinositol $51 \mathrm{mg} / \mathrm{L}$ and choline $100 \mathrm{mg} / \mathrm{L}$.

The experiment was conducted according to an order balanced within subjects repeated measures design. The five drinks were studied according to a double-blind, placebo-controlled, cross-over design: placebo water (Pla-W), placebo carbohydrates (Pla-CES), caffeine $150 \mathrm{mg} / \mathrm{L}$ (CES-150), caffeine $225 \mathrm{mg} / \mathrm{L}$ (CES-225) and caffeine $320 \mathrm{mg} / \mathrm{L}$ (CES-320) respectively. Treatment sequences were drawn at random. 


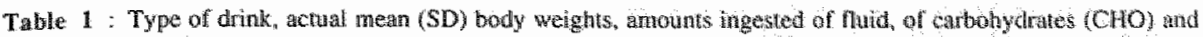
of caffeine, durirg warming up (before exercise) and the respective cumalative total anounts ingested.

\begin{tabular}{|c|c|c|c|c|c|c|c|}
\hline Drink & $\begin{array}{l}\text { Body Weight } \\
\text { (Kg) }\end{array}$ & $\begin{array}{l}\text { Fuid inuake } \\
\text { berore (mil) }\end{array}$ & $\begin{array}{l}\text { Total flutud } \\
\text { Intated (m) }\end{array}$ & $\begin{array}{l}\text { CHO intak } \\
\text { before (g) }\end{array}$ & $\begin{array}{l}\text { Total } \mathrm{CHO} \\
\text { intake }\end{array}$ & $\begin{array}{l}\text { Caflerino } \\
\text { intake } \\
\text { betore }(\mathrm{mg})\end{array}$ & $\begin{array}{l}\text { Tokal } \\
\text { caflese } \\
\text { inkme (nog) }\end{array}$ \\
\hline Pla-W & $73.3(7.6)$ & $586(61)$ & $1026(106)$ & 0 & 0 & 0 & 0 \\
\hline Pla-CES & $73.6(7.3)$ & $589(58)$ & $1030(102)$ & $40.5(4.0)$ & $70.9(7.0)$ & 0 & 0 \\
\hline CES- 150 & $73.3(7.3)$ & $586(59)$ & $1026(103)$ & $40.3(4.0)$ & $70.6(7.1)$ & $87.9(8.8)$ & $1537(15.4)$ \\
\hline CES-225 & $73.3(7.0)$ & $586(56)$ & $1026(99)$ & $40.3(3.9)$ & $706(68)$ & $131.9(12.7)$ & $230.9(22.2)$ \\
\hline $\operatorname{ces}-320$ & $73.4(7.3)$ & $587(58)$ & $1027(102)$ & $40.4(4.0)$ & $70.7(7.0)$ & $187.8(18.7)$ & $326.6(32.7)$ \\
\hline
\end{tabular}

\section{Procedure and apparatus}

Each subject came to the laboratory six times with a 1 -week interval between separate trials. In the first session, maximal workload was assessed and subjects were trained in the cognitive tests. Subjects were trained on all tests twice to prevent ongoing learning effects during the experimental sessions. Twenty-four hours before testing sessions began, subjects were not allowed any alcohol or caffeine containing drinks (coffee, tea, cola, chocolate, etc.).

A standardized breakfast was provided at the laboratory upon arrival. The test procedure, which started 1/2 hour after breakfast, consisted of a warming up (5 min 1.5 Watt / Kg Body Weight followed by $1.5 \mathrm{~min} 2.5 \mathrm{Watt} / \mathrm{Kg}$ Body Weight) session, during which the subjects were given one of the test drinks $(8 \mathrm{ml}$ fluid $/ \mathrm{kg}$ body weight).

After $20 \mathrm{~min}$, the first cognitive test session was carried out. This lasted 35 min. After a short warming up session ( 5 min $100 \mathrm{~W}$ ), the subjects took part in a simulated time trial during which they were given $3 \mathrm{ml}$ fluid/kg body weight after $1 / 3$ and again after $2 / 3$ of the time trial task. During this time trial, the subjects were asked to perform a certain amount of work (which required about 1 hour of cycling) as fast as possible.

The workload was based on the pretest maximal workload (Wmax) according to the formula:

$$
\text { Total amount of work }=0.75 * W \max * 3600
$$

The ergometer was set in the linear mode according to the formula: $W=L \cdot(R P M)^{2}$, in which RPM is the pedalling rate and $L$ is a linear factor. This factor was chosen in such a way that at pedalling rate of 90 RPM would result in $70 \%$ Wmax. In olluer words, the linear factor was dependent on a subject's Wmax. This would mean that $75 \%$ Wmax could be achired at about 100 RPM which appeared to be the preferential pedalling rate of most cyclists (Jeukendrup et al., 1996).

This validated exercise test can be classified as 'maximal' or "exhaustive' and subjects usually report maximal values on scales of perceived exertion (Salmela \& Ndoye, 1986). The subjects cycled on an magnetically braked ergometer (Lode Excalibur Sport ${ }^{\mathrm{R}}$, Lode BV, Groningen The Netherlands) and heart rate was measured continuously with a Sporttester (Polar, Finland (C). 


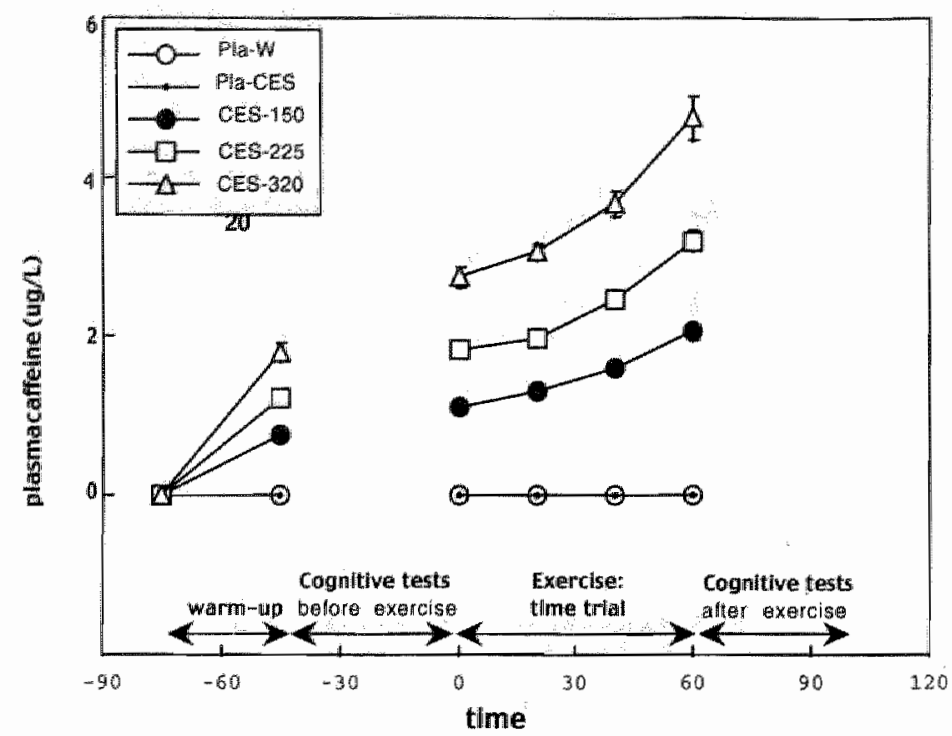

fligure 1: Mean: (t sem) plasma caffeine values as a function of test drinks and time (minutes) after ingeston of the drinks. Experimental events are also indlicated on the time axis.

Psychomotor and cognitive tasks

Subjects completed a test battery before and after exercise. The cognitive and psychomotor tasks are described below, and dependent variables are listed in table 2. The Visual Verbal Learning Test with Interference (VVLT) This test is an adapted version of the Rey Auditory Verbal Learning Test (Brand and Jolles, 1985; Lezak, 1995). The test consists of a list of 15 monosyllabic and concrete nouns in Dutch, which are presented in three trials on a computer screen. Simultaneously, 15 similar but different nouns are presented auditorily. The nouns occur very frequently and are acquired early in life. Items are presented in the same sequence at a rate of one every 2 seconds. Each trial ends with a free recall of the words. Thirty minutes after the third trial, the subject is requested to recall as many words as possible (free delayed recall). A yestho recognition test, consisting of the 15 former words and 15 new but similar words, is given after the delayed recall test. The reaction times are recorded. The variables used are: the number of correct words on delayed free recall as a measure of long-term memory and the median reaction time (RT) of correctly recognized target words as a measure of speed of retrieval from long-term memory. For each of the five treatment conditions, a different visual word list with a different auditory list was presented. Parallel lists were order balanced over treatment conditions. The word list was presented only before exercise. Delayed recall and recognition were assessed. before and after exercise. Because the distribution of recall is skewed due to a ceiling effect, recall data were arcsin transformed being used for statistical analysis. The Stroop Color-Word Test (SCWT: Lezak, 1995) is a perceptual interference task and consists of three cards. The test examines the speed at which color names are read (SCWT card I) and the speed at which colored patches are named (SCWT card II). The SCWT card III involves color names again, but the printing ink is different from the color name. The variables are: response times for cards I, II and III. Interference is expressed by the percentage of extra time needed for card III, trelative to card 
II. Before the start of each test the subject was allowed to practice one line. Sigmal Detection Task (SDT). In this visual search task, stimuli are presented in a pseudo-random order on a computer video monitor ( $640 \times 480$ pixels resolution). Twenty stimuli are randomly assigned to a $10 \times 6$ grid, all stimuli being $2.5 \mathrm{~cm}$ apart. Stimuli are small squares of $3 \times 3 \mathrm{~mm}$; targets are detined as four stimuli forming a square of $2.5 \times 2.5 \mathrm{~cm}$. Every $1000 \mathrm{msec}$ three stimuli are moved to a different location in the matrix. Subjects are seated $60 \mathrm{~cm}$ from the monitor and have to respond to targets with their dominant hand by pressing a button as quickly as possible. At the center axis the visual angle horizontally is 11.80 ; the visual angle vertically is 7.10 . Thus subjects have to move their eyes to be able to detect all targets. Subjects have to respond to targets within 2000 msec, after which the targets disappear. The total number of stimuli presented is 400 . With 56 targets, this gives an overall signal probability of 0.14 . The task lasts $60^{\circ} 40^{\prime \prime}$. Targets are never consecutive stimuli. Seven targets are presented every $50 \mathrm{sec}$ and the same number of stimuli are presented in each quadrant of the inspection area. Before each actual lask is performed there is a practice session consisting of one block in which subjects obtain feedback on task performance (Klein et al, 1997). The median reaction time is registered and the perceptual sensitivity $A^{*}$ is calculated. According to the theory of signal detection (Pollack and Nomman, 1964), the proportion of correctly recognized targets $(\mathrm{cr})$ and the proportion of falsely recognized targets(fr) constitute the non-parametric sensitivity measure: $A^{\prime}=1-1 / 4[\mathrm{fr} / \mathrm{cr}+[1-\mathrm{cr}] /[1-\mathrm{fr}]]$. $A^{\prime}$ is in fact the proportion of correctly recognized words, corrected for the subject's response tendency. Because the distribution of $A^{\prime}$ is skewed due to a ceiling effect. $A^{\prime}$ was aresin transformed before being used for statistical analysis. The Motor Choice Reacrion Time test (MCRT: Houx \& Jolles, 1993). Speed of information processing is assessed by measuring RT's as a function of task complexity. In the simple condition, basic psychomotor speed is assessed. The subject is requested to hold down, with the index finger of the dominant hand, a red home button. When a single target button lights up, the subject has to press this button as fast as possible with the same finger. In the choice condition a decision component is introduced. The subject has to press one of three possible buttons, positioned on a $900^{\circ}$ degree arc $5 \mathrm{~cm}$ above the central red button. These buttons light up in a random order. In the incompatible condition a response competition component is introduced. The subject has to press the button adjacent to the button that lights up. The home button has to be held down immediately after the target button was pressed until the next button lights up. The inter-stimulus interval of 500 or $1500 \mathrm{msec}$ is randomly varied. Each subtask consists of 40 stimuli, the first 10 of which are practice trials. The measures used for analysis are the median $\mathrm{RT}^{\mathrm{x}} \mathrm{s}$ of the simple, the choice, and the incompatible condition.

Table 2: Tests, dependent variables and functions measured in the present study

\begin{tabular}{|c|c|c|}
\hline Test & Dependent variables & Function measured \\
\hline VVLST & $\begin{array}{l}\text { Hof words }(0-15) \\
\text { RT (ansec) }\end{array}$ & $\begin{array}{l}\text { Storagge in long-term menory } \\
\text { Speed of retrieval from long-tern memory }\end{array}$ \\
\hline SCWT & Time to read card III. II (sec) & Perceptual Interference \\
\hline SHT & $\begin{array}{l}\text { RT (msec) } \\
\text { A. }^{\prime}(0.100)\end{array}$ & $\begin{array}{l}\text { Speed of wisuall signal detection } \\
\text { Efficiency of wisual signal detection }\end{array}$ \\
\hline MCRT & $\begin{array}{l}\text { Simple RT (mses) } \\
\text { Choice RT (msec) } \\
\text { S-R incompatible Choice RT (msec) }\end{array}$ & $\begin{array}{l}\text { Psychomotor spedd } \\
\text { Decision speed } \\
\text { Response Preparation Speed }\end{array}$ \\
\hline
\end{tabular}




\section{Statistices}

Dependent variables were analyzed with separate repeated measures analyses of variance (using the MANOVA module in SPSS 4.0 on Macintosh), according to a 5 factor model to test the effect of the test drink before and after exercise. The effect of exercise was evaluated by using paired t-tests comparing Pla-W before and after exercise. Separate paired t-tests were performed before and after exercise to assess caffeine-placebo differences (Tablle 3). These comparisons were made regardless of the outcome of the corresponding overall F-test. This is a legitimate procedure if the comparisons are built into the design or suggested by the theoretical basis of the experiment (Winer, 1971). However, it should be noted that whereas MANOVA is a conservative test, t-tests applied for a 'a priori' or 'planned' caffeine-placebo comparisons are not. Occasionally, one might expect a particulat comparison to show statistical significance on the basis of chance alone (i.e. as a type I error). Thus, care should be taken when MANOVA failed to show a significant overall treatment effect but t-tests indicated the occurrence of a significant caffeine-placebo difference. Hence, correction for multiple caffeine-placebo difference testing was accomplished by using the 'sequential Bonferroni' procedure for adjusting the alphaprobability criterion (Overall \& Rhoades, 1987).

\section{Results}

The data of one subject were omitted from analyses because caffeine was detected in the plasma during the CES trial. Therefore all analyses pertain to data for 14 subjects, unless otherwise indicated.

During the sessions, subjects were unaware of the content of the drink and were asked to guess the sequence of the five treatment conditions after each test session. Subjects were also asked the reasons for their choice (cycling performance, frequency of urination, subjective feeling, e.g. jittery, or increased concentration). The percentage of correct guesses was ( $\%$ of guesses based on taste is given between parentheses): CES-150: 22\% (13\%), CES-225: 38\% (19\%), CES-320: $41 \%$ (38\%), Pla-W: $50 \%$ (28\%) and Pla-CES: $63 \%(31 \%)$. Means and SD of dependent variables and the results of the t-tests before and after exercise are displayed in table 3. Drug effects expressed as caffeine-placebo difference scores are displayed in figure 2.

Before exercise, the test drink consumed had a significant multivatiate main effect on the delayed recall performance of the subjects on the VVLT $\left(\mathrm{F}_{4,10}=3.33, \mathrm{p}=.05\right)$. Tutests revealed that significantly more words were recalled after CES-150 than after both placebos.

After exercise the test drink did not have an overall effect $\left(\mathrm{F}_{4,10}=1.29\right.$, ns). Although the test drink did not affect delayed recognition $\mathrm{RT}$ before exercise $\left(\mathrm{F}_{4.9}{ }^{*}=2.10\right.$, ns), t-tests revealed that RT was significantly faster after CES-150 than after Pla-W. After exercise, there was a significant main effect of test drink on recognition $\mathbb{R T}\left(\mathrm{E}_{4,10}=3.52, \mathrm{p}<.05\right)$ which was significantly faster after both CES-150 and CES-225** than after Pla-CES.

There was no main effect of test drink on perceptual interference before exercise $\left(F_{4,10}=1.38\right.$, ns), but there was after exercise $\left(\mathrm{F}_{4,10}=3.88, \mathrm{p}<.05\right)$. Perceptual interference performance was significantly faster after CES-150, CES-225, and CES-320 than after Pla-CES, and after exercise than before exercise.

* Due to compruter failure, data of one subject were lost

* See discussion tor an explanation of this effect 
There was no main effect of test drink on visual signal-detection efficiency before exercise $\left(\mathrm{F}_{4,10}=1.23\right.$, ns) but there was after exercise $\left(\mathrm{F}_{4,10}=3,88, p<.05\right)$. Signal-detection efficiency was significantly better after CES-225 than after both Pla-W and Pla-CES and also wfter CES-150 than after Pla-CES.

There was no main effect of test drink on visual signal-detection RT before exercise $\left(F_{4,10}=1.70\right.$, ns), although, signall-detection RT was significantly faster after CES-225 than after Pla-W. After exercise, there was a significant main effect of test drink on signal-detection RT $\left(\mathbf{F}_{4,10}=5.35\right.$. p<.05) with signal-detection RT being significantly better after CES-225 than after Pla-CES.

There were no main effects of test drink on simple RT, either before exercise $\left(F_{4,10}=0.44\right.$, ns) or after exercise $\left(\mathrm{F}_{4,10}=0.43\right.$, ns). However, after Pla-W simple RT was significantly faster after exercise than before exercise.

There were no main effects of test drink on Choice $R T$, either before exercise $\left(F_{4,0}=1.64, n s\right.$ ) or after exercise $\left(\mathrm{F}_{4,10}=2.96, \mathrm{~ns}\right)$. However, after exercise choice $\mathrm{RT}$ was significantly faster after CES-150 than after Pla-CES.

There were no main effects of test drink on $S-R$ incompatible choice RT, either before exercise $\left(\mathbb{F}_{4,10}=1.36\right.$, ns) or after exercise $\left(F_{4,10}=1.94, n s\right)$. However, after exercise $S-R$ incompatible choice RT was significantly faster after CES-150 and after CES-225 than after Pla-CES. After Pla-W, S-R incompatible choice RT was also significantly faster after exercise than before exercise.
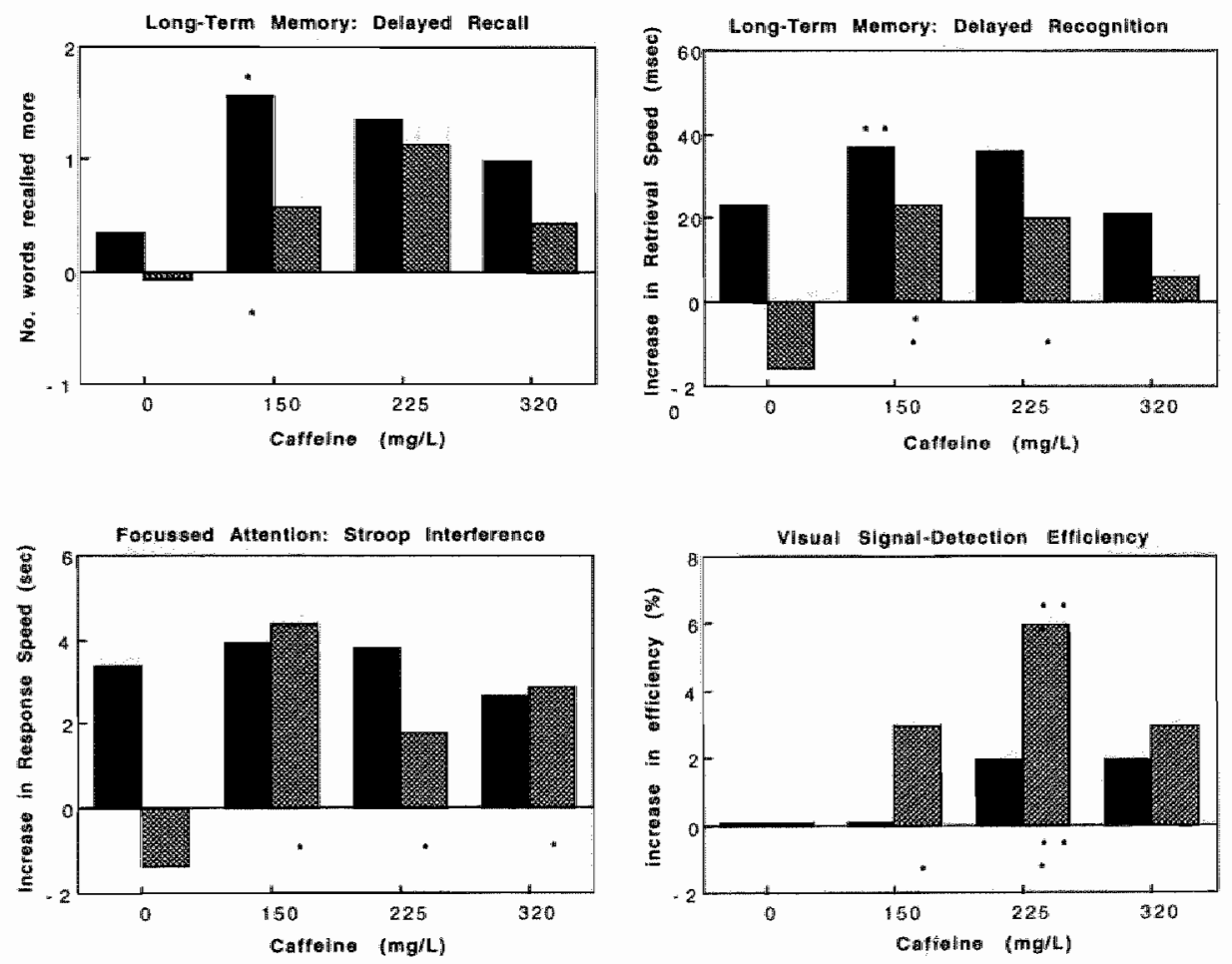

Figure 2 : Cognitive effects of caffeine-containing carbohydrate electrolyte solutions (0 mg corresponds with Pla-CES) before exercise (black bars) and after exercise (grey bars), expressed as demiations from placebo (Plan Wh. Henght of bars correspond with degree of improwement. : pe.05; * p p 01; ***; p<.005: Asterisks above bars refer to comparisons with PIaw $W$ and asterisks below bars refer to comparisons with Pla-CES 
108 I PARTI IT: CAFFEINE AND COCOMTION






\section{Discuission}

Our results confirmed the hypothesis that CES drinks containing caffeine have a positive effect on cognitive functions both before and after exercise: However, before exercise, the drink containing the lowest concentration of caffeine specifically improved long-term memory performance more than after both placebos, whereas after exercise, different concentrations of caffeine improved cognitive performance in general. Exercise itself also improved the speed of performance of certain tasks.

The effects of caffeine before exercise are comparable to those reported by other investigators who studied the cognitive effects of caffeine, whereas the effects of caffeine on cognitive performance after exercise are a new finding. For this reason we will discuss the effects of caffeine on cognitive performance before and after exercise separately.

It is possible that the order in which test drinks were given could have influenced the results. One way to quantify the order in which a treatment is given, is to calculate the arithmetic mean order. In the case of 5 treatments, these should ideally all be equal to, or approximate, 3 . The actual mean orders were: Pla-W: 3.07, Pla-CES: 2.79, CES-150: 3.14, CES-225: 3.36 and CES-320: 2.64. An average treatment order below 3 indicates that this particular treatment occurred slightly more often in the first two sessions than in the last two sessions whereas an average treatment order above 3 indicates that this particular treatment occurred slightly more often in the last two sessions. Assuming that most performance measures tend to improve over sessions, comparisons between treatments could result in a favourable outcome for a treatment with an average order above 3 when compared to a treatment with an average order below 3 . Such would particularly be the case for comparisons of CES-225 and CES-150 with Pla-CES, but not for comparisons between CES-150 and Pla-W. Since many comparisons of CES-225 and CES-150 with Pla-CES yield significant differences, we determined whether these were actually due to order effects. The order difference was correlated with the actual caffeine-placebo difference. If a learning effect had taken place, or the reverse, these correlations, would deviate from zero. We examined all caffeine-placebo comparisons which were significantly different in this way. One comparison (delayed recognition RT CES-225 vs. Pla-CES after exercise) was obviously due to an order effect as expressed by a significant correlation of .74 between order difference and treatment difference. All other comparisons resulted in low and nonsignificant correlation coefficients. "Thts means that differences in treatment orcer could only explain the significant improvement of delayed recognition $\mathrm{RT}^{T}$ after exercise in the CES-225 condition compatred to Pla CES. Thus, with this one exception, treatment order did not influence the results.

Before exercise, subjects had consumed $8 \mathrm{ml} / \mathrm{kg}$ body weight or $587 \mathrm{ml}$ of the test drinks, i.e... they had ingested either water or water containing $40 \mathrm{~g}$ of $\mathrm{CHO}$ and $0 \mathrm{mg}, 88 \mathrm{mg}, 132 \mathrm{mg}$, or $188 \mathrm{mg}$ of caffeine. Before exercise, delayed recall memory performance was significantly improved after CES-150 than after both placebos Thus caffeine improved long-term memory performance. There was no difference between Pla-CES and Pla-W. However, alihough there was no effect of CES alone on memory, the observed memory improvement after CES-150 in comparison with Pla-W could also be attributed to the ingestion of $40 \mathrm{~g}$ carbohydrates and/or to the ingestion of $88 \mathrm{mg}$ caffeine. We do not consider it likely that the other ingredients of the CES which were present in low concentrations, contributed to the observed improvements seen after CES-150 as compared to Pla-W. However, a possible contribution can not be excluded. This amount of carbohydrates has been reported to have a mernory improving effect on its own (Benton \& Owens, 1993). 
The effect of caffeine may dose dependent and specific. In general, it is assumed that caffeine acts by enhancing vigilance and alertness and has no direct effect on memory (Nehlig eit al., 1992). However, the lack of effect of $90 \mathrm{mg}$ caffeine on attentional and speed functions in the present study supports the notion that $90 \mathrm{mg}$ caffeine, which was consurned before the time trial, improved long-tern memory performance directly, without enhancing vigilance and alertness. These results are in agreement with those of most other studies, which seem to conclude that the optimal amount of caffeine for improving memory is $100 \mathrm{mg}$ or less (Lieberman et al., 1987). Howewer, Warburton (1995) found that 75 and $150 \mathrm{mg}$ of caffeine positively affected delayed recall, and Smith et al (1994) found positive effects of $4 \mathrm{mg} / \mathrm{kg}( \pm 260 \mathrm{mg}$ ) on free recall and recognition. Possiblly, the differences in habitual use, differences in state of the subjects or the slight difference in tasks used explain the different results found in the studies. Also, we studied male subjects only. Acute doses ( 2 and $4 \mathrm{mg} / \mathrm{kg}$ ) of caffeine impaired memory performance in females, but often have no such effects in males (Erikson et al., 1985).

By the time they had finished exercise, the subjects had consumed $14 \mathrm{~mL} / \mathrm{kg}$ bodly weight or 1027 $\mathrm{ml}$ of the test drinks, i.e., either $1027 \mathrm{ml}$ water or $1027 \mathrm{ml}$ containing $71 \mathrm{~g}$ of $\mathrm{CHO}$ and $0 \mathrm{mg}$, $154 \mathrm{mg}$, $231 \mathrm{mg}$, or $329 \mathrm{mg}$ caffeine. After exercise, CES-150, when compared with Pla-CES, improved the speed of recognition of words learned before exercise, visual signal-detection efficiency, SCWT performance and Choice and S-R incompatible Choice Reaction Time. The CES-225 drink when compared with Pla-CES improved the speed and quality of signal-detection, SCWT performance and S-R incompatible Choice Reaction Time. The CES-225 drink when compared with Pla-W improved the quality of signal-detection. The CES-320 drink improved SCWT performance when compared with both Pla-CES and Pla-W.

Furthermore, exercise improved perceptual interference, simple RT and S-R incompatible Choice RT. This confirmed findings of an earlier study, in which exercise improved Choice RT and SCWT (Hogervorst et al.s 1996). In the present study, caffeine positively affected most cognitive functions in addition to the effect of exercise. If caffeine improved cognitive performance after exercise by counteracting fatigue, one would expect that cognitive or psychomotor performance would be impaired after exercise. However, this was not the case, since the tasks that require fast response preparation processes, i.e. simple RT, S-R incompatible Choice $R T$ and SCWT interference, were performed better after exercise.

Curiously, a significant difference in cognitive performance after exercise was only observed when the caffeine-containing CES drinks were compared to CES without caffeine. However, when taking Pla-CES the subjects did not show an exerciseminduced increase in speed of performance except on the simple RT. This may indicate that subjects were less activated and aroused after Pla-CES than after Pla-W and the caffeine-containing drinks. Interesting in this respect is the observation that $\mathrm{Pla}-\mathrm{W}$ resulted in the poorest ergometric performance times (Kovacs et al., in preparation).

Physiologically, subjects may have been challenged more by exercise when drinking plain water than when drinking carbohydrates-containing solutions. Generally, CES ingested during exercise reduces physical fatigne and improves physical work capacity compared to water alone (Coyle \& Coggan, 1984). Additionally, the ingestions of CES may blunt the exercise-induced rise in catecholamines and increase plasma insulin levels. Both will reduce lipolysis. Thus, catecholamines and plasma FFA concentrations will be higher after ingestion of water than after ingestion of CES (Brouns, 1989). We indeed found that plasma free fatty acids were significantly higher after Pla-W than after all other test drinks (Kovacs et al., in preparation).

Norepinephrine and epinephrine are also well known stimulants of the central nervous system (Robbins \& Everitt, 1995). As such, their actions may explain the increase in speed of mental 
performance after exercise and could explain the relatively small difference in performance between subjects drinking caffeine-containing drinks and subjects drinking Pla-W.

Our results demonstrate, for the first time, that caffeine enhances cognition before and after strenuous exercise. This is important because the capacity to concentrate effectively is widely regarded in competitive sport as the key to successful performance (Moran, 1996). Our results show that these functions can be improved with a relatively low dose of caffeine in the range of $150-225 \mathrm{mg} / \mathrm{L}$ and that these effects are not further enhanced by increasing the dosage of caffeine (i.e. $320 \mathrm{mg} / \mathrm{L}$ ). 


\section{PART III : HORMONAL REPLACEMENT THERAPY AND COGNITION}

Chapter 10

\section{Hormonal Replacement Therapy and Cognitive Functioning in Elderly Women}

Eef Hogervorst, Wim Riedel, Marjolein Boshuisen, \&elle Jolles

\section{Introduction}

Hormonal Replacement Therapy (HRT) for postmenopausal women has become widespread over the past decades. In Europe, $1-25 \%$ of the postmenopausal women receive HRT to treat postmenopausal symptoms, such as hot flushes, but also as a prophylactic therapy for osteoporosis, and atherosclerosis (Birkhäuser, 1996). For instance, the risk for both fractures and myocardial infarction has been shown to be reduced by $50 \%$ after a minimum of 6 years of HRT (Bottiglioni, 1996). In addition, it has been suggested that HRT improves cognitive functions in elderly normal women (Campbell \& Whitehead, 1977; Fedor-Freyberg, 1977; Hackman \& Galbraith, 1977; Kampen \& Sherwin, 1994; Phillips \& Sherwin, 1992; Sherwin, 1988; Sherwin \& Phillips, 1990; Vanhulle \& Demol, 1976) and reduces the risk of developing Alzheimer"s Disease (AD) and Vascular or Multi-infarct dementia (Lopes, Mensier, Latrent, \& Besse, 1995; Simpkins, Singh, \& Bishop, 1994). Dementia is one of the fastest growing disabling diseases and it is estimated that currently 160.000 patients over 55 years of age suffer from the "silent epidemic" in the Netherlands (Ott ef al., 1996) with annual incidence of 20-25.000 cases (Blom, 1997). Dementia is characterized by severe cognitive dysfunctioning, such as decreased memory and planning functions (Jolles, 1986). The human and economical burden of this diseuse renders more insight into underlying pathological processes and possible therapies necessary.

There are three main questions yet to be answered with regard to the relationship between HRT and cognitive function. The first is concerned with the possible long term protective effect of HRT. Although epidemiological studies seem to indicate a negative relationship with HRT and the development of dementia (Henderson, Paganini-Hill, Emanuel, Dunn, \& Buckwalter, 1994; Mortel, \& Meyer, 1994; Paganini-Hill \& Henderson, 1994; Tang et al., 1996), in a follow-up study of normal elderly subjects, HRT was not seen to prevent cognitive decline (Barrett-Connor \& Kritz-Silverstein, 1993 ).

Second, it is unclear at which behavioral levels HRT exerts its effects and whether this effect is clinically relevant. The experimental studies into the effect of HRT on dementia only involved small numbers of subjects. These studies measured only global changes in cognitive functioning over a relatively short period of time. In normall postmenopausal women, some investigators (e.g. Sherwin, 1994) found specific effects of HRT, i.e. on werbal memory and fine motor skills, but 
not on attentional functions. By contrast, others only found global changes in well being without an effect on memory (Ditkof, Crary, Cristo, \& Lobo, 1991). Methodological issues may account for some of the differences found. For instance, the hormonal preparations tested differed between studies.

For clinical use. HRT usually consists of estrogens combined with progestagens in a second phase to prevent endometrial hyperplasta. In vitro studies have shown that there are many actions through which estrogens may affect brain physiology, e.g. in affecting neurotransmitter synthesis and actwity (Luine, Kthychevskaya \& McEwen, 1975, Luine \& McEwen, 1977; Simpkins et al., 1994); in preserving cholinergic neurons (Janowski \& Rausch, 1985, Rainbow, Davis, \& McEwen, 1980); and in enhancing cerebral perfusion (Funk, Mortel, \& Meyer, 1991). However, progestagens have been found to oppose some of the effects of estrogens in the brain (Sarrel, 1989; Woolley \& McEwen, 1993). The third question is concerned with the mechanism of action of HRT combination therapy. It is at present unclear at which system the combination therapy of estrogens and progestagens is most likely to exert its effect in normal and demented subjects.

In this paper we will discuss the nature of the effect that HRT has on cognitive functions in normal elderly and demented women, in terms of short and long term effects, in specific or global effects, and in terms of the effects of the addition of progestagens. For this purpose $C D$ Rom searches (1966-1997) have been done on HRT. Additional literature has been searched on the basis of references mentioned in articles. Then, all articles were read and evaluated on the basis of the selection of subjects, the diagnostics, the design, the dependent wariables used, statistics, and the conclusions drawn. First, epidemiological case-control and experimental studies are reviewed to find evidence whether HRT is indeed associated with protective actions upon the cognitive functions in demented women. Second, the effects of HRT on cognitive functions and global well being in normal healthy postmenopausal women are discussed. Third, the actions of estrogens and/or progestagens upon different biological mechanisms are described. Lastly, possible biological mechanisms through which HRT may actually act upon cognitive functions and the merit of HRT use on cognitive function are discussed.

\section{Studies into the effects of HRT in demented women}

\subsection{Introduction}

Alzheimer's disease (AD) is a progressive dementing disorder, which is the most prevalent form of dementia, and which is characterized by severe nemory and intellectual disturbances (Jolles, 1986). Henderson and Buckwalter (1994) investigated the finding that women are more likely than men to develop AD. This may be because of their higher life expectancy, since increasing age is a well known risk factor for the development of $\mathrm{AD}$. Howewer, the age-specific prevalence of $\mathrm{AD}$ is also higher in women than in men $(2: 1$ ratio). The authors found that demented women were worse on verbal memory tasks (naming task, verbal fluency, and delayed recally as compared to demented men, while in non demented controls the opposite is observed. This is interesting since verbal memory functions are thought to be specifically very sensitive to estrogen replacement therapy (Sherwin, 1994) and this suggests that estrogens help maintain verbal memory functions in aging women (Sherwin, 1997).

Middle-aged women, as opposed to men, experience a significant alteration in the production of gonadal hormones, ending in a dramatic cessation of the production after the menopause (mean age 51.2 years, SD 3.4 years) (Judd, 1987). It is plausible that an estrogen deficiency or an altered response to estrogens could have a part in the eventual development of postmenopausal $\mathrm{AD}$ in susceptible women. This is illustrated by the findings of Fillit, Ashby, Weinreb, Zabriskie, 
Luine and McEwen (1986a) who compared 12 AD patients with 20 controls (aged $76.7 \pm 3.3$ years) on serum estradiol and total estrogen levells. They found that both variables were significantly lower in patients. Also receptor alterations may play a role. Christie Hunter, Bennie, Wilson, Carroll and Fink (1990) showed that the estrogen stmulated neurophysin challenge was reduced in 10 Alzheimer patients (age $65 \pm 2$ years) as compared to 7 age-matched controls. This means that in AD patients the response to estrogens is also reduced or delayed. Estrogen (ERT) or Hormonal Replacement Therapy (HRT) could then possibly protect against the development of $\mathrm{AD}$, as was suggested by several authors in last years.

\subsection{Case-control srudies of subjects with Alsheiner's disease (AD)}

Several case-control studies have suggested that women afflicted with $\mathrm{AD}$ who used ERT are in a better cognitive state as compared to non users. For instance, a large multicenter study involving 1648 moderate to moderate severe AD patients found that of 970 women, only 89 (9\%) had used ERT. Users had a significant better performance on the ADAS-Cog (corr MMSE - 76), the cognitive subscale of the Alzheimer's Dementia Scale. This effect remained when performance was adjusted for age, education, and age at onset of dementia (Doraiswamy el al., 1997). In another case-control study, the differences between users and non users were investigated in more detail. Henderson, Watt, \& Buckwalter (1996) reported that the nine AD women that were receiving ERT performed better than the 26 mon users on semantic memory (Boston Naming task), short term memory (digit span forward and backward), semantic retrieval (animal fluency), a language comprehension test (Token test), and a visuospatial test (clock and house drawing). Trends were observed for a lower global dementia severity (MMSE and Blessed test of Orientation-Memory-Concentration) in the ERT users (MMSE: $16.2 \pm 6.6$ versus $11.8 \pm 8.3$ ). No differences were seen for verbal episodic memory (10 item word list), nonverbal short term memory, or depression (Geriatric Depression Scale). Subjects had been matched for age, years of education, age at onset of dementia symptoms and duration of dementia symptoms. In this study also performance of $\mathrm{AD}$ men was compared to ERT users and non nsers. However, the former observed gender difference of $\mathrm{AD}$ patients in this study was small and non significant (Henderson, Watt, \& Buckwalter, 1996).

Calse-control studies have suggested that HRT may decrease the risk for developing AD (table 1.). For instance, one study (Mortel and Meyer, 1994) revealed that among 308 women investigated, 94 had Alzheimer"s disease and 65 had vascular dementia. Remaining subjects $(n=149$ ) were taken as controls. Twenty percent of the control group had received ERT as compared to $11 \%$ of the demented group. Mortel and Meyer (1994) concluded that the risk of developing dementiat without HRT was doubled. In another study by Henderson, Paganini, Emanuel, Dunn, and Buckwalter (1994), subjects were included from a volunteer sample recruited from the community. The women were investigated with neurological examination and neurow psychological assessment. 143 women were initially diagnosed with AD (MMSE $7.1 \pm 8.0$ ) and 92 non demented women were included as controls (MMSE $28.8 \pm 1.2$ ). AD patients were lless likely to have received HRT as compared to controls ( $7 \% \mathrm{vs} 18 \%$ ) but did not differ with regard to the use of other medication. In this study the group of subjects with AD receiving HRT $(n=10)$ also had a significantly higher MMSE score (14.9 vs 6.5) as compared to AD patients that were non users $(n=128)$. The groups did not differ in age, education or symptom duration.

A longitudinal follow-up study was done by Tang, Jacobs, Stern, Marder, Schofield, Gurland, Andrews, \& Mayeux (1996). Non-demented elderly randomly diawn from a community were included and a follow-up took place after 1 to 5 years after enrollment. This study showed that 
the Relative Risk (RR) of developing $\mathrm{AD}$ was significantly reduced in women that used $\mathrm{HRT}$. Stratified analyses for age at baseline were done to reduce cohor effects, since AD subjects were older and ERT users were younger. Also, the analyses were adjusted for years of education, sinee $A D$ subjects had less education and HRT users bad more edacation in years. Overall, $12.5 \%$ of the 1124 women had used HRT after the onset of the menopause. Only $5.8 \%$ of the estrogen users as compared to $15 \%$ of non users had developed AD, which was diagnosed by medical records, neuropsychological tests, and imaging techniques. The dwation of HRT was also thought to be important in risk reduction, since the lowest risk was seen in wornen with a history of long term HRT use ( $>1$ year). The authors concluded that HRT did not prevent AD, but delayed the onset of the disivase.

Table 1: Case-control studies dereribing the Odds Ratio (OR) as an estimator of the Relative Risk (RR) in developing $A D$ with $H R T$ inge $(R R<1=$ protective effect)

\begin{tabular}{|c|c|c|c|c|c|c|}
\hline Author (year publ. & Population & $\begin{array}{l}\text { Anformation } \\
\text { on use }\end{array}$ & Age & $\begin{array}{l}\text { Outcome H } \\
\text { HRT : CO }\end{array}$ & $\begin{array}{l}\text { T/AD: } \\
\text { Risk }\end{array}$ & $\mathrm{OR}$ \\
\hline Tang el all (1996) & $n=1124$ & self & $74(\mathrm{SD}=7)$ & $5: 15 \%$ & $\times 3.0$ & 0.40 \\
\hline Henderson (1994) & $t \theta=220$ & self/caregiver & $76(S \mathrm{D}=9)$ & $7: 19 \%$ & $\times 2.7$ & 0.35 \\
\hline Mortel \& Meyer (1994) & $n=308$ & siell" & +73 & $11: 20 \%$ & $\times 1.8$ & 0.48 \\
\hline Kawas et al (1997) & $n=472$ & self & $62(28-94)$ & $27: 50 \%$ & $\times 1.9$ & 0.35 \\
\hline Paganini-Hill et a(1994) & $n=534$ & self & $87(S D=6)$ & $38: 46 \%$ & $\times 1.2$ & 0.69 \\
\hline Broe at al $(\$ 990)$ & $n=340$ & surrogate & $79(\mathrm{SD}=7)$ & $8: 11 \%$ & $x \| .4$ & 0.78 \\
\hline Amadueci et al (1986) & $n=329$ & surrogate & $40-80$ & $10: 13 \%$ & $\times 1.3$ & 0.71 \\
\hline Bremner et al (1994) & $m=227$ & pharmacologist. & $77(S D=7)$ & $50: 50 \%$ & $\times 1.0$ & 1.10 \\
\hline Grives et al (1990) & $n=120$ & surrogate & \pm 65 & $18: 16 \%$ & $\times 1.1$ & 1.15 \\
\hline Heyman et al (1984) & $r y=120$ & surrogate & $61(51-7 i)$ & $15: 7.5 \%$ & $\times .5$ & 2.38 \\
\hline
\end{tabular}

The Baltimore longitudinal study by Kawas et all (1997) inwestigated a sample of post and perimenopausal women $(n=472$ ) with a follow-up for up to 16 years. $45 \%$ of the cohort had used BRT. Thirty-four incident cases of AD (assessed with neurological examination, laboratory, and imaging studies) were reported, which included 9 ERT users. After adjusting for education, the RR for $A D$ in ERT users as compared to non users was .46. No effect was found for the duration of ERT use. However, the authors stated that the sample was not entirely representative of the gemeral population in terms of education (too high), socioeconomic status, and ERT usage (45\%). Paganini-Hill \& Henderson (1994) investigated an upper middle-class cohort of 2529 women, which had died between 1981 and 1992. They identified 136 female cohort members which had SDAT or related diagnoses on their death certificate. For every HRT user, 4 controls were individually matched using birth and death date. Of the women who had AD on their death certificate, 37.5\% had used HRT, of the controls $46 \%$ had used HRT. The relative risk was .69 for women using HRT to develop SDAT. The risk for AD decreased with a longer duration of use. Other medication or drug use was not associated with an increased risk and the Odds Ratio (OR) as an estimator of the Relative Risk (RR) remained essentially unchanged when adjusted for age, type of menopause (surgical natural), medication use, blood pressure, or stroke. However, in this study, education was not controlled for. 
Several studies did not find any prophylactic effect of estrogens. For instance, Brenner et al. (1994) did not find any evidence that ERT reduced the risk of AD. Brenner et al obtained information on ERT use from computerized files of pharmacists. The AD diagnosis $(n=107)$ was made using a standardized examination including medical history, physical and neurological examination, and neuropsychological and laboratory tests, including imaging techniques. Control subjects $(n=120$, MMSE $>28$ ) were selected through random sampling of the same survey population and matched for age. Controls were also investigated psychometrically and clinically (chart review and judgement of a nurse). About $50 \%$ of cases and controls had received HRT. No association of HRT with AD could be found. Although controls had more years of education as compared to the cases, education, smoking, nor progestagen use was found to confound results. In the study by Amaducci et al (1986) $\mathrm{AD}$ was assessed using a standardized protocol including the Blessed Dementia Scale, the Hamilton Depression Scale, clinical history, neurological examination, and neuropsychological and laboratory tests to exclude dementias other than AD. For each patient $(n=116)$ one hospital and one population control (an acquitance of the patient) was matched for age and sex. Thus, in this study both men and women were included. No association (OR) between ERT and AD was found. Simillarly, a study by Broe et all (1990) found a non significant association (OR) between hormonal treatment and $\mathrm{AD}$. AD was assessed by standardized clinical examination including assessment by a neurologist, history taking, MMSE, and neuropsychological and laboratory tests. 4 controls (MMSE $>26$ ) matched for age and sex were contacted by the referring general practice. 'Hormonal treatment' was assessed through surrogate responders but it is unclear whether this concerned HRT specifically or anticontraceptive use in general. Weak differences in the opposite direction were reported in a study by Graves et al (1990) who included 60 AD patients clinically diagnosed (MMSE: $16.3 \pm$ 7.0) and 60 controls (friends with a MMSE > 26). Information on HRT use was obtained from surrogate responders by telephone. $16.3 \%$ of the controls had used HRT, while $17.6 \%$ of the patients were HRT users. Patients were older than controls (66.2 versus 63.6), but age was adjusted for in the risk factor analyses. In this study also both males and females were included. Lastly, a remarkable result was reported by Heyman, Wilkinson, Stafford, Helms, Sigmon and Weinberg (1984) who found that the use of ERT in AD patients was twice as thigh as compared to controls ( 15 vs $7.5 \%$ ). Howewer, the OR was nonsignificant. In this study 12 male and 28 female AD patients were included. Por each case two controls (MMSE $>20$ ) were at random selected by telephone sampling and matched for sex and age.

\subsection{Case-control studies of subjects with cerebrovascular disease (CVD)}

The second most prevalent dementing disease is vascular or multi-infarct dementia. It is possible that HRT exerts prophylactic effects on vascular dementia as well. In a longitudinal case-control study of 158 women (aged $40 \mathrm{~m} 90 \mathrm{yrs}$ ), women with a history of cerebrovascular disease (CVD; $n=51)$ were compared to controls $(n=107)$. Of the HRT users $(n=46) 16(35 \%)$ had had $C V D$ such as Transient Ischemic Attacks (TIA) or Reversible Ischemic Neurologic Deficits (RIND), while of the non HRT users, 35 subjects (31\%) had had TIA or RIND. However, the subjects with CVD which received HRT did not show the cognitive decline after 18 months as compared to non users with CVD on the Cognition Capacity Screening Examination. This is a test which, according to the authors, has a good correlation with the MMSE. There was also a non significant trend for an increased cerebral perfusion among subjects with CVD receiving HRT. HRT was hypothesized to positively influence cognitive function by helping to sustain cerebral perfusion (Funk et al., 1991). A study by Schmidt et al (1996) investigated the effect of ERT use in preventing silent 
ischemic brain damage, operationalized as white matter hyperdensity areas on MRI. 70 ERT users were compared with 140 non users, matched for age (on average $60 \pm 6.0$ ). Users were better educated and were less likely to have arterial hypertension. Also, a trend was seen towards a lower mean arterial blood pressure in users. Subjecively, users reported a lower activation. ANCOVAs were used to correct for slight differences in age, education, mean arterial blood pressure, and self-reported activation, of 18 neuropsychological parameters, only 2 (after adjusting for multiple comparisons) were stgnificant: Users were better at the Wisconsin Card Sorting Test (5.8 versus 5.4 categories completed conceptual reasoning) and on the Purdue Pegboard test (visuopratical skills). No differences were found in werbal- (e.g. story recall) and visuomotor memory (e.g, recall of designs), attention and speed (Alters-Konzentrations-Test, TMT-B, Digit $\mathrm{Span}$, complex RT lasks) tests. Women that used ERT had a lower rate and extent of white matter hyperintensity areas, which was inversely related to the duration of ERT use. However, there was no association between neurological performance and the extent of white matter disease.

\subsection{Experimental studies with demented subjects}

In experimental designs with pre-and posttests, the favorable effects of estrogens on cognitive functions in subjects with dementia of the Alzheimer type have been described (table 2). Fillit, Weinreb, Cholst, Luine, McEwen, Amador, and Zabriskie (1986) reported the results of a trial of 6 weeks of ERT, which showed that a low dose of estradiol $(2 \mathrm{mg} /$ day $)$ had a significant positive influence on social behavior, orientation, and attention/short term memory (STM) functions in 3 out of 7 women with Alzheimer's Disease. Responders had an older age at onset of dementia (72 versus 61 in non responders), a higher baseline MMSE (median score 14 versus 3.5 ) and a lower baseline Global Deterioration score. In a later crossover study Fillet (1994) reported no effect of ERT on the MMSE, paired associate learning or affect (Bradburn Affect Balance Scale) in 8 elderly women with Alzheimer's Disease. The author concluded that the dosage (transdermal patch of $0.05 \mathrm{mg}$ of estradiol) may have been too low. Honjo, Ogino, Naitoh, Urabe, Kitawaki, Yasuda, Yamamoto, Ishikara, Okada, Yonezawa, Hayashi and Nambara (1989) treated 7 women with SDAT with conjugated estrogens or estrone sulfate over a 6 week period, and tested them every 3 weeks. Six women showed improvements in the New Screening Test for Dementia (NS: memory, orientation, and calculation) and 5 showed improvements in Hasegawa Dementia Scale (HDS). Withdrawal bleeding occurred in 5 out of 7 women. Seven untreated SDAT patients and 7 thealthy controls did not show improvements in cognitive function (Honjo et al., 1989). In a study by Olikura, Isse, Akszawa, Hamamoto, Yaoi and Hagino (1994a) 15 AD patients were treated with $0.625 \mathrm{mg}$ of conjugated estrogens for 6 weeks. 4 were diagnosed as mild, 7 as moderate and 4 as severly demented. Mean Mini Mental State Examination (MMSE) score increased significantly as compared to baseline in 10 out of 15 patients, HDS score increased in 11 out of 15 patients. Changes mainly occurred in the mild to moderate demented group. No changes occurred in an untreated control group $(\mathrm{N}=15)$. Subjects using ERT returned to baseline levels at three weeks after termination of ERT. In the patients treated with ERT, the regional cerebral blood flow (CBF) increased significantly in the lower frontal region and primary motor area of the right hemisphere. The increase in CBF in the frontal region was hypothesized to be associated with the increase in functioning of the cholinergic neurons of the nucleus basalis of Meynert. Pathological signs on EEG (mean absolute power delta and theta band values) were also decreased significantly in ERT users (Ohkura et al., 1994a).

Ohkura, Isse, Akezawa, Hamamoto, Yaoi and Hagino (1994b) also presented a longer study of 10 mild to moderate $A D$ patients (MMSE $18.8 \pm 1.7$ ) which received conjugated estrogens (0.625 $\mathrm{mg} /$ day) for 5 months in cycles of 21 days and were compared to 10 untreated Alzheimer control 
subjects (MMSE 18.0 \pm 1.3 ). A slowed rate of decline on cognitive functions as measured with the MMSE was seen in the ERT group $(20.5 \pm 2.0)$ as compared to controls $(15.2 \pm 1.5)$ but only after five months of treatment. Two subjects in the ERT group experienced withdrawal bleeding (Ohkura et al., 1994b). Another longer duration study by Ohkura, Isse, Akazawa, Hamamoto, Yaoi, and Hagino (1995) included 7 female patients with mild to moderate dementis of the Alzheimer type who were treated with long term low dose ERT for at least 5 months. MMSE and the HDS scores were elevated above pretreatment levels during HRT in 4 out of 7 patients, 2 patients responded moderately well and one did not respond at all. Termination of HRT resulted in a decrease in scores. The women $(n=4)$ that used additional progestagens (after day 12) showed a physical poor condition and/or appeared subdued during this phase (Ohkura at al., 1995).

The only study with $\mathrm{AD}$ patients that employed a double blind placebo controlled design was done by Honjo, Ogino, Tanaka et al (1993). In this study, 14 AD patients were assigned at random to either $1.25 \mathrm{mg}$ of conjugated estrogens (CEE, Premarin) or placebo for 7 weeks. Progestagens (2.5 mg MPA) were added to CEE at weeks 4.7 . The 7 women using ERT showed improvements on the MMSE (from $18.0 \pm 5.6$ to $21.7 \pm 4.2$ ), the HDS and the NSD (Iapanese National Test for Dementia) after three weeks as compared to baseline, while controls showed no significant change (MMSE from $17.2 \pm 6.8$ to $18.1 \pm 6.2$ ). On the HDS and the MMSE significant improvement was seen on immediate memory. However, between subjects comparisons were only significant for the HDS. Scores were slightly decreased in the sixth as compared to the third week, when MPA was added, which suggests that the addition of a progestagen has detrimental effects on cognitive function. Breakthrough bleeding occurred in 8 patient 5 and withdrawal bleeding occurred in 3 patients. Uterine bleeding thus occurred in $85 \%(11 / 13)$ of the patients (Honjo et al., 1993).

Table 2: Expermental studies, with the effect of HRT in women with Alzheimer"s disease

\begin{tabular}{|c|c|c|c|c|c|}
\hline Name & Stubjects & Controls & Age & Treatment & Outcome \\
\hline $\begin{array}{l}\text { Fillet at al } \\
(1986)\end{array}$ & 10 SDAT & - & $\begin{array}{l}66.5 \\
(5.0)\end{array}$ & $\begin{array}{l}3 \mathrm{mg} \text { sstradiol } \\
6 \text { weeks }\end{array}$ & $\begin{array}{l}\text { 3:7improved soctal behaviot } \\
\text { orientation, atcention/STM }\end{array}$ \\
\hline $\begin{array}{l}\text { Fillet } \\
(1994)\end{array}$ & $8 \mathrm{SDA}$ & crossower & $?$ & $\begin{array}{l}0.05 \text { mges estrat } \\
3 \text { manths }\end{array}$ & $\begin{array}{l}\text { foro improvernent MMSE, menory } \\
\text { or affect }\end{array}$ \\
\hline $\begin{array}{l}\text { Honjo et al } \\
\text { (1989) }\end{array}$ & $7 \mathrm{SDAT}$ & $\begin{array}{l}7 \mathrm{SDAT} \\
7 \text { non dem }\end{array}$ & $\begin{array}{l}80.1 \\
(2.9)\end{array}$ & $\begin{array}{l}1.25 \mathrm{mg} \mathrm{CEL} \\
6 \text { weeks }\end{array}$ & $\begin{array}{l}\text { 6: innoroved NS, } \\
\text { 5: improved HDS }\end{array}$ \\
\hline $\begin{array}{l}\text { Honjo et al } \\
\text { (1993) }\end{array}$ & $7 \mathrm{SDAT}$ & $7 \mathrm{SDAT}$ & $\begin{array}{l}83.7 \\
(4.5)\end{array}$ & $\begin{array}{l}1.25 \text { mg CEEA } \\
\text { placebo } 3 \text { whs }\end{array}$ & $\begin{array}{l}7 \text { on CEE mprowed on MMSE } \\
\text { NSD, \& HDS, none on platebe }\end{array}$ \\
\hline $\begin{array}{l}\text { Ohkura et } \\
(1994 a)\end{array}$ & $15 \mathrm{SDAl}$ & $\begin{array}{l}15 \mathrm{SDAT} \\
\text { unureated }\end{array}$ & $\begin{array}{l}71.9 \\
(2.4)\end{array}$ & $\begin{array}{l}0.625 \mathrm{CEE} \\
6 \text { welks }\end{array}$ & $\begin{array}{l}\text { 10: improwed MMSE: } \\
\text { 11: improved HDS }\end{array}$ \\
\hline $\begin{array}{l}\text { Ohlkura el } \\
\text { (199.4b) }\end{array}$ & $10 \mathrm{SDAT}$ & $\begin{array}{l}10 \text { SDAT } \\
\text { unimeated }\end{array}$ & $\begin{array}{l}70.7 \\
(2.1)\end{array}$ & $\begin{array}{l}0.625 \mathrm{ngg} \text { CEE } \\
5 \text { months }\end{array}$ & $\begin{array}{l}\text { MMSE ERT }>\text { CON, HOS ERT } \\
>\text { CON reewll, recent ewents? }\end{array}$ \\
\hline $\begin{array}{l}\text { Ohkurat al } \\
(1995)\end{array}$ & 7 SDAT & - & $56-77$ & $\begin{array}{l}0.625 \mathrm{mg} \mathrm{CEE} \\
5 \mathrm{mth}(\mathrm{MPA} 4)\end{array}$ & $\begin{array}{l}47 \text { improved MMSE \& HDS } \\
2 \text { moderite respontse }\end{array}$ \\
\hline
\end{tabular}

Two studies evaluated the effect of ERT when combined with a potential cognition enthancer. The study by Weiss (1987) treated 5 women with moderate AD (MMSE 12-23) with estradiol (1 mg) for 14 days, combined with nalmefene, an opiate antagonist, from day 15 to 22 . An extensive 
battery was adminstered weeklly (e.g. GDS, MMSE, Hamilton Dep Scale, Buschke Selective Reminding, TMT, DSST, Fluency). One patient dropped out, and three showed no improvement while taking estradiol alone or when combined with nalmefene. The other patient deteriorated until was discontinued after 28 days and then reversed to baseline. However, in this study only few patients were examined within a very short period of only 14 days. Schneider, Farlow, Henderson, \& Pogoda (1996) had a different approach and evaluated the effect of current ERT ase (13\% of the 118 mild to moderate $\mathrm{AD}$ patients) on the clinical and cognitive response to tacrine. ERT users were younger (on average $67 \pm 8$ versus $74 \pm 8$ years) and better educated. Both alge and education were adjusted for with ANCOVA. Results show that women which used ERT and tacrime had a better response at 30 weeks on the ADAS-Cog $(\mathrm{p}=.02)$ than women that did not receive ERT and who were assigned to a placebo or tacrine. Similar trends were seen for the MMSE. The duration of ERT usage (mean 11 years) was not related to outcome. Non use of progestagens (use in 8 patients) was also independently related to a better outcome on the ADASCog. The figures suggested that tacrine alone took an intermediate position between placebo and tacrine with ERT. However, after a peak at 24 weeks, there appeared to be a trend for a slight reversal of effects at week 30 . Also, since ERT use was non randomized, differences in health care, the higher level of education, and the younger age of ERT users may be potentiall sources of bias. Although these factors were controlled for with ANCOVA, it was not clear how these factors were adjusted (adjusted means ?) for using two sample t-tests for pairwise comparisons between placebo and treatment groups. Furthermore, it should be kept in mind that among treatment completers only 8 subjects were left receiving ERT and tacrine versus 50 on tacrine alone and 50 on placebo (Schneider, Farlow, Henderson, \& Pogada, 1996), which increases the riisk for chance findings.

\subsection{Discussion and methodological considerations}

Controversial effects of HRT on AD have thus been found. Different effects may be attributed to methodological issues. First, important is that most of the studies mentioned were not based on randomly drawn population samples. The large variation in the proportion of HRT users (12.5$50 \%$ ) points to differences im sampling. This is important since a large number of non users may confound effects (Sackett; Haynes, Gyuatt, \& Tugwell, 1991). Second, in the study by Paganini-Hill \& Henderson (1994), death certificates after an 11 year follow up period were used for the diagnosis of SDAT. The use of death certificates did not wrant adequate diagnosis, ats was reflected by the nomenclature of the disease: "Alzheimer's Disease", "Senility", "Senile dementia' or 'Dementia'. Other studies, including the studies that did not find effects, had extensive diagnostic methods. Differences in the methods and criteria to classify SDAT may have caused different findings.

Third, the HRT use (and number of years of use) in the study by Tang et al (1996) was assessed by self report. Retrospective self-reports are known not to be very relliable and not a valid reflection of actual facts (Sackett, Haynes, Guyatt, \& Tugwell, 1991). In the study by Tang, the selfreport was suspected especially because the duration of use varied considerably, ranging from 2 months to 49 years. Paganini-Hill \& Henderson (1994) also obtained information on use at entry in the study by siubjects themselves. In the study by Amaducci et al (1986), information on HRT use was obtained by spouses or family (so called 'surrogate responders'). This may cause even a larger information bias, since spouses can certainly be questioned to be an adequate source of information of this kind. This may also have been the source of bias in the studly of Heyman, Wilkinson, Stafford, Helms, Sigmon and Weinberg (1984) in which similarly no significant differences in use of HRT between $\mathrm{AD}$ patients $(15 \%)$ and controls $(7.5 \%)$ were 
found. Likewise, in the study by Henderson et al (1994) information on HRT use in AD subjects was received from surrogate responders, while control subjects reported on HRT use themselves. Fourth, in the Paganini-Hill study, no information was awailable on the level of education or the use of other (e.g. antihypertensive) medication. It has been described, for instance, in the study by Tang et al (1996) that subjects with a low level of education often do not to use HRT. Since Alzheimer's subjects also have been found to have had less years of education, the correlation between education, HRT and Alzheimer may confound results. For instance, in the study by Brenner, Kukull, Stergachis, van Belle, Bowen, McCormick, Teri and Larson (1994) which reported no effects of HRT on $\mathrm{AD}$, there were more highly educated subjects in the control group (61\% vs $36 \%$ ) and age was significantly different between groups. However, in the analyses, the difference in level of education was not controlled for. By contrast, in the study by Heyman, Wilkinson, Stafford, Helms, Sigmon and Weinberg (1984) AD patients had a significantly higher level of education, a higher social economic status, and healthier lifestyles as compared to controls. It is remarkable that this (Heyman et al, 1984) study was the only one to report a more than twofold increase in Odds Ratio where all the other studies apart from the studies by Brenner et all (1994) and Graves et al (1990) reported decreases. Since in most other studies (e.g. Henderson et al, 1994; Tang et $\mathrm{al}, 1996) \mathrm{AD}$ patients are found to have had lower levels of education, this may indicate that the controversial results of Heyman et al (1984) could be attributed to a sampling bias.

Also, in the Heyman study, patients of both sexes were included, i.e. 12 male and 28 female AD patients. For each case two controls were at random selected by telephone sampling. Controls were matched for sex and 5-year age interval. This means that only 28 female patients were compared to 56 controls. In each group approximately $4-6$ subjects had received HRT. This small sample size may have increased the risk for chance findings. In fact, the studies that did not find a significant decrease in the OR of $\mathrm{AD}$ (Amaducci et al, 1986; Broe et al, 1990; Heyman et al, 1984) followed this procedure in which cases were pairwise matched with controls. Due to this procedure, some subjects may be lost for analyses which may increase the chance for error findings.

Fifth, women often start using HRT at menopause (51.4 (SD 3.8) years of age) and on average use HRT for approximately 6-13.6 years (e.g. Tang et al, 1996). Late onsel Alzheimer" disease, which is most common in the majority of cases, has an onset above 65 year (APA, 1994). In the years between having used HRT and developing Alzheiner's. Disease, other intervening variables assaciated with age, such as an increased use of other medication liable to induce cognitive deficiency or an increase of the number of narcosis that subjects have underwent, may have come into play. Still, in two of the studies mentioned age and education (Henderson et al, 1994; Tang et al, 1996) and medication (Henderson et al, 1994) were adjusted for in statistical analyses and still prophylactic effects of estrogens were seen. Yet, according to Tang et al (1996) there may still have been other life style characteristics or bias that accounted for the effect observed.

Sixth, although Mortel and Meyer (1994) concluded that the risk of developing dementia without HRT was doubled, an observed association is not by definition an indication of a causal relation. If two variables co-vary, one cannot imply that the two variables are causally related. Henderson, Paganini, Emanuel, Dunn, and Buckwalter (1994) for instance, hypothesized that HRT may be considered less important for $\mathrm{AD}$ patients and therefore be less commonly prescribed. The possible bias mentioned of HRT not being prescribed in AD patients may indeed occur in retrospective cross sectional case control studies. However, this risk is reduced in longitudinal case control studies, such as the study done by Tang et al (1996) and Paganini-Hill \& Henderson 
(1994) in which information on HRT use was obtained apon entry into the study. Lastly. controversial results may point towards a publication bias. Sommer (1982) stated that studies (e.g. into premenstrual cognitive dysfunction) with positiwe findings are more likely to be published than studies with negative or nil findings (Sommer, 1982). This habit results in an increased risk of the type I error.

In sum, as was stated, an observed association is not by definition an indicator of a causal relation. However, evidence for a causal relation between estrogen use and protection of cognitive functioning can be assessed with epidemiological rules of evidence for causation (Sackett, Haynes, Guyatt, \&ugwell, 1991). One of these rules is concerned with the biological plausibility of an effect. Biological plausibility can be derived from in vitro animal studies which supply evidence for mechanisms of action of estrogens in the brain (see par. 4). Another rule is concerned with the evidence derived from experimental studies and related experimental areas. However, witly respect to the data obtained in experimental studies (par. 2.4.), the results should be carefully interpreted. Only mall heterogenous groups of patients $(n=7-15)$ were regarded without blinded controls (except for the study by Honjo et al, 1993). The subject of learning effects, which can be quite substantial in some subjects but not others, is not addressed. This is a problem when tests are not available in parallel versions and when only small groups are tested. A degres of improvement from baseline is often seen to occur in placebo studies, which is a nonspecific response based on the attention given to patients during a study. This can be a risk especially when the investigator is not blinded to the condition. Furthermore, the 3-6 week duration of treatiment (e.g. Fillet et al, 1986; Honjo et al, 1989; 1993; Ohkura et al, 1994a) may be too short to evaluate substantial improvement. For instance, one study only showed effects of ERT after 5 months of treatment (Ohkura et al, 1994b) Also, the NS, HDS, and MMSE are not very complex tests for dementia and may not be the best choice for the evaluation of individual symptoms in AD. Lastly, some of the studies suggest that the effect of ERT is most apparent in mild to moderate dementia (e.g Fillet, 1986; Ohkura et al, 1994a), which narrows down the indication for ERT use in dementia.

By and all, these methodological considerations hamper clear cut conclusions. Additional evidence from studies with normal subjects needs to be considered. For instance, the first two studies documented into the effect of ERT on cognitive impaired elderly women did have randomized, placebo-controlled, longitudinal designs. Although it is unclear whether the women, which were residents of homes for the aged, actually were inflicted with SDAT, their baseline psychometric scores do suggest a significant cognitive impaiment similar to that of AD patients. In the study by Caldwell (1952) 13 of 28 women were given a weekly injection of $2 \mathrm{mg}$ of estradiol over 18 months. After 6 monihs $20 \mathrm{mg}$ of testosterone propionate was added to reduce uterine bleeding. Significant improvement was seen in verbal $\mathbb{I Q}$, comprehension, and the memory subscale of the Wechsler-Bellevue Intelligence Scale after 12 months of treatment. After this periad, performance either reached a plateau or declined. Subjects on placebo showed a progressive decline in performance over the 18-month period (Caldwell \& Watson, 1952). Kantor, Michael, and Shore (1973) gave 50 women at random either $0.625 \mathrm{mg}$ of conjugated estrogens. (CEE, Premarin) or placebo from day 1 to 25 each month for 3 years. Between the sixth and 19th month of treatment, scores on the Hospital Adjustment Scale (scored by blinded nurses) were significantly elevated above baseline with a peak at 12 months. Then sciores declined to the scores observed in the placebo group. None of the women experienced withdrawal bleeding. Hence, these findings suggest that estrogens may improve cognitive function up to 12 months after which a decline or plateau is seen. 


\section{Studies with normal postmenopausal women}

\subsection{Introduction}

Bungay et al (1980) performed a study by means of postal questionnaires in a population sample of 1120 women and 510 men. Minor mental problems, such as difficulty in making decisions, forgetfulness, difficulty in concentration and loss of confidence were shown to peak just before the mean age of the menopause (51.4 years, stdev. 3.8) (Bungay, Vessey, M McPherson, 1980 ). The female ovary secretes $95 \%$ of the estradiol that enters the circulation. From the age of 40 , the aging ovaries produce less estradiol, which is followed by a rapid decline in estrogen levels when the ovaries have become depleted around the average age of 51 (Nudd, 1987). Consequently, about $80 \%$ of the women experience negative consequences of the menopause, of which hot flushes and night sweating are probably the most common symptoms (Utian \& Schiff, 1994). Other symptoms, for which ERT may be indicated are urogenital atrophy, osteoporosis" arteriosclerosis, and complaints of decreased cognitive function and mood (Kauppila, 1996),

Halbreich, Lumley, Palter, Manning \& Gengo (1995) found that there was a significant invesre correlation between age and performance on a number of tests in postmenopausal ( $n=33$, age: $52.1 \pm 4.0 \mathrm{yrs}$ ) but not in young women ( $\mathrm{n}=24$, age: $34 \pm 9.4 \mathrm{yrs}$ ), including driving simulation, a psychomotor test (RT and recognition), and some visuospatial tests, but not on the manual dexterity, the Digit Symbol Substitution Test (DSST), the Trial Making Test (TMT part B), short term memory (Benton"s Visual memory, Bushke's tests), or abstract reasoning (Category test).

It was concluded that detrioration of some cognitive functions is notably accelerated following menopause (Halbreich et al., 1995a).

\subsection{Case-control studies of HRT with normal subjects.}

As contrasted to the findings with demented subjects, not much evidence is given for prophylactic effects of estrogens in the normal population (table 3). For instance, a 15 year prospective, cross sectional study (Barrett-Connor \& Kritz-Silverstein, 1993) did not find that hormonal use preserved cognitive function in normal elderly women. In this study, 800 women (aged 65-95 years) were investigated. Hormonal use was evaluated at baseline $(1972 / 1974)$ and at a follow-up 16 years later (1988/1991). Almost half of the cohort had used estrogen (mostly unopposed conjugated estrogens, Premarin) at some time after the menopause and one third were current users. Age-related cognitive decline was similar for all women even when adjusted for age and education. However, women that had ased estrogens for at least 20 years had higher scores on the animal Fluency test, a test for the retrieval of information from memory. No significant differences occurred in any of the other verbal or wisual memory tests (MMSE, Buschke Selective Reminding Test, Visual Reproduction Test of the Wechsler Memory Scale, Animal Flluency, Trail $\mathrm{B}$ of the TMT of the Halstead-Reitan test battery, and the Blessed Information MemoryConcentration Test). In this study a large number of statisticall (multiple regression) analyses was done, with only a few statistically significant results, less even than expected to occur on basis of coincidence alone.

Another longitudinal study into ERT use, atherosclerotic risk and cognitive function by Szklo et a $\llbracket$ (1996) examined 61110 women with a follow-up after 3 years when the cohor was aged $48-67$ years. At that time, $78 \%$ of the cohort was postmenopausal. Women were excluded with a history of stroke or TIA or when they took antipsychotic medication. Current ERT users (18\% natural menopausal, $49 \%$ surgical menopausal), were younger, better educated, more often married, and more likely to perceive themselves in excellent or good health. They were less frequently 
hypertensive or diabetic, had a lower mean fubrogen level, a lower body mass index, and lower depression scores. There were no consistent associations between ERT use and delayed word recall or between ERT wse and the performance of the Digit Symbol Substitution test (DSST) after adjusting for age, education, and the additional (e.g. atherosclenotic risk) covariates found to be different between users and non users. However, among the women aged 48-57 years, adjusted mean Word Fluency seores were slightly (2.4 word) higher in current as compared to women that had never used ERT. Within current users adjusted Word Fluency scores increased with duration of ERT use. No significant differences were found for the women that were in the age-group of 58 to 67 years. When depressive scores were controlled for results remained virtually unchanged. However, results were in general weak (only $4 \%$ of 24 comparisons reached significance). The authors hypothesized that the duration of ERT use may have been too short or the population may have been to young to experience any substantial cognitive decline (Szklo et al., 1996).

An alternative explanation is that effects of ERT are only apparent in relatively young subjects. In this line, Kimura (1995) included 21 postmenopatusal women ( \pm 58 years of age) on ERT (mainly CEE) and 33 controls, equivalent in age, education, years since menopause, and vocabulary score. Users were tested on and off therapy and the mean of the two scores (converted to a standard $z$ score) was taken as dependent variable. Overall, women on ERT were better on cognitive tests (e.g. Verbal fluency, Perceptual speed, Spatial, Articulatory/motor tests), but no effect was seen on the Profile of Mood States (POMS) (Kimura, 1995). However, since different methods and statistics were used in this study, comparison is difficult.

Table 3: Case-contral studies describing the effect of HRT use on cognitiwe functions in the normal population

\begin{tabular}{|c|c|c|c|}
\hline Author, year of pubi & Subject (mean age, SD) & Tests & Outcome \\
\hline $\begin{array}{l}\text { Barret \& Kritz, } \\
1993\end{array}$ & $\begin{array}{l}n=800,65.95 \text { yrs } \\
\text { (mean age: } 76.9 \pm 6.7 \text { ) }\end{array}$ & $\begin{array}{l}\text { Memory, attentional, } \\
\text { and wisuospatial tests }\end{array}$ & mo effect of HRT \\
\hline $\begin{array}{l}\text { Saklo et al, } \\
1996\end{array}$ & $\begin{array}{l}n=6110,45-64 \text { yrs } \\
\text { (nedun } 6 \mathrm{ge}: 57+5.6 \text { ) }\end{array}$ & $\begin{array}{l}\text { Delayed recall, DSST } \\
\text { Fluency }\end{array}$ & $\begin{array}{l}\text { no ffiect of HRT, } \\
\text { only in young group }\end{array}$ \\
\hline $\begin{array}{l}\text { Robinson et al } \\
1994\end{array}$ & $\begin{array}{l}n=144, \text { matched groups } \\
\text { (nuean age: } 67 \pm 6.5 \text { ) }\end{array}$ & $\begin{array}{l}\text { Object and Name recall } \\
\text { memory test }\end{array}$ & $\begin{array}{l}\text { only eflect HRT on } \\
\text { Name recall test }\end{array}$ \\
\hline $\begin{array}{l}\text { Kampen sherwin. } \\
1994\end{array}$ & $\begin{array}{l}\text { no7l. (2l HRT) } \\
\text { (thetn age: } 65 \pm 5 \text { yes) }\end{array}$ & $\begin{array}{l}\text { Mernory, language, } \\
\text { väsuospatial }{ }_{\text {, attention }}\end{array}$ & $\begin{array}{l}\text { only effect HRT on } \\
1 \text { of } 14 \text { tests }\end{array}$ \\
\hline $\begin{array}{l}\text { Kimura, } \\
1995\end{array}$ & $\begin{array}{l}n=54(2) \text { HRT) } \\
\text { (mean age } 58 \text { yrs) }\end{array}$ & $\begin{array}{l}\text { end Verbal fluency. } \\
\text { Perceptual Speed }\end{array}$ & $\begin{array}{l}\text { overall effect of HRT } \\
\text { on cognition }\end{array}$ \\
\hline
\end{tabular}

Another explanation is that performance of elder women that take ERT shows a larger wariance in performance in some but not on other tasks, which is the reason why significant differences do not become apparent between groups on some tasks. For instance, a cross sectional study by Robinson, Friedman, Marcus, Tinklenberg, \& Yesavage (1994) investigated a group of 278 healthy women (55-93 yrs of age), of which 72 HRT users were matched with 72 non users for age and education. This study revealed that HRT users (on average aged $67 \pm 6.5$ years of age) were better at Name recall but not at Object recall. In this study there was a significant greater variance in the women taking estrogens as compared to controls. It was hypothesized that this caused the nonsignificant findings for Object recall since slight mean differences between groups were apparent. Similarly, a cross sectional case control study by Kampen and Sherwin (1994) revealed that performance of elder women taking ERT (on average aged $65 \pm 5$ years) on immediate and delayed paragraph recall (WAIS) was significantly higher compared to non users. 
However, no differences were found on immediate or delayed word recall (Paired Associates, Selective Reminding test:12 item word list), language skills, spatial memory and skills, and attention. No sociodemographic differences (age, education in years, marital status, socioeconomic status and age of menopause) had been detected between groups. The authors concluded that the effect of estrogens is specific and not global (Kampen \& Sherwin, 1994). Alternatively, the variance on some tasks may have been too large within groups to reveal significant differences between groups or otherwise the many statistical comparisons that were done may indicate that these findings are associated with chance.

\subsection{Experimental studies with posimenopausal women}

Many experimental studies have shown positive effects of HRT on cognitive functions (both subjective reports and objectiwe tests) in natural and surgical postmenopausal women (Campbell \& Whitehead, 1977; Fedor-Freyberg, 1977; Hackman \& Galbraith, 1977; Sherwin, 1988; Sherwin \& Philips, 1990; Sherwin, 1991; Philips and Sherwin, 1992a; Vanhulle \& Demol, 1976, see table 4). For instance, an early study by Düker (1957) into the effects of estrogen therapy revealed less cognitive complaints after the prescription of estrogens in both men and women of middle-age. After the use was discontimued, complaints increased again. By definition, estrogens may thus act as cognition enhancers.

However, Rauramo et al (1975) found no effect of HRT on cognitive functioning in postmenopausal women. Effects of HRT were evident on neurovegetative symptoms, such as perspiration, flushes, tachycardia and dyspnoea. Also, HRT subjects had a better mood (less tearful and irritable) as compared to the placebo group (Rauramo, Lagerspetz, Engblom, \& Punnonen, 1975). Yet, in this study no effects were found on logical reasoning, as measured with the Raven's Progressive Matrices; on reaction times (RT) of letter cancellation, and on the Integrative Memory Test. Parallel groups were employed with measurements after a 1 and a 6 month period. However, the plots reveal that learning effects may have confounded results. Also, both the scales $(0-180)$ used in this study as well as the cognitive tests may be questioned of their validity for the use in these studies. Hackman and Gailbraith (1977) reported subjectively improved memory of a 6 month $1.5 \mathrm{mg}$ piperazine oestrone sulphate treatment against placebo, which was not reflected in objective memory performance (Guild memory test) improvement. Marked improvement was seen in wasomotor (flushes) and other postmenopausal symptoms (insomnia, vaginal discomfort, dyspareunia and urinary symptoms). Withdrawal bleeding occurred in 3 of the 9 patients taken ERT. The authors suggested that the non significant improvements on memory functions may be explained by an overall improvement on mood (Hackman \& Galbraith, 1977). Other authors have also described global improvements after HRT. For instance, the early 12 month double blind crossover study by Campbell \& Whitehead (1977) showed that ERT (for 6 months) improved vasomotor symptoms, vaginal dryness, insomnia, urinary frequency, and subjective memory as compared to placebo. The Beck Depression Score, the General health Questionnaire (GHQ), and neuroticism (EPD) improved for both premarin and placebo similarly. About half of the menopausal and all of the perimenopausal women experienced withdrawal bleeding. Howewer, the higher dosage of 1.25 mg caused endometrial hyperplasia in $28 \%$ of the patients. This could be reversed by a reduction of the dosage or by the addition of a progestagen. Similar effects were reported by Wiklund, Berg, Hammar, Karlberg, Lindberg, \& Sandin (1992) who found that estraidiol over 12 weeks improved health related quality of life. However, also the placebo group was seen to improve (to a lesser extent) on quality of life variables. This was explained by positive expectancy effects of the 
drug and care. In this study, 9 patients receiving placebo $(8 \%)$ and 5 receiving transdermal estradiol (4.5\%) noted uterine bleeding during the study. Withdrawal bleeding was inversely related to the number of months since the last menstrual bleeding. (Wicklund, Karlberg, $\&$ Mattson, 1993). Another study showed that on all assessments of the quallity of life in 499 postmenopausal women, HRT was superior to symptomatic treatment for flushes (verapipride). Important is that this effect was independent of the incidence of hot flushes, since it has been suggested that the flushes have a negative impact on the quality of sleep, in that sleep is disturbed by the incidence of nightly flushes and perspiration, which in turn might negatively affect cognitive function and well being (Lmouzin-Lamothe, Mairon, Joyce, \& Le Gal, 1994).

Wable 4: Experimental sudies into the effect of HRT on cogniti we functions in normal elderly women

\begin{tabular}{|c|c|c|c|c|c|}
\hline Authors (year) & Subjects (age) & Design & ERT & Test & Result \\
\hline $\begin{array}{l}\text { Camplyell ef all } \\
1977\end{array}$ & $\begin{array}{l}\text { linic, } n=61 \\
(50-79 \mathrm{yrs})\end{array}$ & $\begin{array}{l}\text { crossov } \\
6 \text { minth }\end{array}$ & $\begin{array}{l}\text { CEREI } \\
1.25\end{array}$ & $\begin{array}{l}\text { Quality of Life } \\
\text { subij. memory }\end{array}$ & $\neq$ \\
\hline $\begin{array}{l}\text { Rauramo } \\
1975\end{array}$ & $\begin{array}{l}B S O p t_{i} n=88 \\
48.9(30-55)\end{array}$ & $\begin{array}{l}\text { between } \\
6 \text { minh }\end{array}$ & $\begin{array}{l}\text { estrad } \\
2 \mathrm{mg}^{2}\end{array}$ & $\begin{array}{l}\text { Subj mood/sasomotor } \\
\text { Memory, RT, Logical }\end{array}$ & $\begin{array}{l}\neq 1 / 7 \\
\mathrm{~ns}\end{array}$ \\
\hline $\begin{array}{l}\text { Wanhulle et al } \\
1976\end{array}$ & $\begin{array}{l}\text { nums. } n=26 \\
57.6\end{array}$ & $\begin{array}{l}\text { between } \\
3 \text { minth }\end{array}$ & $\begin{array}{l}\text { estriol } \\
4 \mathrm{mg}\end{array}$ & $\begin{array}{l}\text { Alertness, Attention } \\
\text { Memory (span), Conc }\end{array}$ & $\begin{array}{l}\neq \\
n s\end{array}$ \\
\hline $\begin{array}{l}\text { Fedorfreyberg } \\
1977\end{array}$ & $\begin{array}{l}\text { patients, } n=21 \\
56.5(47-70)\end{array}$ & $\begin{array}{l}\text { between } \\
3 \text { minth }\end{array}$ & $\begin{array}{l}\text { estrad } \\
2 \mathrm{mg}\end{array}$ & $\begin{array}{l}\text { Autention (Stroop, STM } \\
\text { CRRT, Conc, HDS, EPI }\end{array}$ & $\begin{array}{l}\neq \text { (base } \\
+ \text { con) }\end{array}$ \\
\hline $\begin{array}{l}\text { Hatkman ex al } \\
1977 \text {. }\end{array}$ & $\begin{array}{l}\text { patients, nz: } 18 \\
(29-68 \text { yrs })\end{array}$ & $\begin{array}{l}\text { between } \\
6 \text { mnth }\end{array}$ & $\begin{array}{l}\text { estro } \\
1.5 \mathrm{mg}\end{array}$ & $\begin{array}{l}\text { Subj/vasomotor } \\
\text { Memory }\end{array}$ & $\begin{array}{l}\neq \\
\mathrm{ns}\end{array}$ \\
\hline $\begin{array}{l}\text { Sherwing all } \\
1988\end{array}$ & $\begin{array}{l}\text { BSO pt. } n=50 \\
454 \text { yrs }\end{array}$ & $\begin{array}{l}\text { crossov } \\
3 \text { month }\end{array}$ & $\begin{array}{l}\text { estrad } \\
10 \text { magn }\end{array}$ & $\begin{array}{l}\text { Memory (spantrecall) } \\
\text { Reasoning, speed }\end{array}$ & $\neq$ \\
\hline $\begin{array}{l}\text { Sherwin at al } \\
1990\end{array}$ & $\begin{array}{l}\text { BSO pi, } n=12 \\
47 \text { yrs }\end{array}$ & $\begin{array}{l}\text { between } \\
2 \text { mnth }\end{array}$ & $\begin{array}{l}\text { estrad } \\
10 \mathrm{mgnn}\end{array}$ & $\begin{array}{l}\text { Memory(paragraph, } \\
\text { pared), Visuall memory }\end{array}$ & $\begin{array}{l}\neq \\
\mathrm{ns}\end{array}$ \\
\hline $\begin{array}{l}\text { Phillpis et all } \\
1992\end{array}$ & $\begin{array}{l}\text { BSO pts, } n=19 \\
48.2 \pm 4.7\end{array}$ & $\begin{array}{l}\text { between } \\
2 \text { mnth }\end{array}$ & $\begin{array}{l}\text { estrad } \\
10 \mathrm{mgnn}\end{array}$ & $\begin{array}{l}\text { Moodimenopausal sym } \\
\text { Memary }\end{array}$ & $\begin{array}{l}\mathrm{ns} \\
\neq\end{array}$ \\
\hline $\begin{array}{l}\text { Dilkoff of al } \\
1991\end{array}$ & $\begin{array}{l}\text { 7. A. } n=36 \\
73(45-60)\end{array}$ & $\begin{array}{l}\text { between } \\
3 \text { moth }\end{array}$ & $\begin{array}{l}\text { CEE } \\
0.625\end{array}$ & $\begin{array}{l}\text { Quality of life (Dep) } \\
\text { Memory (spani),DSST }\end{array}$ & $\begin{array}{l}\neq \\
\text { ans }\end{array}$ \\
\hline $\begin{array}{l}\text { Wiklund on al. } \\
1993\end{array}$ & $\begin{array}{l}\text { volunt } n-22 \pi \\
53 \pm 4(45-65)\end{array}$ & $\begin{array}{l}\text { between } \\
3 \text { month }\end{array}$ & $\begin{array}{l}\text { estra } \\
50 \mu g\end{array}$ & Quatily of life & $\neq$ \\
\hline $\begin{array}{l}\text { Lebmonzin at at. } \\
1994\end{array}$ & $\begin{array}{l}\text { phys: } n=479 \\
51 \pm 2.52\end{array}$ & $\begin{array}{l}\text { between } \\
6 \text { mith }\end{array}$ & $\begin{array}{l}\text { estrad } \\
0.05 \mathrm{mg}\end{array}$ & Quality of life & $\neq$ \\
\hline
\end{tabular}

1) conjugnted esurogens (CEE) ; 2) cstradiol valerate or valerianate; 3) piperazine oestrone sulphate $2 x /$ day

4) estradiol transderrnall (P added); BSO: bilateral salpingo oophorectomy; TAH: total abdominal hysterectomy

However, in a double blind study with 36 asymptomatic (without hot flushes), surgically postmenopatusal women aged 45-60 yrs, only the income management scale of the Profile of Adaptation to Life was seen to improve with CEE ( 0.625 and $1.25 \mathrm{mg}$, no dose effects), while negative emotions, well being, and physical symptoms were not different between groups. The Beck Depression Inventory was significantly different, but only between pre- and posttreatment scores. Furthermore, in this study no effects of ERT were found on the WAIS Digit Span and the Digit Symbol Test, tests for STM and attention. Also, no effect was seen on the Minnesota Multiple Personality Inventory (MMPI), although this may be expected, since personality is a trait characteristic which is not easily influenced by therapy 
It is thus unclear whether the effects of HRT reported on cognition are mediated by or are independent of changes in mood and general well being (Backstrom, Bixo, Hammarbick, 1985). For instance, Klaiber, Broverman, Vogel, \& Kobayasthi (1979) fownd that estrogens as compared to placebo decreased symptoms in severly depressed women. Yet, the dosage used in this study was very high (15-25 mg of oral conjugated estrogens) (Klaiber, Broverman, Vogel 8 Kobayshi, 1979, also see: Klaiber, 1982) Schneider, Brotherton, \& Hailles (1977) found that at much lower dose of conjugated estrogens $(0.3 \mathrm{mg})$ over a four week period was able to improve mood as measured with the Beck Depression Inventory in non depressed women, but not in depressed postmenopausal subjects. Six out of 10 depressed subjects even got worse. The depressed women did, however, report more psychological and sociosituational stress and no statistical evaluation of results was given in this study (Schneider, Brotherton, \& Hailes, 1977). Yet, other studies have also not found promising results of ERT in alleviating mood in depressed patients (Ottowitz \& Halbreich, 1995). Furthermore, some researchers (Philips and Sherwin, $1992 \mathrm{~b}$ ) state that the effect of estrogens is independent of the effect of mood, since in their study verbal memory was enhanced in the absence of an improvement in mood as measured with the Multiple Affect Adjective Check List (MAACL). This list is stated to be a valid and reliable measure of affect changes (anxiety, depression, or hostility) and concurrent hormone levels (Phillips \& Sherwin, 1992). However, since many other investigators did find effects of ERT on an improvement of mood in non depressed postmenopausal women, it is possible that the sensitivity of this questionnaire is too low.

Sherwin (1994) claimed that there is sufficient evidence to support the contention that estrogens influence cognitive functioning in women and that this is a specific and not a global cognition enhancing effect, e.g. on verbal memory, articulation and fine motor skills, but not on visual/spatial memory or attention (Kampen \& Sherwin, 1994; Sherwin, 1994). For instance, in one of her earlier studies (Sherwin, 1988), on average 10 words more with ERT were recalled on the paragraph recall test as compared to placebo. Also, Sherwin and Philips (1990) found that in a between groups study, the surgically postmenopausall women on ERT had higher scorres on the immediate and delayed paragraph recall as compared to placebo users (on average 21 as compared to 18). On the Paired-Associate test, the scores in the placelbo group showed a decline 2 months postoperatively. Visual recall showed no effects of ERT. The graphs, however, reveatled large standard errors on the paragraph recall score. Philips and Sherwin (1992) found effects of ERT $(n=10)$ as compared to placebo $(n=9)$ on the immediate paragraph recall, and the immediate and delayed recall of Paired-Associates. No differences were found on visual reproduction memory, digit span or delayed paragraph recall. Also, no difference was found with respect to sleep difficulties, subjective concentration or other (psycho)somatic subscales of the menopausal index or in anxiety, depression, or hostility as measured with the Multiple Affect Adjective Checklist (MAACL). However, a closer look at the data in this study reveal nonsignificant preoperative differences, which may have confounded effects postoperative and which make clear cut conclusions difficult. Also, on Paired-Associate learning test, the effect was caused by deterioration of the placebo group and not by improvement of the HRT groups.

In contrast, Vanhulle and Demol (1976) found no effects on memory (as measured with the Digit span) but did find effects on alertness (visual sensitivity on a vigilance task) and attention (Bourdon-Wiersma test) in a parallel groups study with nuns. Fedor-Freyberg (1977) also found non specific improvements after 3 months of HRT $(n=11)$ on the performance of the Choice RT, the Stroop test, the Konzentrations Verlauf Test and an attention test measuring short term memory and reasoning ability as compared to placebo $(n=10)$. Also, the Eysenck Personality 
Inventory (EPI) showed an increase in extraversion in ERT users, similar to the scores on several general symptoms (sleep disturbiances, fatigue, anxiety, depression, irritability). Again, Goebel, Birge, Price, Hansion, \& Fishel (1995) concluded that an 8 month double-blind HRT therapy in 87 elderly females (age $>69$ ) did not affect central processing speed (as measured with the Trial Making "Test B) or postural stability relative to placebo controls. The Trail Making Test B measures similar processes as compared to the Stroop. These tasks are very susceptible to age effects (Houx, Vreeling \& Jolles, 1991) and hence the age of the subjects in the latter study may have been an intervening variable.

\subsection{Discussion and methodological considerations}

The data of the population based studies suggest that effects of HRT is either very specific (function or age-group) or abisent. The study by Barret-Connor et al (1993), for instance, revealed no effects. The study by Kampen and Sherwin (1994) only revealled effects on one of many tests used and in addition, these effects were wery small, which is suggestive of chance findings. The findings of Robinson et al (1994) underlie this notion. Furthermore, it is difficult to compare these studies to the study by Kimura (1995) because she used different statistical methods to analyze results.

In the experimental studies, methodological differences may explain part of the contrasting results found. First in most studies, power analyses determining the necessary number of subjects needed to show significant effects are not presented. Second, the design that is employed in a study may result in a different outcome. For instance, crossover designs are very sensitive to change. A crossover study by Sherwin (1988) revealed that the number of digits temporarily stored in short term memory (Digit Span) significantly increased with 3 digit (from 6.4 to 6.7) with the use of estrogens. Parallel studies need much larger differences due to a larger variance in interindividual performance to show the effect of a drug. Third, some of the tests used are known not to be very sensitive to change (e.g. Raven Progressive Matrices: Rauramo, 1975). For instance, the lack of findings of estrogens on attentional functions as stated by Sherwin (1994) could be attributed to the use of insensitive tests. Namely, estrogens have been found to affect perceptual speed and recognition sensitivity, which can be seen as indicators of an increase in arousal (Sanders, 1986). Also, choice RT (an indicator of enhanced activation) was better in postmenopausal women that used estradial valerate $(n=11)$ as compared to placebo $(n=10)$ (Fedor Freyberg, 1977). "These findings may be suggestive of global effects of ERT on cognitive functions.

Fourth, most studies have not measured actual circulating levels of estrogens (Sherwin, 1994). Actual compliance hence is not known. This can be a problem, especially since dropouts due to side effects (rashes, pains, menstrual bleeding) have been seen to occur during the course of these studies. Fifth, although double blind procedures are essential in (cognitive) drug evaluation studies, this is difficult in natural postmenopausal HRT research. Many women show at least some spotting at the end of the cycle due to withdrawal bleeding necessary to prevent endometrial proliferation. Also, vasomotor and other complaints are usually quickly eliminated by HRT. It is thus difficult to conduct these studies double-blind. Hence, since women are familiar with the experimenters hypotheses, the expectancy of positive HRT effects may have confounded results. On the other hand, studies performed by Sherwin $(1988 ; 1990 ; 1991 ; 1992)$ were able to show double blindly that surgically menopausal women, who had undergone hysterectomy and hence showed no withdrawal. bleeding, had positive effects of estrogens on memory functions. Again, these women were in general younger than the postmenopausal women receiving HRT and interactions between age and HRT in the strength or quality of effects may have occurred. A drug 
like Tibolon could be a good alternative in postmenopausal women, since no withdrawal bleeding occurs. Although Tibolon shows positive effects on osteoporosis, in some systems it is mentioned to have antioestrogenic and progestogenic effects. So far effects of Tibolon on cognitive functions or cerebral effects are unclear.

Sixth, the different HRT preparations and differences in the time of measurement may also account for some of the differences in results found. In traditional HRT, the addition of a progestagen in the last phase of the cycle and the induced withdrawal bleeding is thought necessary to reduce the effect of estrogens on endometrial proliferation, which may result in endometrial cancer (Campbell \& Whitehead, 1977; Judd, 1988). As early as 1954, Merryman et al found that progestagens may induce sleepiness (Merryman, Boidman, Barnes " Rothchild, 1954). In addition, Bäckström et al. (1985) mentioned that progestagens decrease brain excitability, which is indicative of their sedative effects. Furthermore, they reported that postmenopausal women $(n=22$ ) that had gestagen (a progestagen) added in the last week of the cycle showed significant more cyclicity in their mood and more physical signs (e.g. breast tenderness) as compared to the women only using estradiol (for 3 weeks) (Bäckström, Bixo ${ }_{i}$ \& Hammarbäck, 1985). Also, Sherwin (1991) showed that the combination of conjugated estrogens and $5 \mathrm{mg}$ of medroxyprogesterone acetate induced more negative moods and a greater psychological symptomatology as compared to equivalent monotherapentic dosages of estrogens. Other studies showed similar effects with ethinyl estradiol combined with levonorgesterel (Dennerstein, Burrows, Hyman, \& Sharpe, 1979) and estradiol combined with norethisterone (Magos et al., 1986) as compared to estrogen therapy alone.

\section{Possible mechanisms of action}

\subsection{Introduction}

There are several plausible mechanisms through which estrogens may exert their protective and enhancing effects as have been found in vitro and in vivo experimental animal and human studies. Direct effects on cognitive functions in aging and dementia can be explained on a functional level, i.e. in enhancing neurotransmitter functions which may be implicated in cognitive aging and dementia. Also, estrogens may affect the hardware of the brain indirect through their protective actions upon the vascular system, and direct, through their actions at it neuronal level, in affecting spine outgrowth and in the protection of neurons against oxidative stress induced cell death. The current section discusses actions of estrogens and possible opposing actions of progestagens in more detail.

\subsection{Neurotransmittersystem actions}

\section{- The monoaminergic system}

Monamines, such as norepinephrine (NE) (McEntee \& Crook 1990$)$, serotonin (5-HT) (McEntec \& Crook, 1991), and dopamine (DA) (Ollat, 1992) have shown decreased activity in aging and dementia. Brainstem catecholaminergic (NE and DA) neurons contain small. numbers of estrogen receptors, which suggest that it is possible that ERT can exert effects through enhancement of catecholaminergic function (McEwen, Alwes, Bulloch, \& Weiland, 1997). Estradiol may enhance the excitability of noradrenergic netrons by potentiating the excitatory effect of alphal adrenergic receptors. Progestagens were found to depress norepinephrine-induced $c A M P$ formation in estrogen-primed rats (Petitti \& Etgen, 1990). Some investigators found that estradiol increases the noradrenergic turnover in rat brain by acting directly upon adrenergic (alphal) 
receptors (Hiemke, Banger, Kohsik, Hundt, \& Ghraf, 1992). Also, estrogen was seen to induce an increase in tyrosine hydroxylase activity, which results in an increase in $\mathbb{N E}$ synthesis (Beattie, Rogers, Soyka, 1973). However, this was not found by others (Luine McEwen, 1977). Progestagens have been seen to reverse the effect of estrogens on tyrosine hydroxylase (Beattie ef al. 1973). Furthermore, an increase in NE activity can be due to decreased NE reuptake. In this line, estradiol was found to inhibit the uptake of norepinephrine by synaptosomes (Janowsky \& Daris, 1970). Also, increased NE activity may be due to the inhibition of monoamine oxidase (MAO) activity (Luine, 1975; Luine \& McEwen, 1977). MAO degrades the activity of monoaminergic neurotransmitters. Hence, if MAO is inhibited, more monoaminergic neurotransmitters become available. MAO has been found to be less actiwe when estrogen levels. are high, e.g. in the preovulatory phase of the menstrual cycle (Klaiber et al., 1979). A 30\% decrease in the activity of type $\mathrm{A}$ MAO (involved in the degradation of NE and 5-HT) in basomedial-hypothalamus and corticomedial-amygdala was induced by the administration of estradiol, probably due to enhanced degradation of MAO-A (Luine et al., 1975; Luine \& McEwen, 1977). Medroxyprogestrone has been reported to reverse the estrogen-induced inhibition of MAO-activity in women (Klaiber, Kobayashi, Broverman, \& Hall, 1971).

Estrogens have been found to increase the serotonergic postsynaptic (5-HT1A) sensitivity and increase both the number of serotonin $(5-\mathrm{HT})$ receptors and 5 -HT reuptake. Estrogens also increase 5-HT synthesis (Halbreich, 1997). It has been shown that untreated post-menopausal women have blunted neurohormonal responses (i.e. cortisol and prolactin) to m-CPP challenge, when compared to reproductive controls. ERT (estradiol) partially restored neurohormonal responses to m-CPP treatment. The findings can be considered a demonstration in vivo of the dysregulation of the serotonergic system in postmenopausal women and the restorative influence of ERT on the serotonergic system (Halbreich et al., 1995b). Furthermore, estrogens have been reported to increase the 5-HT binding sites on platelets which enhances the transport of serotonin (Sherwin \& Suranyi Cadotte, 1990). Lastly, estrogens can regulate the amount of free tryptophan available to the brain (Aylward, 1973), which is metabolized to serotonin. Progestrone may reduce the estrogen-induced serotonergic activity through reversal of the decreased MAO-A activity (Holzbauer \& Yondin, 1973). However, in ovariectomized rats, treatments with estrogen, progestrone and the combination of both were seen to positively affect the serotonergic system (McEwen et al., 1997). In contrast, some investigators found a paradoxical fall of NE and 5-HT concentrations in the brain stem-hypothalamus area in ovariectomized rats treated with estrogen. However, a rise in 5-HT and NE was seen when ovariectomized rats were treated with estrogen followed by progestrone. Also, the level of gamma amino butyric acid (GABA) was seen to decrease with estrogen use. The addition of a progestagen was seen to elevate GABA above nomal values, which can explain its sedative and tranquillizing effects (Das \& Chaudhuri, 1995). Similarly, others suggested that progestagens may reverse the excitatory effects of estrogens through their CNS depressant actions (Bäckström et al., 1985).

As for the effects on dopaminergic systems, estrogens may modulate some aspects of the function of the dopaminergic systems of the forebrain (see: VanHartesveld \& Joyce, 1986 for a review). Estrogens are thougtht to have a facilitating, probably normalizing action on mesolimbic dopanine. Furthermore, clinical studies suggest that estrogen negatively affects extrapyramidal disorders through a suppression of striatal dopaminergic function. However, the direction of the effect has not been consistent. The effect may be dose-dependent, e.g in the mesostriatal complex high doses of estrogen suppresses the dopaminergic functions. However, this initial suppression is reversed by catecholestrogen (a metabolite of estrogen) and progrestrone. A low dose of estrogen does not show an altered enhancement of dopaminergic striatal functions (Hartesveldt van \& 
Joyce, 1986 ).

The cholinergic system

Acetylcholine has long been suspected to play an important role in memory and other comnitive dysfunction in aging and dementia (Bartus, Dean III, Beer, \& Lippa, 1982). In AD patients, there is a marked decrease in the activity of choline acetyltransferase (CAT) in the cerebral cortex and hippocampus (Coyle, Price, \& DeLong, 1983). CAT is an enzyme that is involved in the synthesis of acetylcholine. Estradiol has been found to increase the activity of CAT most prominently in the rat basal forebrain, but also in the amygdala and preoptic area (Luine, Park, Joh, Reis, \& McEwen, 1980; Luine, 1975; Luine \& McEwen, 1983; Simpkins et al., 1994). Changes im enzyme activity were positively related to the dose of estradiol administered and were blocked by the estrogen antagonist MER-25 (Luine et al., 1975). Simpkins at al. found that little effect of hormonal environment (ovariectomy with or without 17-beta-estradiol) on CAT occurred after 5 weeks, suggesting that little or no loss of innervation occurred. However, after 28 weeks, the CAT level had fallen to $56 \%$ in the frontal cortex of ovariectomized controls, but by onlly $16 \%$ in the ERT rats. In the hippocampus there was a similar, albeit smaller, preservation of CAT activity with ERT. Also the data by Luine et al (1980) suggest estradiol administration resulted in small and consistent increases in CAT activity in the preoptic after chronic (after 24 and 72 hours) but not acute (after 3 hours) treatment.

\subsection{Neuronal protection}

\section{- Nerve growth factor actions}

Simpkins et al (1994) hypothesized that estrogens may preserve the integrity of basal forebrain cholinergic neurons. Estrogens have, for instance, been reported by several authors to act on nerve growth factor (NGF)-related systems and basal forebrain cholinergic neurons (e.g. Gibbs, 1994). Illustrative is the work of (Toran Allerand et al., 1992) who demonstrated that estrogen receptors are co-localized with low affinity nerve growth factor receptors. As such, estrogens may influence neuronal survival, differentiation, regeneration and plasticity. Estrogen-neurotropin interactions may be important for the survival of cholinergic neurons and may underlie the protective effects of HRT against the development of Alzheimers disease. Indeed, other authors also found stimulating effects of estrogens on neuronal regeneration and modulatory actions of estrogens upon long and short term synaptic function (Wong \& Moss, 1992). Again, continuous estrogen treatment was seen to result in a down regulation of NGF and NGF receptors, which may counteract the initial beneficial effects on basal forebrain cholinergic functions(Gibbs, 1994). Yel, the author hypothesized that the combination therapy of estrogens and progestagens may help maintain the positive effects on cholinergic systems.

Furthermore, the hippocampus is a structure important in learning and memory and has been seen to contain estrogen receptors (Pfaff, 1980). Owariectomy in female rats was reported to decrease the spine density in the CA1 region of the hippocampus, which does not occur with estradiol treatment (Gould, Woolly. Frankfurt, \& McEwen, 1990). These changes can take place within 24 hours. For instance, during the female rat oestrous cycle, there is a rapid cyclic fluctuation in the spine density in the CAl region of the hippocampus. Estrogens were seen to provake an increase in dendritic spine density. Progesterone rapidly potentiated the estrogen-induced spine formation, but then triggered a down-regulation of spines on CAI neurons. When estrogen was withdrawn, the down-regulation of spines occurred slowly, but when progesterone was administered, it took place within 8-12 hours. Furthermore, a progesterone antagonist (RU486) blacked the natural down-regulation of dendritic spines between the 
pro-oestrus peak and the through on the day of oestrus. When both steroids were at their loweat levels, spine density was also lowest (Woolley \& McEwen, 1993; Woolley, Gould, Frankfurt, \& McEwen, 1990). Hence, it is possible that steroids can modulate the excitatory input to CAI pyramidal neurons through a regulation in the number and/or organization of excitatory synapses and may be inwolved in memory formation. Lastly, estrogens have been seen to induce an increase in muscarinic (cholinergic) receptors in the hypothalamus (Janowski \& Rausch, 1985)

\section{- Cerebrovascular and antioxidant effects}

Prophylactic effects of estrogen in normal aging but also in vascular or multiple-infarct dementia may be explained through their anti-atherosclerotic and vasodilator effects. A decreased cerebral blood flow due to atheroselerotic effects has long been suspected to be an important factor in agemelated decline and dementia (Smith, 1984). Postmenopausal women had, after adjustments for age and oher indicators of candiowascular risk, a 3.4 times greater risk of atherosclerosis than premenopatisal women (Witteman, Grobbee, Kok, Hofman, \& Valkenburg, 1989). (Cerebro) vascular disease, such as atherosclerosis $s_{*}$ can lead to hypoxia and eventually to ischemic insults, resulting in multiple infarcts and cell death in the brain. Extensive neuronal loss is seen in the CAl region of the hippocimpus, basal forebrain and association neocortex has been found to occur in Alzheimer's Disease (Coyle et al., 1983).

Estrogens have been found to protect against the development of atherosclerosis. Indirect, through their actions on plasma lipids and plasma lipoproteins and direct through the inhibition of the formation of atherosclerotic plaques and other vascular effects, such as the estrogen induced marked vasodilatation in systemic and coronary arteries (Godsland, Wynn, Path, Crook, \& Miller, 1987; Judd, 1987; Riedel, Rafflenbeul, \& Lichtlen, 1993; Sarrel, 1989; Sarrel, 1990; Stampfer \& Colditz, 1991). The effects found in the study by Funk, Mortel, \& Meyer (1991) are in concordance with the arterial mechanism mentioned, in that a trend for increased cerebral perfusion was seen in HRT users. Also, Burns and Murphy (1996) found that estrogens improve cerebral blood flow, through reversal of the diminushed vessel wall compliance associated with the menopause. Estrogens produced an increase in cerebral perfusion associated with improved cognitive function. However, many of the positive effects that estrogens may exert on the cerebrowascular system are reversed by the addition of progestagens, in that a decrease in cerebral blood flow and a reversal of the anti-atherosclerotic effects has been described (Sarrel et al, 1989). However, medroxyprogestrone (as compared to noresthisterone), natural progestrone and third generation progestrone (desogestrel) have been reported not to interfere with the estrogen mediating improvenents in serum lipid levels (Ottowitz \& Halbreich, 1995)

- ApoE and antioxidant activity

AD is characterized pathologically by the accumulation of intracellular neurofibrillary tangles and senile plaques. These plaques and tangles show the presence of B-amyloid formation. Estrogens may affect the production of amyloid precursor protein (APP) and could decrease cerebral amyloid deposition in AD. For instance, treatment with estradiol is associated with accumulation of the soluble amino-terminal cleavage product of APP, which is indicative of nonamyloidogenic processing. There were no changes in levels of intracellular immature or mature APP holoproteins, which suggests that estrogen may increase the secretory metabolism of APP and reduces the accumulation of the neurotoxic B-amyloid fragment of APP (Jaffe, ToranAllerand, Greengard, \& Gandy, 1994). Apolipoprotein E (ApoE) accelerates precipation of B-protein. ApoE has been found to be suppressed with HRT (Honjo et al., 1995).

The amyloid B peptide can directly damage neurons and increase their vulnerability to excitotoxic and metabolic insults, through the induction of free radicals (Mattson et all., 1993) However, estrogens may have an inherent antioxidant activity, as data from studies of mechanisms 
of oxidative injury to hippocampal cells show (Goodman, Bruce, Cheng, \& Mattson, 1996). It was found that both estrogens and progesterone (to a lesser extent) can protect cultured hippocampul meurons against glutamate toxicity, glucose deprivation, FeSO 4 taxicity, and amyloid B-peptide toxicity. These factors may contribute to neuronal degeneration in various age-associated disorders, such as atherosclerosis and Alzheimer's disease (Goodman et al, 1996).

\subsection{Discussion}

In sum, neurotransmitter systems that may be implicated in cognitive aging and dementia and that have been found to be positively influenced by estrogens are the cholinergic and the monoaminergic neurotransmittersystems. Estrogens have been reported to increase levels of neurotransmitters, through their influence upon the availability of precursors, upon the activity of enzymes needed for synthesis and degradation of neurotransmitters. Estrogens have also been reported to act upon receptors and may even affect the structural hardware of the brain, which can explain their protective effect, especially in Alzheimer"s disease and in the long term effects of aging. Most effects of estrogens are opposed by progestagens, such as effects on the noradrenergic systems (Beattie et al., 1973; Klaiber et al., 1971) the dendritic outgrowth (Woolley \& McEwen, 1993; Woolley et al., 1990), and potential cerebrovascular effects (Sarrel. 1990). Cholinergic, serotonergic, and the potentially protective (i.e. antioxidant) actions of estrogens are probably unopposed by progestagens.

\section{General discussion}

Research into cognition in aging and dementia has a common problem, namely the profound heterogeneity within groups of subjects, in terms of education, morbidity, and the large variation in the severity of cognitive decline (Riedel \& Jolles, 1996). However, overall, performance. decrement in intellectual functions, memory, perception, behavioral organization, and motor functions have been noted. Elderly subjects, however, are relatively less impaired in tasks in which they can rely on well-established skills and knowledge. Although an overall slowing of cognitive functions is seen (Poon, 1993), subjects are especially slowed on complex tasks under the pressure of time. With respect to the early phases of $\mathrm{AD}$, the pattern of cognitive deficits seems similar to that seen in normal aging. In addition, the dementing subject is characterixed by deficiencies in verbal functions and recognition memory. The elder subjects seem to lose the ability to retrieve information from long term memory. A consolidation deficit appears in the early phases of dementia (Jolles, 1986). Presently, much research effort is devoted to establishing psychometric differences between the so called "normal aging' and early stages of dementia (Jolles, Houx, Van Boxtel, \& Ponds, 1995; Jolles, Verhey, Riedel, \& Houx, 1995b).

The present review has shown that it is plausible that estrogens have protective and enhancing effects on cognitive functions in aging and mild to moderate dementia and may exert their protective and enhancing effects though acting upon many biological levels. However, evidence for protective effects in the normal population is too inadequate to call for a general hormonal substitution therapy for all women. Possible, women genetically at risk in developing Alzheimer"s dementia, should be advised of the protective effects of estrogens. Risk for the development of breast cancer should be taken into account, in that women with family history of breast cancer should be contraindicated and others should be carefully screened (Lichtman, 1996). The risk for endometrial cancer is reduced with the addition of progestagens (e.g. Campbell et al, 1977).

The literature describes that progestagens may reduce the positive effect of estrogen when both 
are given together. Many of the effects that estrogens exert on the brain are reversed by the addition of progestagens, such as the positive effect on noradrenergic neurotransmitter systems, dendritic spine outgrowth, and vascular functions. Yet, the effect of the combined therapy of estrogens and progestagens on cholinergic and serotonergic systems and the protective effect on neuronal injury are probably maintained. Chollinergic systems are thought to be involved in memory dysfunction in aging and dementia (Bartus et al, 1982). The maintenance of cholinergic systems may thus be a crucial factor in healthy aging. Serotonin has been found to be decreased especially in dementia. Since serotonin has been found to positiwely affect memory functions, possibly through its effect upon the cholinergic system (McEntee \& Crook, 1991), its actions may be adjuvant in preserwing memory functions. In this line, for instance, no negative effect of the addition of a progestagen was seen on memory functions (Kampen \& Sherwin, 1994).

It was mentioned that some authors (e.g. Sherwin, 1994; 1996) believe that the effect of estrogens is specifically on memory funetions and is independent of mood. Several studies found that the addition of progestagens reversed the positive effects of estrogens on mood. However, other studies suggest (e.g. Limouzin-Lamothe, 1994) that general well being is not negatively affected by the combined therapy. Furthermore, some of the earlier studies (e.g. Caldwell, 1952) found that the positive effect of ERT plateaud or decreased after one year.

It should thus be investigated whether long term combination therapy positively affects memory, and speed of information processing, but also arousal, subjectively experienced activation, mood and other indicators of well-being. Also, the inability to test double blind in normal postmenopausal women due to withdrawal bleeding and the confounding influence of the expectancy of positive results should be investigated in a normal population sample of women in the late middle-age and early senium. The subjects should be unaware of the actual purpose of the study. Also, more controlled studies into the long term protectiwe effects of estrogens: in elderly women in an early stage of Alzheimer's disease should be undertaken. A combination of psychometric tests with techniques based upon information processing paradigms and behavioral neurology will prove to be a fruitful approach to establisth relations between behavioral and cognitive functions and the underlying cerebral substrate (Jolles, 1986; Jolles et al, 1995b). Specific dementia sensitive cognitive batteries, including verbal memory tasks demanding both retrieval and recognition to test the effects on consolidation functions and tests which measure the speed of information prosessing, should be used to optimally detect cognitive improvement. 


\title{
Chapter 11
}

\section{Hormonal Replacement Therapy and cognitive performance in middle-aged women: results from a large cross sectional study}

E. Hogervorst, W.J. Riedel, M. P. J. wan Boxtel, \& J. Jolles

\begin{abstract}
Estrogen Replacement Therapy (ERT) in postmenopausal women could be a protective factor against Age-Related Cognitive Decline (ARCD) and Alzheimer's Disease (AD). The rationale of the present study was to evaluate whether ERT-use is associated, in a positive manner, with cognitive functioning in middle-aged women. The study was performed as a separate analysis within the Maastricht Aging Study (MAAS), where 1869 subjects aged 24 through 82 were inwestigated both medically and neuropsychologically. Twenty-three ERT-users were found in the age range of 45-65 years. A case-control design was used where these subjects were compared to 319 controls from the same age range. Statistical analyses were performed to assess differences between estrogen-users and controls on socio-demographic-, health risk-, perceived health- and cognitive function variables. Furthermore, the independent and the possible confounding influence of age, education, health risk factors- and perceived health varialoles on ERT-use and its association with cognitive performance respectively, were determined.

Unexpectedly, ERT users had more subjective complaints of memory dysfunction as well as of anxiety and depression. No effects of ERT were found on objective memory performance or cognitive flexibility. Although ERT-users had more years of education, a trend towards a positive relationship between ERT-use and sensorimotor speed score of objectively measured cognitive performance was detected, which was independent of the effect of age and education. The small proportion of ERT-use in the MAAS database is probably representative for the Netherlands. Furthermore, it should be kept in mind that about half of these women had undergone hysterectomy and/or ovariectomy, which may explain why this group overall had more psychological complaints. As far as the explorative nature of the analysis permits, the results showed a trend in the direction of a general activating effect of ERT-use, rather than a specific association between ERT-use and memory or cognitive performance.
\end{abstract}

\section{Introduction}

Some neurons or groups of neurons are functionally dependent on estrogen levels (Fillit, 1994). Following this premise, low estrogen levels may lead to a decline in neuronal plasticity and structural neuronal loss. Estrogen receptors have been found to be co-Jocalized with low affinity nerve growth factor receptors and through this pathway may influence neuronal survival, differentiation, regeneration and plasticity. Estrogen-neurotropin interactions may be important for the survival of cholinergic neurons and through this mechanism may protect against the 
development of Alzheimer's disease (Toran Allerand et al., 1992). It has therefore been suggested that Estrogen Replacement Therapy (ERT) in postmenopausal women could be a protective factor against Age-Related Cognifive Decline (ARCD) and Alzheimer's Disease (AD) (Halbreich et al., 1995).

Severil authors have suggested that ERT could reduce the risik of AD and Vascular Dementia (VD) or Multi-Infarct Dementia (MID) (Funk, Mortel, \& Meyer, 1991; Lopes, Mensier, Laurent, \& Besse, 1995; Simpkins, singh, \& Bishop, 1994). A recent study of 1124 elderly women (aged mean 74.2 (s: 7.0 ) years), showed that $5.8 \%$ of ERT users were suffering from Alzheimer's Disease, as compared to $16.3 \%$ of non users. A greater reduction in risk was seen when ERT was taken for a longer period of time, even when adjusted for education, ethnic origin, and apolipoprotein- $\mathrm{E}$ genotype (Tang et al, 1996). In a case-control study $11 \%$ of a group of female patients diagnosed as suffering from 'probable $A D$ ' had received estrogen replacement therapy as compared to $20 \%$ of a matched control group (Mortel \& Meyer, 1994). The authors concluded that the risk of developing dementia without estrogen replacement therapy hence seemed doubled. In another case-control study $7 \%$ of 143 female patients diagnosed as suffering from 'probable Alzheimer Disease' had received ERT, as compared to $18 \%$ of 92 non-demented contral subjects (Henderson, Paganini Hill, Emanuel, Dunn, \& Buckwalter, 1994). Beneficial effects of ERT on cognitive functioning in female patients diagnosed as suffering from "probable AD' have also been found in several clinical studies (Fillit et al., 1986; Honjo et al., 1989; Ohkura et al., 1995).

Although these findings suggest a possible protective effect of estrogens as to the development of age-related cognitiwe decline and dementia in elderly women, several studies did not find protective effects of ERT use and Alzheimer's disease (Amaducci et al., 1986; Bremmer et al., 1994; Broe et al., 1990). Furthermore, case-control studies only found marginal effects of ERT use on cognitive functions in normal postmenopausal women (Kampen \& Sherwin, 1994; Robinson, Friedman, Marcus, Tinklenberg, \& Yesavage, 1994). In a prospective study (Barrett Connor \& Kritz Silverstein, 1993) no protective effect of ERT use was detected in preventing cognitive decline. In this study, 800 women (aged 65-95 years) were investigated. Estrogen use was evaluated at baseline and at a follow-up 16 years later. Almost half of the cohort had used estrogen at some time after the menopause and one third were current users. Age-related cognitive decline was similar for all women even when adjusted for age and education. Again, many other experimental studies did show positive effects of ERT on cognitive functions in naturally and surgically postmenopausal women (Campbell \& Whitehead, 1977; Fedor-Freyberg, 1977; Hackman \& Galbraith, 1976; Resnick, Trotman, Kawas, \& Zonderman, 1995; Sherwin, 1988)

In the present study, the assaciation of ERT-use with memory complaints and cognitive performance was explored using the MAastricht Aging Study (MAAS) (Jolles, Houx, Van Boxtel, $\&$ Ponds, 1995). Hormone-sensitive fluctuations in cognition suggesting early organizational effects of sex steroids on the mammalian brain imply that the group differences between men and women in some specific cognitive abilities are, to a significant degree, a product not only of current but also of early hormonal environments (Kimura \& Hampson, 1994). This suggests specific effects of estrogens on the so-called sexually dimorphic cognitive functions, e.g. on verbal memory, articulation and fine motor skills, but not on spatial memory (Sherwin, 1994). This effect is called the sexually dimorphic effect of estrogens on cognition and it predicts that: ERT in postmenopausal women leads to specific improvements of verbal memory. However, the reduction of anxiety and depression simultaneous with cognitive improvement that is reported as an effect of estrogen treatment suggests that estrogens have a direct tonic effect on the mental state which is independent of vasomotor symptoms (Campbell \& Whitehead, 1977). This effect is 
called the mental tonic effect of estrogens and it predicts general improvement of cognitive functions. In this line, some investigators found nonspecific effects of ERT in improving cognitive functions (Fedor-Freyberg, 1977; Vanhulle \& Demolle, 1976)

In addition, the association of ERT-use with several possible predictors of cognitive function such as age, education, general health risk variables and perceived health variables was explored. Many different variables may be associated with estrogen use and its presumed association with cognitive function and it is uncertain to what extent the effect of ERT is mediated by one or more of these variables. Age and level of education are known to be associated with memory function, sensorimotor speed and cognitive flexibility (Huppert, 1991; Poon, 1993; Salmaso, 1993; Verhaeghen, Marcoen, \& Goossens, 1993). Since several studies also describe that estrogen users in general have a higher level of education (e.g. Tang et al., 1996), age and number of years of education were investigated as potential mediators of ERT effects.

Shaver (1994) suggested that the influence of other therapies and factors (rather than those purely hormonal) on complaints of middle-aged women should be considered as well. The plausible relevant factors they identified were: "perceived stress, mood state, sleeping patterns, disease risk factors, and social patterns'. As such, the influence of perceived heath and health risk factors were also considered in the analyses. Perceived health includes variables such as the number of physical complaints, anxiety score, depression score, insomnia score, and subjective health. Some investigators found that ERT owerall improved the perceived quality of life (Ditkoff, Crary, Cristo, \& Lobo, 1991; Limouzin-Lamothe, Mairon, Joyce, \& Le Gal, 1994; Wiklund, Karlberg, \& Mattsson, 1993). An improvement in perceived health could have an intermediate effect in improving cognitive function or could be independent of cognitive function.

As for health risk variables, there may be specific confounding factors related to estrogen use, since a subgroup can be expected to have received estrogens after surgical removal of the ovaries. Factors to be considered are the number of times subjects underwent general anesthesia and the use of psychoactive medication. Both have been reported to negatively affect cognitive function long after their actual occurrence (Houx, Vreeling, \& Jolles, 1990). Other health risk factors which were seen to have a negative effect on cognitive function include a history of perinatal complications, general angesthesia, brain trauma, brain disease, exposure to neurotoxins, hospital admission, current use of psychoactive medication, current use of tranquilizers and current. tobacco use (Houx et all., 1990).

The rationale of the present study was to explore the association of estrogen use with cognitive performance and to explore the predictive value of ERT in a model with several other independent variables such as age, education, health risk - and perceived health variables on cognitive complaints and cognitive performance in elderly women. The hypotheses were that ERT-users have a higher level of education as compared to controls; that ERT-users have higher scores on perceived health wariables than controls; that ERT-users have more health risk factors than controls; and that ERT-users have better cognitive performance scores (memory, cognitive flexibility, and sensorimotor speed), which are independent of age, education, health risk factors, and perceived health. 


\section{Method}

\section{Subjects}

The MAastricht Aging Study (MAAB) consists of four separate cross sectional panel studies which were randomly drawn from a patients registration of a network of family practices (RNH). These studies share the same methodology with respect to sample frame, subject inclusion and stratification criteria, and basic measurement protocol. Briefly, each subject panel is stratified for age, sex, and an equivalent of general ability. The four panels comprise a total number of 1874 subjects (Jolles et al, 1995) p 25-27). Medical exclusion criteria for the subject sampling procedure are defined as those active or inactive medical conditions in the RNH problem list that may interfere with normal cognitive function. This definition includes the following conditions: coma (only active), cerebrowascular pathology ("CVA'), all tumors of the nervous system, congenital malformations of the nervous system, multiple sclerosis, parkinsonism, epilepsy (all types), dementia, organic psychosis (other than dementia), schizophrenia, affective psychosis, and mental retardation. In addition, before participation in the test program all participants are screened by means of a semi structured interview to update RNH exclusion criteria and to check for the following exclusion criteria not coded in the RNH data base: history of transient ischemic attacks (TIA), brain surgery, hemodialysis for renal failure, electroconvulsive therapy, and regular use of psychotropic drugs. A transient condition, for instance, intercurrent illness or hospitalization, can be a reason to postpone participation. In the first panel, this second screening was performed at home by a research nurse. In the other three panels it was done by tellephone.

In the MAAS database, 23 out of 342 women were identified as ERT-users. They were born between 1929 and 1949 and their age was between 45 and 65 at the moment of study. The date of the actual menopause was not available. On average subjects had taken ERT for 4.3 years. In table $\mathbb{1}$ the different ERT therapies and user frequencies are listed. A selection of females of the population sample was made based upon the age range of the ERT group (range: 45-65 years of age). This selection yielded a group of 319 control (female) subjects. Thus, the ERT-users comprised $6.7 \%$ of the total sample of 342 subjects. Of the ERT users, medical histories were checked by inspecting the status for medical conditions associated with ERT-use, such as hysterectomy and ovariectomy. Unfortunately, these data were not readily available for controls. In table 2, relevant subject characteristics are listed

Talje 2 Subject characteristics: menopawsol history, yedrs of edication and of ERT-wse

Medical History possibly related to ERT-use

\begin{tabular}{|c|c|c|c|c|c|c|c|c|}
\hline years of edicuation & mean & sat. & $N$ & ovariectomy & hyster & ctomy & sterilized & other \\
\hline ElkT Tusers & 10.8 & 2.8 & 23 & 4 & 8 & & 5 & 6 \\
\hline Conirols & 9.8 & 2.3 & 318 & & & & & \\
\hline years of ERT-use & mean & sd. & $N$ & $<1$ year & 2 & -4 years & & $6-23$ years \\
\hline ERT-uisers & 43 & 5.1 & 23 & 8 & & 9 & & 6 \\
\hline
\end{tabular}




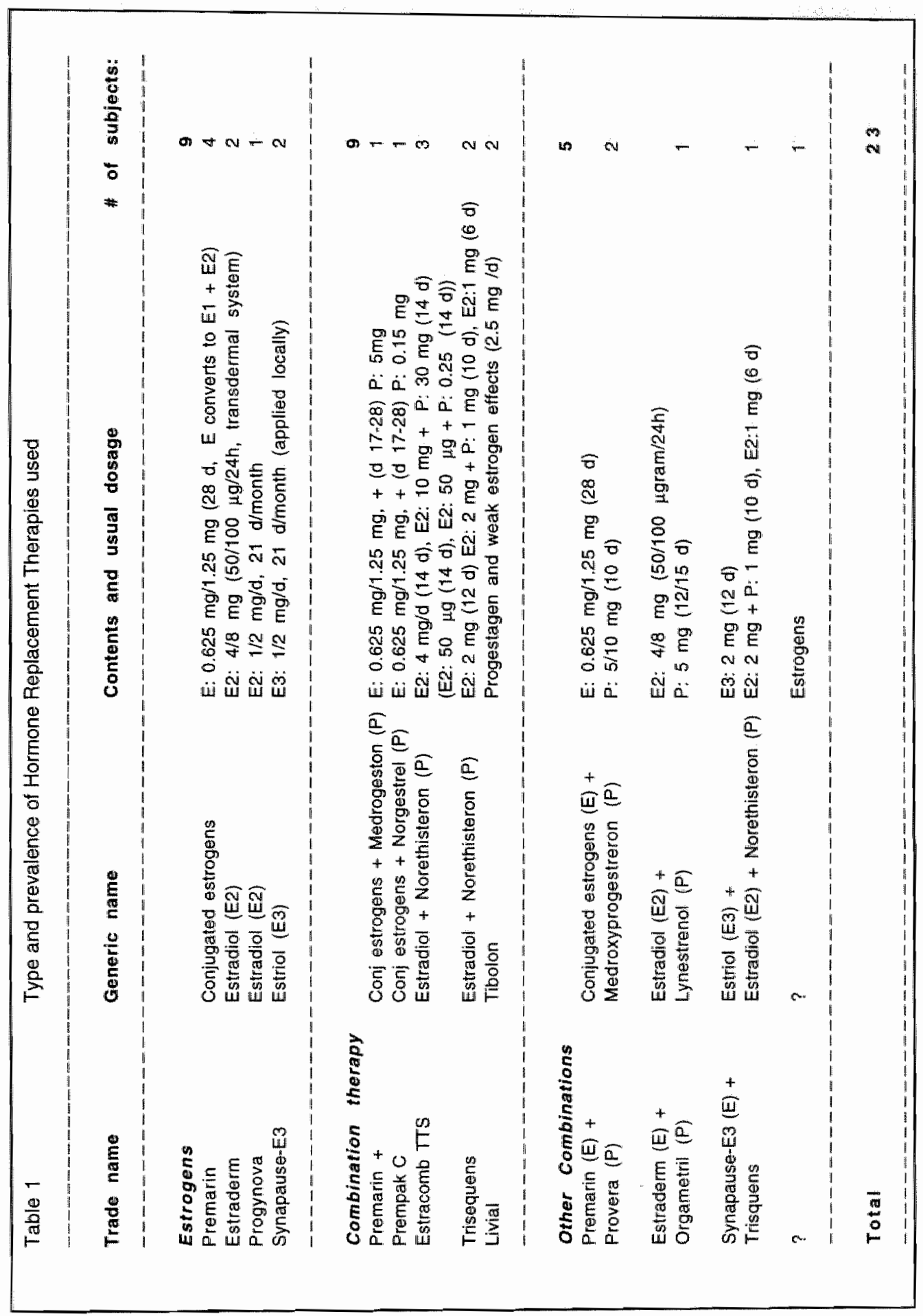




\section{Brocedure}

Tegt sessions were scheduled on work days and Saturdays, in the morning, afternoon, or evening hours. Participants were tested at the neuropsychological laboratory of the Institute Brain \& Behavior (University Hospital, Masastricht). Four separate sound proof rooms permitted three parallel test sessions to be carried out at the same time. Completion of a test session took 2.5 hours on average. In general the time required to test elderly subjects was substantially longer than 2.5 hours.

Five test assistants performed the MAAS data collection. All test assistants received intensive training in test administration and data documentation from the neuropsychologists and physician on the project staff. All assistants performed a complete test session under close supervision of a member of staff at the start of the study and again after 3 months. Frequent debriefing meetings were scheduled with staff and test assistants to discuss problems and other issues in order to maintain the enthusiasm and commitment of the personnel. Senior staff took part in test sessions at least once a week to ensure that they remained sufficiently familiar with the test procedures.

\section{Assessments and tests}

In the MAAS data base, measurements are derived from neurocognitive tests and questionnaires. The questionnaires were used for a postal survey which preceeded the phase of neurocognitive testing and yielded the variables briefly described below. In table 3 an overview of all dependent and possibly confounding variables is given.

Table 3 Overview of all dependent variables

Variables seale range calegories

Cognitive Function

Memory compound seore

Sensorimotor speed compound score

Cognitive flexibility compound score

Subjective Forgettulness

Socio-Demographic variables

Age

Years of Edtacation

Marital Statws

Orlspring

Health Risk factors

History of generml anesthesin

Use of psychoachive medication

History of unaquillizer use

Perinatal Complications

Nistory of britin Itama

Mistory of bratin disease

Exposure to Neurotoxins

Hospital admission (Ever)

Smoker (Present)

Perceived thaluh

Number of physical complaints

Subjectively experienced health

Anxioty Score

Depression Score

Insomnia Score

$\begin{array}{lll}\text { interval } & \text { cont } & z \text {-scores } \\ \text { interval } & \text { cont } & z \text {-scores } \\ \text { interval } & \text { cont } & z \text {-scores } \\ \text { nominal } & 2 & \text { yes, no } \\ & & \\ \text { ratio } & \text { cont } & 4.5 .65 \\ \text { ratio } & \text { cont } & 5.18 \\ \text { nominal } & 4 & \text { newer m., married, divored, widow } \\ \text { nominal } & 2 & \text { no, yes }\end{array}$

$\begin{array}{lll}\text { nominal } & 2 & \text { no, yes } \\ \text { nominal } & 2 & \text { no, yes } \\ \text { nominal } & 2 & \text { no, yes } \\ \text { nominal } & 2 & \text { no, yes } \\ \text { nominal } & 2 & \text { no, yes } \\ \text { nominal } & 2 & \text { no, yes } \\ \text { nominal } & 2 & \text { no, yes } \\ \text { nominal } & 2 & \text { no, yes } \\ \text { nominal } & 2 & \text { no, yes }\end{array}$

$\begin{array}{lll}\text { interval } & 21 & 0 . .21 \\ \text { ordinal } & 5 & \text { very, bad, reasonable, good, very } g . \\ \text { interval } & 41 & 10.50 \\ \text { interval } & 65 & 16 . .80 \\ \text { interval } & 13 & 3 . .15\end{array}$




\section{Cogritive Performance}

Three cognitive performance tests, the Verbal Learning Test, the Concept-Shifting Test and the Stroop Color-Word Test, were taken to yield the main dependent variables: Memory. Sensorimotor Speed and Cognitive Fllexibility. Verbal Leaming Test (VVLT). The 'Groningen Fifteen Words Test' (Brand \& Jolles, 1985; Deelman, Brouwer, Zomeren, \& Saan, 1980), which is well-known in the Netherlands, is an improved version of a test originally devised by Rey (Rey, 1964). Briefy, 15 words are presented, one after another, separated by short time intervals. Then the subject is asked to recall as many words as he or she can. This procedure is repeated five times, so that a learning curve can be plotted. The maximum number of words recalled out of 15 in one trial (vltmax) and the total number of words recalled over 5 trials (viltot) are independent measures of learning and short term memory. After 20 minutes, a delayed recall trial is performed without presentation of the list.. The score at this delayed recall (vitdel) comprises the Jong-term memory score. Concept Shifting Test (CST). The CST consists of three parts. On each test sheet, 16 small circles (diameter $=15 \mathrm{~mm}$ ) are grouped in a larger circle, with a radius of 8 centimeters. In the smaller circles, the test items (numbers $(A)$, letters $(B)$, or both $(C)$ ) appear in a fixed random order. Subjects are requested to cross out the items in the right order. In part $A$ and $B$, the subjects have to connect the numbers (1-2-3-etc.) and letters (A-B-C-etc.) respectively. In part $C$, the subject is required to alternate between these sequences $(1-A-2-B-e t c)$. An exact estimate of the slowing due to the shifting between concepts can be obtained by comparing prant $C$ (digits and letters) with part A and part B (letters). Furthermore, there is a so-called nil-version of the CST which contains only empty circles which have to be connected as fast as possible. The Stroop-Color-Word Test (SCWT) has often been used to test selective attention (Houx, Vreeling, \& Jolles, 1993). The test inwolves three cards displaying a hundred stimuli each: color names, colored patches, and color names printed in incongruously colored ink. The amount of extra time needed to discard irrelevant but very salient information (verbal), in favor of a less obvious aspect (color naming) is recorded.

Three outcome measures were derived from these tests: memory, sensorimotor speed, and cognitive flexibility. Total, maximal and delayed recall scores on the Verbal Learning Task (VVLT) made up the memory score. The null, digit, and letter versions of the CST, and the Stroop Color Word Test yielded an index of sensorimotor speed and the letter-digit version of the Concept Shifting Task (CST) and the Stroop interference were combined to give the cognitive flexibility score (Van Boxtel, Langerak, Houx, \& Jolles, 1996). These measures are compound scores that were calculated from the $Z$-transformed raw test scores, using the formalae:

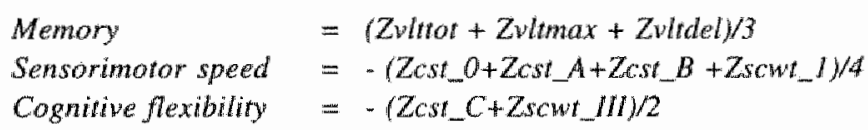

\section{Subjective complaints about memory.}

Subjectively reported memory complaints were obtained by a questionnaire in the postal survey and were derived from the question:"Do you consider yourself forgetful?" The answer could be "no'(1), "yes" (2) or "don't know" (3).

Questionnaires Survey

Sociodemographic variables. This section contains questions about subject parameters. The number of years of full-time education was recorded. Information was also collected about marital status and offspring of the respondents. Data of the onset of the menopause were not available. Only age and years of education were included as confounders in the analysis of the 
weight of the association of the ERT-use with cognitive performance

Health risk factors. Questions covering past and present morbidity that might have relevance for cognitive functioning sere select from a widely used medical screening questionnaire (LHV, 1981). Items were adapted to include information about the type of medicall consultation resulting from the illness or complaint. Medication use (including 'over-the-counter' drugs) was recorded and classified according to its phamacological characteristics and side-effects (custom classification, based on Dukes, (Dukes, 1988; Dukes \& Dijke, 1984). The sum of the variables "history of perinatal complications", "general anaesthesia, 'brain trauma", "brain disease', "exposure to neurotoxins", "hospital admission", "use of psychoactive medication", "current use of tranquilizers' and 'current tobacco use' was entered as a the 'health risk' variable in the analysis of the weight of ERT-use with cognitive performance, since these factors were earlier found to have a negative effect on cognitive functions (Houx et al., 1990).

Perceived health. The subjects were asked to rate their health on a 5-point scale (range: 'very bad' to "very good'). The VOEG (Vragenlijst Omtrent Ervaren Gezondheid - inventory of subjective health) was included as an index of subjective health. It was originally developed by Dirken (1967) to measure stress in industrial situations. It has been used in several Dutch surveys to determine subjective health status. The 21 -item version administered in this study probes health complaints of a somatic and psychosomatic nature. The VOEG-21 was included because of the expected relationship between subjective measures of memory and memory-related functions and subjective health. Because of the expected relationship between mood and memory complaints, three subscales of the Symptom-Check-List 90 (SCL-90) were included (subscales depression, anxiety and insomnia). The SCL-90 is a widely used multidimensional checklist for psychopathological complaints (Arrindell \& Ettema, 1986). The sum of the variables "physical complaints," "anxiety", "depression", "insomnia', and 'subjective health' (reversed) was entered as the "perceived health' variable in the analysis of the weight of ERT-use with cognitive performance.

\section{Statistical Analyses}

The statistical analyses were performed in two steps. The first step was a straightforward comparison of the cognitive-, sociodemographic-, health risk- and perceived health variables, batween ERT-users and controls. ERT-users were compared with controls with respect to sociodemographic background variables." age, years of education, marital status, and offspring. Furthermore, ERT-users were compared with controls on health risk factors. Dependent variables were the following: history of operation under general anaesthesia, use of psychoactive medication, history of tranquillizer use, perinatal complications, history of brain trauma, history of brain disease, exposure to neurotoxins, hospital admission (ever) and smoking, and the sum of these variables ("health risk"). Also, ERT-users were compared with controls on perceived health variables. Dependent variables were the following: number of physical complaints, subjectively experienced health, and the anxiety -, depression -, and insomnia scores of the SCL-90 and the sum of these variables ('perceived health'), Lastly, ERT-users were compared with controls on memory complaints and cognitive performance. Dependent variables were: subjective memory complaints, memory compound score, sensorimotor speed compound sciore, and cognitive flexibility compound score. Nominal variables are tested using Chi2 ${ }^{2}$ tests ordinal variables are tested using Mann-Whitney-tests, whereas variables measured on intervall and ratio level are tested using Students totests.

The second step comprised post-hoc logistic and linear multiple regression analyses. First, ERT use was entered separately. Then, ERT use was entered followed by a stepwise forward entering of 
the age, education, general health risk- and perceived health variables to assess the russociation of these variables, as compared to the weight of the effect of ERT-use, on cognitive function. Independent variables were: ERT-use, age, years of education, health risk factors (sum of history of general anesthesia, use of psychoactive medication, history of tranquillizer use, perinatal complications, history of brain trauma, history of brain disease, exposure to neurotoxins, hospitai admission, and smoking), and perceived health (sum of number of physical complaints, subjectiwely experienced health, and the anxiety -, depression - and insomnia score of the SCL90). Dependent variables were: subjective memory complaints, memory compound scone, sensorimotor speed compound score, and cognitive flexibility compound score. In the logistic regression analyses, the OR (Observed Risk) is given as an estimator of the Relative Risk (RR) to assess the strength of the association between $\mathrm{ERT}$ and the other variables and subjective memory complaints. A summary of the results is shown in Table 4 , which includes the descriptive statistics of the comparisons between ERT-users and controls.

\section{Results}

Table 4 Summary of results of all dependent variables (mean and SD) analyzed for ERT-users vs. Controls

Variables $\quad$ ERT $(n=23) \quad \operatorname{CON}(n=319) \quad$ Statistic p<

\section{Cognative Function}

Mentinory compound score

Cognitive flexubility compound score

Sensorimotor speed compound score

Subjective Forgetfulness (yes)

Socio-Demographic variables

Age

Years of education

Marital status (yes)

Offspring (yes)

Health Risk factors (present) $\Sigma$ History of general anaesthesia Use of psychoactive medication History of tranquillizer use Perinatal Complications History of brain trauma History of brain disense Exposure to Neurotoxins Hospital admission (Ever) Snoker

Perceived Health $\Sigma$

Number of physical complaints

Subjectively experienced health

Anxiely score

Depression Score

Insomnia Score

$\begin{array}{llll}.13(.94) & -.01(.93) & t=-.72 & \\ .30(.59) & -.02(.86)^{n} & t=-1.73 & \\ .33(.60) & -.03(.78)^{n} & t=-2.16 & .05 \\ 59 \% * & 38 \% & c h \%^{2}=3.84 & 0.5\end{array}$

\begin{tabular}{|c|c|c|}
\hline $54.3 \quad(5.8)$ & $55.2 \quad(6.3)$ & $1=.66$ \\
\hline $10.8 \quad(2.8)$ & $9.8(2.3)^{n}$ & $t=-1.94$ \\
\hline $82.6 \%$ & $83.1 \%$ & $\mathrm{Chi}^{2}=.10$ \\
\hline $87.0 \%$ & $88.6 \%-$ & $\mathrm{Ch}^{2}=06$ \\
\hline $2.96(1.4)$ & $2.68(1.3)$ & $t=.98$ \\
\hline $100 \%$ & $86.2 \%$ & $\mathrm{Ch}^{2}=3.64$ \\
\hline $8.7 \%$ & $6.9 \%$ & $\mathrm{CH}^{2}=11$ \\
\hline $17.4 \%$ & $11.9 \%$ & $\mathrm{ChH}^{2}=.60$ \\
\hline $13.0 \%$ & $16.3 \%$ & $\mathrm{Ch}^{2}=.17$ \\
\hline $17.4 \%$ & $19.7 \%$ & $\mathrm{Ch} \mathrm{H}^{2}=.08$ \\
\hline & & $\mathrm{Ch}^{2}=.18$ \\
\hline $9.1 \% \%$ & $48 \%$ & $\mathrm{Cn}^{2}=.77$ \\
\hline $95.7 \%$ & $88.7 \%$ & $\mathrm{Chi}^{2}=1.08$ \\
\hline $26.1 \%$ & $27.2 \%$ & Chit $=.01$ \\
\hline $43.1 \quad(16.4)$ & $49.8 \quad(116.9)$ & $t=-1.84$ \\
\hline $7.4 \quad(3.9)$ & $6.3 \quad(4.5)^{5}$ & $t=-1.08$ \\
\hline $3.7 \quad(.54)$ & $3.8 \quad(.5)^{m}$ & $Z=-.21$ \\
\hline $15.5(4.9)^{*}$ & $13.3(4.9)^{5}$ & $t=-2.02$ \\
\hline $24.98(7.4)$ & $21.78(7.4)$ & $t=-1.99$ \\
\hline $5.59(2.9)^{*}$ & $5.79(2.9)^{a-a}$ & $z=31$ \\
\hline
\end{tabular}

*n=22 (RT), $\wedge_{n}=318, \sim H=317, s n=316, n=315$

ERT-users had had more years of education $(10.78 \mathrm{yrs})$ than controls $(9.79 \mathrm{yrs})\left(\mathrm{t}_{(23.318)}=-1.94\right.$, $\mathrm{p}=.05$ ). Unexpectedly, ERT users overall had a trend for a lower perceived health as compared to 
nonusers $\left(\mathrm{r}_{(2,3,1)}=-1.84, \mathrm{p}=07\right)$. ERT-users were more likely to consider themselves forgetful

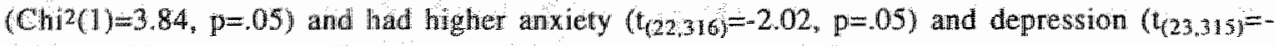
$1.99, \mathrm{p}=05)$ scores. No differences were seen between groups for health risk variables $\left(\mathrm{t}_{22}, 306\right)=$ 98, $\mathrm{p}=\mathrm{ns}$ ). As for objective cognitive tests, ERT-users had a higher sensorimotor speed score $\left(t_{23}, 318,=-2,16, p<.05\right)$, while no effect was seen on the memory compound score $\left(t_{23,319)}=-72\right.$, $p=n s)$ and only a trend was seen for higher scores of cognitive flexibility $\left(t_{23,318}=-1.73, p=09\right)$.

The results of logistic regression anialysis of sociodemographic-, bealth risk- and perceived health wariables on subjective forgetfulness showed that ERT use, when entered separately significantly increased the risk for subjective forgetfulness $\left(\mathrm{Chi}^{2}(1)=4,46, p<05, \mathrm{~B}=-48, \mathrm{SE}=23\right.$, $\mathrm{Wald}_{(1)}=4.30, \mathrm{p}<05, \mathrm{OR}=62$ ). Entering the other variables stepwise forward revealed that women who considered themselves as forgetful, perceived their health less favorable ( $C^{2} i^{2}(1)=22.24$, $\mathrm{p}<0001, \mathrm{~B}=.03, \mathrm{SE}=.01$, Wald $(\mathrm{O})=19,27, \mathrm{p}<.0001, \mathrm{OR}=1.04)$. The effect of ERT was partialled out $\left(\mathrm{B}=-3.8, \mathrm{SE}=24, \mathrm{Wald}_{(1)}=2.43, \mathrm{p}=\mathrm{ns}, \mathrm{OR}=69\right)$. Age, education, and health risk factors were not entered in analysis.

Further, the results of regression analysis of sociodemographic-, health risk - and perceived health variables on the memory compound seore showed that the ERT had a non significant contribution in explaining the variance on this score $\left(R^{2}=.001, F(1,319)=41, p=n s, B=13\right.$, $S E=21, B=.04, t=64, p=n s)$. ERT was partialled out of analyses when education was entered stepwise forward $(B=.07, \mathrm{SE}=.21, B=.19, t=.34, \mathrm{p}=\mathrm{ns})$. Women who performed better on memory tests were higher educated, since years of education was seen to explain $9 \%$ of the variance $\left(R^{2}, F(2,318)=8.11, p<.0005\right)$ on the memory compound score $(B=.09, S E=.02, B=.22$, $t=3.98, p<.0001)$ while the other variables age $(B=-.06, S E=-.06$, tolerance $=.95, t=-1.12, p=n s)$, health risk factors $(B=.01, S E=, 01$, tolerance $=98, t=.17, p=n s)$ and perceived health $(B=-.10$, $\mathrm{SE}=-.10$, tolerance $=.98, t=-1.84, \mathrm{p}=07$ ) were not included in the equitation.

The results of regression analysis of sociodemographic-, health risk- and perceived health variables on the cognitive flexibility compound score revealed a non significant contribution of $\mathrm{ERT}\left(\mathrm{R}^{2}=.01, \mathrm{~F}(1,318)=2.5, \mathrm{p}=\mathrm{ns}, \mathrm{B}=.30, \mathrm{SE}=19, \mathrm{~B}=.09, \mathrm{t}=1.58, \mathrm{p}=\mathrm{ns}\right.$ ). When variables were entered stepwise forward with ERT, age and education were seen to explain $17 \%$ of the variance $(\mathrm{F}(3,316)=21,77, \mathrm{p}<.0001)$ and the effect of ERT was partialled out $(\mathrm{B}=.21, \mathrm{SE}=.18, \mathrm{~B}=.06$, $t=1.19, p=n s)$. Women who performed better on cognitive flexibility tests were younger $(B=-.04$. $\mathrm{SE}=0 \mathrm{I}, \mathrm{B}=-.29, \mathrm{t}=-5.56, \mathrm{p}<.00001)$ and higher educated $(\mathrm{B}=.08 . \mathrm{SE}=.02, \mathrm{~B}=.31, t=4.37$, $p<.00001)$. Health risk $(B=-.08, S E=-.09$, tolerance $=.94, t=-1.64, p=n s)$ and perceived health $(B=-06, S E=-07$, tolerance $=95, t=-1.19, p=n s)$ were not entered in the equitation.

The results of regression analysis of sociodemographic-, health risk- and perceived health variables on the sensorimotor speed compound score showed that ERT use explained only $\rrbracket \%$ of the variance when entered alone $\left(R^{2}=11, F(1,318)=4.11, p<.05, B=.35, S E=.17, B=.11, t=2.03\right.$, $p<$.05]. Age explained $12 \%$ of the variance when entered stepwise forward with ERT. Explained variance was raised to $16 \%$ when years of education was entered on the next step $(F(3,316)=20.17, p<.00001)$. Women who performed better on sensorimotor speed tests were younger $(\mathrm{B}=-.03, \mathrm{SE}=.006, \mathrm{~B}=-.29, \mathrm{t}=-5.45, \mathrm{p}<.00001)$ and higher educated $(\mathrm{B}=.07, \mathrm{SE}=02, \mathrm{~B}$ $=.21,1=3.89 . p<.0001)$. The contribution of ERT-use approached significance $(B=27, S E=16$ $B=.09, t=1.70, p=.09)$. Health risk factors $(B=-.07, S E=-08$, tollerance $=.94, t=-1.36, p=n s)$ and perceived health $(B=-.04, S E=-04$, tolerance $=95, t=-.77, p=n s)$ were not entered in the equitation. Hence, women who scored higher on sensorimotor speed were somewhat more likely to be ERTusers. The performance of women using ERT on sensorimotor speed tasks was comparable to the performance of women that were nine years younger ( $B$ age divided by $B$ ERT). 


\section{Discussion}

When taking into account the small number of ERT-users in the MAAS-database, it may be concluded that considerable differences were found between ERT-users and controls. However, as to the main purpose of this exploratory analysis, the hypothesized effects of ERT on coginitive performance could not be confirmed in statistical analyses when the influence of confounding variables was partialled out, since for memory and cognitive flexibility no effects were found and for sensorimotor speed only a trend significant effect was observed.

We did find, conform the hypothesis, that ERT-users had had more years of education. Other studies also found that ERT-users had higher levels of education (e.g. Tang et al., 1996). Since education is known to affect the performance on cognitike tests and may be a protective factor in age-related cognitive decline, this means that the effect of education can confound the effect of ERT use. However, the trend towards the expected independent positive association of ERT-use with performance on sensorimotor speed tasks was independent of both age and education.

Some of the findings were quite contrary to expectations. For instance, women in the ERT group were shown to complainmore of anxiety and depression. In earlier studies the positive effect of estrogens on mood were extensively described. In fact, it was even considered to be an alternative antidepressant for postmenopausal women (Backstrom, Bixo, \& Hammarback, 1985; Klaiber, 1982). Also, a stabilizing of mood by estrogen use in general has been reported. First, at major flaw in the present study is that data on the menopausal status of women were not available. However, changes in cognitive function and mood have been reported 2 to 8 years before the onset of the menopause (mean age $51.4 \pm 3.8$ years) as the patterns of the menstrual cycle start to change (Bungay, Vessey, \& McPherson, 1.980; Judd, 1987). Thus, it may be expected that the controls in our study were already experiencing the cognitive decline associated with the perimenopause. Analysis which include women of all age-cohorts $(25-35,40-50,55-65,70-85)$ will be carried out to further investigate this hypothesis.

Second, an alternative explanation is that ERT-users were more anxious and depressed due to one of the most likely candidates of indication for estrogen use, namely bysterectomy andor ovariectomy due to endometrial cancer. Cultural differences may explain some of these findings. In the United States of America, more women are reported to use estrogens. For instance, the 15 year prospective, cross sectional study by Barrett-Connor \& Kritz-Silverstein (1993) showed that of 800 women (aged 65-95 years), almost half of the cohort had used estrogen at some time after the menopause and one third were current users. Other studies in the USA (Salamone, Pressman, Seeley, \& Cauley, 1996), the UK (Griffiths, \& Convery, 1995) and Australia (Maclennan, MacLennan, \& Wilson, 1993) report current use incidences of $17 \%, 20 \%$ and $14 \%$ respectively. In the cohort of the present study only about $7 \%$ of the women used ERT, mainly for strict medical reasons. Probably ERT in the MAAS-database was onlly prescribed in more severe cases or after more severe complaints, either or not related to the climacterial syndrome. These findings are compatible with the notion that clinicians in The Netherlands are more reluctant in prescribing estrogens to postmenopausal women.

Subjects using ERT were also seen to complain more of forgetfulness. These findings are quite the contrary to the earlier findings by, for instance, Schneider and Brotherton (1982) who found that women who used estrogens improved on self rated memory measures. However, no control group was used. Yet, a double-blind crossover study by Campbell and Whitehead (1977) also revealed improved memory self reports after estrogens (Premarin) as compared to placebo. Again, another double-blind study by Paterson (1982) showed no improvement on an ordinal scale of self-report measures of memory function. In this study graded mestranol and 
noresthisterone was compared to placebo. In the present MAAS-database study, most women were allso seen to use additional progestiva. The addition of progestagen is necessary to prevent hyperplasia of the endometrium to occur. However, it has been mentioned that the addition of a progestagen may lessen the beneficial effects of estrogens (Dennerstein ${ }^{1987)}$. Differential results could thus also be explained by the different types of ERT used.

Allo, it should be kept in mind that in the studies mentioned, subjects were conscious of participation in scientific research and were informed of the scientists expectations. Since double blind testing of ERT is difficult (due to withdrawal bleeding) subjects might have been sensitive to expectancy effects. The tentative overall explanation of the results is that ERT-users in the MAAS-database probably reflect women with a pattern of related subjective complaints, i.e. of depression and anxiety, and forgetfulness irrespective of whether these are determined by the climacterial syndrome. ERT-use should have decreased these subjective complaints but instead the ERT-users in the MAAS-database show, despite treatment, increased complaints of forgetfullness. In this line, in another study in which women were not aware of the purpose of the study also no effect on memory and complex speed tasks was found. Furthermore, ERT users also reported a lower activation (Schmidt et al., 1996).

Yet, in contrast to the study by Schmidt et al (1996) and the present study, most studies did find an effect of ERT on memory. It is possible that the test (immediate and delayed free word recall) used in the present study was not sensitive to the effect of ERT. For instance, (Sherwin \& Phillips, 1990; Philips \& Sherwin, 1992; Kampen \& Sherwin,1994) found effects on paragraph recall while no effects were found by Kampen and Sherwin (1994) on immediate or delayed word recall. Similarly, Szklo et al (1996) found no effects of ERT use on delayed word recall. Also, no effects of ERT on the immediate recall of digits (digit span) was found (Ditkoff et al, Vanhulle \& Demol, 1976). Craik (1982) showed that elderly subjects perform most poorly on tasks that contain little envirommental or contextual support and that have high processing demands, such as free recall as compared to paragraph recall (Craik, \& Byrd, 1982). The present data suggest that is possible that the more difficult memory tasks that require more processing capacity are not sensitive to the effect of ERT. In this line, cognitive flexibility was also not influenced by ERT in the present study. However, an independent positive association of ERT-use with performance on sensorimotor speed lasks was detected. Similarly, other investigators found an effect of ERT in improving alertness and attentional functions as measured with sensorimotor speed tasks (FedorFreyberg, 1977; Vanhulle \& Demolle, 1976) Possibly, the general alerting effect of ERT explains while memory task with more environmental contextual support, such as paragraph recall can be positively affected, while difficult tasks which require more processing capacity are not sensitive to ERT.

In sum, the findings of the present study point towards a trend in the direction of a general activating effect of ERT-use, rather than a specific association between ERT-use and memory or cognitive performance. The general activating effect of ERT was found to be independent of age. education, perceived health, and health risk factors. 


\title{
Chapter 12
}

\section{A follow-up study into the effects of combined HRT on cognitive function in postmenopausal women}

\author{
E. Hogerworst, W. J. Riedel, M. L. Boshuizen, D. Tisserand, \& J. Jolles
}

\begin{abstract}
The present study was carried out to investigate long term effects of the combination Hormonal Replacement Therapy (HRT: estrogens and progestagens) in post-menopausal women. Subjects were tested during the estrogen-progestogen phase of the cycle. A carefully matched group of post-menopausal women who did not receive therapy was used as a control group $(\mathrm{n}=11)$. The monthly withdrawal bleeding in the HRT group $(n=11)$ precluded the use of a placebo-treated control group. Long term memory functions were seen to be enhanced with the combined use of estrogens and progestagens after 6 and 12 months. Also, both subjectively experienced activation and the general feeling of well being was improved in HRT users as compared to controls as measured with the AD-ACL and the Neurovegetative questionnaires. It was concluded that combined HRT had a positive long term effects on both mennory and a general feeling of well being.
\end{abstract}

\section{Introduction}

Around the age of 50 women experience the menopause, which, as defined by the WHO, is the permanent cessation of the menstruation, resulting from a loss of ovarian follicular activity (Burger, 1996). The menopausal transition, which has a duration of \pm 4 years, is characterized by fluctuating hormone levels leading to typical symptoms such as hot flushes, night sweats, and psychological complaints. An estrogen deficiency caused by the clecreased ovarian function is held responsible for these symptoms (Judd, 1987). As such, the use of estrogen replacement therapy (ERT) or hormonal replacement therapy (HRT) to treat postmenopausal signs and symptoms has become widespread over the past decades. Many experimental studies have shown positive effects of HRT on cognitive functions (both on subjective reports and objective tests) in natural and surgical postmenopausal women (Campbell \& Whitehead, 1977; Fedor-Freyberg, 1977; Hackman \& Galbraith. 1976; Kampen \& Sherwin, 1994; Sherwin, 1988; Sherwin \& Philips, 1990; Sherwin, 1991; Philips \& Sherwin, 1992; Vanhulle \& Demol, 1976).

Sherwin (1994) claimed that there is sufficient evidence to support the contention that estrogens influence cognitive functioning in women and that this is a spectfic effect. Effects on verbal memory, articulation and fine motor skills were found, but not, for instance, on attention or visuospatial memory (Sherwin, 1994; Kampen \& Sherwin, 1994). By contrast, Vanhulle and Demol (1976) found no effects on memory but did find effects on alertness (visual sensitivity on a vigilance task) and attention (Bourdon. Wiersma test) in women using HRT when compared to controls. Fedor-Freyberg (1977) also found non-specific improvements on the performance of attentionall tests, such as the Konzentrations Verlauf Test, and the Stroop test in HRT users as 
compared to controls after a 3 months period. Similar results were seen on an attention test measuring short term memory and reasoning ability, and a choice reaction time test. These findings suggest that there may be a global improvement in functions such as alertness, or subjectively experienced activation, and attention, whch in turn may affect memory performance. The major problem addressed in the present study is whether the effect of HRT is specific; on memory functions only, or whether the effect is of a more global nature. The study therefore addresses the possibility that HRT affects subjectively experienced activation, also called the 'mental tonic effect'. The mental tonic effect also becomes apparent in the antidepressant actions of estrogens (Băckstrơm, Bixo, \& Hammarbåck, 1985). In this line, Klaiber, Broverman, Vogel \& Kobayashi $(1979 ; 1982)$ found that estrogens as compared to placebo decreased symptoms in severely depressed women. Many other studies have shown that estrogens have a positive effect on the general feeling of well being and the overall perceived quality of life (Ditkoff et al 1991 ; Limouzin-Lamothe, Mairon, Joyce, \& LeGal, 1994; Wiklund et al, 1993).

It is at present thus unclear whether the effects of HRT reported on memory occur independent of changes in subjectively experienced activation and general well being. Methodological differences, such as subject selection, the various cognitive tests and different homonal regimes, render clear cut conclusions difficult. For instance, the addition of progestagens in the second half of the cycle phase to eliminate the risk of endometrium hyperplasia due to unopposed estrogens, has been shown to reduce the positive effect of estrogens (Ohkura et al, 1995; Sherwin, 1996). Again, others did not find a negative effect of the addition of progestogen (LimouzinLamothe al., 1994; Kampen \& Sherwin, 1994).

The present study was carried out to investigate long term effects of the combination therapy (estrogens and progestagens) in post-menopausal women. Subjects were only tested during the estrogen-progestogen phase of the cycle. A carefully matched group of post-menopausal women who did not receive therapy was used as a control group. The monthly withdrawal bleeding in the HRT group precluded the use of a placebo-treated control group. Effects of HRT on memory function and aspecific changes in subjectively experienced activation and well being were assessed with a follow up period of one year. The hypothesis was that HRT in postmenopausal women would improve subjectively experienced activation, general well being, and memory functions.

\section{Method}

\section{Subjects}

A group of 11 healthy postmenopausal women who were elected to receive HRT were matched with 11 women who were drawn form the same outpatient subclinical population, but who had decided not to enter the treatment study. Matching was performed for age, postmenopausal complaints, and social class (table 1). The social class system has 5 subclasses $(1=$ high and 5 $=$ low) which are based on the level of education and highest job llevel held by the subject (MEMIC, 1996). This study is part ofl a larger study into the effect of HRT on vesselwall properties.

Women were recruited through a meeting where information was supplied with regard to perimenopausal complaints. The study was approved of by the medical ethical committee of the Academic Hospital and the University of Maastricht. Subjects were informed before the start of the study about all possible adverse effects of HRT and signed an informed consent form. Before entering the study all subjects underwent a complete medical screening. Inclusion criteria were: age $>45$ years, good health, last menstruation between 1 and 6 years ago, and a weight within 
10\% of population norm for their age. Exclusion criteria were: participation in other medication studies, using "yellow label" medication (medication inducing drowsiness/sleepiness), using $>6$ alcoholic beverages/day, and any sensoric or motoric handicaps which may interfere with test performance. Three women dropped out of the study before the end of the study. One subject of the HRT group dropped out because of side effects occurring at $t 5$. The other two women (one of the HRT group, one of the control group) dropped out of the cognitive study but continued with the larger study.

Table 1: Mean agge (SD) and social class (1-5) of HRT users and controls (CON)

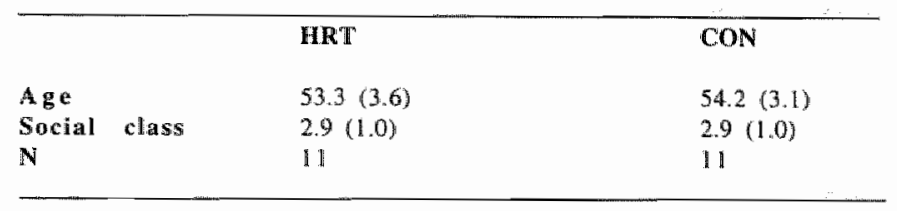

\section{Procedure}

The HRT group received the first 16 days of the cycle $2 \mathrm{mg} \mathrm{17-beta-estradiol,} \mathrm{followed} \mathrm{by} 12$ days of estradiol with additional 2.5 or $5 \mathrm{mg}$ progestogen, during 52 weeks. Subjects received cognitive testing when estrogen and progestogen levels were both elewated (day 16-28). At: baseline (t0) subjects were examined by a medical doctor and, after blood samples being taken, subjects underwent the memory test and filled in the questionnaires. At all following test sessions (6. and 12 months), a similar procedure was followed. Subjects were also tested after 2 weeks, I month and 3 months to eliminate the effect of procedural learning. Data of these test sessions are not shown. At 0,2 and 52 weeks subjects were examined completely by the medical doctor.

Design

This study had a mixed, repeated measures, parallel group design. The within subjects factor was time and had 3 levels. The between factor was HRT use and had 2 levels (HRT and controls). Subjects were tested at baseline (t1), 24 weeks (t5) and 52 weeks (t6).

\section{Cogritive tests}

\section{Primary variables}

Visual Verbal Leaming Test with Distraction A list of 15 monosyllabic, unrelated words was shown by the computer. Every 2 seconds a new word was presented during one second on a computer screen (Brand \& Jolles, 1985). At the same time a list of 15 other words was auditorily presented. Subjects were asked to recall verbally as many words seen on the sereen as possible as soon as the presentation stopped. The first immediate recall was followed by two more trials where the same words were repeated in the same order. When the third trial was completed, the subjects filled in the lists and underwent an interview. After 20 minutes, subjects were asked to recall as many of the previously learned words without prompting (delayed recall). The number of correctly recalled words was noted after each trial. The trials yiliding the total and the maximum number of recalled words were taken as the dependent measures of immediate recall. The number of words contectly recalled after 20 minutes was the dependent measures of longterm memory.

Activation-Deactivation CheckList (AD-ACL) A questionnaire consisting of 20 items, divided in two core dimensions: energy/ positive subjectively experienced activation (activation, 10-40) and tension/ negative subjectively experienced activation (stress, 10-40) (Thayer, 1986). 


\section{Secondary variables}

Naruvegetative Complaints List (NVL) (Bohnen, Twijnstra, and Jolles, 1992) A questionnaire consisting of 26 items which could be scored 1 (never) to 4 (often). These items were grouped into 5 clusters. (1) "Emotional wulnerability" which consisted of the items: Depression (I find that life is a too much a burden for me); Crying spells; Loss of libido; Irritability; Defeatedness; Insecurity; and Tiredness. These ittems had a Cronbach's alpha of 90. (2) 'Psychosomatic-or tension related physical complaints", which consisted of the items: Headaches; Tension; Chest paim; Dyspnoes; Heart palpitations; Dizziness; Wet hands; Problems falling asleep. These items had a Crontrach"s alpha of .79. (3) Cognitive complaints' which consisted of the items: Effort; Slowness of working; Concentration; Difficulty in doing two simultaneous tasks; and Initiative. These items had a Cronbach's alpha of .73. The last two items were (4) Flushes and (5) Quality of sleep which were analyzed separately.

\section{Statistics}

First, dependent variables were analyzed with non-parametric Friedman analyses to assess the effect of the factor time which had 3 levels $(0,24,52$ weeks) per group. If these were significant, with non parametric Wilcoxon analyses, the location of the effect of time was tested (baseline versus 24 and versus 52 weeks, 24 versus 52 weeks) separately per group. Third, at each test moment; the HRT users were compared to controls to investigate the effect of HRT use at baseline, after 24 and after 52 weeks with Mann-Whitney non parametric analyses. P-values < 0.05 were considered significant.

\section{Results}

\section{Primary variables}

Non parametric Friedman analyses revealed that total immediate recall showed a significant effect of time for both HRT users [Chi( 2$)=6.17, \mathrm{p}<.05]$ and controls [Chi( 2$)=9.05, \mathrm{p}<.05]$. HRT use increased the total number of words immediately recalled after $24[z=-2.31, p<.01$ one-tailed] and after 52 weeks $[z=-1.82, \mathrm{p}<.05$, one tailed] as compared to baseline (see fig. 1).

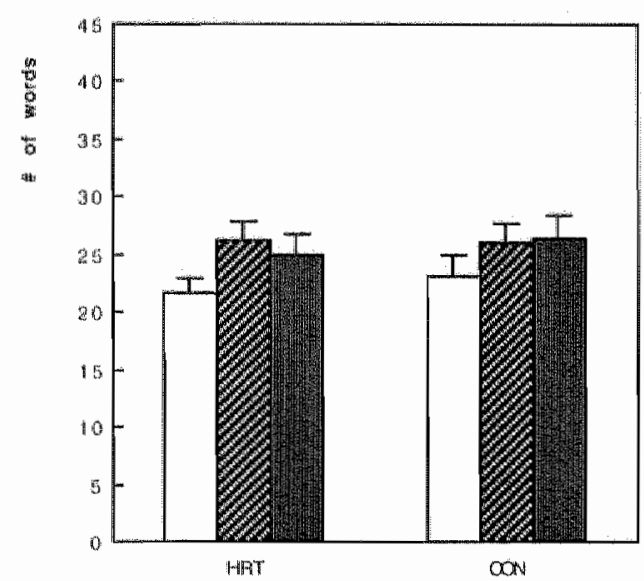

Fig 1. Total of number of words immedialaty recalled OVar 3 trials of the VWL in HRT users and controls at basoline $(11)$, alter $24,(15)$ and after 52 weeks (16)

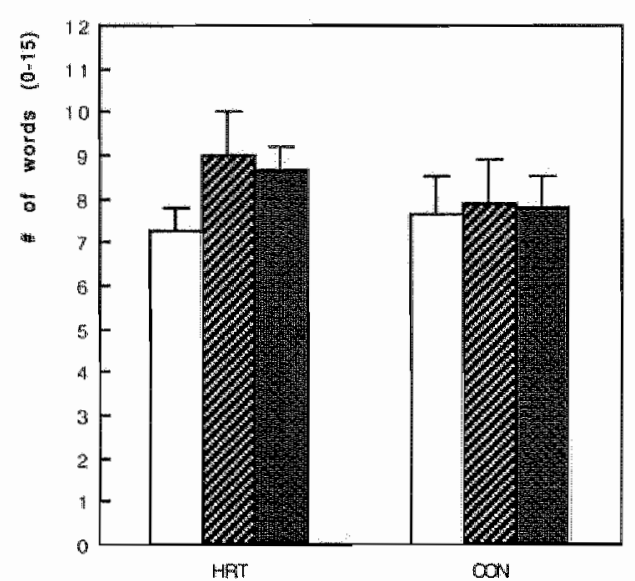

Fig 2. Number of words recalled after a detay of 20 min in HAT users and controls at baselline (11). after $24(15)$ and after 52 weeks (16) 
On average, two more words were recalled after 24 weeks of HRT use. However, also for controls; an increase in the number of words recalled was seen after $24[z=-255, p<01$, two- tailed] and after 52 weeks $[\mathrm{z}=-1.99, \mathrm{p}<05$, two-tailed] (see figure: 1 ).

For delayed recall, non parametric Friedman analyses showed a trend effect of time in HRT users $[$ Chi $(2)=5.06, p=.08]$, but not in controls [Chi( $2 y=67, p=n s]$. Wilcoxon analyses revealed that HRT users recalled more words after $24[z=-1.89, p<.05$, one-tailed] and 52 weeks $[z=-1.86$, $\mathrm{p}<.05$, one tailed $\mathbb{l}$ as compared to baseline. No significant differences were found between HRT users and controls at the different test moments with Mann-Whitney tests (see figure 2).

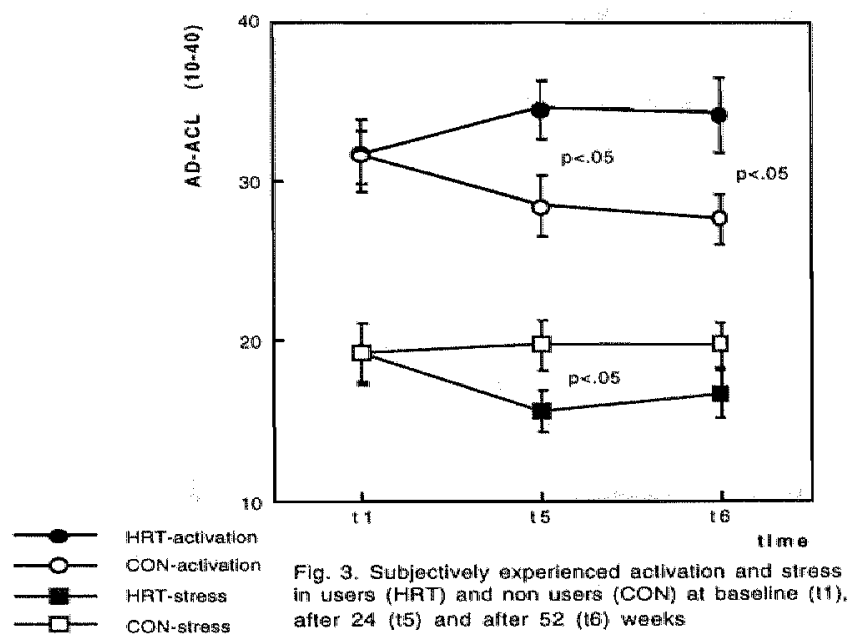

On the activation subscale of the AD-ACL, no effect of time was seen after HRT use [Chi $(2)=.72$, $\mathrm{p}=\mathrm{ns}$ ] or in controls [Chi( 2$)=2.40, \mathrm{p}=\mathrm{ns}$ ] with non parametric Friedman tests. However, MannWhitney analyses revealed that at 24 and 52 weeks, HRT users reported to feel significantly more vigorous and activated as compared to controls $[z=-2.13, p<.05$, two-tailed; $z=-2.26, p<.05$, two tailed] (see figure 3). The stress subscale showed similar effects (see figure 3), as no effect of time was seen after HRT use [Chi( 2$)=1.06, p=n s]$ or in controls [Chi $(2)=15, p=n s]$. Yet, ManmWhitney tests showed that subjects which used HRT were significantly less stressed as compared to controls after $24[z=-2.05, p<05$, two-tailed] but not at 52 weeks $[z=-1.52, p=n s]$.

\section{Secondary variables}

On the neurovegetative scale no differences on the subscales were detected between groups at baseline as separate Mann-Whitney tests revealed. For emotional vulnerability (figure 4) no significant change in scores was seen with Friedman analyses over time for both groups [HRT, Chi(2) $=1.79, p=n s ; C O N$, Chi(2)- $89, p=n s]$. However, HRT users had a significantly lower score on the emotional vulnerability subscale as compared to non users after 52 weeks as Mann Whitney tests showed $[z=-1.94, p<.05]$, while no differences effects between groups were apparent at baseline $[z=-.62, p=n s]$ or after 24 weeks $[-1.33, p=n s]$. Psychosomatic complaints (figure 5) and cognitive complaints (figure 6) also did not show a significant change over time in either group [psychosomatic: HRT, $\mathrm{Chi}(2)=3.5, \mathrm{p}=\mathrm{ns}, \mathrm{CON}$ : Chi(2)=1.5), $\mathrm{p}=\mathrm{ns}$; cognitive: HRT, Chi (2) 
$=2,64, \mathrm{p}=\mathrm{ns}, \mathrm{CON}$. Chir $(2)=1.9, \mathrm{p}=\mathrm{ng}$. However, after 24 weeks HRT users had trend significantly less cognitive complaints as compared to non users as Mann-Whitney amalyses revealed $[z=-1.65$, $p=10]$ which became significantly different between groups after 52 weeks $[z=-2.19, p<05]$.

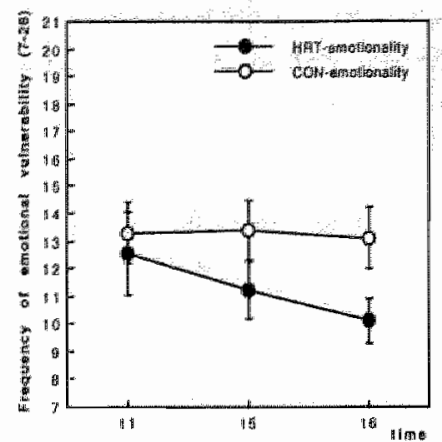

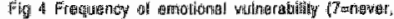
Q in

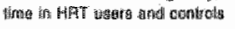

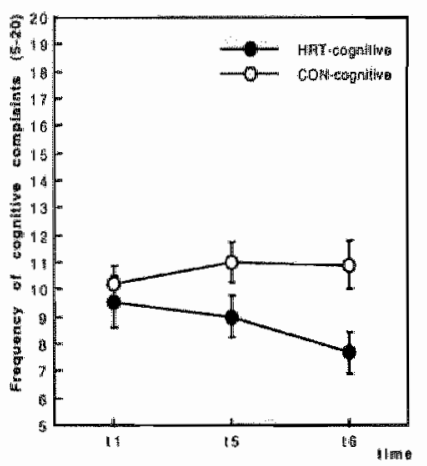

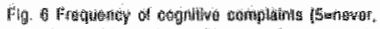

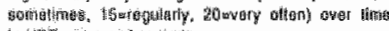

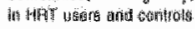

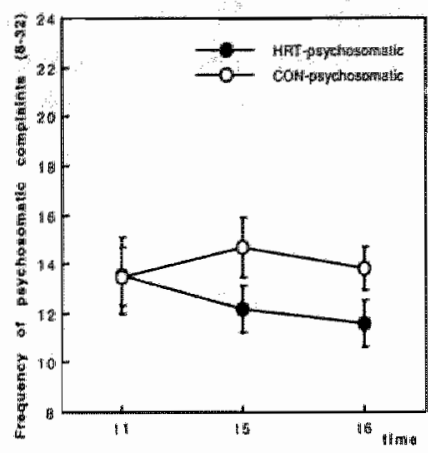

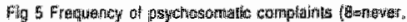
1 10

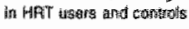

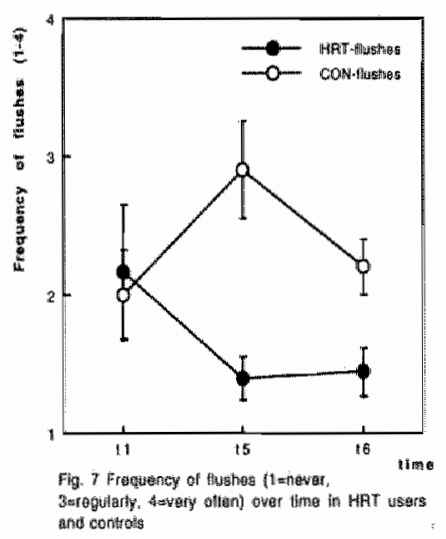

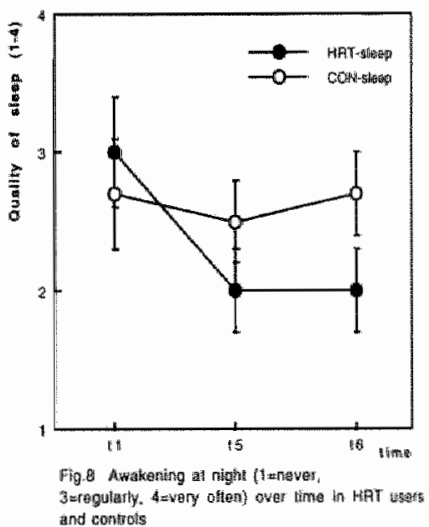

There was no effeet of time on the frequency of flushes (figure 7) in HRT users [Chi(2) $=86$, $p=n s]$ or controls $[C h i(2)=3.56, p=n s]$. Mann-Whitney andyses indicated that the frequency of flushes was similar at baseline $[z=-.95, p=n s]$, but was significantly increased in non users as compared to HRT users after 24 weeks $[z=-2.85, p<.005]$ from sometimes (2) to regularly (3) on average, a difference that still was apparent after 52 weeks $[z=-2.39, p<05]$. Sleep quality (figure 8) showed a trend for improvement over time in HRT users as Friedman analyses showed [Chi(2) $4.5, p=.10$ ] while in non users no difference was seen over time [Chi( 2$)=72, p=n s]$. HRT subjects hit a better sleep quality after 24 weeks and after 52 weeks as compared to baseline as Wilcoxon analyses revealed [resp. $z=-1.83, p<.05$ (one-tailed); $z=-2.02, p<.05$ ]. There was no significant difference between groups, however, as Mann-Whitney tests revealed, only after 52 weeks, a trend signifficant difference was seen for a better sleep quality in HRT users as compared to non users $[z=-1.75, p=.08]$. 


\section{Discussilon}

In the present study, long term memory functions were seen to be enhanced with the combined use of estrogens and progestagens after 6 and 12 months. Also, both subjectively experienced activation and the general feeling of well being was improved in HRT users as compared to controls as measured with the AD-ACL and the Neurovegetative questionnaires. HRT users were less stressed and more activated. Furthermore, after one year of use, HRT users showed less emotional vulnerability and had less cognitive complaints. HRT users showed trends for experiencing less psychosomatic complaints and a better quality of sleep. As expected, they had much less hot flushes. Hypotheses were thus confirmed in that women using HRT had improvements in long term memory functions but also reported global effects in an increase in subjectively experienced activation and general well being.

There are several possible problems in the present study. First, the drop-out of several subjects can be suspected to have confounded statistical effects. However, the analyses concerned with within subjects effects, over time, only compared the subjects that remained in the sample. Hence, this argument does not hold for the effects on the memory task. Also, reanalyzing the ADACL and neurovegetative data without the drop-outs still revealled no significant difference for the baseline values. Second, the expectancy of positive HRT effects may have confounded results. When investigating naturally postmenopausal women, double blind procedures camnot be maintained. The studies performed by Sherwin usually included relatively young groups of women which had undergone hysterectomy, which enabled her to study the effects of estrogens double blind. Yet, we expected that in the present study, the repeated testing ( 6 times in total) may have decreased the expectancy effect. Also, the no-nonsense attitude of the women investigated did not give rise to the suspicion of strong effects. Again, an earlier study, which included a large population sample, showed that women using HRT, which were unaware of the purpose of the study, did not mention any positive effect of HRT on memory self-report measures or on mood questionnaires (Hogerworst et all, in preparation). Hence, it remains to be seen whether the reports of enhanced subjectively experienced activation and well being in the present study were not induced by expectancy effects. A third problem is that learning effects may be responsible for the results found. The study by Rauramo (1975) is illustrative in this matter. In this study no effects were found on cognitive functions. Rauramo (1975) used a parallel groups design with measurements after a 1 and a 6 month period. Similar to the present study, effects of HRT were evident on neurovegetative symptoms, stch as perspiration, flushes, tachycardia and dyspnoea. Also, HRT subjects had a better mood (less tearful and irritable) as compared to the placebo group. Yet, in this study no effects were found on the Integrative Memory Test, on reaction times (RT), on letter cancellation, or on the logical reasoning, as measured with the Raven's Progressive Matrices. The tests used in this study may not have been sensitive to change. For instance, RTs often show no effects, since there is a large intraindividual spread in performance unless subjects are thoroughly trained. The Raven's test is an intelligence test, which is considered to test crystallized intelligence functions, which are not sensitive to change. Also, the plots revealed that learning effects in this study may have confounded results. Similarly, in the present study, procedural learning effects on the immediate recall were detected. It may be expected that the women in both groups employed better strategies in learning the word lists. Yet, the learning effect did not affect the delayed recall parameter. Howewer, effects on the delayed recall (long term memory) performance were only seen when HRT users were compared to their own baseline performance, but not as compared to controls. Hence, fourth, the small number of subjects investigated in the present study could explain the absence of significant differences between 
groups. Robinson et (1994) suggested that the larger variability in estrogen using women (average age $67 \pm 6.5$ years) was responsible for their lack of significant findings on the Object Recall memory test., since they found that the overall mean scores of this test were slightly higher in IHRT users as compared to age and education matched controls. Furthermore, they found that estrogen using women did differ significantly on the Name Recall test. The authors suggested that interindividual differences in the response to ERT may be caused by the differences in the dosage used, the pharmacokinetic responsiweness, age, personality, intelligence or other psychobiological factors. Again; the memory test used in the present study has earlier been shown to be very sensitive to small age-differences, which explains why slight changes within subjects could be detected (Hogervorst et all, in preparation).

Another explanation for differences in findings could lie in the designs employed in the studies. In our earlier study and in the study by Robinson et (1994), in which no or little effect of HRT use on memory were found, a case-control design was used. Similar to the present study, no effects could thus be detected between groups. Using a cross-over design with a within subjects comparison, as was done by Sherwin (e.g. 1988), may be a more sensitive method to detect effects on memory. The present study may indicate why some studies reported beneficial effects of HRT on memory functions, while others could not find effects. Thus, it is likely that the between subjects effects of HRT are quickly overnuled by non specific interindividual variation in performance. Since within subjects design are more sensitive to slight change, minor changes are more easily detected. Again, no beneficial effect of HRT in preventing cognitive decline was seen after a 15 year follow-up period in elderly women (Barret-Connor \& Kritz-Silverstein, 1993). In this study multiple regression analyses were performed involving estrogen use (current, past and never) which was adjusted for age and education. Yet, this study involved very old subjects (8094) and a closer look at the graphs show that HRT seemed to attenuate the deterioration between the ages of 70 and 80 most apparently on a word recall rest and a Fluency test (retrieval of animal names). It is possible that separate analyses within and/or between subjects within an age-cohort would have shown significant differences on verbal memory test performance. In this respect, Kampen and Sherwin (1994) did find effects between subjects, testing one-tailed in a small population sample of normal postmenopausal women, on average aged $65 \pm 5$ years. 21 women used HRT and were compared with 43 equal controls in terms of age, education, socioeconomic status, and age at menopause. However, only one memory test (paragraph recall) differed between groups while the other 13 tests, including two werbal memory tests, i.e, word list recall and paired word associates, showed no differences. As earlier suggested, the lack of findings may have been catised by the between subjects design. However, the authors concluded that the effect of HRT is very specific and not global. Furthermore, in earlier studies (e.g. Philips \& Sherwin, 1992) verbal memory was reported to be enhanced in the absence of an improvement in mood, which was also suggestive of specific effects.

In contrast, in the present study, it was hypothesized that an increase in subjectively experienced activation and well being could indirectly affect memory functions. The literature describes that progestagens reverse some of the effects of estrogen when both are given together. Progestagens are reported to dampen mood (Ohkura et al., 1995; Sherwin, 1996). Further, progestagens are suspected to have antesthetic properties, since they can act upon the GABA system, which decreases the activity of excitatory neurotransmitters (Das \& Chandhuri, 1995). In this respect, Bäckström et al. (1985) reported that progestagens decrease the brain excitability. Furthermore, Merryman found that progestagens may induce sleepiness (in: Sherwin, 1996). However, in the present study, progestagen did not oppose the effect of estrogens or made subjects worse, since subjectively experienced actiwation and well being were not decreased. Similarly, others did not 
find a negatiwe effect of the addition of progestagen on well being (e.g. Limouzin Lamothe et at., 1994). Yet, it should be kept in mind that the increase in subjectively experienced activation and the decrease in stress experienced was rather small and non significant as compared to baseline. Furthermore, the frequency of occurrence of the other subjective complaints was on average "less than sometimes, in both groups. Hence, also controls did not show severe clinical impairment. These findings are in line with the earlier statement by Sherwin (1994) that postmenopausal women are not clinically impaired to a degree that affects their daily functioning. Furthermore, it was suspected that expectancy effects may have induced the subjective parameters. And, contrasting to the effects of memory, there was no within subject difference in subjectively experienced activation or general well being. Hence, due to the nature of these effects, it remains possible that the effect of estrogens on memory functions is independent of subjectively experienced activation and general well being.

As for the effect of combined HRT, many of the positive biochemical effects that estrogens exert on the brain are reversed by the addition of progestagens, such as the reversal of the antiatherosclerotic effect and regulatory effects on cerebral blood flow of estrogens (Sarrel, 1990), the downregulation of the dendritic outgrowth in the hippocampus stimulated by astrogens (Woolley \& McEwen, 1993), and the reversal of the positive effects of estrogens noradrenergic neurotransmitter systems (Holzhauer \& Yondin, 1973; Petitti et al., 1990). Toran-Allerand et al (1.992) demonstrated that estrogen receptors are co-localized with nerve growth factor (NGF) receptors and hypothesized that estrogen-neurotropin interactions may be important for the survival of cholinergic neurons in aging. Again, continuous estrogen treatment was seen to result in a down regulation of NGF and NGF receptors, which may counteract the initial beneficial effects on basal forebrain cholinergic functions (Gibbs, 1994), Yet, the author hypothesized that the combination therapy of estrogens and progestagens may help maintain the positive effects on cholinergic systems. Furthermore, in another study it was found that both estrogens and progesterone can protect cultured hippocampal neurons against glutamate toxicity, glucose deprivation, $\mathrm{FeSO}_{4}$ toxicity, and amyloid B-peptide toxicity. These factors may contribute to neuronal degeneration in various age-associated disorders (Goodman, Bruce, Cheng, \& Matson, 1996). Both the hippocampal and basal forebrain cholinergic systems are thought to be involved in memory functioning (Bartus, Dean III, Beer, Lippa, 1982).

These findings are thus relewant for age-related memory impairment and Alzheimer ${ }^{*} s$ dementia. In this line, with respect to the clinical relevancy of the findings of the present study, a substantial average increase of two words on delayed recall of the word learning test was seen within HRT users. Also, Kampen and Sherwin (1994) found that the addition of progestagens did not alter the effect of estrogens on memory. In addition, several studies have shown that the risk to develop Alzheimer's dementia is significantly reduced in women using HRT (Henderson et al, 1994: Paganini-Hill and Henderson, 1994; Tang et all, 1996) and that women afflicted with Alzheimer's disease, which have used HRT, had superior performance on several memory tests as comptred to non-users (Fillet et al, 1986; Henderson et al, 1996; Ohkura et al, 1994b). Yet, also an absence of protective effects of estrogens in AD were found (Broe et al, 1990; Fillet, 1994) and some early studies suggest that the cognition entancing effects of estrogens are not maintained after 12 months of treatment (Caldwell, 1952 ; Kantor et al, 1973). Similarly in the present study, a slight non significant reversal of effects on memory was seen after 12 months. In sum, long term studies $(>1,5$ years) involving a larger number of subjects of an age group between 65 and 80 years, are needed to investigate the actions of combined HRT in preserving cognitve function. 


\section{Chapter 13}

\section{Concluding remarks}

\subsection{Introduction}

The central issue of this thesis concerns the evaluation of functional physiological, pharmacological, and behavioral human models. These models are used to test the influence of cognition enhancing substances for age-related cognitive decline. A functional model includes two or more levels of a controlled experimental condition, intended to simulate age-related. cognitive decline (e.g placebo=young, scopolamine=old). The rationale is that if the model mimics age-related cognitive decline, the influence of cognition enhancers for the treatment of age-related cognitive decline can be tested in homogenous samples of young or middle-aged healthy volunteers.

\subsection{Questions addressed in this thesis.}

Several experiments were undertaken to test human models for age-related cognitive decline. Caffeine was used as a standard drug, since it has been stated that a cognition enhancer should have effects which are at least greater than the effects of caffeine (Riedel et al., 1995a). In a separate part of this thesis, the effects of hormonal replacement therapy on cognitive function in elderly women with and without dementia were evaluated. Several questions were posed in this thesis. The major findings and their possible implications are summarized and discussed in this chapter.

1. Which cognitive tests should be used to measure the effect of age-related cognitive dectine Elderly subjects are believed to be especially impaired on complex cognitive tasks due to a decrease in mental resources (Jolles, Verhey, Riedel \& Houx, 1995; Kok, Looren de Jong \& Van Rooy, 1988; Salthouse, 1985). By definition, cognition enlvancers should be able to attenuate or reverse these resource-telated deficiencies (Spiegel, 1995). In chapter three, a population sample based study was presented in which performance of a complex memory task with distraction wats compared with performance of a regular memory test in 4 age-groups. It was found that performance on the complex memory task was additionally impaired in the aged groups. Hence, this task was considered sensitive to measure age-related cognitive decline. Furthermore, cognitive slowing and subjective, but not objective, health explained the age-related variance in memory performance. In another study, which involved a healthy self-selected sample, we again found the age-related slowing of response times and the memory retention deficit on the complex memory task (chapter 4). However, the complexity of the task did not induce the additional age-related decrease in memory performance. Hence, healthy subjects (both objective and subjectively experienced) do not experience the additional age-related detrioration in complex memory tests. In the consequent studies, a cognitive test battery was used which consisted of the revised complex memory task with distraction and several RT tasks to measure the speed of information processing. 
2. Which models can be used to mimic age-related cogniture decline and to test potental cognition enhancers?

A. large review was given in chapter two which evaluated the potential of several models for agerelated decline. It was concluded that some of these models may be adequate for age-related cognitive decline, but possibly not for dementia. For instance, scopolamine, a cholinergic antagonist, is used as a model since memory deficits similar to those seen in aging but not dementia, have been found (e.g Riedel et al, 1995). Yet, drugs that were tested using the scopolamine model have proven in treatment studies that the model has a good predictive value for treatment of AACD and dementia. Again, drugs that do not affect the cholinergic system directly have also been seen to attenuate scopolamine effects (see chapter 2). Hence, scopolamine may not be a method to test whether a drug directly acts through cholinergic systems. Using different models may illuminate through which pathway a drug is most likely to exert its main effects on cognitive function. However, other potential neurotransmitter models (e.g. the serotonin depletion model) or combination models have not been tested thoroughly and still await evaluation.

Experimentally induced mild hypoxia is another model that is currently used to detect the effect of potential cognition enhancers in young, healthy volunteers. Although chapter 5 shows that in this model no memory deficits were seen, slowing of performance was apparent in complex tasks which required visual detection and response inhibition. In the same experiment, bypocapnic hyperventilation, a potentially new model, was not shown to be a better model than the hypoxia model for the age-related decreased availability in brain oxygen and the consequent cognitive dysfunction. The slowing of performance during hyperventilation could be mainly attributed to the effect of overbreathing and wearing a mask. A possible relation between the effects of overbreathing and age on a slowing of performance is as follows. During overbreathing subjects reported many physiological symptoms and did not feell well, which indicates that they were not in a state of optimal well being. Chapter 3 showed that subjective experienced health is a factor in explaining the age-related cognitive decline. Elderly subjects can be expected to experience less subjective well being or vitality due to a higher incidence in morbidity (Bots, Pols, De Jong, Grobbee \& Hofman, 1996). These findings may indicate that it is not the actual level of health, but the experience of a lowered subjective well-being that interferes with cognitive functioning.

Lastly, a problem in some models is that cognitive deficits can be overcome in young subjects if they invest more effort. "This was seen in the model of physical exhaustion in which athletes were seen to overcome the effects of physical exhaustion (a time trial) and ewen had a better performance as compared to baseline performance (chapter 6 \% Similar effects are also seen after sleep deprivation, for instance (chapter 2). Motivational and expectancy factors may then confound results. Certain EEG parameters (Event Related Potentials) can be used to clarify whether and when subjects invest additional effort. Also, subjects of middle-aged can be tested in models of cognitive deficiency. This group can be expected to have more difficulty to overcome an induced cognitive deficiency, since decrements in cognitive function are already seen in the fourth and fifth decacle (chap. 3).

3. Is caffeine a valid "golden standard" drug to evaluate the effect of a potential cogmition enhancer in models of age-related cognitive decline?

Chapter 7 showed that caffeine, in middle-aged but not in young or old subjects, enhanced working memory functions. Also, middle-aged subjects are known in general to consume more coffee, possibly in order to compensate for age-related cognitive deficiencies (Riedel, Hogervorst 
\& Jolles, 1995b). In the scopolamine study (Riedel et al, 1995a), caffeine was seen to attenuate the induced memory dysfunction. One explanation is that caffeine acts in states of cholinergic deficiency. However, this does not explain why in two srudies (chapter 7 and 8), a sinuillar dose of caffeine in young subjects was shown to have detrimental effects on menory functions. This issue needs further clarification.

\section{- Caffeine and arousal}

Possibly, the effects of caffeine can be explained by the Yerkes-Dodson law (1908), which states that arousal has an inverted $U$-shaped effect on cognitive functions. Dose-dependent effects of caffeine were seen to occur in in chapter 9 , in which \pm 100 mig of caffeine enhanced memory functions in young subjects, while chapter 7 and 8 showed detrimental effects of $230 \mathrm{mg}$ on working memory functions in young subjects. Furthermore, caffeine is known to be especially effective in states of lowered arousal, such as after sleep depriwation (Lorist, Snel, Kok \& Mulder, 1994). Already in the former century Kraepelin (1899) listed scopolamine to be a 'calming' agent (i.e. arousal lowering). Hence, in accordance with the Yerkes-Dodson law, caffeine in the scopolamine model could have acted positively on memory functions by reversing the decreased arousal. This may be underlined by the caffeine-induced attenuation of the dectements in visual sensitivity after scopolamine, since a decrease in wisual sensitivity may be indicative of lowered arousal (Sanders, 1983). Furthermore, during stressfull inductions (i.e. entanced arousal), a similar dosage of $230 \mathrm{mg}$ caffeine was seen to have a negative effect on long term memory functions (chapter 8). However, in the study of the model of physical exhaustion (chapter 9) caffeine was seen to elevate cognitive functions on top of the positive effect of excercise on cognition. The Yerkes-Dodson model would have predicted, similarly to the effects seen in hypoxia and hyperventilation, negative effects of caffeine after exercise, since subjects were still highly activated. Yet, this did not occur.

\section{- Caffeine, the interpretarion of arousal, and cognitive performance}

Possibly, arousal is too aspecific a term. Important in this respect may be the situation, the state of the subject, and the consequent interpretations of the additional arousal due to caffeine consumption (Schachter \& Singer, 1962), which may lead to different (cognitive) behaviors. Subjects did not feel well during hypoxia, while wearing a mask, and while hyperventilating and may have interpreted their state to be potentially threatening due to the situation (claustrofobia, pain, suffocation). Consequently, subjects would experience negative arousal, or distress. In these situations, the additional arousal through the consumption of caffeine could lead to further distress and consequently be detrimental to long term memory task performance. Subjects under the influence of scopolamine also did not feel optimal (dry mouth, nausea, drowsiness). However, since they were underaroused (similarly to the effect of sleep deprivation or use of benzodiazepines), subjects would have interpreted the additional arousal through the consumption of caffeine as positive and consequently showed an enhancement of cognitive functions. Athletes have positive expectations (reward) of physical exercise (chapter 6). The attribution to the additional aspecific increase in arousal through the consumption of caffeine then is of a positive nature and would consequently lead to an overall enhancement of cognitive functions. This theory is in line with the hypothesis of other investigators (Davinson \& Parrot, 1997) who suggested that the effect of certain drugs, لike XTC or LSD, on information processing, (i.e. bad or good trips), may be caused by a heightened sensivity to external (situational context) and internal (emotional state / expectancy) stimulus conditions. 


\section{- Caffene and newrotransmitier systems}

An alternative biochemical hypothesis of the effects of caffeine on cognitive performance in the physical exhaustion model, in moderate dosages in the middle-aged, in low dosages in the young. and in states of a lowered arousal, such as after the adminstration of scopolamine or benzodiazepines, mat be found in the actions of caffene on several excitatory neurotransmitter systems, not just cholinergic, but also monoaminergic, and possibly glutaminergic systems (Nehlig, Daval \& Debry, 1992), One interesting hypothesis is that the action of caffeine is through affecting monoaminergic neurotransmitter systems. Testing caffeine in models of cognitive dysfunction which affect monoaminergic systems may prove relevant to test this hypothesis.

Lastly, with respect to the possible neurotransmitter-related mechanisms underlying the effects of caffene, it was suggested that caffeine mainly acts in states in which a deficiency is apparent. According to a review by Davis and Bailey (1997) physical endurance exercise induces mental fatigue. The onset of mental fatigue is not necessarily followed by cognitive deficiencies, since subjects first invest more effort to overcome the detrimental effects of fatigue. Several studies have shown that mental fatigue is associated with central cholimergic and dopaminergic deficits. Caffeine has stimulating effects on these neurotransmitter systems and may thus have aided subjects to overcome the perceived exercise-induced deficiencies. Similarly, in subjects of milddle-age, caffeine could reverse neurotransmitter deficiencies. Possibly, structural deficits in the elderly may reduce the profit from the cognition enhancing properties of caffeine. Alternatively, they may need a higher dosage before effects of caffeine become apparent. In situations without apparent neurotransmitter deficits (chapter 7, 8, and 9 before exercise), an enhancement of these neurotransmitter systems would then have a curve-lineair dose-dependent (inverted $U$ curve) effect on cognitive functions, in that a low dose ( $\pm 100 \mathrm{mg}$ ) has a positive effect on memory functions and that a higher dose $( \pm 230 \mathrm{mg})$ has detrimental effects on memory functions.

\section{Caffeine as a golden standard drug}

Can caffeine still be considered a good standard drug? As for the predictive value, caffeine was shown to have positive effects, especially in subjects of middle-age. In many textbooks caffeine is listed with psychostimulants, such as amphetamine. Stimulants, such as metamphetamine, are known to positively affect learning and memory consolidation functions (Soetens, Casaer, D'Hooge \& Hueting, 1995; Soetens, DHooge \& Hueting, 1993). In this line, stimulants have been considered as treatment of age-related cognitive decline and dementia. Still, the risk for adverse side-effects on the nervous and peripheral system with the prolonged use of stimulants needs to be further evaluated. Here, animal studies may be of potential value.

\section{What is the nature of the effect of hormonal replacentent the rapy on age-related cognirive decline?}

Many argue that models of age-related cognitive decline are not of value to test cognition enhancers since these should focus on the maintenance of physiological systerns and protect against a structural decline (Gamzu, Birkhimer, Hoover \& Gracon, 1990). Hormones may be a new area in this field; especially gonadal replacement therapy in elderly women is mentioned in this respect. A review was presented in chapter ten, in which the effects of hormonal replacement therapy (HRT) in elderly thealthy and demented women were critically discussed. Although epidemiological studies indicate that the risk of developing Alzheimers' Disease is reduced when using HRT (Henderson, Paganini Hill, Emanuel, Dunn \& Buckwalter, 1994; Tang et al., 1996), 
this does not indicate a causal relation. The studies that involved demented subjects have nany naws (Fillit et al., 1986; Ohkura et al., 1994). Also, a longitudinal study with normal subjects did not show that HRT prevented the age-related decline (Barrett Connor \& Kritz Silverstein, 1993). A reason for different results in HRT research could be that different homone regims were used. For instance, the effects of the addition of progestagens have been mentioned to oppose some of the effects of estrogens (Backstrom, Bixo \& Hammarback, 1985; Sarrel, 1989; Woolley, Gould, Frankfurt \& McEwen, 1990). Long term effects of combined HRT use (estrogens and progestagens) have not been systematically investigated.

In chapter 12, a study into the long term effects of combined HRT on normal postmenopausal women was presented. This study shows that the combined gonadal hormonal therapy induces positive effects on subjective feelings of well being as compared to controls, although at baseline both groups were not sewerely clinically impaired (sleep, physical or psychological complaints etc.). Effects of HRT on memory functions were only seen when users were compared with their own baseline functioning. Hence, progestagens do not oppose the effects of estrogens on subjective feelings of well being or on memory. The findings also indicate that the use of a particular design can be essential in finding (small) effects. Studies that were performed using a cross-over design (within subjects) reported effects on memory (e.g. Sherwin, 1988) while others, which used a parallel groups design, (between subjects) did not find effects on memory (Rauramo, Lagerspetz, Engblom \& Punnonen, 1975; Vanhulle \& Demolle, 1976). However, it should be kept in mind that double blind testing is not possible (withdawal bleeding and reduction flushes) and that expectancy effects may have confounded the results.

In line with this notion are the findings of a case-control studly presented in chapter 11 . This study involved women of middle-age who were unaware of the purpose of the experiment. No positive effects on subjective scales of well being or on memory were found, although subjects with HRT were faster on sensorimotor speed tasks as compared to controls. It was concluded that the memory task may have been too difficult to reveal effects of ERT, since ERT may have a general activating, rather than a specific, effect on cognitive functions. Thus, the subjective feelings of well being in the study described in chapter $₫ 2$ may indeed have been influenced by a posittve expectancy of the drug. However, the finding, that the women investigated in chapter 11 showed a lower level of subjective well being but still were faster in information processing as compared to controls, may indicate that sensorimotor speed functions can be enhanced independently of subjective well being.

With respect to our proposal as to the use of caffeine as a golden standard drug, the results of HRT look promosing. It was stated that a new potential enhancer should be evaluated wilh caffeine and should have effects at least equal to the effects of caffeine. In the scopolamine model, one additional word on average was recalled after a delay with caffeine as compared to placebo. However, two additional words on average were recalled after a delay in women using HRT as compared to their baseline perfornance (chap. 12). As such. HRT has an effect that is stronger than caffeine and can thus be considered to be a potent cognition enhancer. To test this hypothesis, caffeine should be tested with HRT in a model which blocks the symthesis of gonadal homones (LHRH antagonist).

In sum, the findings of our studies suggest that an approach which is concerned with the maintenance of physiological systems and protect against a structural decline can be fruitful in the quest for cognition enhancers to treat age-related cognitive decline. 


\subsection{Concluding remarks}

In conclusion, age-related cognitive declite is apparent in a slowing of cognitive functions, especially on complex tasks. In addition, some of the variance of complex menory task performance is explained by subjective experienced health. As for the models used for agerelated cognitive decline, the scopolamine and mill hypoxia model hawe prowen their worth with regard to their predictive value. Hyperventilation was not a better model than hypoxia, but physical exhaustion may be used in the future when testing cognition enhancing drugs. Other models or combination of models still await evaluation. In addition, the studies of models of agerelated cognitive decline ndicate that subjectiwe experienced well-being may also interfere with the speed of information processing. However, the study with elderly women (chap. 11) on the affect of HRT showed that subjective well-being can also be lowered independent of fast information processing.

Caffeine was seen to have complex actions in the various models. This lead to the hypothesis that the interpretations of the additional arousal due to caffeine consumption may be important in the effect of caffeine on cognitive functions. Alternatively, caffeine may affect cognitive functions through its influence on seweral different neurotransmittersystems, an effect which is most apparent in case of a deficiency. The studies into the effect of caffeine have shown that the use of several different modells may be obligatory when testing a potential cognition enhancing drag. This will enable future investigators to establish the effective dosage, the possible pathways through which a drug acts on cognitive functions, and the tests to use when evaluating the drug in the target population. Our findings also suggest that middle-aged subjects may be a relevant group to use in the experimental studies of the effect of cognition enhancers.

In conclusion, this thesis has shown that several relevant methods exist which do not involve the target population for treatment with cognition enhancers (i.e. the elderly subjects with objective cognitive impairment). Models of age-related cognitive decline (scopolamine, mild lhypoxia, and physical exhaustion) can be of value in testing potential cognition enhancers. In addition, evaluation of drug effects in large cross sectional samples of otherwise healthy subjects (in this thesis the MAastricht Aging Study) may prove a relevant source of information. 


\section{References}

Adam, J.J., Teeken, J.C., Ypelaar, P.J.C., \& Verstapppen, F.T.J. (in press), Human information processing during physical exercise: manipulating mental processing demands International Journal of Sport Psychology.

Aggelton, J. (1996, 18-20th September). Dissociating neural substrates of recalli and recoginition, Paper presented at the Annual Meeting of the PSychobiology Section of the British Psychological Association, Landale: England.

Albrecht, R. F., Miletich, D. J., \& Ruttle, M. (1996). Cerebral effects of extended hyperventilation in unanesthetized goats. Stroke 18(3), 649-655.

Allain, H., Boyer, P., Kossmann, L., Lepine, J. P.n \& Kanowski, S. (1990). Therapeutic target for cognition enhancers: diagnosis and clinicall phemomenology. Phamacopswchiatry 23, 49-51.

Altman, H. J. \& Normile, H. J. (1988). What is the nature of the role of the serotonergic nervous system in learning and memory: prospects for development of an effective treatment strategy for senile dementiat Neurobiology of Aging, 9(5-6), 627-38.

Amaducci, L. A., Fratiglioni, L., Rocca, W. A., Fieschi, C., Liwrea, P., Pedone, D., Bracco, L., Lippi, A., Gandolofo, C., Bino, G., Prencipe, M., Bonatti, M. L.., Girotti, F., Carella, F., Tavolato, B., Ferla, S., Lenzi, G. L., Carolei, A., Ganbi, A. Grigoletto, F, \& Schoenberg, B. S. (1986). Risk factors for clinically diagnosed Alzheimer's disease: a case-control study of an Italian population. Neurology 36. 922 . 931.

Amaducci, $L_{n,}$ \& group (1988). Phosphatidylserine in the treatment of Alzheimer's disease: results of a multicenter study. Psychopharmacology Bulletin. 24, 130-134.

American Psychiatric Association. (1994). Diagnostic and Statistical Mamual of Mental Disorders. (4t the ed.) Washington, DC: American Psychiatric Association.

American Psychological Association. (1994). Publication Manual of the American Psychological Association. (4th ed.). Washington, DC: American Psychological Association.

Anderson, KJ., \& Revelle, W. (1994). Impulsivity and time of day: is rate of change in arousall a function of impulsivity? Journal of Personality and Social Psychology, 67(2), 334-344.

Anderson, K.J., Revelle, W., \& Lynch. M.J. (1989). Caffeine, impulsivity, and memory scanning: A comparison of two explanations. for the Yerkes-Dodson effect. Motivation and Emotion, 8, 614-624.

Antuono, P. G. (1995), Effectiveness and safety of velnacrine for the treatment of Alzheimer's disease. A doubleblind, placebo-controlled study. Mentane Study Group. Archives of Internal Medicine, 155(16), 1766-72.

Arrindell, W. A., \& Ettema, J. H. M. (1986). SCL-90. Een multidimensiomele psychopathologie indicator [SCL-90. A multidimensional indicator of psychopathology]. Lisse, "The Netherlands: Swets \& Zeitlinger.

Aylward, M. (1973). Plasma tryptophan levels and mental depression in postnenopausal subjects. Efffects of oral piperazine-oestrone sulphate. IRSC Medical Siciences, 1, 30-34.

Bäckström, T., Bixo, M. \& Hammarbä̀ck, S. (1985). Ovarian steroid hormones. Effects on nood, behavior and brain excitability. Acka Obsterricia ei Cymaecologica Scandinawica Suppl. $130,19-24$.

Baddeley, A. D. \& Hitch, G. (1974). Working memory. In G. Bower (Ed), The Pychology of Lewaning atad Motivation, (Vol. 8, pp. 47-90). New York: Academic Press.

Baddeley, A. D. (1986). Working Menory. Oxford, England: Clarendon Press.

Bahrke, M. S. \& Shukin, H. B. (1993). Efrects of altitude on mood, behavior and cogmitive functioning. A review. Sports Medicine, $16(2), 97-125$.

Balke, B. \& Lillehei, I. P. (1956). Adaptive responses to hyperventilation. Jourmal of Applied Physiglogy 9. $371-374$.

Bard, C. \& Fleury, M. (1987) Influence of imposed melabolic fatigue on visual capacity components, Pencephual and Motor Skills. 47, $1283-1287$.

Baron, J. C. \& Marchal, G. (1992). Cerebral and cardiovascular aging and brain energy metabolism. Studies with positron-enission tomography in man. Presse Med Jul. 21(26), 1231-7

Barrett-Connor, E. \& Kritz-Silverstein, D. (1993). Estrogen replacement therapy and cognitive function in older women. Journal of the American Medical Association, 269(20), 2637-41.

Bartus, R. T., Dean, R. L., Beer, B., \& Lippa, A. S. (1982). The cholinergic hypothesis of geriatric memory dysfunction. Science, $217(4558), 408-417$. 
Wattig, K., Buxzi, R., Marrin, J.R.,8 Fieierabend, J.M. (1984). The effects of caffeine on physiological functions and mental performance Experientia 40, 1218-1223.

Beatie, $c$. w. Rogers, C. H. \& Soyka, L. F. (1973). Inftuence of ovariectony and ovarian steroids on bypothalamic tyrosine hydroxylase activity in the rat Endocrinology 91, 276-286.

Benthiam, P. W. (1990). Preliminary communication. A double blind placebo-controlled trial of L-tryptophan to assess the degree of cognitive and behavioral improvement in patients with Alaheimer-type dementia and to compare differential responise in clinical sub-groups. International Clinical Psvchophamacology, 5, $261-272$.

Benton, D. \& Owens. D.S. (1993) Blood glucose and human memory. Psychopharmacology. 1:3, 83-88

Bergener, M. (1993). Treatment of cognitive disturbances in old age: methodological and clinical aspects. Clinical Neuropharmacotogy. 16 Suppl L, S46-S50.

Biaggioni, I., Paul, S., Puckett, A., \& Arzubiaga, C. (1991). Caffeine and theophylline as an adenosine receptor antagonist it humans. The Journal of Pharnacology and Experimental Therapeutics. 258, 589-593.

Billis, A. G. (1937). Blocking in mental fatigue and anoxemia compared. Journal of clinical and Experimental Psychology, 20, 437-452.

Birkhăuser, M. H. (1996, December 47). The problem of menopause. Paper presented at the 2nd International Symposium 'Women's Health and Menopause Risk Reduction Strategies', Forence (Italy), pp. 29.

Birren, J. E \& Schaie, K. W. (1990). Handbook of the Psychology of Aging (3 ed.). New York: Van Nostrand Reimhold.

Blanchard, I., Sawers, S.J.A. (1983). Comparative pharmacokinetics of caffeine in young and eldenly men. Journal of Pharmacokinedics and Biopharmacology. 11 109-126.

Blass, A. G. \& Gibson, G. E. (1979). Consequences of mild, graded hypoxia. Advances in Neurology, 26, 229253.

Blom, M. M. A. (Alzheimer's foundation) (1997,August 29). Incidence of Dementia in the Netherlands Personal communication (letter). Burnik.

Bohnen, N., Jolles, J., \& Twijnstra, A. (1992) Modification of the Stroop Color Word Test improves differences between patients with mild head-injury and matched controls. The Clinical Neuropsychologist. 6 , $178-184$.

Bohnen, N., Twijnstra, A., \& Jolles, J. (1992). Post-traumatic and cmotional symptoms in different subgroups of patients with mild head injury. Brain Injury 6, $(6), 481-487$.

Bonnet, M. H. (1986). Performance and sleepiness as a function of frequency and placement of sleep disruption. Psychophysiolagy. 23(3), 263-271.

Bonnel, M. H. (1987). Sleep restoration as a function of periodic awakening, movement, or electroencephalographic change. Sleep, 10(4), 364-373.

Bonnet, M. H. (1989). The effect of sleep fragmentation on sleep and performance in younger and older subjects. Neurabiology of Aging. $10(1), 21-5$.

Bots, M. L... Pols, H. A. P., De Jong. P. T. V. M., Grobbee, D. E., \& Hofman, A. (1996). Rotterdam Ouderen Studie [Rotterdam Aging Study]. In M. Santvoort wan M. Spanjer (Eds.), Ouder Worden Gezond en Wel LHoillhy Agingl, (pp. 16-22). Utrecht: Nederlands Instituut voor Gerontologie [Dutch Institute for Gerontology].

Boutiglioni, F. (1996, December 4-7), Qverview of HRT bencfits in postmenopausal women. Paper preserted at the 2nd International Symposium 'Women's Health and Menopause Risk Reduction Strategies". Florence (Itally).

Boxtel wan, M. P. J., Buntinx, F. Houx, P. J., Metsemakers, J., \& Jolles, J. (submitted). The relation between morbidity and cognitive performance in in normal aging population.

Brand, N. \& Jolles, J. (1985), Learning and retrieval rate of words presented auditorily and visually. The lournal of General Psychology, 122(2), 201-210.

Brand, N., \& Jolles, J. (1988). Neuropsych: Computer-assisted neuropsychological assessment. In: F.J. Maarsse, L.J.M. Mulder, W.P.B. Sjouw, \& A.E. Akkermans (Eds). Computers in psychology: Methods. Instrumentation \& Psuchodiagnostics. Lisse, the Netherlands: Swets \& Zeitlinger 1494157.

Brand van den, P. A., Goldbohm, R. A. Veer, P. van het, Volovics, A., Hermus, R. J. J, \& Sturmans, F. (1990). A large-scale prospective cohort study on diet and cancer in the Netherlands. Journal of Clinical Epidemiology, 43, 286.295.

Brandeis, D., Naylor, H., Halliday, R., Callaway, E. \& Yano, L. (1992). Scopolamine effects on visual information processing, attention, and event-rellated potential map latencies. Bsychophysiology.29(3), 31536. 
Breier, A., \& Buchanan, R. W. (1992). The effects of metabolic stress on plasma progestrone in healthy volunteers and schizophrenic patients. Life Sciences, 51(9), 1527-1534.

Breier, A., Davis, O. R., Buchanan, R. W. Moricle, L. A. Munson, R. C. (1993), Effects of metabolic perturbation on plasma homovanillic acid in schizophrenia. Relationship to prafrontat cortex volume. Archives of General Psychiatry. Jul: $50(7), 541-550$.

Brenner, D. E., Kukull, W. A., Stergachis, A., wan Belle, G., Bowen, J. D., MoCormiek, W. C., Teri, L., \& Larson, E. B. (1994). Postmenopausal estrogen replacement therapy and the risk of Alzheimer's disense: a population based case-control study. American Journal of Epidenoiology 140(3), 262-267.

Briley, M. (1990). Biochemical strategies in the search for cognition enhancers. Plamrmacopsychializy, 23, 75-80.

Broe, G. A., Henderson, A. S., Creasey, H., MoCusker, E., Korten, A. E., Jorm, A. F., Longley, W. \& Anthony. J. C. (1990). A case-control study of Alzheimer's disease in Australia. Neurology 40 (November); 1698-1707.

Brouns F (1989) Effect of carbohydrate intake during warming up on the regulation of blood glucose during exercise. International Journal of Sports Medicine, 10, S68-S75

Bryan, J., \& Luszcz, M. A. (1996). Speed of information processing as a mediator between age and free-recall performance, Psychology and Aging, 11(1), 3-9.

Bryson, H. M., \& Benfield, P. (1997). Donepezil. Drugs and Aging, 10(3), 234-239.

Bungay, G. T., Vessey, M. P., \& McPherson, C. K. (1980). Study of symptoms in middle life with special reference to the menopause. British Medical Journal. Jul 12: 281(6234), 181-183.

Burger, H.G. (1996). The endocrinology of the menopause. Maturitas, March 23 (2), 129-136.

Caldwell, B. M., \& Watson, R. I. (1952). Evaluation of psychologic effects of sex hormone administration in aged women: results of therapy after 6 months. Journal of Gerontology $7,228-244$.

Callaway, E. (1984). Human information-processing; some effects of methylphenidate, age, and scopolamine. Biological Psychiatry, 19(5), 649-62.

Campbell ${ }_{n}$ S. Whitehead, M. (1977). Oestrogen therapy and the menopausal syndrome. Clinical Obstetrics and Gynaccology. $4(1), 31-47$.

Carlson, M. C., Hasher, L., Connely, S. L., Zacks, R. T. (1995). Aging, distraction, and the benefits of predictable location. Psychology and Aging. 10(3), 427-436.

Carskadon, M. A. \& Dement, W. C. (1985). Sleep loss in elderly volunteers. Sleep. 8, 207-221.

Cavaletti, G. \& Tredici, G. (1993). Long-lasting neuropsychological changes after a single high altitude climb. Acta Neurologica Scandimavia, 87, 1.03-105.

Centraal Bureau voor Statistiek-CBS (1985). Beroepenclassificatie 1984 (2nd edition) Voorburg: CBS.

Centraal Bureau voor Statistiek - CBS (1997). Bevolkingsprognose voor Nederland 1996-2050. Den Hag: SDUuitgeverij

Cerella, J., Poon, L. W., \& Williams, D. M. (1980). Age and the complexity hypothesis. In L. W. Poon (Ed.), Aging in the 1980s, (pp. 332-340). Washington DC: American Psychological Association.

Chen, M.F. Chiu, T. H., \& Lee, E. H. Y. (1992). Noradrenergic mediation of the memory-enthancing effect of corticotropin-releasing factor in the locus cereleus of rats. Psychoneuroendocinology $17(2 / 3), 113-124$.

Christensen, H. \& Birrell, P. (1991). Explicit and implicit memory in dementia and normal ageing. Psychological Research. 53, 149\%161.

Christie, J. E., Schering, A.. Ferguson, J., \& Gler, A. 1. M. (1981). Physostigmine and arecoline: effects of intravenous infusions in Alzheimer's presenile dementiat British Journal of Psychiatry. 138, 46.50.

Christie, J., Hunter, R., Bennie, J., Wilson, H., Carroll, S., \& Fink, G. (1990). Reduced plasma oestrogen stimulated neurophysin and delayed response to oestrogen challenge in Alzheimer's disease. Psychological Medicine. 20, 773-777.

Coleman, P. D., \& Flood, D. G. (1987). Neuron number and dendritic extent in normal aging and Alaheimer's disease. Neurobiology of Aging, 8, $521-545$.

Commisaris, C. J. A. M., Ponds, R. W. H. M., \& Jolles, J. (1995). Subjective memory problems and people"s need for imtervention. In: J. Jolles, P. J. Houx, M.P.J. van Boxtel, \& R. W. H. M. Ponds (Eds). Magstright Aging Study: Determinants of Cognitive Aging. Maastricht, The Netherlands: Neuropsych Publishers (pp. 79-94).

Cooper, J. R., Bloom, F. E., \& Roth, R. H. (1991). The biochemical basis of behavior. (6th ed.). Now York: Oxford University Press, p.277.

Coper, H. \& Kanowski, S. (1983). Nootropika Grundlagen und Therapie. In G. Langer \& H. Heimann (Eds) Pspchopharmaka. Grundlagen und Therapie. Vienna/New York: Springer, p.409 
Costall, B., Barnes, J. M., Hamon; M. Muller, W. E., \& Briley, M. (1990). Bidchemical models for cognition

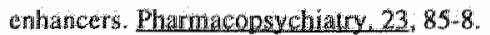

Costill, D.L., Dalsky, G.P. \& Fink, W.J. (1978). Effects of caffeine ingestion on metabolism and exercise performance. Medicine and Science in Sports, 10,155-8

Côte, J., Salmela, J. H. : Papathantsopoulu, KP. (1992) Effects of progressive exercise on attentional focus. Berceptual and Motor skills. $75,351-354$.

Coull, J. T. (1994). Pharmacological manipulations of the alpha 2-noradrenergic system. Effects on cognition. Lrugg and Aging, $5(2), 1626$.

Coull, J. T., Middleton, H. C, Robbins, T". W., Sahakian, B. J. (1995a). Clonidine and diazepam have differential effects on tests of attention and learning. Pychophamacology $120(3), 322-332$.

Coull, J. T., Middleton, H. C, Robbins, T. W. \& Sahakian, B. J. (1995b). Contrasting effects of clonidine and diazeparn on tests of working memory and planning. Psychopharmacologe 120(3), 311.321.

Coull, J. T., Sahakian, B. J, Middleton, H. C., Young, A. H., Park, S. B., McShane, R. H., Cowen, P. J., \& Robbins, T. W. (1995c). Differential effects of clonidine, haloperidol; diazepam and tryptophan depletion on focused atention and attentional search. Psychopharmacology. 121(2), 222-230.

Coyle, J. T., Price, D. L., DeLong, M. R. (1983). Alzheimer's disease: a disonder of cortical cholinergic innervation. Science 219, 1184-1190.

Coyle E, Coggan A (1984) Effectiveness of Carbohydrate Feeding in Delaying Fatigue during Prolonged Exercise. Sports Medicine, 1, 446-458

Craik F. I. M. \& Bird, M. (1982). Aging and cognitive deficits: the role of attentional resources. In F. I. M. Craik, \& S. Trehub (Ed), Aging and Cognitive Processes, (pp. 191-211). New York: Plenum.

Craik , F. I. M. (1986). A functional account of age differences in memory. In F. Klix, \& H. Hagendorf (Ed.), Human Memory and Cognitive Capabilities, (pp. 409 422). Amsterdam, Noord Holland: Elseviers Science Publishers BV.

Croisile, B., Trillet, M., Fondarai, J., Laurent, B., Mauguite, F., \& Billardon, M. (1993). Long term and highdose piracetam treatment of Alzheimer's disease. Neurology 43, 301-305.

Crook, T., Petrie, W., Wells, C., \& Massari, D. C. (1988). Effects of phospatidylserine on Alzheimer's disease. Psychopharmacology Bulletin, 21, 61-66.

Crook, T., Bartus, R. T., Ferris, S. H., Whitehouse, P., Cohen, G. D., \& Gershon, S. (1986). Age-associated memory impairment: Proposed diagnostic criteria and measures of clinical change: Report of a National Institute of Mental Health work group. Developmental Neuropsychology, 2(4), 261-276.

Crow, T.J. \& Kelman, G.R. (1973). Psychological effects of mild acute hypoxia. British Journal of Anacsthesia. 45 (4), 335-337.

Crow, T. J., \& Kelman, G. R. (1971). Effects of mild acute hypoxia on human short term memory. British Journal of Amuesthesia. 43 $(6), 548-552$.

Curran, H. V., \& Birch, B. (1991). Differentiating the sedative, psychomotor and amnesic effects of benzodiazopines: a study with midazolam and the benzodiazepine antagonist, flumazenil. Psychopharmicology Berl 103(4), 519-23.

Cutter, N. R., Haxby, J., Kay, A. D.. \& al., e. (1985). Evaluation of zimeldine in Alzheimer"s disease. Archives of Neurology. 42, 744.748.

Danjou, P., Hamon, M., Lacomblez, L., Warot, D., de al.. e. (1990). Psyclromotor, subjective and neuroendocrine effects of acute tryptophan depletion in the healthy volunteer. Psychiatry and Psychobiology. $5(1), 31-38$.

Das, A. \& Chaudhuri. S. K. (1995). Effects of sex steroids on the concentrations of some brain neurotransmitters in male and female rats: some new observations. Indian Journal of Phusiological Bharmacology, 39(3), 223-230.

Davies P. \& Maloney, A. F. J. (1976). Selective loss of central cholinergic neurons in Alzheimer"s disease. The Lancet. II. 1403.

Davinson, D., \& Parrot, A C. (1.997). Ecstasy (MDMA) in recreational users: self-reported psychological and physiological effects. Human Psychopharmacology, 12, 211-226.

Davis, J. N., Giron, L. T., Stanton, E, \& Maury, W. (1979). The effect of hypoxia on brain neurotransmitter systems. Advances in Neurology. 26, 219-224.

Davis, J. M., \& Bailey, S. P. (1997). Possible mechanisms of central nerwous system fatigue during exercise. Medicine and Science in Sports and Exercercise 29(1), $45-57$. 


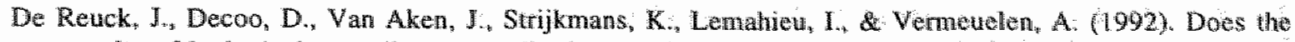
severity of leukariosis contribute to senile denentia? A comparative computeried and position entussion tomographic study. Clinical Newology and Nentrosurgery, 94(2), 113-8.

De Jonge, M. C., Traber, J. (1993). Nimodipine: Cognition; Aging and Degeneration. Chinem Neuropharmacology, 16 Suppl 1 , 25-30.

Deberdu, W. (1994). Interaction between psychological and pharmacological treatment in cognitive impairment. Life Sciences $55(25-26), 2057-66$

Deelman, B. G., Brouwer, W. H., Zomeren, A. H. V. \& Saan, R. J. (1980). Functiestoormissen nia tramma capitis, In A. Jennekens-Schinkel, J. J. Diamant, H. $\mathbb{F}$. A. Diesfeldt, \&. R. Hanma (Bds), Neuropsychologie in Nederland. Deventer, The Netherlands: Van Loghum Slaterus.

Dehlin, O., Hedenrud, B., Jansson, P., N Norgärd, J. (1985). A double-blind comparison of alaprochate and placebo in the treatment of patients with senile dementia. Acta Psychiatrica Scandinavica. 21. 190-196.

Delacour, J., Bassant, M. H. Onofry, M., Santucci, V., \& Kleinlogel, H. (1990). Electrophysiological models for the study of cognition enhancers. Pharmacopsychiary, 23,90-3.

Delgado, P. L., Miller, H. L., Salomon, R. M., Licinio, J., Heninger, G. R., Gelenberg, A. I., E Chatrmey, D. S. (1993). Monoamines and the mechanism of antidepressant action: effects of catcelwolanime depletion on mood of patients treated with antidepressants. Psychopharmacology Bull, 29(3), 389-96.

Delignières, D., Brisswalter, J., \& Legros, P. (1994) Influence of plyysical exercise on choice reaction time in sports experts: the mediating rolle of resounce allocation. Journal of Human Movement Sudios, $27,173 \% 188$.

Denison, D. M., Ledwith, F., \& Pulton, E. C. (1966). Complex reaction times at simulated cabin altitudes of 5000 feet and 8000 feet. Aerospace Medicine, 37, 1010-1013.

Dernerstein, L. Burrows, G. D., Hyman, G., \& Sharpe, K. (1979). Homone therapy and affect. Maturitas 1. 247-254.

Dennerstein, L. (1987). Psychological changes, In D. R. Mishell (Ed.), Monophase Physiology and Pharmacology, (pp. 15-127). Chicago: Year Book Medical Publishers Inc.

Desmond, T. W., Tatemichi, T. K., Paik, M. \& Stern, Y. (1993). Risk factors for cerebrovascular disease as of body fat, plasma lipoproteins, and cardiovascular disease. Archives of Neurology, 50, 1.62-166.

Dijkstra, J. (1997). An Operation under General Angesthesia and Cognitive Ageing, Maastricht: Neuropsych Publishers.

Directiorat-Generaal woor arbeids voorzieningen (1989). Handleiding voor de functic-analyse (Matual for job analysis) SDU Uitgeverij: Den Haag.

Dirken. J. M. (1967). Het meten van "stress" in industrièle situatios IMeasurement of "stress' in industrial situations]. Groningen: Wolters.

Ditkoff, E. C., Crary, W. G., Cristo, M., Lobo, R. A. (1991). Estrogen improves psychological function in asymptomatic postmenopausal women. Obstetrics and Gynecology, 78(6), 991-5.

Doraiswamy, P. M., Bieber, F., Kaiser, L., Krishnan, K. R., Reuning-Schrerer, J., \& Gulanski, B. (1997). The Alylteimer's disease assessment scale: patterns and predictors of baseline cognitive performance in multicenter Alzheimer's discase trails. Neurology 48(6). 1511-1517.

Drachman, D. A. (1977). Memory and cognitive function in man: does the cholinergic system have a specific role ? Nehrology, 27, 783.790 .

Duka $a_{n}$, On, H, Rohloff, A, \& Voet, B. (1996). The effects of a benzodiazepine receptor antagonist betacarboline ZK-93426 on scopolamine-induced impairment on attention, memory and psychonoton skills. Psychopharmacology Berl $123(4), 361-73$.

Duker, H. (1957). Leistungsfähigkei und Keimdrisenhormone. (Vol, 140). München: Bartl.

Dukes, M. N. G, \& Dijke, C. P. H. v. (1984). Bijwerkingen van genesmiddelon. (lst ed.). Amsterdam: Elsevier.

Dukes, M. N. G. (1988). Meyler's side effects of drugs-an encychopdia ol adverse reactions and inlcetactions. (11 ed.). Amsterdam: Elsevier.

Earles, J. L.. Salthouse, T. A. (1995), Interrelations of age, health, and speed Jourmal of Gieconology: Psychological Sciences, $50 \mathrm{~B}$, P33-P41.

Earles, J. L., Connor, L. T., Frieske, D. Park, D., Smith, A. D., \& Zwahr, M. (1997). Age differences in inhibition: possible causes and corsequences. Aging. Neuropsychology. and Cognition, 4(1), 45-57.

Easterbrook. J.A. (1959) The effects of emotion on cue utilization and the organization of bethavior. Psychological Review, 66, 183-281.

Ellingsen, I., Hauge A., Nicolaysen, G, Thiresen, M., \& Walloe, L. (1987). Changes in human cerebral blood flow due to step changes in $\mathrm{paCO}_{2}$ and $\mathrm{paO}_{2}$. Acta Psychologica Sicandinavica. 129, 495-502. 
ENis, C. M. Lemmeris, G., \& Parkes, J. D. (1996). Melatonin and insomnia. Ioumal of Sleep Research. March: $5(1), 61-65$.

Erikson GC. Hager LB, Houseworth C. Dungan J, Petros T, Beckwith BE (1985) The effects of caffeine on memory for word lists. Physiology and Behavior, $35: 47-51$

Erinting. J. (1978). The 10h Amual Harry G. Armstrong Lecture: prevention of hypoxia. Aviation, Space, and Environmentil Medicine. 49, 495502.

Essig D, Costill D, Van Handel P (1980) Effects of caffeine ingestion on unilization of muscle glyeogen and lipid dering ergometer eycling. Interbational Joumal of Sports Medicine 1 86-90

Fahle, M. o Daum, I. (in preparation). Visual learning and memory as functions of age.

Fastenau, P. S., Dentburg, N. 1., \& Abeles, N. (1996). Age differences in retrieval: further support for the resources-reduction thypothesis. Bsychology and Aging $11(1), 140-146$.

Fedor-Freyberg, P. (1977). The influence of estrogens on the well-being and mental performance on the climacteric and postmenopausal women. Acta Obstetricia ef Gynaecologica Scandinavia, 64, 1220 .

Feinberg, I, \& Floyd T. C. (1982). The regulation of thuman sleep. Humam Neurobiology .11, 185-194.

Fillit, H., Weinreb, H., Cholsi, I, Luine, V, McEwen, B., Amador, R., \& Zabriskie, J. (1986a). Observations in a preliminary open trial of estradiol therapy for senile dementia-Alzheimer's type. Pexclosineuroendocrinology $11.337-345$.

Fillet, H. M., Ashby, D., Weinreb, H. Zabriskie, J. B., Luine, V. N., McEwen, B. S. (1986b). Estrogen lewels in postmenopausal women with senile dementia-Alzheimer's type (SDAT) are significantly lower than matched controls. Annual Meeting of the Society for Neuroscience. 12. 259.11.

Fillit, H. (1994). Estrogens in the Pathogenes is and 'Treatment of Alzheimer's Disease in Postmenopausal Wornen. Annual meeting of the New York Academy of Sciences, 743, 233-239.

Fioravanti, M., Bergamasco, B., Bocola, V., Martucci, N. Nappi, G., Neri, G., Fabbrimi, G., \& Agnoli, A. (1991). A multicentre, double-blind, controlled study of piracetam vs placebo in geriatric patients with nonvascular mildwmoderate impairment in cognition. New Trends in Clinical Neuropharmacology, V(1), 2734.

Fischoff, P. K., Saletu, B., Rüther, E., Litschauer, G., Möslinger-Gehmayr, R., \&e Hermann, W. M. (1992). Therapeutic Efficacy of Pyritinol in Patients with Senile Dementia of the Alzheimer Type (SDAT) and Multi-Infarct Dementia (MID). Neuropsychobiology, 26, 65-70.

Fisk. J. E. We Warr, P. (1996). Age and working memory: the role of perceptual speed, the central executive, and the phonological loop. Psychology and Aging. $11(2), 316-323$.

Fleury, M.s Bard, C., Jobin, J., \& Carriere, L. (1981) Influence of different types of physical fatigue on a visual detection task. Perceptual and Motor Skills, 53(3), 723-730.

Fleury, M. Bard, C., \& Carriere, L. (1981) Effects of physical or perceptual work loads on a coincidence/anticipation task. Perceptual and Molor Skills, 53(3), 843-850.

Flint Beal, M. (1992). A decrease of metabolism in parietal and temporal lobes in presenile DAT. Annals of Nurology. 31, 1194130.

Folkard, S., Arendt, J, \& Clark, M. (1993). Can melattonin improve shift worker's tolerance of the night shift? Some preliminary findings. Chromobjology Jaternational, Octi 10(5), 315-320.

Foreman, N., Barraclough, S., Morre, C., Mehta. A. \& Madon, M. (1989) High doses of caffeine impair performance on a mumerical version of the Stroop task in men. Pharmacology. Biochemistry and Behavior. $32,399-403$

Forester, P. J. G. (1985). Effect of different ascent profiles on performance at $4200 \mathrm{~m}$, elevation. Aviation. Space, ind Environmental Medicine $56,758-764$.

Fowler, B., Paul, M., Porlier, G., Elcombe D. D., \& Taylor, M. (1985). A re-evaluation of the minimum allitude at which hypoxic performance decrements can be detected. Ergonomics, 28(5), 781-791.

Fowler, B., Taylor, M. \& Porlier, G. (1987). The effects of hypoxia on reaction time and movement time components of a perceptual-motor lask. Ergonomics, 30(10), 1475-1485.

Fowler, B., Prlic, H., B Brabant, M. (1994). Acute hypoxia fails to influence two aspects of short term memory: implications for the source of cognitive deficis. Aviation. Space and Environmental Medicine, $65(7), 641-645$.

Fozard, J. L., Vercryssen, M., Reynolds, S. L.. Hancock, P. A. \& Quilter, R. E. (1994). Age differences and changes in reaction time: the Baltimore longitudinal study of aging. Journal of Gerontology: Psychological Sciencos 49(4), 179-189. 
Frith, C. D. Richardson, J. T. E, Samuel, M, Crow, T. I., M McKema, P. 3. (1984). The offects of intravenous diazepan and byoscine upon human memory. Quarterly Journal of Experimental PSychology Human Experimental Pychology, 36, (1), 133-144.

Funk, I. L., Mortel, K. F. \& Meyer, J. S. (1991). Effects of estrogen replacement therapy on cetebral perthsion and cognition among postmenopausal women. Dementia $2(5), 268-272$.

Gamzu, E. R., Birkhimer, L. J., Hoover, T., G Gracon, S. T. (1990). Early human triats in the assessment of cognition activators. Pharmacopsychiatry, 23, 44-8.

Gamzu, E.R., Birkhimer, L.J., Hoover T. \& Gracon, S.T. (1990).Clinical testing of cognition enhancers: prediction of efficacy. Phammacopspchiatry, 23,57.59

Garssen, B. (1986). Psychofysiologie van de ademhaling en hethypenentiatisyndroom. Delft Eburon.

Gierard, L., Zacks, R., Hasher, L.. \& Radvansky G. A (1991). Age deficits in retrieval: the far effect. Iounals of Gerontalogy: Psvehological Sciences Jul 46(4), PH $31-\mathrm{Pl} 36$.

Ghomeim, M. M., Hinrichs, J. V. Chiang, C. K. Loke, W. H. (1986). Pharnacokinetics pharmacodynamic interactions berween caffeine and diazepam. Journal of Clinica Psychophamocalogy $6(2)$. $75=80$.

Ghoneim, M. M. Block. R. I., Ping, S. T., Eahaby, H. M., Hinrichs, J. V. (1993). The interactions of midazolam and flumazenil on human memory and cognition. Anesthesiologe 29(6), 1183-92.

Ghoneim, M. M., \& Mewaldt, S. P. (1977). Studies on buman memory: the interactions of diazepim, scopolamine, and physostigmine. Psychopharmatology Berl.52(1), 1-6.

Gibbs, R. B. (1994). Estrogen and nerve growth factor-related systems in brain. Annals of the New Xork Academy of Sciences, $14(743), 165-199$.

Gibson, G. E., Pulsinelli, W., Blass, J. P., Duffy, T. E. (198j). Brain dysfunction in mild to moderate hypoxia. American Journal of Medicine $70,1247 \times 1254$.

Gibson, T. M. (1978). Elfects of hypocapnia on psychomotor and iniellectuall pertiormance. Avidton. Sytace, and Environmental Medicine.49, 943-946.

Gitelman, D.R. \& Prohovnik. I. (1992). Muscarinic and nicotinic contributions to cogntive functions and cortical blood How. Neurobiology and Aging, 13(2), 313-318

Godsland, I. F., Wynn, V., Path, F. R. C " Crook, D., \& Miller, N. Fi, (1987). Sex, plasma lipoproteins, and atherosclerosis: prevailing assumptions and outstanding questions. Amarican Hant Jomon, 114(6), 1467. 1503.

Goebel, J. A., Birge, S. J., Price, S. C. Hanson, J. M., \& Fishel, D. G. (1995), Estrogen replacement therapy and postural stability in the elderly. Americian Journal of Otology. 16(4); 470-474.

Gold A. E. Deary, I. J, MacLeod, K. M., Thomson, K. J., \& Frier, B. M. (1995), Cognive funciion during insulin-induced hypoglycemia in humans: short-term cerebral adaptation does not occur. Psychoptarmacology Berl, 19(3), 325-33.

Goldman-Rakic, P. S. (1996). Regional and cellulat fractionation of working memory. Proceedings of the National Academy of Sciences of the U, S. $93(24), 13473-13780$.

Goldstein, A. \& Kaizer, S. (1969). Psychotropic eftects of caffeine in man, II. A questionnaile survey of coffee drinking and its effects on group of housewives. Clinical Plawmigology Theropy 19 . 477.488

Goodman, Y., Bruce, A. J. Cheng, B., \& Mattson, M. P. (1996). Estrogens attenuate and corticosterone exacerbates excitotoxity, ox dative injury, and amyloid beptide toxicity in hippocampal netrons. Jourmal ol Neurochemisty, $66(5), 1836-1844$

Goodnick, P. \& Gershon, S. (1984). Chemotherapy of cognitive disorders in geriatric subjects. Lownallof Clinical Psychiatry. 45(5), 196-209.

Gorenstein, C., Bernik, M. A., Pompéia, S., \& Marcourakis, T. (1995). Impairment of performance associalod with long-term use of benzodiazepines. Jourmal of Psychopharmacology, 9(4), 3-3-318.

Gottries, C. G., Adolfsson, R., Aquilonius, S. M., Carlsson, A., Eckemas, S.-A., Mordberg. A., Oreland, I., Svennerholm, L., Winblad, A., \& Wilberg, A. (1983). Biochernical changes in dementix disorders of the Alaheimer type. Neurobiology of Aging, 4, $261-271$.

Gottfries, C. G., Bartfai, T., Carlsson, A., Eckermas, S.-A., Svennerhohm, L. (1986). Multiple deficits in both gray and white matter in Alzheimer's brains. Progress in Neuropsychophatuacology 8 Bulogical Psychiaury $10,405 \times 413$.

Gould, E., Woolly, C. S., Frankfurt, M. \& McEwen, B. S. (1990). Gonadal steroids regulate dendritic spine density in hippocampus pyramidal cells in adulthood. Journal of Neuroscience 12, 1286-1291.

Graves-Borenstein, A., White, E. Koepsell, T. D., Reifler, B. V., van Belle, G, Larson, E. B., \& Raskind, M. (1990). A case-control study of Alzheimer's disease. Annal of Neurology.28, $766-774$. 
Griffuths; F. Convery. B. (1995): Women's use of homone replacement therapy for relief of menopausal symptoms, fior prewention of ustiteoporosis; and after hysterectomy. Br J Gen Praft. 45(396), 355-8.

Grobe-Einsler, R. (1993). Clinical aspects of nimodipine. Clinical Neuropharmacology 16, \$39-\$45.

Grosss, F. Bysin, F. (1996). Phototherapy in psychiatry" clinical update and review of indications. Encephale. March-April 22(2), 143-148.

Gunter, 1. C., Van der Zande, R. D. Wiethoff, M., Mulder, G., \& Mulder, L. J. M. (1987). Visual selecive atterition during theaningful noise and after sleep deprivation. In $\mathrm{R}$ Johnson, Rohrbach, J.W. Paramuanan; R. (Ed.), Cumrent Trends in Event-Related Romential Research, (pp. 99-108). Amsterdam : Elsewicr Science Publishers B.V.

Gutin, B. (1973). Exercise-Induced Activation and Human Performance: A Review. Research Quarterly, 44, 256268.

Guyton, A.C. (1991). Aviation, high altitude, and space physiology. In: A.C. Guyton (Ed.) Texthook off Medical Physiology. (pp. 77-102) London: Prentice-Hall International Ine.

Hackman, B. W, \& Galbraith, D. (1976). Replacement therapy and piperazine oestrone sulphate ("Harmogen') and its effect on memory. Current Medical Research Opinions, 4(4), 303-306.

Hackman, B. W., \& Gatbrath, D. (1977). Six month study of oestrogen therapy with piperazine oestrone sulphate and its effects on memory. Current Medical Research Opinions, 4(\$3), 21-27.

Haimov, I., Lavie, P., Laudon, M., Herer, P., Vigder, C. \& Zisapel, N. (1995). Melatonin replacement therapy of elderly insomniacs. Sleep. Sep: 18(7), 598-603.

Halbreich, U., Rojansky, N., Palter, S., Tworek, H., Hissin, P., \& Wang, K. (1995b). Estrogen augments serotonergic activity in postmenopausial women. Biological Psychiatry $37(7), 434-441$.

Halbreich, U. (1997). Role of estrogen in postmenopausal depression. Neurology, 48(Suppl 7), S16-S20.

Halldane, J. S., Kellas, A. M., \& Kennaway, E. L. (1919). Experiments on acelimatization to reduced atmospheric pressure. Iounal of Physiology 53, 183-206.

Hall, S. T., Puech, A., Schaffler, K., Wesnes, K. \& Gamzu, E. R. (1990). Early elinical testing of cognition enhancers. prediction of efficacy. Bharmacopsychiatry, 23, 57-8.

Hancock. S. McNaughtoth, L. (1986) Effects of fatigue on ability to process visual information by experienced orienteers. Perceptual and Motor Skills 62,491-498.

Hartesweldt van, C. \& Joyce, J. N. (1986). Effects of estrogen on the basal ganglia. Neuroscience and Biobehavioral Reviews. 10, 1.14.

Hasher, L., Z Zacks, R. T. (1979). Automatic and effortful processes in memory. Ilournal of Experimental Bsycholiogy: General, 108(3), 356-388.

Hasher, L., \& Zacks, R. T. (1988). Working memory, comprehension, and aging: a review and a new view. In G. Bower (Ed).), The Psychology of Learning and Motivation, (Vol. 22, pp. 193-226). New York: Academic Press.

Henderson, V. W., Paganini Hill, A. Emanuel, C. K., Dunn, M. E., \& Buckwalter, J. (1994). Estrogen replacement therapy in older women: Comparisons between Alzheimer's disease cases and nondemented. control subjects. Archivas ol Nefurology. 51 (9), 896.900.

Henderson, V. W. \& Buckwalter, J G. (1994). Cognitive deficits of men and women with Alzheimer's disease. Nevicology, $44,90-96$.

Henderson. V. W., Watı, L., \& Buckwalter, J. G. (1996). Cognitive skills associated with estrogen replacement therapy in wonien with Alaheimer's disease. Psychoneuroendocrinology, 21(4), 421-430.

Heyman, A., Wilkinson, W. E., Stafford, J. A. Helms, M. J., Sigmon, A. H., \& Weinberg, T. (1984). Alzheimer's disease: a study of epidemiological aspects. Annals of Neurology. 15, 335-341.

Hiemke, C., Banger, M. Kohsik, R., Hundr, M., \& Ghraf, R. (1992). Actions of sex hormones on the brain. Progress in Neurophamacology \& Biological Psychiatry, 16, 377-388.

Higgins, M.J., Hosseinzadeh, H., MacGregor, D.G., Oglivy, H., \& Stone, T.W. (1994). Release and actions of adenosine in the central nervous system. Pharmacy World \& Science 16(2), 62-68.

Hindmarch. I. \& Tiplady, B. (1994). A comparison of the psychometric effects of remoxipride with those of haloperidol, thioridazine, and lorazepam in healthy wolunteers. Human Psychopharmacology Clinical and Experimental. 9(1), 43-49.

Hogervorst, E., Riedel, W. J., Rozendaal, N ${ }_{w}$ Van Boxtel, M. P. J., \& Jolles, J. (1995). Smoking and cognitive complaints. In J. Jolles, P. J. Houx, M. P. J. Van Boxtel, \& R. W. H. M. Ponds (Eds.), Magastrich Aging Study: Determinants of Cognitive Aging, (pp. 105-110). Maastricht, The Netherlands: Neuropsych Publishers. 
Hogervorst, $E_{\text {, }}$ Riedel, W. J., Jeukendrup, A. \& Jolles, J. (1996). Cogative performance after strentous exercise. Percepulal and Motor Skils, 83(2), 479-488.

Hogervorst, E. Riedel, W. J., wan Boxtel, M.P.J. \& Jolles, J. (In preparation). Hormonal Replacement Therapy and cognitive perfornance in middle-aged women: results from a large eross sectional study.

Hogervorst, E., Riedel, W., Hameleers, P., Seij, S., Van Boxiel, M., Visser, P., \&olles, J. (submitted-a). Voluntary hypocapnic Hyperventilation and Hypoxia as Models for Cognitive Deficiency.

Hogervorst, E., Riedel, W., Hameleers, P., Sey, S., Rozendal, N., Van Boxtel, M., Visser, P. Schmitt, J., Jolles, J. (submitted-b), Caffeine decreases cognitive task performance in the hypoxia and the hyperventilation model.

Hogervorst, E., Riedel, W. J., Houx, P. J., Schmitt, J. A. I., \& Jolles, J. (submitted-c). The effects of age, health, and speed of information processing on performance of a visual learning task with distraction. Azing. Neuropsychology and Cognition.

Hogervorst, E., Riedel, W. J., Kovacs, E., Brouns, F., \& Jolles, J. (submitted-d). Caffeine in Carbohydrate Electrolyte Solutions Improves Cognitive Performance Before and After Strenuous Physical Exercise

Hogervorst, E., Riedel, W., Schmit, J., \& Jolles, J. (accepted). Caffeine improves memory performance during distraction in middle aged, but not in young or old subjects. Luman Psychopharmacology.

Holford, N. H., \& Peace, K. (1994). The effect of tacrine and lecithin in Alzheimer's disease. A population pharmacodynamic analysis of five clinical trials. European foumal of Clinical Pharmacology 47(1), 17-23.

Hollister, L. E (1990). Problems in the search for cognition enhancers. Pharmacopsychiatry 23, 33-6.

Holzbauer, M. \& Yondin, M. B. H. (1973). The oestrus cycle and monamine oxidase activity. British Journal of Pharmacology, 48, 600-608.

Honmer, D., Weingartner, H. J., \& Breier, A. (1993). Dissociation of benzodiazepine-induced amnesia from sedation by flumazenil pretreatment. Psychopharmacology. 112( 4 ), 455-450.

Honer, W. G., Probovnik, I., Smith, G., \& Lucas, L. R. (1988). Scopolamine reduces frontal cortex perfusion. Journal of Cerebral Blood Flow and Metabolism, 8, 635-641.

Honjo, H., Ogino, Y., Naitoh, K., Urabe, M., Kitawaki, J., Yashuda, J., Yamamoto, T., Ishihara, S., Okada, H., Yonezawa, T., Hayashi, K., \& Nambara, T. (1989). In vivo effects of estrone sulfate on the central nervous system-senile dementia (Alzheimer's type). J Steroid Biochem, 34, 521-525.

Honjo, H., Ogino, Y., Tanaka, K., et al., (1993). An effect of conjugated estrogen to cognitive impairment in women with senile dementia-Alzheimer's type: a placebo-controlled double blind study. Iournal of the Japanese Menopause Society 1, 167-171.

Honjo, H., Tanaka, T., Urabe, M. Okada, H., Hayashi, M., \& Hayashi, K. (1995). Senile dementiaAlzheimer's type and estrogen. Hormone and Metabolic Research, 27, 204-207.

Home, J. (1992). Human slow wave sleep: a review and appraisal of recent findings, with implications for sleep functions, and psychiatric illness. Experientia, 48(10), 941-54.

Hout, van den M.A., de Jong, P., Zandbergen, J., \& Merckelbach, H. (1990). Waning of paric sensations during prolonged hyperventilation. Behavioral Research and Therapy_28 (5), 445-448.

Houx, P. J., Vreeling. F. W. Jolles, J. (1989). Cognitiye aging and risk factors for dementia, Paper presented at the Alzheimer's Disease: Proceedings of the 5th meeting of the international study group on the Pharmacology of Memory disorders associated with aging.

Houx, P. J., Vreeling, F, W, \& Jolles, J (1990). Risk factors for age-associated cognitive decline. In $C$. F. A. Van Bezooijen, R. Ravid, \& A. A. J. Verhofstad (Eds.), From Gene io Man, (pp. 190-193). The Hague, The Netherlands: Pasmans.

Houx, P. J., Vreeling, F. W., \& Jolles, J. (1991). Rigorous health screening reduces age effect on memory scamning task. Brain and Cognition, $15(2), 246-60$.

Houx, P. I., Vreeling, F. W., \& Jolles, J. (1991). Age-associated cognitive decline is related to biological life events. In K. Iqbal, D. R. C. McLachlan, B. Winblad, \& H. M. Wisniewski (Eds.), Alzheinner's Disease: Basic Mechanisms. Diagnosis and Therapeulic Strategies, (pp. 353-359). Chichester, UK: John Wiley \& Sons Lid.

Houx, P. J., Vreeling, F. W., \& Jolles, J. (1993), Stroop interference: aging effects assessed with the Stroop Color-Word Test. Experimental Aging Research, 19, 209-224.

Houx, P. I. \& Jolles, J. (1993y. Age-related decline of psychomotor speed: effects of age, brain thealth, sex, and education. Perceptual and Motor Skills, 76, 195-211.

Houx, P. J. \& Jolles, J. (1994). Vulnerability factors for age-related cognitive decline. In R. L. Isatacson, \& K.F. Jensen (Ed.). The Vulnerabje Brain and Environmental Risks, (Vol. 3: Toxins in Air and Water, pp 25-41). New York: Plenum Press. 
Hoyer, 5. (1992), Oxidative energy metabolism in Alzheimer's brain. Molecular and Chemical Neuropathology. 16. 207.

Huppert, F. A (1982). Memory impaiment associated with chronic hypoxia in man. Thorax, 37, 858-860.

Huppert, F. A. (1991). Age-refated changes in memory: leaming and remembering new information. In $F$. Boller \& J. Grafman (Eds), Handbook of Neuropsychology. (Vol. 5, pp. 123-147). Amsterdam: Elsevier Science Publishers BV.

Isacs, L, \& Pohlman, R (1991). Effects of exercise intensity on an accompanying timing task. Journal of Luman Movement Studies, 20, $123-131$.

Israel, L., Melac, M., Milinkeviteh, D., \& Dubos, G. (1994). Drug therapy and memory training programs: A double-blind randomized trial of general practice patients with age-associated memory impaiment. International Joumal of Psuchogeriatrics, 60), $155-170$.

Ivy, J.L., Costill, D.L., Bink, W.J., Lower, R.W. (1979). Influence of caffeine and carbohydrate feedings on endurance performatnee. Medicine and Science in sports, $11,6-11$

Jackson, J. L., Louwerens, J. W., Criossen, F, \& De Jong, H. T. P. (1992). Testing the effects of the imidazopyridine zolpidem on memory: an ccologically valid approach. Human Psychopharmacology _, 325330.

Jaffe, A. B., Thoran-Allerand, D., Greengard, P., \& Gandy, S. E. (1994). Estrogen regulates metabolism of Alzheimer amyloid B precursor protein. The Journal of Biological Chemistry. 269(18. Issue of May 6), $13065-13068$.

James, J.E. (1994). Does caffeine enhance or merely restore degraded psychomotor performance ? Biological Psychology/ Pharmacopsychology, 30, 124-125.

Janowski, D. S. \& Rausch, J. (1985). Biochenical hypothesis of premenstrual tension syndrone. Psychological Medicine. 1.5, 3-8.

Janowsky, D. S. \& Daris, J. M. (1970). Progestrone-estrogen effects on the uptake and release of norepinephrine by synaptosomes. Life Sciences. $2,525-531$.

Jarvis, M. (1993). Does caffeine intake enhance absolute levells of cognitive performance? Psychopharmacology, 110, 45-52.

Jellinger, K. Flament, H., Riederer, P., Schmnid, H., \& Ambrozzi, L. (1980). Levodopa in the treatment of (pre)senile dementia. Mechanisms of Aging and Development. 14, 41-45.

Jeukendrup, A., Saris, W. H. M., Brouns, F., \& Kester, A. D. M. (1996). A new validated endurance performance test. Medicine and Science in Sports and Exercise, 28, 266-270.

Johnson, L. C. (1982). Sleep deprivation and performance. In W. B. Webb (Ed.), Biological Rhythms, Sicep and Performance, . London: John Wiley \& Sons Ltd.

Jolles, J. (1986). Cognitive, emotional, and behavioral dysfunctions in aging and dementia. In D. F. Swaab, E. Fliers, M. Resmiran, W.A. wan Good, \& F. van Haaren, F. (Ed.), Progress in Brain Research, (Vol. 70, pp. 15-39). Amsterdam: Elseviers Science Publishers B.V.

Jolles, J., Howx, P. J., van Boxtel, M. P. J., Ponds, R. W. H. M. (1995a). The Maastricht Aging Study: determinanits of cognitive aging. Maastrich: Neuropsych Publishers.

Jolles, J., Verhey, F. R. J., Riedel, W. J, \& Howx, P. J. (1995b). Cognitive Impairment in Elderly People: Predisposing Foctors and Implications for Experimental Drug Studies. Drugs and.Aging. 76), 459-479.

Jones, G. M. M., Sahakian, B. J., Levy. R., Warburton, D. M., \& Gray, J. A. (1992). Effects of acute subcutaneous nicotine on attention, information processing and short-lerm memory in Alzheimer's disease. Psychopluarmacology. $108.485-494$.

Joseph, J. A. (1992). The putative role of liree radicals in the loss of neuronal functioning in senescence. Integr Physiol Bethay Sci. 27(3), 216-27.

Judd, H. L (1987). Oestrogen replacement therapy: physiological considerations and new applications. Baillienes Clinical Endocrinology and Metabolism. 1(1), 177-206.

Kampen, D. L., \& Sherwin, B. B. (1994). Estrogen use and verbal memory in healthy postmenopausal women. Obstetrics and Gynecology, 83(6), 979-83.

Kane, M.I., Hasher, L., Stoltzfus, B.R., Zacks, R.T., \& Connelly, S.L. (1994), Inhibitory attentional mechanisms and aging. Psuchology and Aging, 2(1), 103-112.

Kantor, H. I. Michael, C. M., \& Shore, H. (1973). Estrogen for older women. American Journal of Obstetrics and Gynecology, 4, 31-47.

Kauppila, A. (1996,). When should HRT be introduced? Associated problems. Paper presented at the 2nd International Symposium 'Women's Health and Menopause Risk Reduction Strategies', Florence (Italy), December 47 . 
Kausler, D. H. (1991). Experimental psychology. Cognition and Human aging. (2nd ed.). New York: SpingerVerlagg.

Kawas, C., Resnick, S., Morrison, A., Brookmeyer, R, Corrada, M., Zonderman, A., Bacal, C., DonnellLingle. D.. \& Mevter, E. (1997). A prospective studly of estrogen replacement therapy and the risk of developing Alzheimer's disease: the Baltimore longitudinal study of aging. Neurology $48($ June, $1517-1521$.

Kennealy, J. A., Mclennan, J. E., Loudon, R. G.; \& McLaurin, R. L. (1980). Hyperventilation-induced cerebral hypoxia. American Review of Respiratory Disease, 122, 407-412.

Kennedy, H. G. (1988) Fatigue and Fatigability. British Journal of Psychiatry. 153, 1-5.

Kida, M., \& Imai, A. (1993). Cognitive performance and event-related brain potentials under simulated thigh altitudes. Journal of Applied Psychology 74(4), 1735-1741.

Kimura ${ }^{\text {D., }}$ Hampson, E. (1994). Cognitive pattern in men and women is influenced by fluctuations in sex hormones. Current Directions in Psychological Science $3(2), 57-61$.

Kimura, D. (1995). Estrogen replacement therapy may protect against intellectual decline in postmenopausal women. Hormones and Behavior, $29(3), 312-21$.

King, D. J., \& Henry, G. (1992). The effect of neuroleptics on cognitive and psychomotor function. A preliminary study in healthy volunteers. British Joumal of Psychiatry. 160, 647.53.

Kirasic, K. C., Allen, G. L., Dobson, S. H., \& Binder, K. S. (1996), Aging, cognitive resources, and declarative learning. Psychology and Aging $11(4), 638-670$.

Kjellberg, A. (1977). Sleep deprivation and some aspects of performance. Waking and Sleep. 1, 139-143.

Klaiber, E. L., Kobayashi, Y., Broverman, D. M., \& Hall, 1. (1971). Plasma monoamine oxidase activity in regularly menstruating women and in amenorrheic women receiving cyclic treatment with estrogens and a progestin. Journal of Clinical Endocrinology and Metabolism. 33, 630-638.

Klaiber, E. L., Broverman, D. M., Vogel, W., \& Kobayshi, Y. (1979). Estrogen therapy for severe persistent depression in women. Archives of General Psychiatry. 36, 550-554.

Klaiber, E. L., Broverman, D.M. Vogel, W., Kennedy, J.A., Nadeau, C.J.L. (1982). Estrogens and the central nervous system: electroncephalography, cognition and depression. In R. C. Friedman (Ed), Behanior and the Menstrual Cycles (pp. 267-291). New York: Marcel Dekker inc.

Klatsky, A. L., Armstrong. M. A., \& Friedman, G. D. (1993). Coffee, Tea, and Mortality. Anmals of Epidemiology, 3(4), 375-381.

Kleijnen, J., \& Knipschild, P. (1992). Ginkgo biloba. The Lancet 340(8828), $1136-9$.

KIein, M., Ponds, R.W.H.M. Houx, P.J., \& Jolles, J. (submitted) Effect of test duration on age-related differences in Stroop Interference.

Klein, M., Ponds, R.W.H.M., Houx, P.J., \& Jolles, J. (submitted). The impact of aging on sustained attention.

Klein, M., Houx, P. J., \& Jolles, J. (1996). Long-tern persisting cognitive sequelae of traumatic brain injury and the effect of age. Joumal. of Nervous and Mental Disease. 184(8), 459-467.

Klein, M. (1997). Cognitive Aging. Altention and Mild Traumatic Brain Injury. Neuropsych Publishers, Neuropsych Publishers

Kok, A., Looren de Jong. H. \& Van Rooy, J. (1988). Elekwofysiologische kenmerken wan wisuele aandactit in jonge en oude profpersonen. In B. G. Deelman \& G. Mulder (Eds.), Bijdragen ann de expedimencele gerontologie on het onderzoek naar memtale belasting en stress (Vol. 3, pp 33-49). Amsterdami: Swets \& Zeitlinger.

Kopelman, M. D. (1986). The cholinergic neurotransmifter systern in human memory and dementia: A review. Special Issue: Human memory. Quarterly Journal of Experimental Psychology Human Experimental Psychology. 38(4), 535-73.

Koslowsky, M., \& Babkoff, H. (1992). Metaranalysis of the relationship between lotal sleep deprivation and performance. Chronobiology International. 2(2), 132-136.

Kovacs $\mathrm{E}$, Stegen J, Brouns $\mathrm{F}$ (in preparation) The effect of different dosages of caffeine on time trial cycling performance.

Kraaier, V., Van Huffelen, A., \& Wieneke, G. H. (1988). Changes in quantative EEG and blood flow velocity due to standardized hyperventilation; a model of transient ischaemia in young human subjects. Electroencephalography and Clinical Neurophysiology.70, 377-387.

Kraaier V. (1988). Human models for cerebral ischemia. Utrecht: Elink wijk BV.

Kraaier, V., wan Huffelen., A., \& Wieneke, G. H. (1989). The hyperventilation-induced ischaemia model in human neuropharmacology: meurophysiological and psychometric studies of aniracetam and 3-OH aniracetam. European Journal of Clinical Pharmacology $36(6), 605-11$.

Kraeplin, E. (1899). Psychiatrie. Leipzig: Barth, J.A. 
Kristensen, $V_{,}$Otsen, M. \& Theilgard, A (1977). Lewodopa treatment of presenile dementia. Acta Pswchiatrica Serandinaticica $55,41-45$.

Kugler, J.s Seus, R, Krauskopf, R., Brecht, H. M., \& Raschig, A. (1980). Differences in psychic performance with guanfacine and clonidine in normotensive subjects. British Journal of Clinical Pharmacology, 10 Suppl L. $71 \mathrm{~s}-80 \mathrm{~s}$.

Lader, M. (1985). Inirciduction to Psychopharmacology: A scope publication-Upjohn.

Lehman, J, Persson, S., Walinder, J., \& Wallin, L. (1981). Tryptophan malabsorption in dementia. Improvement in certain cases after tryptophan therapy as indicated by mental behavior and blood analysis. Acta Psychiatrica Seandinavion. 64. 123-131.

Levin, E. D., MoGurk, S. R., Rose, J. E. \& Butcher, L. L. (1990). Cholinergicmdopamimergic interactions in cognitive performance. Behav Neural Biol, 54(3), 271-99.

Levy, R. (1994). Age-associated cognitive decline. Working Party of the International Psychogeriatric Association in collaboration with the World Health Organization. Internation Journal of Psychogeriatrics. 6(1), 63-68.

Lezak, M. D. (1983). Neuropsychological Assessment (2nd ed.). New York: Oxford University Press.

Lezak, M.D. (1995). Neuropsychological Assessment (3rd edn.). New York: Oxford University Press

Landelijke Huisarts Vereniging (LHV), (1981). Yragenliist basiskeuring. [General Health Questionnaire] Amsterdan: Landelijke Huisar ts Vereniging.

Lichtman, R. (1996). Perimenopausal and postmenopausal hormone replacement therapy. Iournal of Nurses and Midwifery, 41(1), 3-28.

Lieberman, H. R., Wurtman, R. J., Emde, G. G., Roberts; C., \& et, a. I. (1987). The effects of low doses of caffeine on human performance and mood. Psychopharmacology, 92(3), 308-312.

Light, L. L. (1991). Memory and aging: four hypotheses in search of data. Annual Review of Psvchology. 43. 333-376.

Limouzin-Lamothe, M., Mairon, N., Joyce, C. R. B., \& Le Gal, M. (1994). Quality of life after the menopause: influence of hormonal replacement therapy. American Journal of Obstetrics and Gynecology $170,618-624$.

Linde, L. \& Bergstrom, M. (1992). The effect of one night without sleep on problem-solving and immediate recall. Psychological Research, 54(2), 127-36.

Lindenberger, U., \& Baltes, P'. (1994). Sensory functioning and intelligence in old age: a strong connection. Psychology and Aging. 9, 339-355.

Lines, C. R., Preston, G. C., Dawson, C. E., Brazell, C., \& Traub, M. (1991). The effects of pre-treatment with enalapril maleate on scopolamine-induced cognitive deficits in healthy volunteers. Journal of Psychopharmacologx. 5(3), 228-233.

Lingerfelser, T, Overkamp, D., Renn, W., Hamster, W., Boughey, J., Eggstein, M., \& Jacober, B. (1992). Cognitive and Psychomotor Function during severe insulin induced hypoglycemia in insulin-dependent patient. Neuropsychebiology, 25, 161-165.

Loke. W.H. (1988). Efffects of caffeine on mood and memory. Physiology and Behavior, 44, 367-372

Lopes, P., Mensier, A., Laurent, F. X. \& Besse, O. (1995). Traitement hormonal substitutif de la menopause et prevention des complicalions meurologiques. [Menopausal hormone replacement therapy and prevention of neurologic complications]. Contraceptives Fertilité ent. Sexualité, 23(5), 319-322.

Lorist, M. M., Snel, J., \& Kok, A. (1994a). Influence of caffeine on information processing stages in well rested and fatigued subjects. Psychopharmacology 113(3-4), 411-421.

Lorist, M. M., Snel, J., Kok, A., \& Mulder, G. (1994b). Influence of caffeine on selective attention in well rested and latigued subjects. Psychophysiology $31,525-534$.

Lorist, M.M., Smel, J., Mulder, $G_{.}$\& Kok, A (1995). Aging, caffeine, and information processing: An eventrelated potential analysis. Electroencephalography and Clinical Neurophysiology 96(5), 453-467.

Luine, $V$. N. (1975). Estradiol increases choline acetyltransferase activity in specific basal forebrain nuclei and projection area of female rats. Experimental Neurology. 89, 484-490.

I.uine, V. N., Khychevskaya, R. I. \& McEwen, B. S. (1975). Effects of gonadal steroids on activities of monoanine oxidase and choline acetyltransferase in rat brain. Brain Research, 86, 293-306.

Latine, V. N. \& McEwen, B. S. (1977). Effect of estradiol on turnover of type A monoamine oxidase in brain. Journal of Neurochemistry. 28, $1221-1227$.

Luine, V. L., Park, D., Joh, T., Reis, D., \& McEwen, B. (1980). Immunochernical demonstration of increased choline acetyltunsferase concentration in rat preoptic area after estradiol administration. Brain Research. 191, $273-277$. 
Luine, V. N., \& McEwen, B. S. (1983). Sex differences in cholinergic enzymes of diagonal band nuclei in the rat preoptic area. Neuroendocrinology, 36, 475-482.

Luteijn, F., \& van der Ploeg, F. A. E. (1983). Handleiding Groninger Intelligentie Test omanual Groningen Intelligence Test]. Lisse, the Netherlands: Swets 8 Zeitlinger.

MacLennan, A. H., MacLennan, A. \& Wilson, D. (1993). The prevallence of oestrogen replacement therapy in South Australia. Maturitas. 16(3), 175-83.

Magos, A. L., Brewster, E., Singh, R., O'Dowd, T., Brincat, M., Studd, J. W. W. (1986). The effects of norethisterone in menopausal women on oestrogen replacenent therapy: a model for the premenstruall syndrome. British Journal of Obstetrics and Gynaecology 93, 1290-1296.

Maier-Lenz, H., Ringwelski, L., Klein, G.; \& Richter, W. (1980). Zur Frage der Wirksamkeitsprüfung von zerebralAktiven Arzneimitteln. [On the effectivity testing of cerebro-active dinugs]. Arzneimittel Forschung. $30,830-832$.

Mann, J. J., Kapur, S., Schatzberg, A. F., Schwartz, J. C. \& Willner, P. (1995). A dopaminergic hypothesis of major depression. Clinical Neuropharmacology 1 (18/suppl), S57-565.

Mattilia ${ }_{\text {M. }}$. J., Nuotto, E. (1983). Caffeine and theophylline counteract diazepam effects in man. Mexhical Biology.61(6), 337-334.

Mattson, M. P, Barger, S. W., Cheng, B., Lieberburg, I, Smith-Swintosky, V. L., \& Ryder, R. E. (1993). Bamyloid precursor protein metabolites and loss of neuronal calcium homeositasis in Alzheimer's disease. Trends in Neuroscience. 16, 409-415.

Maurizi ${ }_{1}$ C. P. (1990). The therapentic potential for tryptophan and melatonin: possible roles in depression, sleep, Alzheimer's disease and abnomal aging. Med Hypotheses Marchi 31 (3), 233-242.

McCann, U. D., Penetar, D. M., Shaham, Y., Thorne, D. R., Gillin, J. C., Sing, H. C., Thomas, M. A.n \& Belenky, G. (1992). Sleep deprivation and impaired cognition. Possible role of brain catecholamines. Biological Psychiatry. 31(11), 1082-97.

McDowd, J. M., \& Birren, J. E. (1990). Aging and attentional processes. In J. E. Birren \& K. W. Schaie (Eds.), Handbook of the Psychology of Aging, (3rd ed., pp. 222-233). New York. Van Nostrand Reinhold Company.

McEntee, W. J. \& Crook, T. H. (1990). Age associated memory impairment: a role for catecholamines. Neurology, 40, 526-530.

McEntee, W. J., \& Crook, T. H. (1991). Serotonin, menory, and the aging brain. Psychopharmacology Berlin, $103(2), 1.43-9$.

McEwen, B. S., Alves, S. E. Bulloch, K., \& Weiland, N. G. (1997). Owarian steroids and the brain: implications for cognition and aging. Neurology, 48(Suppl 7), 58-514.

McFarland, R.A. (1963). Experimental evidence of mild hypoxia on mental performance assessed by a test of selective attention. Ergonomics, 6, 338-366.

McMorris, T., \& Keen, P. (1994). Effect of exercise on simple reaction times of recreational athletes. Perceptual and Motor Skills, $78(1), 123-30$.

Medisch en Maatschappelijk InformatieCentrum (Medical and Societall Information Centre)-MEMIC (1996). VMO-normwraagstellingen 1991. (Social Class Schedule).

Merryman, W. Boidman, R., Bames, K., \& Rothchild, 1. (1954). Progestrone "anaesthesia' in human subjects. lournal of Clinical Endocrinology and Metabolism. 14, 1567-1569.

Mewaldt, S. P., \& Ghoneim, M. M. (1979). The effects and interactions of scopolamine, physostigmine and methamphetamine on human memory. Pharmacology Binchemistry, and Behavior. 10(2), 205- 10.

Mikulincer, M., Babkoff, H., \& Caspy, T. (1989). The effects of 72 hours of sleep loss on psychological yariables. British Journal of Psychology, 80, 145-162.

Minors, D. S. Waterhouse, J. M. (1981). Circadian Rhythms and the Human Bristol: Wright PSG.

Mishima, K., Okawa, M., Hishikawa, Y., Hozumi, S., Hori, H. \& Takahashi, K. (1994). Morning brigh light therapy for sleep and behavior disorders in elderly patients with dementia. Acta Psychintrica Scandinavica, Jan: $89(1), 1-7$.

Mohr, E., Schlegel, J., Fabbrini, G, \& al, e. (1989). Clonidine treatment of Alzheimer's disease. Archives of Neurology. 46, 376-378.

Mohr, E., Knott, V., Herting, R. L., \& Mendis, T. (1993). Cycloserine treatment of Alzheimer's disease. Neuropsychopharmacology. 9(Supplement). $96 \mathrm{~S}-97 \mathrm{~S}$.

Moja ${ }_{2}$ E. A., Lucini, V., Benedetti, F., \& Lucca, A. (1996). Decrease in plasma phenylalanine and tyrosine afler phenylalanine-tyrosine free amino acid sollutions in man. Liffe Sciences. $58(26), 2389-95$. 
Molina, J. A, Bermejo, F, del Ser, T., Jiménez-Jiménez, F. J., Herranz, A. Fernandez-Calle, P., Ortuno, B., Villanue $\mathrm{w}_{3} \mathrm{C}$, \&ainz, M. J. (1994). Alcoholic cognitive deterioration and nutritional defreiencies. Acta Meurological scandinavicas $89,3,84-390$.

Moran, A. (1996) The Psychology of Concentration in Sports Performers. The Psychologist.

Morris, A. M., So, Y., Lee, K. A. Lash, A. A. \& Becker, C. E (1992). The P300 event-related potential. The effects of sleep deprivation. Ioumal of Oceupational Medicine. $34(12), 1143-52$.

Mortel; K. F. Meyer, J. S. (1994) Protecteur des oestrogenes. Alyheimer Actualités, 84, 5-6.

Mortimer, J. A. (1994). What are the risk factors for dementia ? In F. A. Huppert, C. Brayne, \& D. W. O'Connor (Eds.), Dementia dud Normal Aging. (pp. 208-229). Cambridge: Cambridge University Press.

Murphy, D. L., Henry, G.M. (1972). Catecholamines and memory: enhanced verball learning during LDOPA administration. Psychopharmacologr, 27(4), 319-26.

Murin, K. R. \& Nagarajan, T. M. (1974). Hyperventilation and psychometric testing. A preliminary study. Anassithesia, 29, 50.58 .

Myers, B. L., \& Badia, P. (1995). Changes in circadian thythns and sleep quality with aging: mechanisms and interventions. Weurossience and Biobehavioral Reviews. Winter 12(4), 553-571.

Nehlig. A., Daval, J., Be Debry, G. (1992). Caffeine and the central nervous system: mechanisms of action, biachemical, metabolic, and psychostumulant effects. Brain Research Reviews, 17, 139-170.

Nehlig, A., \& Debry, G. (1994). Caffeine and sports activity: a rewiew. International Journal of Sports Medicine, 15, 2115-223.

Newliouse, P. A., Sunderland, T., Tariot, P. N., Blumhardt, C. L., Weingartner, H., Mellow, A., \& Murphy. D. L. (1988), Intravenous nicotine in Alzheimer's disease: a pilot study. Psychopharmacology . 25, 171-175.

Newhouse, P. A., Penetar, D. M., Fertig, J. B., Thorne, D. R., \& et al. (1992). Stimulant drug effects on performance and belavior after prolonged sleep deprivation: $A$ comparison of amphetarnine, nicotine, and deprenyl. Special Issue: Stimulants to ameliorate sleep loss during sustained operations. Milliary Pisychology. 4t(4), 207.233.

Niatoh, P. (1976). Sleep deprivation in human subjects: a reappraisal. Waking and Sleeping, 1, 53-60.

Nideffer, R. (1979) Predicting human behavior: a theory and a test of atlentional and interpersonal stvle. San Diego, CA: Enhanced Performance Associates.

Niklokov, N, Patchev, V., Kisselkova, E., \& Harleva, S. (1991). Late afterweffects of hyperventilation or breathing exercises on cognitive functions. Homeostasis, 33(4), 207-208.

Nilsson, L. G., Bäckman, L., \& Karlisson, T. (1989). Priming and cued recall in elderly, alcohol intoxicated and sleep deprived subjects: a case of functionally similar memory deficits. Psychological Medicine 19(2), 42333.

Nilsson, L. G., Bäckman, L., Erngrund, K., Nyberg, L., Adolfsson, R., Bucht, G., Karlsson, S., Widing, M., \& Winblad, B. (1997). The Betula prospective cohort study: memory, health, and aging. Aging, Leuropsychology and Cognition 4. (1), 1-32.

Noble, J., Jones, J. G., \& Davis, E. J. (1993). Cognitive function during moderate hypoxemia. Anesthesiology and Intensive Caile, $21(2), 180-4$.

Nordberg, A., Lilja, A.., Lundqvisi, H., Hartwig, P., Amberla, K., Viitanen, M., Warpman, U., Johansson, M., Hellstrom, L. E., Bjurling, P. \& et, a. I. (1992). Tacrine restores cholinergic nicotinic receptors and glucose mitubolism in Azheimer patients as visualized by positron emission tomography. Neurobiology of Aging. $13(6), 747.58$.

Nordberg, A. (1996). Functional studies of new drugs for the treatment of Alzheimer's disease. Acta Neurologica Sciundimarical 16 (Supplement), 137-144.

Nordberg, A., Hertwig, P., Lilja, A, \& et 1 . (1990). Decreased uptake and bindling of 11C-nicotine in brain of Alzheimer's patients and visualized by positton emission tomography. Joumal of Neural Transmission.2. 21.5-224.

Norman, D.A. (1979), Memory and Attention An introduction to human information processing. New York: Willey.

O'Hanlon, J. F. \& Horvath, S. M. (1973). Neuroendocrine, cardiorespiratory, and performance reactions of hypoxic men during a monitoring task. Aerospace. Medicine, 44(2), 129-34.

Olikura, T., Isse, K. Akazawa, K., Hamamoto, M., Yaoi, Y., \& Hagino, N. (1994b). Low-dose estrogen teplacement therapy for Alzheimer disease in women. The Journal of the North American Menopause Socien 1 (3). 125-130.

Ohkura, T., Isse. K., Akazawa, K., Hamamoto, M., Yaoi, Y., \& Hagino, N. (1994a). Evaluation of estrogen treatment in female patients with dementia of the Alzheimer type. Endocrine Journal, 41 (4), 361-371. 
Ohkura, T., Isse, K. Akazawa, K., Hamamoto, M., Yaoi, Y., Hagind, N, (1995), Long-ferm estrogen

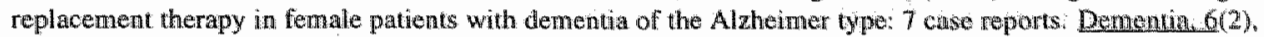
99.107.

Ollat. H. (1992). Dopaminergic insufficiency reflectimg cercbral aging: value of dopaminergic agonist piribedil. Journal of Neurology, $239,513-6$.

Oosterink, B.J., Stienstra, C.M., Nyakas, C., Ouma, B. R. K., Korf, I., \& Luiten, P. G. M. (1997, June 24, 1997). Neuropnotextive effects of 5-HT1a agonists agains NMDA-induced cell doath of cholinegge nourons and the putative role of corticosterone. Paper presented at the NWO: First Dutoh endo-netro meeting, Hote: de Branding, Doorwerth, the Netherlands.

Oswald, I. (1980). Sleep as a restorative process; human clues. Progress in Brain Research, 53, 279288.

Ont, A., Breteler, E. B., Birkenhauser-Gilesse, E. B., var Harskamp, F, de Koning, I., Hofman, A. (1996), De prevalentie bij ouderen van de ziekte van Alzheimer, wasculare dementie en dementie bij de ziekte van Parkinson; het ERGO-onderzok. Nederlands Tjidschritt won Geneeskunde 140(4), 200-204,

Ottowitz, W. E \& Halbreich, U. (1995). Mood and Cognitive Changes Following Estrogen Replacement Therapy in Postmenopausal Women. CNS Drugs. 4(3), 161-167.

Overall, J.E. \& Rhoades, H.M. (1987). Adjusting p values for multiple tests of significance. In: Meltzer HY (ed) Psychopharmacology. The third Generation of Progress. Raven Press, New York, pp 1013-1018

Paas, F. G., \& Adam, J. J. (1991). Human information processing during physical exercise. Butgonomics $34(11), 1385-97$

Paganini-Hill, A. Henderson, V. W. (1994), Estrogen deficiency and risk of Alzheimer's disease in women. Americian Journal of Epiderijology, 140(3), 256-261.

Palmer, A. M., \& DeKosky, S. T. (1993), Mononmine neurons in aging and Alzheimer's disease. LNeurall Trainsm Gen Sect. $91(2-3), 135-59$

Park, D. C., Smith, A. D., Lautenschlager, G., Earles, J. L., Frieske, D., Zwahr, Mw \& Gaines C. L. (1996). Mediators of long-term memory performance across the life span. Psychalogy and Aging, 11(4),621.637.

Park, S. B., Coull, J. T., McShane, R. H., Young, A. H., Sahakian, B. J., Robbins, T. W., \& Cowen, P. IJ. (1994). Tryptophan Depletion in Normal Volunteers Produces Selective Impaiments in Learning and Memory. Neuropharmacology 33(3/4), 575-588.

Paterson, M. E. (1982). A randomized, dowble-blind, cross-over study into the effect of sequential mestramol and norethisterone on climacteric symptoms and biochemical parameters. Maturitas, 4(2), 83-94.

Pathy, M. S. J. (1993). The pharmacologieal management of cognitive impairment iru the demented patient. Progress in Neuro-Psychopharmacology and Biological Psychiatry, 17(1), 515-524.

Pepeu, G. (1994). Memory disorders. novel treatments, clinical perspective. Life Sciences. $552(25-26), 2189-94$.

Perlmuter, M. \& Nyquist, L. (1990). Relationships between self-reported physical and mental health and intelligence performance across adulthood. Journal of Gerontology: Psychological Sciences, 45, P145-P155.

Perty, A. R., \& Wingfield, A. (1994), Contextual encoding by young and elderly adults as revealed by cued and free recall. Aging and Cogmition, $1(2), 120-139$

Peruche, B., \& Kreglstein, J. (1993), Mechanisms of arug actions against neuronal damage caused by ischemia - an overview. Progress in Neuro-Psychophamacology and Biological Psychiatry, 12(1), $21-70$.

Petitt. N. Etgen, A. M. (1990). Alphal-adremoneceptor agmentation of beta 4 stimulated cAMP formation is cuhanced by estrogen and reduced by progestrone in ret hypothatamic slices. Loumal of Neuroseduce $10(8)$. $2842-2849$.

Pfaff, D. W. (1980). Estroger and brain function. New Youk: Springer Verlag.

Phillips, S. M. \& Sherwin., B. B. (1992). Effects of estrogen on memory function in surgically methoptusal womer. Psychoneuroendocrinology $17(5)$. 485-495.

Picton, T. W., Stuss, D. T., Champagne, S. C. \& Nelson, R. F. (1984). The effects of age on human ewentrelated potentials. Psychophysiology $21(3), 312-325$.

Pollack, 1., \& Norman. D. A. (1964). A non-parametric analysis if recognition experiments. Psychonomic Siciences, 1, 125-126

Polster, M. R., MoCarthy, R. A., O'Sullivan, G., Gray, P. A., \& Park, G. R. (1993). Miderolam-induced amnesia: inplications for the implicitexplicit memory distinction. Brain and Cognition, 22(2), 24465.

Ponds, R.W.H.M., Browwer, W.H., \& Van Wolffelaar. P.C. (1988). Differences in divided attention in a simulated driwing task. Lournal of Gerontology, 43, $151-156$.

Pons, L., Trenque, "T. Bielecki, M., Moulin, M. \& Potier, I-C. (1987). Attentional offecis of caffeine in man: comparisons with drugs acting upon performance. Psychiatry Research. 23, 329-333. 
Poon, L. W. (1993). Assessing neuropsychological changes in pharmacological trials. Clinical Neuropharmadogy $16(1), \$ 31-8$.

Powers, 5. K. Martin, D., \& Dodd, S. (1993). Exercise-induced hypoxemia in elite endurance athetes. lincidence, wauses and impact on Vo2mas. Spors Medicine 16(1), 14-22.

Prinz; P. N., Vitiello, M. W. (1993). Sleep Disorders and Insomnia in the elderly. Facts and Research in Geronlologer 2. $55-65$.

Pujol, M. Rebillard, Pued, Lenoir, Eybalin, \& Recasens: 1990) Glutamate neurotoxicity in the cochlea: a posstble conseguence of ischemice or anoxic conditions oceurring in ageing. Acta Otolaryngology Supplement Stockhollm. 476,326 .

Ruffacle, K. C., Berandi, A. Pearse-Morris, P., Asthana, S. Haxby, J. V., Shapiro, M. B., Rapoport, S. I. \& Soncrant, T. T. (1991). Effects of acute infusion of the muscarinic cholinergic agonist arecoline on werbal. and visuo-spatial function in dementia of the Alzheimer type. Progress in Neuro-Psychopharnacology and Biological Psychatary 115, 643,648.

Ralun, H., Ots, A. B., Htodge, M., Epstein, M. A., Hunter, S. W., \& Fenn, W. O. (1946). The effects of hypocspnia on performance. Axiation and Medicine. 16, 164-172.

Rainbow, T. C., Davis, P. G. \& McEwen, B. S. (1980). Brain Research, 194(2), 548-555.

Rammsayer, T. (1989). Is there a common dopaminergic basis of time perception and reaction time? Neuropsechobiology. $21(1), 37.42$.

Rammsayer, T. (1992). Effects of benzadiazepinewinduced sedation on temporal processing. Human Psychopioarmacodogy. $7,311.318$.

Rauramo, L., Lagerspete, K., Engblom, P. \& Punnonen, R. (1975). The effect of castration and peroral estrogen therapy on some psychological functions. Frontieres of Hormone Research, 3, 94-104.

Rediess, S., \& Caine, E. D. (1996). Aging, Cognition, and DSM IV. Aging Neuropsuchology, and Cognition. Luin, $3(2), 105-117$.

Resnick, S. M., Trotmant, K. M., Kawas, C., \& Zonderman, A. B. (1995). Age-associated changes in specific errors on the Benton Visual Retention Test. I Gerontal B Psychol Sci Soc Sci. 50(3), P171-8.

Rey, A. (1964). L'examen nsychologique dans les cas dencéphalopathie iraumatique (Psychological assessment in cases of traumatic brain injury). Paris: Presses Universitaires de France.

Riedel, W. J. \& Jolles, J. (1996). Cognition Enhancers in Age-Related Cognitive Decline. Drugs and Aging B(4), 245.274 .

Riedel, M., Rafflenbeul, W., \& Lichten, F. (1993). Owarian sex steroids and atheroselerosis. The Clinical Investigator $71,406-412$.

Riedel, W. J., Hogervorst, E., Leboux, R. L. A. M., Verhey, F. R. J, Praag, H. M. v., \& Jolles, J. (1995a), Caffeine enhances Memory after Cholinergic Blockade. Psychophamacology, 122(2), 158-168.

Riedel, W. J., Peters, M. L., Van Boxtel, M. P. J., \& OHanlon, J. F. (1995c). The Influence of Piracetam on Actuat Driving Behavior and Postural Stability of Elderly Subjects. In W. J. Riedel (Ed.) Cognition Eabancing Drugs. Cholinergic Function and Agewelated Decline (pp. 95-108). Maastricht: Neuropsych Puiblishers.

Riedel. W. J., Hoggervorst, E., Verhoy, F. R. J. \& Jolles, J. (1997). Measuring Cognitive Abilities in Cholinergic Dysfunction. In I. Hindmarch \& P. D. Stonier (Eds.), Human Pychopharmacology: Mesasures aud Methods (Vol. VI, pp. 29-56). Chichester: Wiley.

Riedel, W. J., Hogervorst, E., \& Jolles, J. (1995b). Cognition Enhancers and Aging. In J. Jolles, P. J. Houx, M. P. J. Van Boxtel, \&. R. W. H. M. Ponds (Eds.), Maastricht Aring Study: Determinants of Cognitive Aging (pp. 149-156). Maustricht, The Netherlands: Neuropsych Publishers.

Riedel, W., Klaassen, T., Deutz, N., Honig, A., Van Someren, A., \& Van Praag, H. (submitted). Tryptophan Depletion in Normal Volunteers Produces Selective Impairnent in Memory Consolidation. Iourmal of Psychopharmacology.

Roache, J. D. \& Griftiths, R. R. (1987), Interactions of diazepam and caffeine: Behavioral and subjectiwe dose effects in humans. Pharmacology Biochemistry and Behavior. 26(4), 801-812.

Robbins T. Everitt B (1995) Arousal Systems and Attention. In: Gazzaniga M (ed) The Cognitive Neurosciences. MTT Press, Cambridge MA (USA), pp 703-720

Robinson, D., Friedman, L. Marcus, R., Tinklenberg, J., \& Yesavage, J. (1994). Estrogen replacement therapy and memory in older womem. Journal of the American Geriatrics Society 42(9), 919-922.

Rogers, P.J., Richardson, N.J., \& Dernoncourt, C. (1995). Caffiene use: is there a net benefit for psychomotor performance. Neuropsychohiology, 31, 195-199. 
Rother, M., Kessler, J., Funke, M., Rother, I., \& Windisch, M. (1994). Memory imparment after hyperwentilation-a physiological model of cogmitive dysfunctions. Neurobiology of Aging 15 (1), 92 .

Rowe, J. W. \& Kahn, R.L. (1987). Human aging: Usual and successful Science. 237. 1434149

Rowley, B., Van, F. Mortimore, C, \& Connell, J. (1997). Effects of Acute Tryptophan Deplecion on Tests of Frontal and Temporal Lobe Function: Journal of Psychopharnacology. $11(3)$, A60.

Rushmer, F. R., Boothby, W. M., \& Hinshaw, H. C. (1941). Some effects of hyperventilation with special reference to aviation medicine. Proceedings of the stafif meetings of the Mava Clinic. 16. $801-808$.

Sackett, D. L., Haynes, R. B., Gyuat, G. H., \& Tugwell, P. (1991). Clinical epidemiology: a basicic science for clinical medicine. (2nd ed.). Boston: Little, Brown and Company.

Sahakian, B. J., Jones, G., Levy, R., Gray, J., \& Warburton, D. (1989). The effects of nicotine on attention, information processing and short-term memory in patients with dementia of the Alzheimer's type. Britisti Journal of Psychiatry. 154, 797-800.

Sahakian, B. J., Owen, A. M., Morant, N. J., Eagger, S. A., Boddington, S., Craytton, L., Crockford, H. A., Crooks, M., Hill, K., \& Levy, R. (1993). Further analysis of the cognitive effects of tetrahydroaminoacridine (THA) in Alzheimer's disease: assessment of attentional and mnemonic function using CANTAB. Psychopharmacology Berl, 110(4), 395-401.

Salamone, L. M., Pressman, A. R., Seeley, D. G., \& Cauley, J. A. (1996). Estrogen replacement therapy. A survey of older women's attitudes. Archives of International Medicine. 156(12), 1293-7.

Saletu, B., Grunberger, J., Anderer, P., \& Linzmayer, L. (1996a). Effects of the nowel neuroprotective agent" riluzole, on human brain function and behavior: 1. Double-blind, placebo-controlled EEG mapping and psychometric studies under normoxia. Methods and Findings in Expermental and Clinical Phamacology $18(1), 55-66$.

Saletu, B., Grunberger, J., Anderer, P., \& Linzmayer, L. (1996b). Effects of the novel neuropratective ageut. riluzole, on thuman brain function and behavior: $\mathbb{I 1}$. Double-blind, placebo-controlled EEG mapping and psychometric studies under hypoxia. Methods and Findings in Experimental and Clinical Pharmacology. $18(1), 67-81$.

Salletu, B., Grumberger, J., Anderer, P., Linzmayer, L.n \& Konig, P. (1996c). On the cerebro-protective effects of caroverine, a calcium channel blocker and antiglutaminergic drug: double-blind, placebo-controlled, EEG mapping and psychometric studies under hypoxia. British Journal of Chinical Pharmacology 41(2), 89-99.

Saletu, B., Schulz, H., Herrmann, W. M., Anderer, P., Shrotriya, R. C., \& Vanbrabant, E. (1994). BMS181168 for protection of the human brain against hypoxia: Double-blind, placebo-controlled EEG mapping studies. Pharmacopsychiatry 27(5), 189-197.

Saletu, B., Grunberger, J., \& Anderer, R. (1990). On brain protection of co-dergocrine mesylate (Hydergine) against hypoxic hypoxidosis of different severity: double-blind placebo-controlled quantitative EEG and psychometric studies. Int I Clin Pharmacol Ther Toxicol.28(12), 510-24.

Saletu, B, \& Grünberger, J. (1984). The hypoxia model in human psychopharnacology: neuropsychological and psychometric studies with aniracetam i, v.. Human Neurobiology. 3, 171-181.

Salmasso, D. (1993). Memory and aging: components and processes. Functional Neurology. $8(3), 165-183$.

Salmela, J. H., \& Ndoye, O. D. (1986), Cognitive distortions during progressive exercise. Percieptual and Motor Skills, 63(3), 1067\%1072.

Salthouse, T. A. \& Babcock, R. L. (1991), Decomposing adult age differences ila working memory. Developmental Psychology, 27, 763-776.

Salthouse, T. A., \& Coon, Y. E. (1993). Influence of task-specific processing speed on age differences in memory. Journal of Gerontology: Psychological Sciences, 48(5), P245-P255.

Salthouse, T. A. (1985). Speed of behavior and its implications for cognition. In J. E. Birren \& K. W. Schaie (Eds.), Haandbook of the psychology of aging. (pp. 400-426). New York: Van Nostrand Reinhold.

Salthouse, T. A. (1994). Aging assaciations: influence of speed on adult age differences in assuciative learning.

Learning. Memory. and Cognition. 20. $1486-1503$.
Salthouse, T.A. (1988). Resource reduction interpretations, of cognitive aging. Brain and Cogmilion. $2,238-272$.

Salthouse, T. A., \& Lichty, W. (1985). Tests of the neural noise hypothesis of age-related cognitive change. Journal of Gerontology. 40(4), 443-450.

Salthouse, T.A. Davenport Rogan. J., \& Prill, K.A. (1984) Division of Altention: Age-differences on a visually presented memory task. Memory and Cognition. 12(6), 613-620.

Salthouse, T. A. (1985). A Theory of Cognitive Aging. Amsterdam: Norih-Hollamd.

Salthouse, T. A., Kausler, D. H., \& Saults, J. S. (1990). Age, self-assessed health status, and cognition. Joumall of Gerontology: Psychological Sciences. 45 (P156-P160). 
Sanders, A. . (1983). Towardls a model of etress and hünan perfornance. Acta Psychologica 53 (1), 61-97.

Sarte, P. M. (1989). Effects of ovarian steriods on the cardiovascular system. In J. Ginsberg (Ed.). The Circulation of the Female from the fitatdle to the Grave (pp. 112-140): Carnford Parthenon Publishing.

Sarrel. P. M. (1990). Ovarian hormones and the circulation. Maturitas, 590, $287-298$.

Sarter, M. Schtieider, H. H. Stephens, D. N. (1988) Treatment strategies for senile dementia: Amtagonist bcarbolines. Trends in Newrosciences. $111(1), 13-17$.

Satz, P. (1993). Brain reserve capacity on symptom onset after brain injury: A formulation and rewiew of ewidence lifor threshold theory. Neuropswchollogy 7(3), 273-295.

Schachter, S., Singer, I. E. (1962). Cognitive, soevial, and physiological determinants of the emotional state. Pexchological Review $69,379.399$.

Schalfer, K. Gorman, E, \& Arnold, H. (1981). A pilat study to evaluate the effect of acute and long-term admimistration of cyclandelate on the wigilance of subjects submitted to hypoxic studies. Arzneinittel Forshung/Drug Research, 31 $(1,6)$. $1032-1035$.

Schaffler, K., K Klausnitzer, W. (1988). Randomized placebo-controlled double-blind cross-over study on antihypoxidotic effects of piracetam using psychophysiological measures in thealthy wolunteers. Arzneimitrel Forsclyune $38(2), 288-91$.

Schaffler, K. Wauschkulim, C. H., \& Hauser, B. (1991). Study to evaluate the encephalotropic potency of a hemodialysate. Controlled study using electro-retinography and visual evoked potentials under hypoxic conditions in human wolunteers (preliminary conmumication). Arzneimittel Forschung 41(7), 699-704.

Sichaffler, K. Reeh, P.W. (1985). Doppelblindstudie zur hypoxieprotektiven Wirkung eines standarisienten Ginkgo-Biloba-Präparates nack Mehrfachwerabreichung an gesunden Probanden. Arzmeimittel Forschung Drug Research, 35(8), 1283-1286.

Schlaepfer, T. E. Bartsch, P., \& Fisch, H. U. (1992). Paradoxical effects of mild hypoxia and moderate altitude on human visual perception. Clinical Science 83(5), 633-6.

Schmidt, U., Brendemuhl, D., Engels, K., Sehenk, N., \& Ludemann, E. (1991). Piracetam in elderly motorists. Pharmacopsychiatry $24(4), 121-126$.

Schmidt, $\mathbb{R}$., Fazekas, F., Reinhart, B., Kapeller, P.. Fazekas, G., Offenbacher, H., Eber, B., Schmacher, M., \& Freidl, W. (1996). Estrogen replacement therapy in older women: a neuropsychological and brain MRI study. Jourmal of the American Geriatrics Society. 44(1.1), 1307\%1313.

Schmitt, J. A. J., Jorissen, B., Sobczak, S., Van Boxtel, M. P. J., Deutz, N. E. P., \& Riedel, W. J. submitted). Tryptophan depletion impairs nemory consolidation, but improwes focused attention. Nature.

Schneck, M. K. (1983). Nootropics. In B. Reisberg (Ed.), Alzheimer's Disease, (pp. 362-368). New York: The Free Press.

Schneider, M. A., Brotherton, P. L., \& Hailes, J. (1977). The effect of exogenous estrogens on depression in menopausal women. Medical Joumal of Australia 2(5), 162-163.

Schneider, L. S., Farlow "M. R., Henderson, V. W., \& Pogoda, I. M. (1996). Effects of estrogen replacement therapy on response to tacrine in patients with Alzheimer's disease. Neurology, 46(6), 1580-1584.

Schwart2, B. L., Hashtroudi, S, Herting, R. L., Seluwartz, P., Deutsch, S. I. (1996), d-Cycloserine enhances implicit menory in Alzheimer patients. Newology, 46(2), 420-4.

Selkoe, T. (1993). Aging brain, aging mind. Mind and Brain: Readings from Scientific American Magazine (pp. 101-112) W.H. Freeman \& Co Publishers: New York.

Senin, U., Albate, G., Fieschi, C., Gori, G., Guala, A., Marini, G., Villardita, C., \& Parnetti, L. (1991). Aniracetgm (Ro $13-5057)$ in the treatment of senile dementia of the Alzheimer's type: results of a placebo controlled multicentre clinical study. European Journal of Neuropsychopharmacology 1, 511-517.

Sharma, A. C.. \&ulkami, S. K. (1992). Evaluation of learming and memory niechanisms employing elevated plus-mare in rats and mice. Progress in Neuropsychopharmacology and Biological Psychiatry, 16(1), 117-25.

Shaver, J. L. (1994). Beyond hormonal therapies in menopausu. Experimental Goromtology 29 (3-4), 469-76.

Sherwin, B. B. (1988). Estrogen and/or androgen replacement therapy and cognitive functioning in surgically menopausat women. Psychoteuroendocinologw. 13(4), 345-57.

Sherwin, B. B. (1994), Sex hormones and psychological functioning in postmenopausal women. Experimental Geroutologer. 22(3-4), 423-30.

Sherwin, B. B. (1994). Estrogenic effects on memory in women. Annals of the New York Academy of Sciences. 743.213-30.

Sherwin, B.B. (1996). Hormones, mood, and cognitive functioning in postmenopausal women. Obsterics and Gynecology. 82,20 S-265. 
Sherwin, B.B. (1991). The impact of different doses of estrogen and progestin on mood and sexual bethavior in postmenopausal women. Journal of Clinical Endocrinology and Matabolism, 220), 336-343.

Sherwin, B. B. \& Phillips, S. M. (1990). Estrogen and Cognitive Functioning in Surgically Menopausal Women. Amnals of the New York Academy of Sciences, $592,474-475$.

Sherwin, B. B. \& Suranyi-Cadotte, B. B. (1990). Up-regulatory effect of estrogen on platelet 34 imipramine binding sites in surgically menopausal women. Biological Psychiatry. 28, 339-348.

Sherwin, B. B. (1997). Estrogen effects on cognition in menopausal women. Newrology. May 48 45 Suppl 7), S2Im S26.

Siegfried, K. R. (1993). Pharmacodynamic and early elinical studies with velnacrias: Acta Neurologica Scandinavica Suppl. 149. 26-8.

Silverman, K. Evans, S.M., Strain, E.C., \& Griffiths, R.R. (1992). Withdrawal syndrome after the double blind cessation of caffeine consumption. The New England Journal of Medicine 327 (16), 1109-1113.

Simpkins, J. W. Singl, M., \& Bishop, J. (1994). The potential role for estrogen replacement therapy in the treatment of the cognitive decline and newrodegeneration associated with Alzheimer's disease. Fisher Symposium: Future directions in Alzheimer's disease research (1994, Washington, DC). Neurobiologyof Aging, 15(Suppl 2), S195-S197.

Smith, A.P., Kendrick. A., Maben, A. (1992). Use and effects of food and drinks in relation to dialy thythms of mood and cognitive performance. Proc Nutr Soc Dec.51 (3), 325-333.

Smith, B.D., Rafferty, J., Lindgren, K., Smith, D.A., \& Nesper, A. (1991). Effects of habitual calfeine use and acute ingestion: Testing a biobehavioral model. Physiology and Behavior, 51, 131-137.

Smith, D. F., Stomgren, E., Petersen, H. N., Willians, D. G., \& Sheldon W. (1984). Lack of effect of tryptophan treatment in demented gerontopsychiatric patients. Acta Psychiatrica Scandinavica $70,470,477$.

Smith, J., \& Battes, P. B. (1993). Differential psychological ageing: profilles of the old and the wery old. Ageing and Society. $13,551-587$.

Smith, A. (1968). The Symbol digit Modalities Test: a neuropsychological test for economic screening of learning and other cerebral disorders Learning Disorders. 36, 83-91.

Smith, A., \& Maben, A. (1993). Effects of sleep deprivation, lunch and personallity on pertormance, mood and cardiovascular function. Physiology and Behavior. 54, 967-972.

Smith A, Kendrick A, Maben A, Salmon J (1994) Effects of breakfast and caffeine on cognitive performance, mood and cardiovascular functioning. Appetite. 22, 39-55

Smith, C. (1984). Aging and changes in cerebral energy metabolism. Trends in Neuroscience (June).

Soetens, E., Casaer, S., D'Honge, R., \& Hueting, J. E. (1995). Effect of amphetamine on long-term retention of verbal material. Psychopharmacology, $112(2), 155-62$.

Soetens, E., D'Hooge, R., \& Hueting, J. E. (1993). Amphetamine enhances human-memory consolidation. Neuroscience Letters, 161(1), 9-12.

Sommer, B. (1982). Cognitive behavior and the menstrual cycle. In R. C. Friedman (Ed.), Behaviorand the mensitruall cycle, (pp. 105-123). New York: Marcel Dekker, Inc.

Sourander, $\mathbb{L}, \mathbb{B}$., Portin, $\mathbb{R}$, Molsa, P., \& al, e. (1987). Senile dementia of the Alzheimer's type treated with aniracetam. Psychopharmacology, 91, 90-95.

Spiegel, R. (1995). Psychopharmacology: an introduction. (3rd ed.). Chichester: John Willey and Sons

Spiegel, R. (1989) Psychopharmacollogy: an introduction (2nd ed.) Chichester: John Wiley \& Sons LTD.

Squire, L. R. (1987). Memory and Brain. Newyork: Oxford University Press.

Stampfer, M. J, \& Colditz, G. A. (1991). Estrogen replacenemt therapy and coronary hearl disease: a quantitative assessment of the epidemiologic evidence. Preventive Medicine 20(1), 47-6.3.

Stern "Y. A., Alexander, G., Prohovnik, I., \& Mayeux, R. (1991). Education provides a cognitive reserve against the clinical manifestations of Alzheimer's disease: evidence from regional cerebral blood flow. Society for Neurosicience Abstracts 17. 1258.

Sternberg, S. (1969). Memory scanning: mental processes revealed by reaction time experinents. American Scientist. 57(4), 421-457.

Steyvers, F. J., \& Gaillard. A. W. (1993). The effects of sleep deprivation and incentives on human performance. Psychological Research. 55(1), 64-70.

Stoddart, J. C. (1967). Electroencephalographic activity during voluntarily controlled alveotar hyperventitation. British Journal of Anaestliesia, 39, 2-10.

Streufert, S., Pogash, R., Miller, J., Gingrich, D., Landis, R., Lonardi, L., Severs, W., \& Roache, J.D. (1995). Effects of caffeine deprivation on complex human functioning. Psychopharmacology. $118,377-384$.

Sunderland, T., Tariot, P. N., Cohen, R. M., Weingartner, H., Mueller, E. d., \& Murphy, D. L.. (1987). 


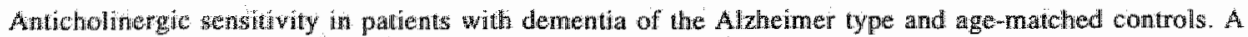

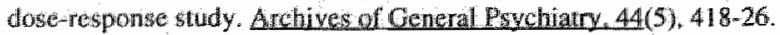

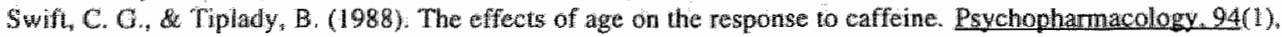
29.31 .

Saklo, M. Corhan, I, Diez-Roux, A. V. Chambless, L., Cooper, L., Folsom, A. R, Fried, L. P., Knopman, D. \& Wieto, J. J. (1996). Estrogen replacement therapy and cognitive functioning in the atherosclerotic risk in communties (ARIC) study, American Joumal of Bpideniology, 144(11), 1048-1057.

"1"arig, M.-X., Jacobs, D., Stern, Y., Marder, K., Schoheld, P., Gurland, B., Andrews, H., \& Mayeux, R. (1996). Ettect of egtrogen during munopause on risk and age at onset of Alzhemer's disease. The Lancet 348. (August 17h) $429-432$.

Tariol, P. N., Cohen, R. M, Welkowilz, I. A., Sunderland, T., Newhouse, P. A., Murphy, D. L., \& Weingartner, H. (1988a). Multipledose arecolin infusions in Alzheimer's disease. Arotwives of General Picychatry $45,901-995$.

Tulakuwa, K. (1971) Maintaining concentration (TAF) as a measure of mental stress. In K. Hashimoto, et al. (E) $\mathrm{s}$ ): Methodology in human fatigue Assessment. London: Taylor \& Prancis.

Terry, W.S. \& Phifer, B. (1986). Caffeine and memory performance on the AVLT. Joumal of Clinical Pyclioloyy. $42,860-863$.

Thayer, $\mathbb{R}, \mathrm{E}$, (1986). Activation-Deactivation Adjective Checklist: current owerview and structural analys is. Psychological Reporis. Apr: 58(2), 607-614.

Thomporowski, P. D., Ellis, N. R. (1986). Effects of exencise on cognitive processes: a review. Psychological Bulletin, $92,338-346$.

Tonporowski, P. D., Ellis, M. R, \& Stephens, R. (1987). The immediate effects of strenuous exercise on free recall memoty. Brgonomics, 30(1), $121-9$.

Torall Allerand, C. D., Miranda, R. C., Benthan, W. D., Sohrabji, F., Brown, T. J., Hochberg, R. B., \& MacLusky N. J. (1992). Estrogen receptors colocalize with low affinity nerve growth factor receptors in cholinergic neurons of the basal forebrain. Broceedings of the National Academy of Sciences of the United States of America, $89(10$, May), $4668-4672$.

Unrug-Neervoort, A., Luigtelaar van, G., \& Coenen, A, (1992). Cognition and vigilance: differential effects of diazepam and buspirone on memory and psychomotor performance. Biological Psychologyl Phamacopsychology, 26, 146.150.

Utian, W. H. \& Schiff, I. (1994). NAMSmGallup survey on women's knowledge, information sources, and attitudes to menopause and hormone replacement therapy. Menopause.

Van der Meer, C., \&anTilburg, W. (1983). Psychische Stoomissen bij Endocriene Ziekten. Alphen aan den Rijin/Brussel: Statleu.

Van Prag, H. M. (1996), Serotonin Related, Amxiety/Aggression Driven, Stressor Precipitated Depression: a Psycho Biological Hypothesis, Europen Psychigury, IL(2), 57.67.

Van Boxtel, M. P. J., Langerak, K., Houx, P. J., \& Jolles, J (1996). Self-reported physical activity, subjective thealth, and cognitive performance in older adults. Experimental Aging Research, 22(4), 363-379.

Vantulle, O. \& Dervol, R. (1976). A double blind study into the influences of estriol on a number of psychological tests in postmenopausal women. In: P.A. van Keep, R. B. Greenblatt, \& M. Albeaux Fernet (Eds.) Cohsensus on Menopause Research, (pp. 94-99). Lancaster: MTP Press.

Vanuect, R. C, \& Mujsce, D. J. (1992). Effect of glucose on perinatal hypoxic-issehemic brain damage Biol Neonate. $62(4), 215.24$.

Vehman, J. B. P. (1992). Hidden effects of moise nuevealed by cardiovascular analysis. Groningen: Universiny Press.

Vender, J. R. Nair, S. N., \& Lehmana, J. C. (1995). Pharmacotherapeutic potential for compounds acting at NMDA receptors: Update 1995. Expert Opin Invest Drugs, 4(5), 475-485.

Verhogeghen, $P_{\text {. }}$ Marcoeth, A \& Goossens L. (1993). Facts and Fiction About Memory Aging: A Quantitative Integration of Research Findings. Joumath of Gerontology, 48(4), P157-P171.

Wadworth. A. N., Chrisp, P. (1992). Co-dergocrine mesylate. A review of its pharmacodynamic and pharmacokinetics properties and therspeutic use in age-related cognitive decline. Drugs. and Aging 2 (3), 153 73.

Willin, A. \& Cotfries, C. G. (1990). Biochemical substrates in normal aging and Alzheimer's diseage. Phatmacopisychatry, 23, 37-43

Warburton, D. M. \& Brown, K. (1972). The facilltation of discrimination performance by physostigmine sulphate. PSychopharmalogia, 27, 275-284. 
Warburton. D.M. (1995) Effects of caffeine on cognition and mood without caffeine abstinence. Psychopharmacology, 119, 66-70.

Watters, P.A. \& Martin, Fo, Schreter, Z. (1997). Caffeine and cognitive performance: the nonlinear YerkesDodson Law. Human Psychopharmacology, 12, 249-257.

Webb, W. B., Levy, C. M. (1982). Age, sleep deprivation and performance. Psychophysiology 19(3), 272276.

Webb, W. B. (1985). A further analysis of age and sleep deprivation effects. Psychophysiology 22(2), 156-161.

Webb, W. B., Campbell, S. S. (1980). Awakenings and return to sleep in an older population. Sleepenas, $41-46$.

Webb, W. B. \&evy, C. M. (1984). Effects of spaced and repeated total sleep depriwaton. Ergonomics. 27. 45-58.

Weinachter, S. N., Blavet, N., ODonnell, R. A., MacKenzie, E. T., \& Rapin, J. R. (1990). Models of hypoxia and cerebral ischemia. Pharmaconsychiatry, 23, 94-7.

Weiss, B. L. (1987). Failure of nalmefene and estrogen to improve memory in Alzheimer's disease. American Iournal of Psychiatry, 144(3, March).

Wesensten, N. J., Crowley, J., Balkin, T., Kamimori, G., Iwanyk, E., Pearson, N., Devine, J., Belenky, G. \& Cymernan. A. (1993). Effects of simulated high altitude exposure on long-tatency event-related brain potentials and performance. Aviation. Space. and Environmental Medicine, 64(1), $30-6$.

Wesnes, K. A., Anand, R., Simpson, P. M., \& Christmas, L. (1990a). The use of a scopolamine model to study the potential nootropic effects of aniracetam and piracetam in healthy volunteers. Journal of Psychopharmacology, 4(4), 219-232.

Wesnes, K. A., Simpson, P., Christmas, L. \& Siegfried, K. (1990b, ) Effecis of HP029 in a scopolamine model of ageing and dementia. Paper presented at the 17 th Congress of C.I.N.P., Kyoto, Japan.

Wesnes, K., \& Warburton, D. M. (1983). Effects of scopolamine on stimulus sensitivity and response bias in a visual vigilance task. Neuropsychobiology $2(2-3), 154-157$.

Wesnes, K. A. Simpson, P. M., Christmas, L. Anand, R., \& McClelland, G. R. (1989). The effects of moclobemide on cognition. LNeural Transm Suppl, 28(91), 91-102.

Wesnes, K., Simpson , P. M., \& Kidd, A. (1987). The use of a scopolamine model to study the nootropic effects of tenilsetam (CAS 997) in man. Medical Science Research Psychology and Psychialty. 15 (17-20), 1063-1064.

Wesnes, K., \& Revell, A. (1984). The separate and combined effects of scopolamine and ricotine on human intormation processing. Psychopharmacology. 84(1), 5-11.

Wesnes, K. (1996, 18-20th September). The cognitive, subjective and physical effects of gincosaro in healihy volunteers with neurasthenic complaints. Paper presented at the Annual Meeting of the Psychobiology Section of the British Psychological Association, Langdale: England.

WickJund, I., Karlberg, J., \& Mattson, L.-A. (1993). Quality of life of postmenopausal women on a regimen of transdermal estradiol therapy. American Journal of Obsterrics and Gynecology, 168(3, Part 1), 824-830.

Wilford, G. K.. \& Harrold, P. L. (1996). Treatment of Alzheimer"s disease: future directions. Acta Neurologicat Scandinavica 165 (Supplement), 128-136.

Wilkinson, R. T. (1990). Response-stimulus interval in choice serial reaction time: interaction with sleep deprivation, choice and practice. The Quarterly Journal of Experimental Psychology $42 \mathrm{~A}(2), 401-423$.

Winer, B. J. (1971). Statistical principles in experimental design. New York: McGraw Hill.

Witteman, J. C. M., Grobbee, D. E., Kok, F. J., Hofman, A. \& Valkenturg, H. A. (1989). Increased risk of atherosclerosis in women after the menopause. British Medical Journal. 298, 642-644.

Wong. M. \& Moss, R. L. (1992). Long-term and short-term electrophysiological of estrogens on the synaptic properties of hippocampal $C A$ I neurons Journal of Neuroscience $12.3217-3225$.

Woodruff-Pak, D. S., Finkbiner, R. G. (1995). Larger nondeclarative than declarative deficits in learning and memory in human aging. Psychology and Aging. 10(3), 416-426.

Woolley, C. \& McEwen, B. S. (1993). Roles of estradiol and progestrone in regulation of hippocampal dendritic spine density during cestrus cycles in the rat. Journal of Comparalive Neurology. 336, 293-306.

Woolley, J. C. M., Gould, E., Frankfurt, M., \& McEwen, B. S. (1990). Naturally occurring fluctuation in dendritic spine density on adult hippocampal pyramidal neurons. The Joumat of Neuroscience 10(12), 4035 4039.

Yates . C. M., Buterworth, J., Tennant, M. C.. \& Gordon, A. (1990). Enzyme activities in relationship to pH and lactate in postmortem brain in Alzheimer-type and other dementias. Journal of Neurochemistry. $55(5)$. 
Yerkes, R. M., Dodson, I, D. (1908). The relation of strength of stimuli to rapidity of habituation. Journa: of Comparative Weurology and Psychology. $18,459-482$.

Young, S. N, Smith, S. E., P.hl, R. O. \& Erwin, F. R. (1985). Tryptophan depletion causes a rapid lowering of mood in normal males. Bsychopharmacology Berl, 87(2), 173-7.

Yu, G. Maskray. V., Jackson, SHD., Swift, CG. \& Tiplady, B. (1991). A comparison of the cemtral nervous system effects of caffeine and theophylli in ellerly subjects. British Journal of Clinical Pharmacology $32,341-345$.

Zjilstra, $F_{*}$ \& Meijman, $\mathrm{T}$ (1989) Het meten van mentale inspanning met behulp wan een subjectieve methode. In: T. Meijman (Eds). Mentale belasting en werkstress: een arbeidspsychologische benadering. Assen/Maastricht Van Gorcum pp.42-61.

Zyhtarz, G., Saletu, B., Hitzenberger, G., Grinberger, J., Anderen, P., Linzmayer, L., \& Rameis, H. (1994). Double-Blind, Placebo-Comtrolled; Pharmacokinetics and-Dymamic Studies with Two New Formulations of Piracetari (finfusion and Syrup) Under Hypoxia in Man. Neuropsychopharmacology. 10(3S Pt 2), 117S. 


\section{Summary}

This thesis is concerned with age-related cognitive deficiencies and the evaluation of possible relevant methods to investigate the effect of putative cognition enhancers. The thesis is divided in three parts. Part one describes experimental studies into models of simultaneously cognitive deficiency. Part two describes studies into the effect of caffeine in models of cognitive deficiency. Part three addresses the effects of Hormonal Replacement Therapy on simultaneonsly cognitive deficiency as an example of the clinical approach in testing cognition enhancers in nondemented elderly.

\section{Part One}

In part one, the problem is addressed what comprises age-related cognitive decline and how it is measured and modelled. Chapter 2 is entirely devoted to this problem, whereas in chapters 3 and 4 , the results of empirical studies are presented which are characterized by the search for new specific and sensitive indices of age-related cognitive decline. The study in chapter 3 is based on an epidemiological study, whereas the study in chapter 4 is based on an experimental design. The studies reported in chapters 5 and 6 are evaluations of experimentally induced cogniave dysfunctions, which resemble aspects of age-related cognitive decline.

In chapter 2, a review is presented of the age-related physiological and psychometric changes. The impaired manipulation and organization of information in short term memory in elderly subjects and the slowing of complex task performance has lead to the conclusion that aging is associated with a decrease in information processing capacity or mental resources. The agerelated decline of brain reserve capacity is put forward as an explanatory concept, reflected by the accumulated acquisition of health risk factors or biological life events "age-related decrease of cerebral glucose consumption, and the age-related functional decline of severall neurotransmitter systems in the brain. By using an experimental model it is possible to test putative cognition enhancing drugs in healthy wolunteers according to a placebo-controlled, double-blind design. The model tests whether these drugs are able to reverse the experimentally induced age-related cognitive dysfunction. Human models to simulate and hence to experimentally study these phenomena are scarce when compared to the amount of studies employing animal models. In chapter two, several human pharmacological, physiological and behavioral models of age-related cognitive dysfunction are critically evaluated. The following human models of cognitive dysfunction are described in the chapter: the hypoxia and hyperventilation models of brain hypoxia; the neurotransmitter models of memory dysfunction by means of cholinergic blockade, benzodiazepines and serotonergic depletion; and the functional behavioral models of fatigue: sleep deprivation and physical exhaustion. In a separate section on putative new models, experimentally induced hypoglycemia, catecholamine depletion and partial sleep deprivation are proposed as new experimental procedures to model aspects of age-related cognitive dysfunction. Of each model, a theoretical background is given, followed by a description of the cognitive effects of the induction. Subsequently, studies are described in which the model is used to demonstrate the efficacy of putative cognition enhancing substances. Each paragraph ends with a short discussion describing the state of the art. It was concluded that some of the models are adequate to simulate age-related cognitive decline but not dementia. Furthermore, young subjects may overcome some of the experimentally induced cognitive dysfunction by investing more effort. Motivational and expectancy factors could then confound results. Middle-aged healthy 
wolunteers may be better suited ass subjects in model studies of age-related cognitive decline. This group can be expected to have more difficulty to overcome an induced cognitive deficiency, since dectements in cognitive function are already seen in the fourth and fifth decade. Furthermore, it was discussed that the specificity of the models is difficult to maintain, as the different neurotransmitter systems interact and therefore experimentally induced cognitive dysfunctions induced through one neurotransmitter pathway, may be reversed or attenuated through another, indirectly related, neurotransmitter pathway. It is therefore proposed that the siunultaneous use of different (neurotransmitter) models in one experimental design may illuminate through which pathway a putative new drug is most likely to exert its main effects on cognitive function. However, many models or combination models have not been tested thoroughly and still await evaluation.

In chapter 3, age-related cognive dysfunction was investigated in more detail. This chapter describes the effect of age on the performance of a complex word-learning lest with and without distraction. Four groups, stratified for age $(24-36,37-49,50-62$, and $65-77$ years), sex and level of occupational achievement, comprising a total of 111 subjects, were included. The mediating effect of education, health, and speed of processing on memory performance was assessed by using regression analyses. The results showed interactions between age and learning and between atge and rate of forgetting for the word-learning test with distraction, but not for the word-learning test without distraction. Slowness and a decrease in subjective health explained the age-related variance in performance of both memory tests. In chapter 4 , the age-related impairment was further investigated, using a complex word learning test with distraction. Sixty healthy subjects of both sexes, equally divided over three age groups (26-34, 46-54 and 65-75 years), matched for level of occupational achievement, were recruited by means of an advertisement. There was no interaction between age and learning or age and rate of forgetting. Hence, in a healthy population, there is no additional age-related impairment of performance of a complex memory task. The effect of basic sensorimotor speed on recall was mediated by working memory storage capacity. The variance in performance of the delayed recall task was explained by working memory capacity and memory search functions, which partialled out the effect of age.

In chapter 5 , a study is described in which experimentally induced mild hypoxia and hypocapnic hyperventilation were evaluated as models of cognitive deficiency. Twenty healthy male volunteers (aged 23-30) were tested according to an order balanced, single blind, controlled cross-over design. Subjects received hypoxic air $\left(13.6 \% \mathrm{O}_{2}\right)$ or normal air $(21 \%$, normoxia) through a mask as a control. One week later, they underwent hypocapnic (end tidal $\mathrm{CO}_{2}<2.5$ $\mathrm{kPa}$ ) or normocapnic hyperventilation ( $\pm 5 \mathrm{kPa}$ ). No treatment effects were found on memory tasks. Mild hypoxia showed a decrease in visual sensitivity and an increase in the reaction time (R'T) of the incompatible choice RT task as compared to normoxia. The impairing effect of hypocapnic hyperventilation on psychomotor speed could be attributed to the effects of overbreathing (normocapnic hyperventilation). In addition, the wearing of a mask (normoxia) also impaired performance of the choice RT task and of sensorimotor processing speed. It was concluded that studies that have not used control treatments in either of the two models should be regarded with caution.

In chapter 6 , the results are described of a study into the effect of prolonged physical exercise on cognitive performance. Fifteen male endurance-trained athletes (aged 18 to 42 years) performed a one-hour, bicycle ergometer endurance test at $75 \%$ of their maximal work capacity (Wmax). Psychomotor and cognitive tests were administered before and immediately after exercise. These 
consisted of simple reaction time (RT), 3-choice $\mathbb{R T}$ and Stimulus-Response (S-R) incompatible $\mathbb{R T}$ tasks, a finger tapping task, and the Stroop test. Simple RT tasks, but also the S-R incompatible RT and the Color Word Interference in the Stroop test sthowed an increase in speed of performance after exercise compared to baseline. An enhanced activation was probably responsible for this better performance on psychomotor and cognitive tests. Since performance in the most complex task, the Interference subtest of the Stroop, was also improved after exercise, the expectancy of the subjects of a potential positive effect of exercise may have been responsible.

\section{Part Two}

This part of the thesis contains three studies in which the putative cognition enhancing effects of caffeine are tested. The first, described in chapter 7 , is a study into the effect of caffeine on agerelated cognitive decline. The other two, described in chapters 8 and 9 are applications of models of age-related cognitive deficiency in which the effect of caffeine on cognitive penformance is studied according to a placebo-controlled, double-blind experimental design.

In chapter 7 , the results are described of a study in which the effect of caffeine $(225 \mathrm{mg})$ on cognitive performance, as a function of age, is evaluated. The study was conducted in young; middle-aged, and old subjects according to a placebo-controlled parallel groups design $(n=60)$. Groups were matched for lewel of education and sex. Positive effects of caffeine, as compared to placebo, were found in middle-aged subjects in the first trial of the word learning test. In contrast, caffeine had negative effects on the speed of searching short- term memory in young subjects. Caffeine had no effect on the intercept, which is an indicator for sensorimotor speed, of a memory scanning task. The middle-aged subjects appeared to regullarly consume twice as much caffeine as the young and old subjects. These results were similar to earlier findings in a large population study. Although statistical analyses with habitual caffeine consumption as a covariate did not yield different results, a caffeine withdrawal effect was hypothesized to be responsible for the reduced cognitive performance of middle-aged subjects receiving placebo. The habitual use of large amounts of caffeine by middle-aged subjects may be a means to overcome the agerelated decrease in cognitive functioning that is caused by changes in information processing.

In chapter 8 , a study is described, in which the effects of caffeine on cognitive performance are evaluated in two models of experimentally induced cognitive dysfunction. Recently, caffeine was. reported to attenuate the scopolamine induced cognitive dysfumction. In this chapter the effects of caffeine on the cognitive dysfunction induced by hypoxia and hyperventilation are reported. Twenty healthy male college students (aged 23-30) were tested according to a mixed, double blind, placebo-controlled cross-ower design. Caffeine $(230 \mathrm{mg})$ or placebo was administered according to a between subjects design. On one day, subjects received hypoxic air $\left(13.6 \% \mathrm{O}_{2}\right)$ and normal air ( $21 \% \mathrm{O}_{2}$, normoxia) through a mask as a control. On another day, with one week in between, they hyperventilated (end tidal $\mathrm{CO}_{2}<2.5 \mathrm{kPa}$ ) and hyperventilated with additional $\mathrm{CO}_{2}$ (normocapnia) until normal end tidal levels were attained ( $\pm 5 \mathrm{kPa}$ ). During all inductions and baselines subjects performed cognitive tasks, including a working menory and a long term memory task, and a task measuring visual perceptual sensitivity. No effect of inductionis were seen on memory functions. Hence, hyperventilation was not shown to be a better model ats compared to mild hypoxia. Caffeine appeared to impair working and long term memory functions. probably through enhancing arousal to a level which was detrimental to task performance. In addition, although hypoxia decreased the visual perceptual sensitivity on the signal detection task, no effect was seen of caffeine on this task. It was concluded that caffeine was not able to reverse 
the cognitive dysfunction induced by hypoxia. Furthermore, different models of cognitive dysffunction, such ass scopolamine and hypoxia, can be used simultaneously to observe the profile and mechanism of action of potential new cognition enhancers.

In chapter 9 a study is reported into the effects of three carbohydrate electrolyte solutions (CES) containing different amoants of caffeine on cognitive function. The combined effects of these drinks and exercise on cognitive functions were investigated according to a placebo-controlled, double-blivid, crossover design. On five separate occasions "ffteen endurance trained male athletes (23.3 years) received water placebo. CES placebo $(68.8 \mathrm{~g} / \mathrm{L})$, and three CES drinks containing low, medium and high dosiages of caffeine $(150,225$, and $320 \mathrm{mg} / \mathrm{L})$. The drinks were consumed before an all out 1 hour time trial on a bicycle ergometer $(8 \mathrm{ml} / \mathrm{kg}$ ) and during exercise $(6$ $\mathrm{ml} / \mathrm{kg}$ ). Psychomotor and cognitive tasks, long-term memory, perceptual interference, visual signal-detection, and choice reaction time, were carried out immediately before and immediately after exercise. Before exercise, long-term memory was improved by CES plus low dose caffeine when compared to both placebos. Immediately after exercise, cognitive performance was improved by CES plus low- and medium-dose caffeine compared to both placebos. The cognition-enhancing effects of caffeine could not be explained by vigilance-enhancing effects before exercise or restoration of deteriorated psychomotor and cognitive performance due to fatigue after exercise. These results comprise the first practical demonstration of the cognition enhancing effects of addition of low amounts of caffeine in CES before and after strenuous physical exercise.

\section{Part Three}

In part three, the nature of the effect of hormonal replacement therapy on age-related cognitive decline is investigated. A review is presented in chapter 10 , in which the effects of Hormonal Repllacement Therapy (HRT) in elderly and demented women are critically discussed. In chapters 11 and 12, the results of empirical studies are presented which are characterized by the question if HRT has cognition enhancing effects. The study in chapter 11 is based on an epidemiological study, whereas the study in chapter 12 is based on an experimental design.

In chapter 10, a literature review of studies into the cognitive effects of HRT is presented. The review is guided by three main questions with regard to the relationship between HRT and cognitive function. The first is concerned with the possible long term protective effect of HRT. Second, it is unclear wherher HRT exerts its effects specifically on verbal memory, or is reflected by a global enhancement of cognition, as expressed by improved sensorimotor speed increased subjective activation and enhanced feelings of well-being and whether this effect is clinically relevant. The third question is concened with the mechanism of action of HRT combination therapy. These issues are considered separately as to Alzheimer's dementia, vascular dementia, age-associated cognitive decline and normal post-menopausal women. Several methodological issues emerged which hampered straightforward answers to the abovementioned questions. These included several sources of bias or confounding in the patient studies, such as: sampling errors, different methods to diagnose dementia and age-related cognitive decline, self-report as to assess MRT-use and length of use, extreme variation as to the duration of HRT-use, differences in educational level and socio-economic status between HRT-users and controls, inclusion of male subjects, small sample sizes, lack of a blinded control condition and a short duration of treatment. The data of the population based studies suggested that effects of HRT were either very specific (function or age-group) ol absent. In the experimental studies, methodological differences may explain part of the contrasting results found. These problems concern: the number of subjects, 
insensitivity of designs and tests, non-compliance, lack of a blinded control condition, difficulties in comparing different HRT preparations and differences between studies as to the timepoints of assessment. In general, the findings were considered to be suggestive of global effects of BRT on cognitive functions. Neurotransmitter systems that may be implicated in cognitive aging and dementia and that have been found to be positively infuenced by estrogens are the cholinergic and the monoaminergic neurotransmittersystems. Estrogens have been repotted to increase levels of neurotransmitters, through their influence upon the availability of precursors, the activity of enzymes needed for synthesis and the degradation of neurotransmitters. Estrogens have also been reported to act upon receptors and may even affect the structural hardware of the braili, which can explain their protective effect, especially in Alzheimer's disease and in the long term effects of aging. Most effects of estrogens are opposed by progestagens, such as affects on the noradrenergic systems, the dendritic outgrowth, and potential cerebrowascular effects. Cholinergic, serotonergic, and the potentially protective (i.e. antioxidant) actions of estrogens are probably unopposed by progestagens. It was concluded that it is plausible that estrogens have protective and enhancing effects on cognitive functions in aging and mild to moderate dementia and may exert their protective and enhancing effects via an action upon many biological levels. However, evidence for protective effects in the normal population is too inadequate to call for a general hormonal substitution therapy for all women.

In chapter 11 , the results are presented from a large cross sectional study into the association of Hormonal Replacement Therapy with cognitive performance, in middle-aged women. Estrogen Replacement Therapy (ERT) in postmenopausal women could be a protective factor against Age related Cognitive Decline (ARCD) and Alzheimer's Disease (AD). The rationale of the present study was to evaluate whether ERT-use is associated, in a positive manner, with cognitive functioning in middle-aged women. The study was performed as a separate analysis within the Maastricht Aging Study (MAAS), where 1869 subjects aged 24 through 82 were investigated both medically and neuropsychologically. Twenty-three ERT-users were found in the age range of 4565 years. A case-control design was used where these subjects were compared to 319 controls from the same age range. Statistical analyses were performed to assess differences between estrogen-users and controls on socio-demographic-, health risk-, perceived health- and cognitive function variables. Furthermore, the independent and the possible confounding influence of age, education, health risk factors- and perceived health variables on ERT-use and its association with cognitive performance respectively, were determined. Unexpectedly, ERT users had more subjective complaints of memory dysfunction as well as of anxiety and depression. No effects of ERT were found on objective memory performance or cognitive flexibility. Although ERT-users had more years of education, a independent trend towards a positive relationship between ERT-use and sensorimotor speed was detected. The small proportion of ERT-use in the MAAS database is probably representative for the Netherlands. Furthermore, it should be kept in mind that about half of these women had undergone hysterectomy and/or ovariectomy, which may explain why this group overall had more psychological complaints. As far as the explorative nature of the analysis permits, the results showed a trend in the direction of a general activating effect of ERT-use, rather than a specific association between ERT-use and memory or cognitive performance.

In chapter 12, the results are described of a follow-up study into the effects of combined HRT on cognitive function in postmenopausal women. This study was carried out to investigate long term effects of the combination Hormonal Replacement Therapy (HRT: estrogens and progestagens) 
in postmenopausal women. Subjects were tested during the estrogen-progestagen phase of the cycle. A carefully matched group of post-menopiausal women who did not receive therapy was used as a control group ( $n=11$ ). The monthly withdrawal bleeding in the HRT group ( $n=11$ ) precluded the use of a placebo-treated control group. Long term memory functions were seen to be enhanced with the combined use of estrogens and progestagens after 6 and 12 months. Also, both subjectively experienced activation and the general feeling of well being was improved in HRT users as compared to controls as measured with the AD-ACL and the Neurovegetative questionnaires. It was concluded that combined HRT had a positive long term effects on both memory and a general feeling of well being.

\section{Conclusilion}

In conclusion, this thesis has shown that several relevant methods exist which do not involve the target population for treatment with cognition enhancers (i.e. the elderly subjects with objective cognitive impairment). Models of age related cognitive decline (scopolamine, mild hypoxia, and physical exhaustion) can be of walue in testing potential cognition enhancers. In addition, evaluation of drug effects in large cross sectional samples of otherwise healthy subjects (in this thesis the MAastricht Aging Study) may prove a relewant source of information. 


\section{Samenvatting}

Het centrale thema van dit proefschrift betreft leeftijds-gerelateerde cognitieve dysfuncties an de evaluatie van relevante methoden om de werking van cognitie-verbeterende stoffen te kumen testen. Het proefschrift bestaat uit in 3 delen. Deel 1 beschrijft experimentele studies van modellen van cognitieve deficientie. Deel 2 betreft het effect van caffeine als ijkstof in modellen van cognitieve dysfunctie. Deel 3 behandelt de effecten van hormonale substitutie therapie op leeftijdsgerelateerde cognitieve dysfunctie als een voorbeeld wan klinisch onderzoek waarbij cognitie verbeterende stoffen bij niet demente subjecten worden getest.

\section{Deell 1}

In deel 1 bespreken we wat leeftijdsgerelateerde cognitieve achteruitgang inhoudt en hoe we het kunnen meten en modelleren. In hoofdstuk 2 wordt dit onderwerp in zijn geheel besproken. terwijl in hoofdstukken 3 en 4 de resultaten van empirische onderzoek naar specifieke en sensitieve indicatoren voor leeftijdsgerelateerde cognitieve achteruitgang worden gepresenteerd. Hoofdstuk 3 is gebaseerd op een epidemiologische studie, terwijl hoofdstuk 4 gebaseerd is op een experimenteel design. Hoofdstukken 5 en 6 betreffen evaluaties vam experimenteel geinduceerde cognitieve dysfuncties.

Hoofdstuk 2 begint met een overzicht van de fysiologische en psychometrische veranderingen die bij veroudering plaatshebben. Ouderen worden verondersteld om met name moeite te hebben met de uitvoer van complexe cognitieve testen ten gevolge van een vermindering van de mentale reserve capaciteit. Deze kunnen we fysiologisch waarnemen in structurele (afname neuronale verbindingen) en functionele veranderingen (verminderde cerebrale doorbloeding en neurotransmitter synthese) die plaatshebben bij veroudering.

Modellen van verouderings-gerelateerde cognitieve deficiëntie richten zich met name op het simuleren van de functionele verouderingsgerelateerde veranderingen bij gezonde vrijwilligers. Cognitie-verbeterende stoffen zouden, volgens hun definitie, deze deficiënties moeten kunnen opheffen. In hoofdstuk 2 wordit een overzicht gepresenteerd waarin verschillende modellen van leeftijds-gerelateerde cognitieve achteruitgang worden geëvalueerd; zoals het hypoxie en hyperventilatie model van de cognitieve effecten ten gevolge van een verminderde doorbloeding en verminderde zuurstoftoevoer; de neurotransmitter modellen van geheugendysfuncties, zoals het cholinerge blokkade (scopolamine) model, het benzodiazepine model en het serotomine deplette model; en de functionele gedragsmodellen zoals het model van slaapdeprivatie en het model van fysieke atputting. Tevens is een deel gewijd aan potentiele nieuwe modellen, zoals hel model van experimenteel geïnduceerde hypoglycemie; de catecholamine depletie modellen en het model van partiêle slaap-deprivatie. Bij elk model wordt een theoretische achtergrond gegeven, welke wordt gevolgd door een beschrijving van de cognitieve effecten van het model. Er worden tevens studies gepresenteerd om an te geven in hoeverre het model effectief is gebleken om potentiële cognitie verbeterende stofien te onderzoeken.

Er werd geconcludeerd dat een aantal van deze modellen wel adequat zijn om leeftijdsgerelateerde cognitieve achteruitgang te simuleren, maar niet dementie. Een problem bij sommige modellen is dat jonge mensen de cognitieve dysfuncties kunnen opheffen door zich meer in te spannen. Misschien zouden beter mensen van middelbare leeftijd kunnen worden geincludeerd als proefpersoon. We verwachten dat deze groep meer moeite heeft om de geïnduceerde cognitieve dysfuncties op te heffen, omdat we al een cognitieve achtertitgang. 
kunnen watmemen in het vierde en vijfde decenum van heit leven. Daamaast blijkt dat de meeste modellen weinig specifieke effecten hebben, omdat de verschillende neurotransmittersystemen interacteren. Door verschillende modellen te gebruiken kan worden onderzocht via welke weg een middel effecten uitoefent op cognitieve functies. Echter, andere potentiëlte neurotransmittermodellen (bijw. het serotonerge depletie model) of combinatie modellen zijn onvoldoende getest en moeten nog worden geevalueerd met betrekking tot de mogelijke bruikbaarheid.

In hoofdstakken 3 en 4 werd onderzocht welke cognitieve testen met name gevoelig zijn voor veroudering. In hoofdstak 3 werd een onderzoek gepresenteerd van een groep aselect getrokken proefpersonen $(n=111$ ) van de leeftijd 25 tot 77 jaar afkomstig uit de MAastricht Aging Study (MAAS). Vier leeftijdsgroepen $(24-36,37-49,50-62$, en 65-77 jaar) gematched voor sexe en beroepen/opleidingsniveau werden onderzocht. De prestatie (leervermogen en snelheid van vergeten)op een complexe geheugentaak met distractoren bleek extra verlaagd als gevolg van leeftijd. Dit effect zagen we niet bij de reguliere geheugentaak. De complexe taak werd dus verondersteld extra gevoelig te zijn woor leeftijds-gerelateerde achteruitgang. De cognitieve vertraging en de subjectief ervaren gezondheid (maar niet de objectieve gezondheidsstatus) kon in dit onderzoek de leeftijds-gerelateerde variantie verklaren, zoals werd onderzocht met regressie analytische technieken. In een ander onderzoek (hoofdstuk 4) werd de prestatie op de complexe taak verder onderzocht bij gezonde proefpersonen (zowel objectief als subjectief ervaren gezondheid). Zestig proefpersonen, die zich hadden aangemeld via een advertentie, werden verdeeld over 3 leeftijdsgroepen $(26-34,46-54$ en $65-75$ jaar) en gematched voor sexe en beroepen/opleidingsniveau. Opnieuw vonden we de leeftijds-gerelateerde verminderde retentie van informatie bij de complexe geheugentakak met distractoren. Daarnaast vonden we een vertraging van de reactietijden op taken ten gevolge van veroudering. Verder bleek uit het onderzoek dat de variantie in de prestatie op de uitgestelde herinneringstaak werd verklaard door de opslagcapaciteit en de zoeksnelheid wan het werkgeheugen. Echter, gezonde (objectief en subjectief ervaren) proefpersonen laten geen extra leeftijds-gerelateerde achteruitgang zien op een complexe geheugentaak. In de daarop volgende experimenten is een verouderingsgevoelige testbatterij gebruikt welke bestond uit de complexe geheugentaak met distractoren en verschillende reactietijd-taken on de snelheid van informatieverwerking te kunnen meten.

In de hoofdstukken 5 en 6 worden twee nieuwe modellen van leeftijdsgerelateerde cognitieve achteruitgang geëvalueerd. Experimenteel geïnduceerde milde hypoxie (zuurstoftekort) is een model dat momenteel wordt gebruikt om de effecten van potentiele cognitie-verbeterende sioffen te liesten. Er is gesteld dat hypocapnische hyperventilatie een mogelijk nieuw model zou zijn om de leeftijd-gerelateerde relatieve zurstofdeficiëntie en de hieruit voortvloeiende cognitieve dysfunctie te simuleren. In hoofdstuk 5 werden de effecten van het hypoxie en hyperventiatie model onderzocht bij gezonde, jonge (23-30 jaar), mannelijke vrijwilligers door middel van een voot" volgorde gebalanceerd, enkel-blind, gecontroleerd cross-over design. Hierbij onderzochten we het effect van hypocapnische hyperventilatie $\left(\mathrm{CO}_{2}\right.$ in witgeademde luch $\left.<2.5 \mathrm{kPa}\right)$, wan milde hypoxie $\left(13.6 \% \mathrm{O}_{2}\right.$ in ingeademende lucht), en van de controle condities, het effect van het dragen van een masker (normoxie $21 \% \mathrm{O}_{2}$ in ingeademende lucht) en het effect van overademenen sec (nomocapnische hyperventillatie), waarbij extra $\mathrm{CO}_{2}$ werd toegevoegd aan de ingeademde lucht (tot $\pm 5 \mathrm{kPa}$ in uitgeademde lucht). Hoewel wij geen geheugen-dysfuncties vonden ten gevolge van milde hypoxie, zagen we well een vertraging ten gevolge van hypoxie op complexe taken welke wisuele detectie en respons-inhibitie vereisten, als vergeleken met de controle conditie, normoxie. Verder bleek hypocapnische hyperventilatie niet een beter model te 
zijn dan het hypoxie model. De vertraging op taken die wel optrad tijdens hyperwentilatie kon worden toegeschreven an het effect van overademen (nomocapnische hyperventillatie) en aar het dragen van een masker. Dat betekent dat de resulaten van dergelike studies die geen controle conditie hebben ingebouwd voorzichtig moeten worden geinterpreteend.

In hoofdstuk 6 worden de resultaten beschreven van een onderzoek naar de cognitieve effecten van fysieke uitputting als een mogelijk model van asthenie. Vijftien duurgetrande, mannelijke atleten (18-42 jaar) werden geïncludeerd. De inspanning bestond uit een tijdrit op de fiets ergometer, waarbij ongeveer 60 minuten op $75 \%$ van het maximaal vermogen moest worden gefietst. Proefpersonen hadden een snellere prestatie op een aantal taken (Stroop. Simpele en Complexe RT) na de uitputtende inspanning. Mogelijk was de verhoogde fysiologische activatie verantwoordelijk voor dit effect. Aangezien de prestatie van de meest complexe taak (de interferentie subtest van de Stroop) ook was verbeterd na inspianning, kunnen motivationele factoren en de verwachtingen omtrent de experimentele conditie de onderzoeksresultaten hebben beinvloed.

\section{Deel 2}

In het tweede deel van dit proefschrift wordt het effect van cafeine als ijkstof bij modellen van cognitieve deficientie besproken. Eerder is namelijk gesteld dat het effect wan een cognitieverbeterende stof minstens sterker zou moeten zijn dan het effect van cafeine. In hoofdstuk 7 wordt een studie gepresenteerd natr de effecten van cafë̈ne op leeftijds-gerelateerde cognitieve achteruitgang. In hoofdstuk $8 \mathrm{en} 9$ wordt het effect van cafeïne onderzocht in modellen wan leeftijdsgerelateerde cognitieve dysfuncties door middel van een placebo-gecontroleerd, dubbel blind, experimenteel design

In hoofdsiuk 7 worden de resultaten beschreven van het effect van $225 \mathrm{mg}$ ( 13 kopjes) cafeine bij gezonde jonge, middelbare en oude proefpersonen $(n=60)$ gematched voor opleiding en sexe. Het effect is onderzocht door midel van een placebo gecontroleerd, parallele groepen design. Cafeïne verbeterde werkgeheugenfuncties bij proefpersonen van middelbare leeftijd, maar niet bij jongeren of ouderen. Cafeine had negatieve effecten op de werkgeheugenfuncties bij jongeren. Geen effect werd gevonden op de sensori-motorische snellheid. Uit het huidige en eerder onderzoek is gebleken dat mensen van middelbare leeftijd gemiddeld meer cafeine consumeren dan mensen yan andere feeftijdsgroepen. Hoewel statische analyse met cafeine consumptie als covariaat geen verandering in de resultaten liet zien, zou het mogelijk kunnen zijn dat een ontrekkingsmeffect verantwoordelijk was voor de verminderde prestatie ten gevolge van consumptie van een placebo (decan bij proefpersonen van middelbare leeftijd. Mogelijk is het verhoogde cafeine gebruik bij mensen van middelbare leeftijd een compensatie voor leeftijdsgerelateerde cognitieve dysfuncties.

Er is verder gesuggereerd dat cafeine dosis.afhankelijke effecten heef. In hoofdstuk 8 bleek dat een vergelijkbare dosering van $230 \mathrm{mg}$ cafeüne opnieuw negatieve effecten had op het functioneren wan het werkgeheugen bij jongeren. Uit hoofdstuk 9 bleek bovendien dat $90 \mathrm{mg}$ ( 1 kopje) cafeine voor inspanning bij jonge proefpersonen (atleten) positieve effecten had op de geheugenfuncties. Deze bevindingen zijn vergelijkbaar met resultaten van eerdere onderzoeken.

In hoofdstuk 8 werd het effect van cafeine in het hypoxie en hyperventilatie model onderzocht. Twintig gezonde, mannelijke studenten ( 23 tot 30 jaar) werden onderzocht door middel wan een gemengd dubbel-blind, placebo gecontroleerd, cross-over design. Hoewel eerder $250 \mathrm{mg}$ cafieine de geheugendysfuncties ten gevolge van scopolamine (een model voor verouderingsgerelateerde 
cognitieve stoomissen) kon opheffen, had $230 \mathrm{mg}$ cafeine negatieve effecten op de lange termin getheugenfuncties na het dragen van aen masker, na hypoxie en na hyperventilatie. Er werd bovendien geen effect van cafeine gezien op de door hypoxie verminderde visuale sensitiviteit. Mogelijk voelden proefpersonen zich niet prettig tijdens de inductie van hypoxie, bij het dragen van een masker en tijdens het hyperventileren en interpreteenden zij hun toestand als bedreigend (pijn, gevoel te stikken, claustrofobie). $\mathrm{Er}$ is dan sprake van een negatieve activatie of distress (angst). De additionele verhoging van arousal door cafeine kan dan tot een slechte takprestatie leiden op de lange termijn geheugentak. Dit komt overeen met bevindingen van andere onderzoekers dat stress negatieve effecten kan hebben op het terughalen van informatie uit het lange termijn geheugen. Cafeine kon dus niet de door hypoxie geinduceerde cognitieve dysfuncties omkeren. Door verschillende modellen wan cognitieve dysfunctie te gebruiken kan het testprofiel en het werkingsmechanisme van mogelijk nieuwe cognitie-verbeterende stoffen worden onderzoeht.

In het model van fysieke uitputting (hoofdstuk 9) bleken lage en gemiddelde doseringen cafeine in een koolthydraten-bevattende drank een cognitie-verbeterend effect te hebben boven op het positieve effect dat fysieke inspanning had. Kennelijk heeft cafeine positieve effecten als proefpersonen (atleten) een positieve verwachting hebben ten aanzien wan de inductie. In de algemene discussie (hoofdstuk 13) wordt een alternatieve hypothese besproken welke er van uit gaat dat er bepaalde neurotransmitter-deficienties optreden na inspannende uitputting, welke cafeine zou kunnen hebben opgehewen.

\section{Deel 3}

Een aantal onderzoekers heeft gesteld dat modellen wan leeftijdsgerelateerde cognitieve achteruitgang niet kunnen dienen als test van cognitie-verbeterende stoffen omdat deze stoffen zich zouden moeten richten op de bescherming en instandhouding van fysiologisclie systemen.

In deel 3 wondt het effect besproken van hormonale substitutie therapie (HST) bij verouderingsgerelateerde cognitieve dysfunctie. In hoofdstuk 10 worden de effecten van HST bij oudere gezonde en demente vrouwen kritisch besproken in een overzichtsartikel. In hoofdstukken 11 en 12 worden de resultaten wan empirisch onderzoek besproken. Hoofdstuk 11 is gebaseerd op een epidemiologisch onderzoek, terwijl hoofdstuk 12 is gebaseerd op een experimenteel design.

In hoofdstuk 10 wordi cen literatuur overzicht van de cognitieve effecten van HST gepresenteerd. Hoewel epidemiologische onderzoeken laten zien dat het risico op Alzheimer's dementie verlangd is bij het gebruik van HST betekent dit niet per se dat er sprake is van een causale relatie. Op de experimenten met demente vrouwen an HST is vaak op methodologisch gebied veel aan te merken (kleine groepen, korte duur). Daarnaast bleek uit een longitudinale studie dat HST geen rol speelt bij het tegengaan van leeftijdsgerelateerde cognitieve achteruitang. Een reden voor het verschill in resultaten zou kunnen liggen in de diversiteit vam de gebruikte hormonale preparaten (diverse soorten oestrogenen met en zonder progestagenen). Van progestagenen is bijwoorbeeld bekend dat ze een aantal effecten van oestrogenen kunnen omkeren. Met name de lange termijn effecten van gecombineerde HST (progestagenen en oestrogenen) zijn onvoldoende onderzocht.

In hoofdsikk 12 werd een onderzoek beschreven naar de lange termijn effecten van gcombineerde HST bij gezonde post-menopansale vrouwen van middelbare leefijd door middel van een parallele groepen design. Uit deze studie bleek dat de gecombineerde HST (oestrogenen en progesterony positieve effecten heeft op een gevoel wan welzijn op de lange termijn. Echter, 
zowel controle proefpersonen $(n=11)$ als HST gebruiksters $(n=11)$ waren bij de baseline meting niet in klinische mate gestoord op werschillende aspecten van welzijn (slaspkwaliteit; holuamelijke en psychische klachten etc.). Positieve effeeten van HST op geheugenfuncties konden alleen worden aangetoond als gebruiksters werden vergeleken met bun eigen baselinefunctionerton. Uit deze studie bleek dat progestagenen niet de positieve effecten van oestrogenen op welzijn of geheugenfuncties tegenwerken. De resultaten gaven ook aan dat het design bepalend kan zijn voor het vinden van effecten. Namelijk, bij experimenten waarbij een cross-over design werd gebruikt waarin proefpersonen worden vergeleken met zichzelf, zonder en met HST, worden vank wel effecten werden gevonden op geheugenfuncties. Onderzoeken die een parallele groepen design gebruikten, waarbij de prestaties van twee groepen (HST gebruiksters versus niet gebruiksters) worden vergeleken, vonden vaker geen effecten wan HST op geheugenfuncties.

Er moet echter in aanmerking worden genomen dat er niet dubbel-blind getest kan worden bij HST (ontrekkings-bloeding en lof vermindering opvliegers) en dat verwachtingseffecten ten aanzien van gebruik de resultaten kunnen beinvloeden.

Hiermee in overeenstemming zijn de bevindingen wan een case-controle studie beschreven in hoofastuk 11. In dit onderzoek waren proefpersonen (vrouwen van middelbare leeftijd, afkomstig. uit het MAastricht Aging bestand) zich niet bewust van het doel wan het onderzoek. In dit onderzoek werden van HST geen positieve effecten gevonden op de mate van het ervaren welbevinden of de geheugenklachten. Hieruit kunnen we afleiden dat de bevindingen van hoofdstuk 12 ten aanzien van subjectief welbevinden mogelijk inderdaad door verwachtingen ten aanzien van HST gebruik kunnen zijn beïnvloed.

Uit de case-controle studie beschreven in hoofdstuk $I /$ bleek dat HST gebruiksters (n=23) wel gemiddeld sneller waren op basale informatieverwerkings-taken als vergeleken met controles $(\mathrm{n}=319)$. De bevinding dat de vrouwen in hoofdstuk 11 minder subjectief welbevinden raporteerden, maar wel een snellere informatieverwerking lieten zien vergeleken met controle proefpersonen, geeft verder aan dat basale sensori-motorische snelheid onafhankelijk van subjectief ervaren welbevinden kan zijn verbetererd. Dat er in deze studie geen effecten wan HST werden gevonden op geheugenfuncties kan mogelijk worden toegeschreven aan het gebruik van verschillende designs (zie hst 10). Anderzijds kan het liggen aan de verschillen tussen de geheugentaken die zijn gebruikt. Mogelijk was onze taak te moeilijk om de algemeen activerende effecten van HST atan het licht te laten komen.

\section{Conclusites}

Concluderend kunnen we stellen dat leeftijdsgerelateerde cognitieve achteruitgang blijkt uit een vertraging van cognitieve functies, wat met name duidelijk wordi bij complexe taken. Verder wordt een deel van de leeftijdsgerelateerde variantie verklaard door cognitieve traagheid en subjectief ervaren gezondheid. Met betrekking tot de modellen voor leefijdsgerelateerde cognitieve achteruitgang, hebben scopolamine en milde hypoxie hun predictieve waardle bewezen. Hyperventilatie was geen beter model dan hypoxie, maar fysieke uitputting kan wel worden gebruikt als model om cognitie-verbeterende stoffen te testen. Andere modellen, of combinatie modellen, moeten eerst verder worden onderzocht met betrekking tot hun braikbaarheid.

Cafeine liet positieve effecten zien op geheugenfuncties bij mensen van middelbare leeftijd terwiji dezelfde dosering negatieve effecten had bij jongeren. Cafeïne had negatieve effecten in het hypoxie model en positieve effecten na fysieke inspanning Dit leidde tot de hypothese dat de subjectieve interpretatie van verhoogde arousal door cafeine consumptie belangrijk kan zijn voor 
het effect dat cafeine heeft op de cognitieve functies. Een allernatieve hypothese is dat cafeme cognitieve functies kan beïnloeden wia verschillende nearotransmitter systemen, een effect dat het meest duidelijk is als er sprake is yan een functionelle, mat geen structurele, deficièntie. Experimenten natr het effect van cafeine hebben laten zien dat het gebruik van verschillende modellen nodig kan zijn om een mogelijke cognitie-verbeterende stof te testen. Het gebruik vam verschillende modellen zal toekomstige onderzoekers mogelijk maken om de effectiewe dosis, de mogelijke wijze watrop een middel aangrijpt en de testen die moeten worden gebruikt vast te stellen, om zo het effect van een middel te kunnen evalueren bij de doelgroep.

Bovendien laten de resultaten van het HST onderzoek zien dat een benadering die gericht is op instandhouding en bescherming wan fysiologische systemen vuchtbaar kan zijn in de zoektocht nat cognitie-verbeterende stoffen voor leeftijdsgerelateerde cognitieve achteruitgang Dit proefschrift heeft aangetoond dat er verschillende methoden zijn om cognitie-verbeterende stoffen te testen. Modellen van leeftijdsgerelateerde cognitieve achteruitgang (scopolamine, milde hypoxie, fysieke uitputting) kunnen worden gebruikt om cognitie-verbeterende stoffen te testen. Verder kan het effect van een middel in een aantal gevallen via cross-sectioneel onderzoek worden onderzocht. 


\section{Dankwoord}

Dat een niet gering aantal AIO's ermee stopt na een jaar en dat de rest in meer of mindere mate licht psychiatrisch gestoord geraakt in de jaren daarop volgend, geeft aan dat het schrijven var. een proefschrift niet gemakkeljk is. Je doet het dan ook niet alleen en er zijn veel mensen die ik wil bedanken en zonder wie dit proefschrift niet tot stand was gekomen.

Hooggeleerde Jolles, hooggeachte promotor, beste Jelle. Allereerst bedankt voor de kanis die je me hebt gegeven om aan deze klus te beginnen. Als we een afspraak hadden, wist je tre steeds te enthousiasmeren. Je multidisciplinaire aanpak is leerzaam. Ik hoop dat we het komend jaar samen nog meer interessante studies opzetten en beschrijven.

Zeergeleerde Riedel, geachte co-promotor, beste Wim, bedankt. Ik heb van jou meer geleerd dan in een opzicht. Je hebt steeds tijd wrij gemaakt voor me en dat heb ik zeer gewaardeerd. Hoewel niet altijd even makkelijk, ben ik door onze discussies meer toegespitst en strenger geworden, iets dat de stukken zeker ten goede is gekomen.

Leden van de leescommissie, ik wil jullie bedanken voor de tijd die jullie hebben genomen om het manuscript te lezen. Met name wil ik noemen prof. dr. Harald Merckelbach. Hooggeleerde Harald, ik ben erg blij dat je mijn stukken na 4 jaar opnieuw will beoordelen. Dr. Keith Wesnes, I enjoyed England very much. Thank you for joining this party.

Dr. Asker Jeukendrup en de BW studenten, bedankt woor het mogen deelnemen aan jullie duurstudie met de atleten. Dr. van der Steegen van de Sara Lee group wil ik bedanken voor de financiële steun en de inhoudelijke opmerkingen bij de cafeïne artikelen. Dr. Ries Simons van het Lucht- en Ruimtevaart Instituut te Soesterberg, bedankt voor de onderhoudende methodologische discussies over hypoxie en voor het lenen van de maskers. Sabine Kroeze, dank je voor het mogen de uitleg en het lenen wan de capnograaf. Prof.dr. Marcel van den Hout, ik vond het leuk dat je het hypoxie stuk door wilde lezen en voorzien van commentaar: ik vond onze discussie en met name je houding daarbij erg inspirerend. Dr. Fred Brouns en Ir. Eva Kovacs van het Sandoz Nutrition Lab, ik heb genoten van de efficiënte manier waarop we samen het cafeine-atleten onderzoek hebben gedaan. Christine Willekes, mijn dank is groot voor het mogen participeren in je hormonen follow-up studie en de prettige en gezellige samenwerking. Prof. Dr. Hubert Schouten, ik kan je niet genoeg bedanken voor alle keren dat ik weer langs mocht komen met allerlei statische vragen die je altijd weer geduldig beantwoordde. Ir. Nico Roozendaal, ook jij was altijd weer even aardig om me to helpen met data invoer en omzetting en zonder jou was het me niet gelukt de MAAS data te tacklen. Hooggeleerde Charlie Kaplan, I consider you to be my friend and I am very happy you spend so much of your time to work with me on the estrogen data. I hope that we can do more studies together in the future. Zeergeleerde Peter Houx en Martin van Boxtel, dank jullie voor de prettige samenwerking bij de hoofdstukken $3,4,5$ en 11 .

Dan zijn er altijd hopen stagiaires zonder wie de meeste studies nooil zouden worden uitgevoerd. Ik wil op eerste plaats bedanken de geleerde docterandus Jeroen Schmit, paranimf. Niet alleen was je zo goed dat ik je niet eens kon betrappen op fouten in je scriptie, zonder jou zou dit boekje niet zo mooi zijn gelayout dank zij de wele vrije uren die je gewillig doorbracht achter de computer. Ik ben blij dat we nu eindelijk op dezelfde kamer zitten en kunnen werken 
terwijl we roken. Je wint de nobelprijs hoe dan ook nog wel, je hebt immers ook de etage in de binnenstad gekregen. Geleerde Patricia Hameleers, dank je voor de fun in het lab bij de hypoxie experimenten, ik vind het tof dat jij je nu eindelijk op de cafeine data hebt gestort. Geleerde dokter Marjolein Boshuisen, niet alleen was jij erg goed en efficient met het helpen verzamelen van literaturur voor hoofdstuk 10 , je bent een fijne en lieve vriendin, ik hoop dat we samen nog valak kunnen pentjakken. Verder wil ik bedanken voor de hulp bij de experimenten Stella Seij en Isabelle van de Veen en natuurlijk alle proefpersonen die zich vrijwillig onderwierpen aan mijn experimenten.

Een groot deel van je tijd breng je door op je werk, met name wil ik bedanken Carlein Karimoen, jij hebt me als schaduwnimf en vriendin geweldig geholpen en ik ben blij dat ik jou en Frans heb mogen leren kennen. Verder wil ik iedereen van de vakgroep bedanken die het gezelliger en daarmee een stukje dragelijker hebben gemaakt in moeilijke tijden. Daarbuiten wil ik de mensen van de pentjak club bedanken voor het kanaliseren van de frustratie, net als Joghi en zijn baasjes zonder wie ik deze periode een stuk gestresster had doorgemaakt.

Het is raar om je vrienden als laatste te noemen, want die zijn eigenlijk het belangrijkste in een AIO periode. Op de eerste plaats, geleerde Mirjam Kampman, lieve paranimf. Je hebt me de hele periode gesteund op alle fronten en ik ben blij dat ik nu al bijna 10 jaar jou heb mogen kennen. Roger en Marjan, zonder jullie coaching was ik er allang mee op gehouden! Verder wil ik bedanken: het $\mathrm{D} \& \mathrm{D}$ clubje, en alle andere vrienden en familie, vooral mijn lieve moeder, voor het plezier, de nooit aflatende steun en hun vertrouwen in de goede afloop.

Remco, het is een objectief gegeven dat jij ooit bent gekozen alls beste, meest begeerde, grappigste, en liefste man door een onafhankelijk panel van dames. Je bent dan ook het fundament dat dit proefschrift draagt. 


\section{Curriculum vitae}

Eef Hogervorst werd geboren in Heerlen op 16 juli 1968 . Op 14-jarige leeftijd ontdelkte zij in Mexico de wereld van het brein in een Scientific American en besloot om onderzoeker van hersenen en gedrag relaties te worden. Van 1984 af had Eef verschillende baantjes (paatrdrijles en bijles Engels, markt, huis-aan huis-werk, data-invoer voor de kamer van Koophandel en de Thuiszorg voor ouderen in Amsterdam) en in 1987 behaalde zij haar atheneum-diploma aan de Dag en Avond-school te Amsterdam. In datzelfde jaar begon zij in Maastricht met de studie Geestelijke Gezondheidskunde. In het tweede en derde jaar van haar studie participeerde Eef in onderzoek met R. Kreutzkamp en J. Jansen naar de effecten van eetstoornissen en stemming op body-image. In het derde jaar werd zij aangenomen als student-assistente bij het Institut voor Veiligheid, Geneesmiddelen en Verkeer. Hier begon zij een literatuur-studie naar de effecten van oestrogenen op cognitieve functies. Om zich meer te verdiepen in neuropsychologie, volgde Eef een stage bij het Psychomedisch Centrum Vijverdal en keuzeblokken bij de universiteit van Nijmegen. Aldus cognitief gesterkt kreeg Eef in het vierde jaar van haar studie een baan als test-assistente bij het Behandelteam Angsstoornissen van de RIAGG Maastricht. Geprikkeld door de resultaten van gedragstherapie besloot ze cen jaar parttime een stage als therapeute te volgen. Haar hart lag echter toch bij het experimentele onderzoek en in 1993 startte zij samen met Harald Merckelbach een reeks experimenten naar de effecten van EMD-R, een nieuwe therapie voon trauma's.

Ongeveer 10 jaar na dato was Eef gelukkig te kunnen starten als AIO bij de vakgroep Psychiatrie en Neuropsychologie waar zij eindelijk Hersenen en Gedrag onderzoek mocht doen. Na haar promotie zal zij een aanstelling krijgen waarbij onderwijs en onderzoek naar Hormonen en Cognitieve ontwikkeling de invulling bepalen.

Naast haar werk zijn haar hobbies tussen de bedrijven door: tekenen, paardrijclen, pencak silat, duiken, deltavliegen, lezen, wandelen en gezellige dingen met vrienden ondernemen. 


\section{Publicaties}

\section{W Wetenschappelijke publicaties in internationalle tijdschriften}

Hogervorst E. Riedel, W.J, Schmit, J.A.J., \& Jolles, J. (Accepted Dec 1997). Caffeine improves memory performance during distraction in middle-aged, but not in young or old subjects. Human Psychophatmacology.

Hogervorst, E., Riedel, W.J., Jeukendrup. A., \& Jolles, J. (1996) Lack of cognitive deficiencies due to strenuous excercise. Perceptual and Motor Skills, 83 (2), 479-488.

Riedel, W. J.4 Hogervorst, E., Leboux, R.L.A.M., Verhey, F.J., Van Praag, H.M., Jolles, J. (1995). Caffeine attenuates scopolamine-induced memory impairement in humans. Psychopharmacology, $122(2), 158-168$.

Merckelbach, H., Hogervorst, E., Kampman, M., \& De Jongh, A. (1994). Effects of 'eyemovement desensitisation' on emotional processing in normal subjects. Behavioral and Cognitive Psychotherapy, 22 (4), 331-335.

\subsection{Annotaties / Abstracts}

Hogervorst, E., Riedel, W.J., Schmitt, J.A.J., Houx, P.J., \& Jolles, J. The effect of age, health, and speed of information processing on complex memory performance. Nieuwsbrief NVP, jaargang 12, nummer 3 (Supplementen), 1997.

Hogervorst, E., Riedel, W.J., van Boxtel, M.P.J., Visser, P.J., Hameleers, P., Seij S., Jolles, J. Hypoxia and hyperventilation as models for cognitive defiencies. European Neuropsychopharmacology 6(S3) June: 145,1996

Riedel. W., Hogervorst, E., Verhey, F., Jolles, J. Scopolamine is a model of memory dysfunction not of aging. European Neuropsychopharmacology. 6(S3) June: 144, 1996

Hogervorst, E., Riedel, W.J., van Boxtel, M.P.J., Jolles, J. Hypoxia and hyperventilation as models for cognitive defiencies. European Neuropsychopharmacology. 6(S4) Sept: 166, 1996

Riedel, W.J., Hogervorst, E., \& Jolles, J. (1995). Cognitive enhancers and human aging. European Neuroscience Annual Meeting. Amsterdam, the Netherlands, 3-7 september.

Riedel,W., Leboux, R.L.M., Hogervorst,E., Quist, A., van der Vusse, A., Verhey, F.R.J., \& Jolles, J. (1994). Caffeine enhances memory function after cholinergic blockade. Neurobiology of Aging, 15 (1), S100-101.

\subsection{Wetenschappelijke publicaties in nationale tijidschriften}

Merckelbach, H., Hogervorst, E., Kampman, M., \& De Jongh, A. (1994). 'Eye-movement desensitisation' heeft geen effect op de emotionele reactiviteit van "nomale' proefpersonen. Gedragstherapie, vol. $27(1), 33-49$

Merckelbach, H., Hogervorst, E., \& Kampman, M. (1993). Geen ondermijnend effect van 'Eyemovement desensitisation" op het visueel geheugen. Directieve Therapy, jaargang 13, nowember. Bohn Stafleu Van Loghum.

\subsection{Boekbijdragen}

Riedel, W.J., Hogervorst, E., Verhey, F.R.J., \& Jolles, J. (1997). Measuring Cognitive Abilities in Cholinergic Dysfunction. In: I. Hindmarch P.D. Stonier (Eds.) Human Psychopharmacology: Measures and Methods; Vol VI. Chichester: Willey, pp. 29-56. 
Hogervorst, E., Riedel, WJ., Rozendaal, N., van Boxtel, M.P.J., \& Jolles, J. (1995). Smokingand cognitive complaints. In: J. Jolles, P.J. Houx, M.P.J. van Boxtel, \& R. W. H.M. Ponds (Eds.) The Maastricht Aging Study: Determinants of cognitive aging. Maastricht, the Netherlands: Neuropsych Publishers, $105-110$.

Riedel, W.J, Hogervorst, E., \& Jolles, J. (1995). Cognition enhancers and aging. In: J. Jolles, P.J. Houx, M.P.J. van Boxtel, \& R.W.H.M. Ponds (Eds.). The Maastricht Aging Study: Determinants of cognitive aging. Maastricht, the Netherlands: Neuropsych Publishers, 149 . 156.

Riedel, W.J., Leboux, R.L.A.M., Hogervorst, E., Quist, A., Vusse, A. van de., Verhey, F.R.J. \& Jolles, J. (1994). Caffeine and Nicotine selectively enhance Memory and Attention after Cholinergic Blockade. In W. wan Tilburg (Ed.), The Borderland Between Normal and Pathological Aging. Amsterdam, The Netherlands: International Psychogeriatric Association, p.47.

\subsection{Rapporten/proceedings}

Hogervorst, E., Riedel, W.I., Kovacs, E., Brouns, F., \& Jolles, J. The effect of caffeine and strenuous exercise on cognitive performance. Report for Sandoz Nutrition. December, 1996

Hogervorst, E., Riedel, W.J., van Boxtel, M.P.J., \& Jolles, J. Estrogen Replacement Therapy and cognitive functioning in healthy, elderly women. Interim Report for Organon. June, 1996

\subsection{Congrespapers}

Hogervorst, E. (1995). Modellen voor cognitieve dysfuncties. Symposiumbundel VBGGK "Onderzoek onder de loep"- Maastricht, 9 feb

Hogervorst, E. (1995). Destrogenen en postmenopausale vrouwen. Symposiumbundel "Een Vrolijke Oude Dag" 1-2 april Amsterdam.

Hogervorst, E. (1995). De behandeling van ouderdomsgerelateerde klachten. Symposiumbundel "Een Vrolijke Oude Dag" 1-2 april Amsterdam.

Hogervorst, E. (1995). Mentaal functioneren en veroudering. Symposiumbundel "Een Vrolijke Oude Dag" 1-2 april Amsterdam.

Hogervorst, E., \& Riedel, W.J. (1994). Models of cognitive aging. European Summer School on Cognitive Neuroscience june 24-july.

\subsection{Voordrachten en posterpresentaties}

Hogervorst, E. The effect of age, health, and speed of information processing on complex memory performance. Paper presented at the 6e Wintercongres Nederlandse Vereniging voor Psychonomie, Egmond aan Zee, December 19-20, 1997.

Hogervorst, E. 'Models of cognitive dysfunction'. Presentatie in kader van de WetenschapsdagInstitute Brain and Behavior, 26 September, 1997

Hogervorst, E., Riedel, W.J., wan Boxtel, M.P.I., Jolles, J. Hypoxia and hyperventilation as models for cognitive defiencies. Posterpresentation at the 9th Congress of the European College of Neuropharmacology, Amsterdam, September 21-25, 1996.

Hogervorst, E., Riedel, W.J., van Boxtel, M.P.J., Visser, P-J, Hameleers, P., \& Seij S. Hypoxia and hyperventilation as models for cognitive defiencies. Oral presentation 15 min at the $x \times$ th Collegium Internationale NeuroPsychopharmacologicum Congress, Melbourne, Australia, June 23-27, 1996. 
Hogervorst, E., Riedel, W.J., van Boxtel, M.P.S. \& Jolles, J. Hypoxia and hyperventilation as models for cognitive defiencies. Oral presentation $20 \mathrm{~min}$ at the 1996 Annual Meeting of the Psychobiology Section of the British Psychology Society. The Old Dungeon Ghyll Hotel, Cumbria, England, September 18-20 1996

Hogervorst, E, Riedel, W, \& Jolles, J.. Models of cognitive aging. Oral presentation 20 min at the Symposium on Brain, Cognition, and Behavior, Maastricht, November 29th, 1996

Hogervorst, E. 'Modellen voor cogninieve dysfuncties'. Symposium VBGGK 'Onderzoek onder de loep* Maastricht, 9 Februari, 1995.

Hogervorst, $\mathrm{E}$. Visuele Illusies". Voordracht en demonstratie in kader van open dag faculteit Psychologie, 4 March 1995 and 21 October, 1995

Hogervorst, E. 'Cognitive modellen van veroudering'. Presentatie in kader van de Wetenschapsdag vakgroep, 3 Februari, 1995

Hogervorst, E. 'Cognitieve modellen van veroudering'. Presentatie in kader van NWO werkgemeenschap' Gerontologie en geriatrie* Driebergen, 6-7 June, 1994.

Hogervorst, E. 'Experimenteel-psychologisch onderzoek naar 'Eye-movement desensitisation and reprocessing. Presentatie in kader VGT Najaarsconferentie Dalfsen $4-5$ November, 1993. 